\title{
Mitogenic and Metabolic signals regulate mTOR complex 1 function to control GSK3 $\beta$ nuclear program
}

By

Stephen James Bautista

B.Sc., Ryerson University, 2014

\author{
A dissertation \\ presented at Ryerson University \\ in partial fulfillment of the requirements for the degree of \\ Doctor of Philosophy \\ in the program of Molecular Science
}

Toronto, Ontario, Canada, 2019

C) Stephen James Bautista, 2019 


\section{AUTHORS'S DECLARATION FOR ELECTRONIC SUBMISSION OF A DISSERTATION}

I hereby declare that I am the sole author of this dissertation. This is a true copy of the dissertation, including any required final revisions, as accepted by my examiners.

I authorize Ryerson University to lend this dissertation to other institutions or individuals for the purpose of scholarly research

I further authorize Ryerson University to reproduce this dissertation by photocopying or by other means, in total or in part, at the request of other institutions or individuals for the purpose of scholarly research.

I understand that my dissertation may be made electronically available to the public. 
Mitogenic and Metabolic signals regulate mTOR complex 1 function to control GSK3 $\beta$ nuclear program

Stephen James Bautista

Doctor of Philosophy, 2019

Molecular Science, Ryerson University

\begin{abstract}
Mitogenic and metabolic signalling are two cell pathways that control different aspects of cellular physiology including, growth, proliferation, metabolism, and transcription. Mitogenic signalling involves mitogens and growth factors to stimulate various receptor signalling pathways such as epidermal growth factor receptor (EGFR), while metabolic signalling involves proteins that sense changes in abundance of specific nutrients or metabolites such as amino acids and ATP. Here, I have uncovered that EGFR signalling is controlled by clathrin nanodomains at the plasma membrane, yet this requirement for clathrin does not reflect a role for receptor internalization in EGFR signalling. Specifically, I found that clathrin is required for activation of the key signaling intermediate Akt by EGFR upon EGF stimulation. Furthermore, I have also resolved a series of signals including Phospholipase $\mathrm{C} \gamma 1$ (PLC $\gamma 1$ ) that may control EGF stimulated Akt activation by modulating the assembly of clathrin into plasma membrane nanodomains. These findings suggest that clathrin nanodomains at the plasma membrane are important for controlling EGFR signalling, thus impacting mitogenic signaling.
\end{abstract}

A downstream signalling pathway controlled by Akt is the Glycogen synthase kinase 3 
(GSK3) pathway. GSK3 phosphorylates and thereby regulates a wide range of protein substrates involved in diverse cellular functions. Some GSK3 substrates, such as c-Myc and Snail, are nuclear transcription factors, suggesting the possibility that GSK3 function is controlled through regulation of its nuclear localization. I found that perturbations in mTOR complex 1 (mTORC1) leads to partial redistribution of GSK3 from the cytosol to the nucleus and to a GSK3 dependent reduction of the levels of both c-Myc and Snail. In addition to conditional nuclear localization, GSK3 was also detected on several distinct endomembrane compartments, including lysosomes. Consistently, disruption of various aspects of the function and regulation late endosomes/lysosomes resulted in perturbation of GSK3 nucleocytoplasmic shuttling and activity. Furthermore, I found that DEPDC5, a subunit of the lysosomal amino-acid sensing GATOR1 complex, controls amino acid sensing mechanisms to regulate GSK3 nucleocytoplasmic shuttling. These findings uncover a new signalling axis that is controlled by specific aspects of both mitogenic and metabolic signalling, which may interface with the nucleus to reprogram transcriptional cellular networks for growth and proliferation. Understanding how mTORC1GSK3 signalling impacts transcriptional networks may be an important target for different therapies and treatments against diverse forms of cancer. 


\section{Acknowledgements}

I would like to thank my supervisor Dr. Costin N. Antonescu for all the amazing opportunities given to me, since undergrad, masters, and transition into $\mathrm{PhD}$. Everything from mentorship, guidance, career advice, mental break downs, successes, sports talks, thank you for it all. Without you, all things would not be possible for me in this $\mathrm{PhD}$ career.

I would also like to thank my PhD committee members, Dr. Roberto Botelho and Dr. Raffi Karshafian, for guiding and advising me throughout my PhD career. Everything you both said and did, help mold me into the Doctor of Philosophy I am today, which I am truly grateful for.

To Scixchange (Dr. Emily Agard and Leigh Paulseth) and Let's Talk Science (Nicole Kaiser), for teaching the ways of science communication and giving me the opportunity to be an outreach site coordinator. Science communication taught me how to communicate my bench research and all the great things science has to offer to the general public, making it both fun and interesting. Thus, I intend to take science communication with me into my future career and whereever the science world may take me.

To the 2019 NBA champions of the world: Toronto Raptors, for giving me study and writing breaks (mostly stress over many games) but also motivation, that anything is truly possible if you believe and focus on your goal. \#WeTheNorth \#WeTheChamps \#LetsGoRaptors 
To my Antonescu and Botelho lab sisters, Dr. Farnaz Fekri, Dr. Stefanie Lucarelli, and Dr. Victoria Hipolito. We went through 4 years of undergraduate and 4.5 years of graduate school together. Now look at us, all Doctors, we went through hell and back countless times, and it brought us to this point. Guys we did it! I love you all.

To my best friends, Brendon Fresco, Kenneth Villasenor, and Francis Caracas, for nights that I really needed after failures, bumps on the road, and even successes during my $\mathrm{PhD}$ career. To my extended family who always supported me and attempted understand how my $\mathrm{PhD}$ works and the science that I am doing.

Finally, last but not the least, Mom, Dad, and my partner, Tiffany, for all the love and support given during my $\mathrm{PhD}$ career. Everything you have offered or given has help in some way shape or form, shaping the man and Doctor I am today, love you all. 


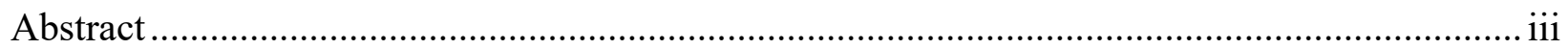

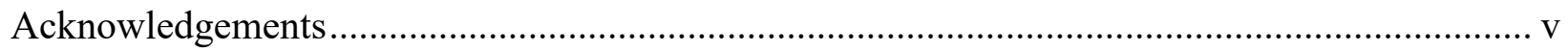

List of Appendices ........................................................................................................

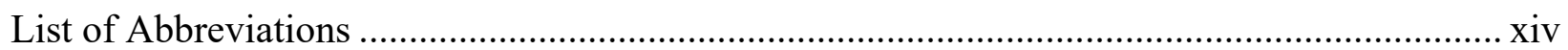

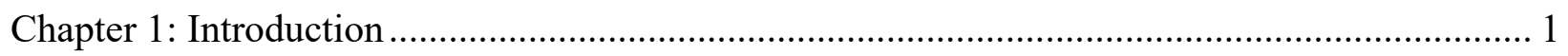

1.1.1 The adaptability of human physiology .................................................................. 2

1.1.2 Sensing signals from other cells: Receptors as sensors of intercellular communication... 5

1.1.3 Internal sensing: Intracellular sensor in metabolism ............................................. 10

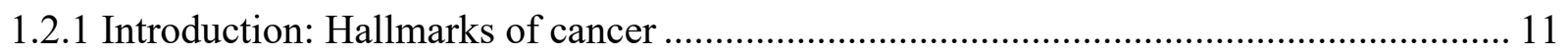

1.2.2 Emerging concepts for Hallmarks of cancer: metabolism and Warburg effect.............. 16

1.2.3 Cancer metabolism: Glycolysis-associated pathways fuel biomass production............. 18

1.2.4 Cancer metabolism: Oxidative phosphorylation and therapeutic resistance ................. 21

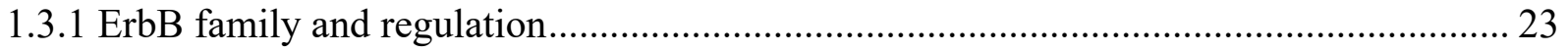

1.3.2 EGFR activation of Akt-mTORC1 pathway....................................................... 24

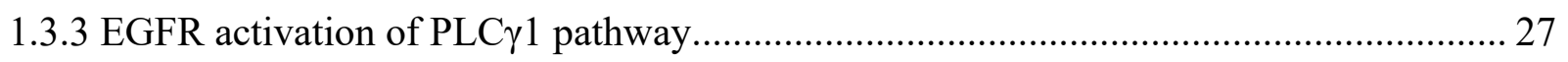

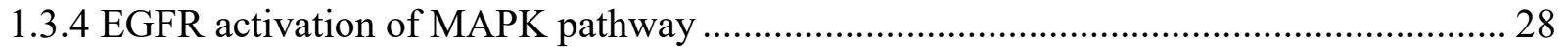

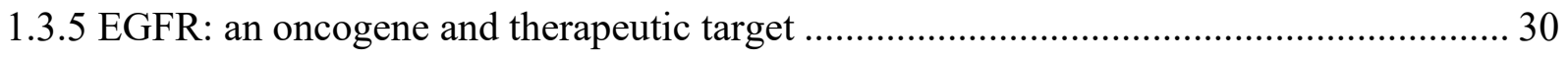

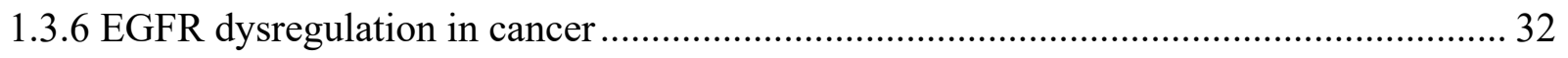

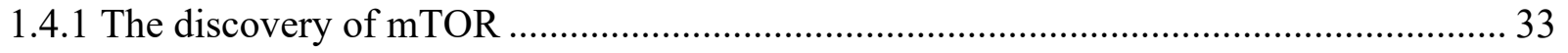

1.4.2 mTOR: two different complexes mTORC1 and mTORC2 ….................................. 34

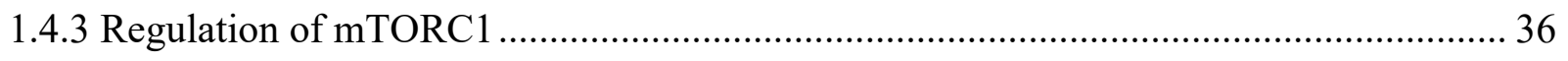

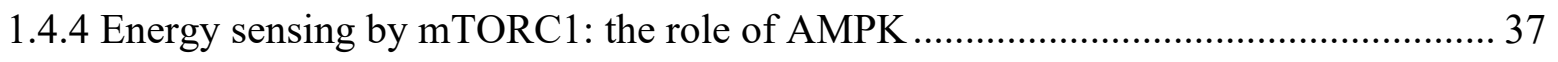

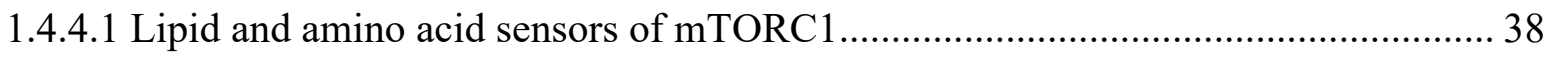

1.4.4.2 Regulation of mTORC1: signalling output ................................................. 40

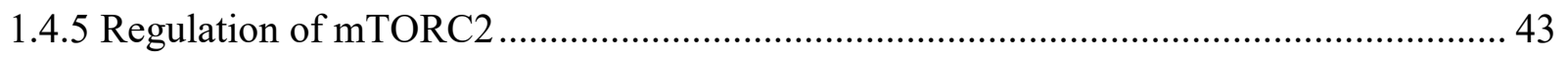

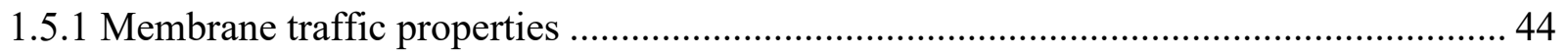

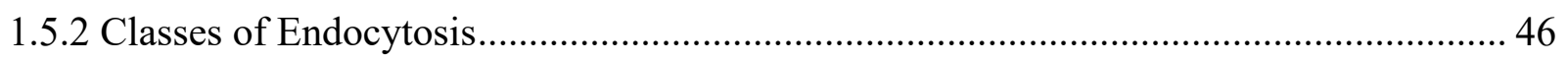

1.5.3 Endocytic membrane traffic after internalization .............................................. 49

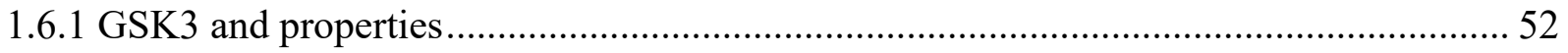




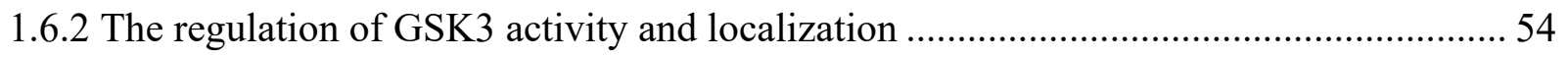

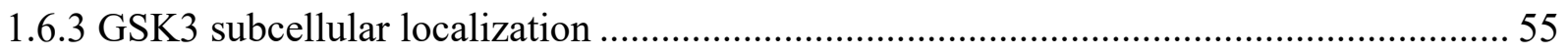

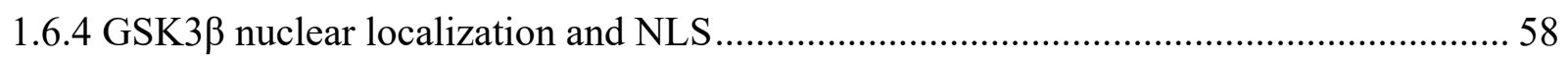

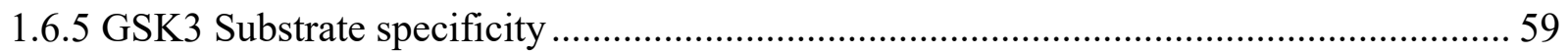

1.6.6 GSK3 in disease: Diabetes, Alzheimer's disease, and Cancer ......................................... 60

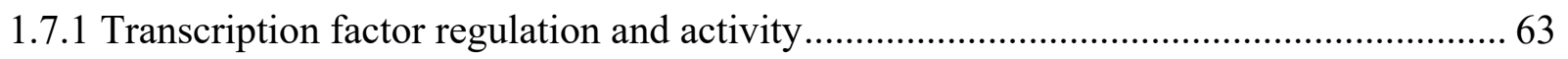

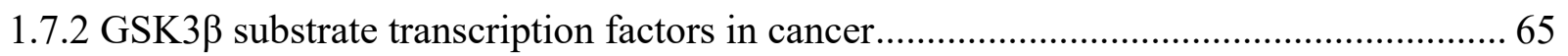

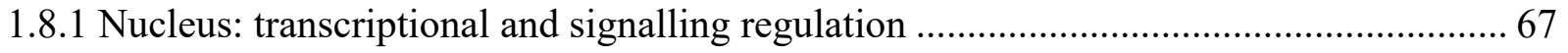

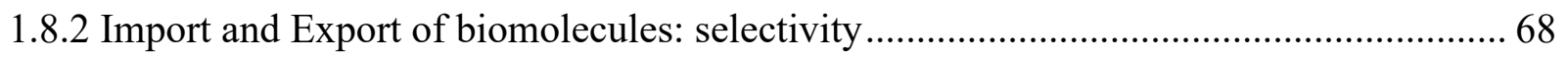

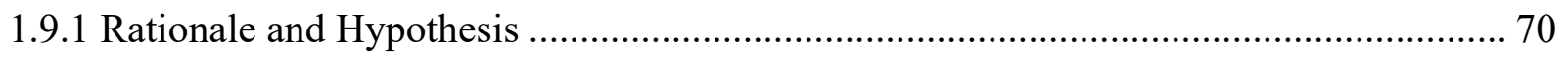

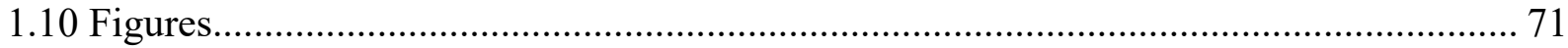

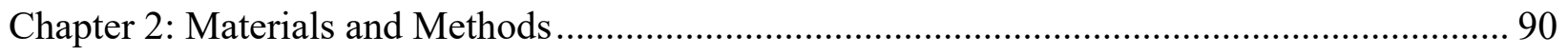

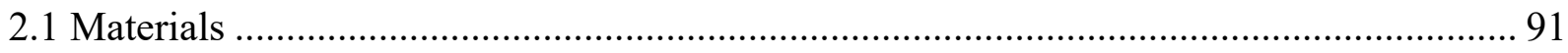

2.2 Cell lines, cell culture and inhibitor treatment............................................................. 92

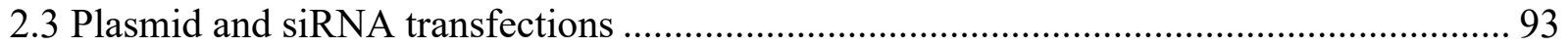

2.4 Whole -cell lysates, subcellular fractionation and Western blotting .................................. 94

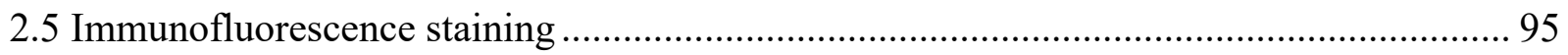

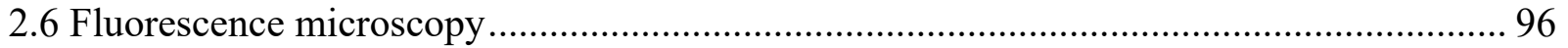

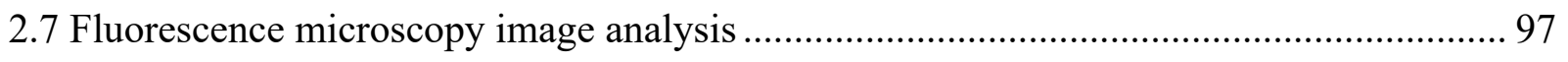

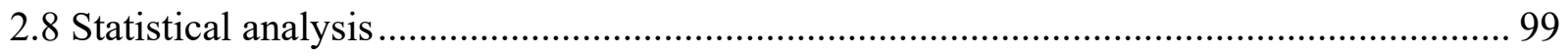

Chapter 3: Clathrin-dependent control of mitogenic signalling by EGFR ………….................. 100

3.1 Perturbation of clathrin, but not that of dynamin2, impaired Akt activation after EGF

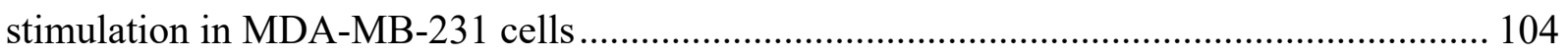

3.2 PLC $\gamma 1$ affects CME dynamics and Akt phosphorylation but not Erk phosphorylation... 106

3.3 Dynamin1 perturbations affects EGFR and Akt phosphorylation but Erk activity .......... 107

Chapter 4: GSK3 localization and activity ........................................................................... 113

4.1 mTORC1 controls GSK3 $\beta$ nuclear localization and c-Myc levels .................................... 116

4.2 Metabolic cues regulate GSK3 $\beta$ nuclear localization via mTORC1 …......................... 119

4.3 Control of GSK3 $\beta$ nuclear localization does not require GSK3 $\beta$ S9 phosphorylation .... 122

Chapter 5: Lysosomes as signalling platforms for control of GSK3 nuclear translocation........ 133 
5.1 GSK3 3 is localized to several distinct membrane compartments within the cytoplasm, including the lysosome

5.2 Control of GSK3 $\beta$ nuclear localization and c-Myc levels requires Rab7-mediated

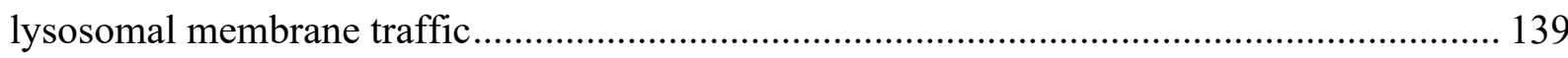

5.3 Specific amino acids acutely promote GSK3 nuclear export ………………………........ 141

5.4 DEPDC5 controls lysosomal localization of GSK3 $\beta$ and mTORC1-dependent

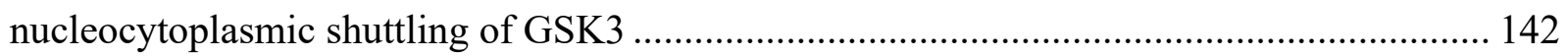

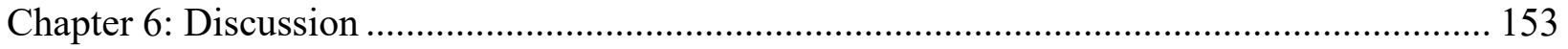

6.1 Chapter 3: Clathrin plasma membrane nanodomains are signalling platforms for EGF

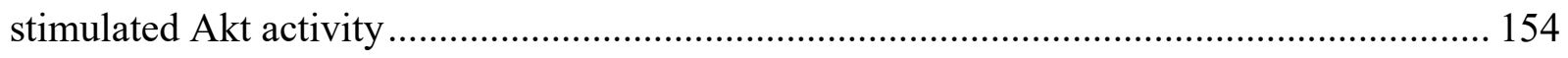

6.2 Chapter 3: Isoform specific regulation by Dynamin1 controls EGF stimulate Akt

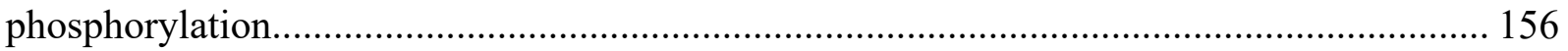

6.3 Chapter 3: PLC $\gamma 1$ modulates CME dynamics, in turn regulating EGF stimulated Akt

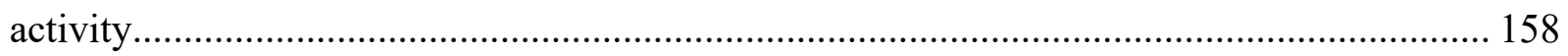

6.4 Chapter 4: Mechanism of control of GSK3 $\beta$ nuclear localization by mTORC1 .............. 159

6.5 Chapter 4: Regulation of GSK3 $\beta$ nuclear functions by mTORC1 ................................. 163

6.6 Chapter 5: Localization of GSK3 $\beta$ to multiple membrane compartments within the

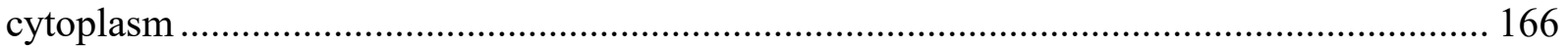

6.7 Chapter 5: Amino acid sensing of GATOR1 subunit DEPDC5 controls GSK3 $\beta$ nuclear

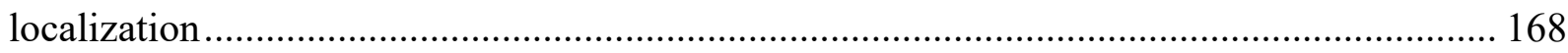

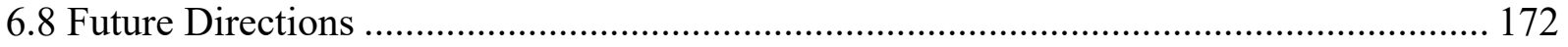

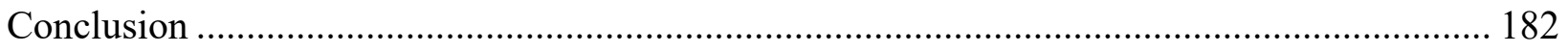

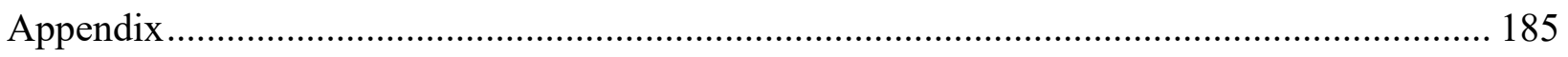

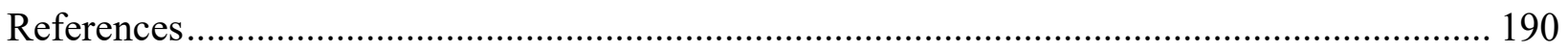




\section{List of Figures}

\section{Chapter 1}

Figure 1. 1 Cells sense environmental cues to alternate its cell physiology ............................. 72

Figure 1. 2 Ligand binding leads to receptor activation .................................................... 73

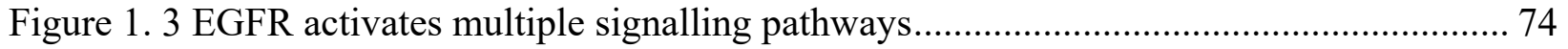

Figure 1. 4 Glycolysis and intermediates used in separate pathways .................................... 75

Figure 1.5 EGFR regulates the PI3K and Akt signalling pathway..................................... 76

Figure 1. 6 EGFR regulates the PLC $\gamma 1$ signalling pathway............................................... 77

Figure 1. 7 EGFR regulates the MAPK signalling pathway............................................... 78

Figure 1. 8 mTORC1 and mTORC2: complex differences ............................................. 79

Figure 1. 9 mTORC1 is regulated by both mitogenic and metabolic signalling. ...................... 80

Figure 1. 10 AMPK regulates mTORC1 by sensing energy changes.................................... 81

Figure 1. $11 \mathrm{mTORC1}$ amino acid sensing abilities regulated by multiple factors .................... 82

Figure 1. 12 mTORC1 activation leads to changes in translation and metabolism................... 83

Figure 1. 13 mTORC2 activation leads to changes in cell survival and cytoskeleton remodeling.

Figure 1. 14 Membrane traffic involves dynamic movement of material from different membrane

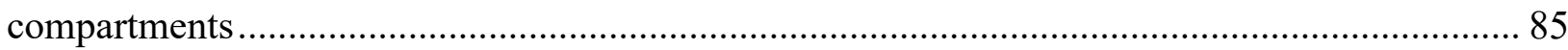

Figure 1. 15 Multiple endocytosis classes involve different mechanisms of internalization for

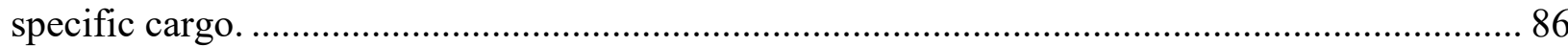

Figure 1. 16 GSK3 phosphorylation via different substrates................................................ 87

Figure 1. 17 GSK3 is localized to many membrane compartments. ..................................... 88

Figure 1.18 Dynamic nuclear import and export mediated by Ran GTPase ........................... 89

\section{Chapter 3}

Figure 3.1 siRNA gene silencing of clathrin and not dynamin2 perturb Akt phosphorylation after EGF stimulation in ARPE-19 cells

Figure 3. 2 clathrin perturbations inhibit Akt phosphorylation after EGF stimulation in MDA-

MB-231 cells. 110 
Figure 3. 3 PLC $\gamma 1$ perturbations affect CME dynamics inhibiting EGF stimulated Akt phosphorylation.

Figure 3. 4 Dynamin1 knockdown impairs EGF stimulated EGFR and Akt phosphorylation .. 112

\section{Chapter 4}

Figure 4. 1 mTORC1 inhibition decreases C-Myc and Snail levels in a GSK3 $\beta$-dependent

manner.

Figure 4.1. 1 GSK3 $\beta$ siRNA silencing, and GSK3 3 nuclear localization and function in MDA-

MB-231 cells

Figure 4. 2 Inhibition of PI3K/Akt/mTORC1 signals promotes GSK3 $\beta$ nuclear localization... 126

Figure 4. 3 Rapamycin-induced GSK3 $\beta$ nuclear localization is Ran-dependent. ...

Figure 4. 4 mTORC1 integrates multiple signals to control GSK3 $\beta$ nuclear localization. ........ 128

Figure 4.4. 1 AMPK silencing does not impact GSK3 $\beta$ nuclear localization

Figure 4.4. 2 Inhibition of autophagy induction by ULK1 siRNA gene silencing does not impact

rapamycin-induced GSK3 $\beta$ nuclear localization or reduction in C-Myc levels 130

Figure 4. 5 GSK3 $\beta$ Ser-9 phosphorylation is not required for GSK3 $\beta$ nuclear localization induced by inhibition of PI3K-Akt-mTORC1 signals.

\section{Chapter 5}

Figure 5. 1 GSK3 $\beta$ exhibits partial localization to several distinct endomembrane compartments

Figure 5. 2 Contribution of Rab7 and APPL1 to GSK3 $\beta$ localization 147

Figure 5. 3 Rab7 controls GSK3 $\beta$ nuclear localization and GSK3 $\beta$-dependent C-Myc levels. . 149 Figure 5. 4 Amino acid starved cells replenished with leucine, arginine, and non-essential amino acids + EGF export GSK3 $\beta$ from the nucleus 150

Figure 5. 5 DEPDC5 KO displaces GSK3 $\beta$ from the lysosome and GSK3 $\beta$ nuclear localization program is insensitive to amino acid deprivation 152 


\section{Chapter 6}

Figure 6. 1 DEPDC5 protein structure and potential mutations/truncations to identify GSK $3 \alpha / \beta$

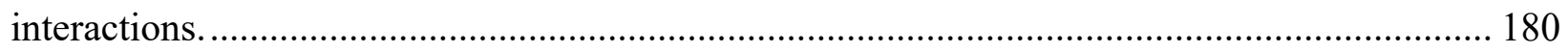

Figure 6. 2 CRISPR/Cas9 knockout of GSK3 $\beta$ in MDA-MB-231 breast cancer cells and rescue assay. 


\section{List of Appendices}

Figure A. 1 Clathrin perturbations does not impair EGFR autophosphorylation..................... 186 Figure A. 2 PLC $\gamma 1$ siRNA perturbs EGFR internalization but increases cell surface EGFR, while dynamin1 siRNA does not alter internalization but decreases cell surface EGFR.................. 187 Figure A. 3 Proximity biotinylation (BioID assay) shows interaction between DEPDC5 and

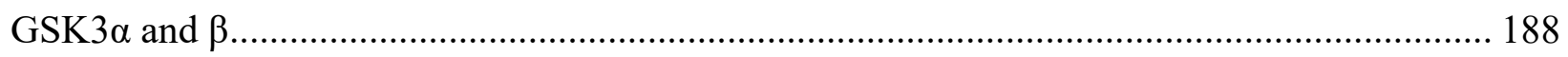

Figure A. 4 DEPDC5 KO hyperactivates mTORC1 activity. ............................................. 189 


\section{List of Abbreviations}

$\mathrm{AD}$

ADP

Akt

AMBRA

AMP

AMPK

AP2

APC

APP

APPL1

ATM

ATP

ATG

ATG16L

Axin

BACE1

Barkor

BCL-X

c-Myc

C/EBP

$\mathrm{Ca}^{2+}$
Alzheimer's disease

adenosine diphosphate

AKT serine/threonine kinase

autophagy and beclin 1 regulator 1

adenosine monophosphate

amp-activated protein kinase

adaptor protein 2

adenomatous polyposis coli

$\beta$-amyloid precursor protein

adaptor protein, phosphotyrosine interacting with $\mathrm{PH}$ domain and leucine zipper 1

ataxia-telangiectasia mutated

adenosine triphosphate

autophagy related protein

Atg like protein 16

axis inhibition protein

$\beta$-site APP-cleaving enzyme

Beclin-1 associated autophagy-related key regulator

B-cell lymphoma-extra large

C myc proto-oncogene, BHLH transcription factor

CCAAT-enhancer-binding protein

calcium 


\begin{tabular}{|c|c|}
\hline $\mathrm{CaM}$ & calmodulin \\
\hline Castor 1 & cytosolic arginine sensor for mTORC1 subunit 1 \\
\hline Castor 2 & cytosolic arginine sensor for mTORC1 subunit 2 \\
\hline CK1 & casein kinase 1 \\
\hline $\mathrm{Cbl}$ & Casitas B-lineage lymphoma \\
\hline ССР & clathrin-coated pits \\
\hline $\mathrm{CCV}$ & clathrin-coated vesicles \\
\hline CDK 4/6 & cyclin-dependent kinase $4 / 6$ \\
\hline $\mathrm{CHO}$ & Chinese hamster ovary \\
\hline CIE & clathrin independent endocytosis \\
\hline CME & clathrin mediated endocytosis \\
\hline Creb & cAMP response element-binding protein \\
\hline $\mathrm{CSC}$ & cancer stem cells \\
\hline CXCL12 & C-X-C motif chemokine ligand 12 \\
\hline CXCR4 & C-X-C chemokine receptor type 4 \\
\hline DAG & diacylglycerol \\
\hline DAPI & 4', 6-diamidino-2-phenylindole \\
\hline DEPDC5 & DEP domain containing 5 \\
\hline DEPTOR & DEP domain containing mTOR interacting protein \\
\hline DMSO & dimethyl sulfoxide \\
\hline DSH & dishevelled \\
\hline Gab1 & growth-factor receptor-bound protein 2-associated 1 \\
\hline EBSS & earle's balanced solution \\
\hline
\end{tabular}




$\begin{array}{ll}\text { EEA1 } & \text { early endosome antigen 1 } \\ \text { EGF } & \text { epidermal growth factor } \\ \text { eGFP } & \text { enhanced green fluorescent protein } \\ \text { EGFR } & \text { epidermal growth factor receptor } \\ \text { EIF4E } & \text { eukaryotic translation initiation factor 4E } \\ \text { EMT } & \text { epithelial-mesenchymal transition } \\ \text { ER } & \text { endoplasmic reticulum } \\ \text { ErbB2 } & \text { erb-B2 receptor tyrosine kinase 2 } \\ \text { ERK } & \text { extracellular signal-regulated kinase } \\ \text { F6P } & \text { fructose 6 phosphate } \\ \text { FGFR } & \text { fibroblast growth factor receptor } \\ \text { FIP200 } & \text { focal adhesion kinase family interacting protein of 200 kD } \\ \text { FKBP12 } & \text { 12-kDa FK506-binding protein } \\ \text { FLCN } & \text { folliculin } \\ \text { FNIP } & \text { folliculin-interacting protein } \\ \text { FoxO } & \text { forkhead box O } \\ \text { FRAT1 } & \text { frequently rearranged in advanced T-cell lymphomas 1 } \\ \text { FRB } & \text { FKBP12-rapamycin binding } \\ \text { Fyn } & \text { growth factor receptor protein 1 } \\ \text { G6P } & \text { Gaboto-oncogene, src family tyrosine kinase } 6 \\ \text { GAP } & \text { phosphate } \\ & \end{array}$

GATOR $1 / 2 \quad$ Gap activity towards Rag 1/2 complex 


\begin{tabular}{|c|c|}
\hline GBP & GSK3 binding protein \\
\hline GDP & guanosine diphosphate \\
\hline GEF & guanyl exchange factor \\
\hline GlcNAc-6-P & N-acetylglucosamine-6-phosphate \\
\hline GPCR & G-protein coupled receptor \\
\hline Grb2 & growth factor receptor-bound protein 2 \\
\hline GSK3 & glycogen synthase kinase 3 \\
\hline GTP & guanosine triphosphate \\
\hline HA & hemaglutinin \\
\hline HIF $1 \alpha$ & hypoxia inducible factor 1 subunit $\alpha$ \\
\hline IL & interleukin \\
\hline IP3 & inositol trisphosphate \\
\hline IRS & insulin receptor substrate \\
\hline KICKSTOR & $\underline{\mathrm{K}} \mathrm{PTN}, \underline{\mathrm{ITFG}} 2, \underline{\mathrm{C}} 12$ orf66, and $\underline{\mathrm{S}} \mathrm{TT} 2$-containing regulator of mTORC1 \\
\hline LAMP1 & lysosomal associated membrane protein 1 \\
\hline LC3 - II & microtubule-associate protein 1 light-chain 3 \\
\hline LDL & low-density lipoprotein \\
\hline LKB1 & liver kinase B1 \\
\hline LRP & lipoprotein receptor-related protein \\
\hline MAPK & mitogen activated protein kinase \\
\hline MAPKK & mitogen activated protein kinase kinase \\
\hline MCL1 & myeloid cell leukemia sequence 1 \\
\hline MDR1 & multidrug resistance 1 \\
\hline
\end{tabular}




\begin{tabular}{|c|c|}
\hline MEK & mitogen activated protein kinase kinase \\
\hline MLCK & myosin light-chain kinase \\
\hline mLST8 & mammalian lethal with Sec13 protein 8 \\
\hline mTORC1 & mechanistic target of rapamycin complex 1 \\
\hline mTORC2 & mechanistic target of rapamycin complex 2 \\
\hline MVB & multivesicular bodies \\
\hline NAD + & nicotinamide adenine dinucleotide + \\
\hline NADH & nicotinamide adenine dinucleotide hydride \\
\hline $\mathrm{NE}$ & nuclear envelope \\
\hline NES & nuclear export sequence \\
\hline $\mathrm{NF \kappa B}$ & nuclear factor $\kappa \mathrm{B}$ \\
\hline NLS & nuclear localization sequence \\
\hline NLRP3 & leucine rich repeat and pyrin domain like receptor like 3 \\
\hline NPC & nuclear pore complex \\
\hline NPRL2 & NPR2 like, GATOR1 complex subunit \\
\hline NPRL3 & NPR3 like, GATOR1 complex subunit \\
\hline NSCLC & non small cell lung cancer \\
\hline OGT & O-linked $\beta$-N-acetylglucosamine transferase \\
\hline P53 & tumor protein 53 \\
\hline P150 & protein 150 \\
\hline P90RSK & p90 ribosomal S6 kinase \\
\hline PDK1 & 3-phosphoinositide-dependent protein kinase-1 \\
\hline PFK & phosphor-fructo kinase \\
\hline
\end{tabular}




$\begin{array}{ll}\text { PH } & \text { pleckstrin homology } \\ \text { PHGDH } & \text { 3-phosphoglyerate dehydrogenase } \\ \text { PI3K } & \text { phosphatidylinositol 3-kinase } \\ \text { PI(4,5)P2 } & \text { phosphatidylinositol-(4,5)-bisphosphate } \\ \text { PI(3,4,5)P3 } & \text { phosphatidylinositol-(3,4,5)-trisphosphophate } \\ \text { PKA } & \text { protein kinase A } \\ \text { PKC } & \text { protein kinase C } \\ \text { PKD } & \text { protein kinase D } \\ \text { PLC } 11 & \text { phospholipase C gamma 1 } \\ \text { PRAS40 } & \text { proline-rich Akt substrate of 40 kDa } \\ \text { PTB } & \text { phosphotyrosine binding } \\ \text { PTEN } & \text { phosphatase and tensin homolog } \\ \text { PTM } & \text { post translational modification } \\ \text { PPP } & \text { pentose phosphate pathway } \\ \text { Rab } & \text { reactive oxygen species } \\ \text { Raf } & \text { ras-related protein } \\ \text { RAS-GRP1 } & \text { Raf proto-oncogene, serine/threonine kinase } \\ \text { Rag } & \text { ras guanyl releasing protein 1 } \\ \text { Raptor } & \text { Ras homolated GTP-binding protein } \\ \text { Rheb } & \text { Rictor }\end{array}$


RTK

S6K1

SAMTOR

SH2

$\mathrm{SH} 3$

SHIP2

SIM

SLC38A9

Snail

SOS

Src

SREBP

Stat3

TERT

$\mathrm{TF}$

TFEB

TKI

TNBC

TOM1L1

TOS

TRAF

TRAIL

TRIF receptor tyrosine kinase

p70S6 kinase 1

S-adenosylmethionine sensor upstream of mTORC1

src homology 2

src homology 3

inositol polyphosphate 5-phosphatase 2

structured illumination microscopy

solute carrier family 38 member 1

Zinc finger protein SNAI1

son of sevenless

Src proto-oncogene, non-receptor tyrosine kinase

sterol regulatory element-binding protein

signal transducer and activator of transcription 3

telomerase reverse transcriptase

transcription factor

transcription factor EB

tyrosine kinase inhibitor

triple negative breast cancer

Target of Myb1 like 1 Membrane trafficking protein

TOR signalling motif

tumor necrosis factor receptor-associated factor

tumor necrosis factor-related apoptosis-inducing ligand

TIR-domain-containing adapter-inducing interferon- $\beta$ 
TSC1

TSC2

TWIST1

ULK

V-ATPase

VEGF

VHL

XIAP
TSC complex subunit 1

TSC complex subunit 2

twist related protein 1

Unc-51 like autophagy activating kinase

Vacuolar-type H+ - ATPase

vascular endothelial growth factor

von Hippel-Lindau

$\mathrm{x}$-linked inhibitor of apoptosis 
Chapter 1: Introduction 


\subsubsection{The adaptability of human physiology}

The human body is a complex system and each cell is constantly exposed to different environmental stimuli. Cells generally adapt quickly and efficiently to different environmental exposures to prevent problems for the overall health of the body. For example, during exercise, breathing rate and heart rate must increase blood flow towards parts of the body that require oxygen for energy generation (Joyner and Casey, 2015). During alcohol consumption, the liver upregulates detoxification mechanisms to break down alcohol and prevent toxic chemical intermediates from harming different functions within the body (Osna et al., 2017). When the human body is exposed to different viruses and harmful bacteria, the immune system must quickly respond and remove the harmful pathogens to prevent illness (Marshall et al., 2018). Hence, to respond to different challenges from the environment, cells within body must sense and alter specific mechanisms at the molecular level to modulate their physiology that allow both cellular and systemic adaption. At the molecular level, cells respond to environmental changes through protein receptors and internal metabolic sensors.

Cells sense their environment in many different ways, two of the most important being: chemical signals from other cells (e.g. growth factors) and direct sensing of the environment (e.g. stressors and nutrients). Chemical signals (some of which can be hormones, growth factors and cytokines) are important mediators of communication between cells, essential for a variety of biological processes including organ function, cell growth and division, and surveillance of pathogens. For example, a small chemical signal or ligand called epidermal growth factor (EGF), is a molecule that is produced by many different types of cells, and when released, it interacts 
with receptors on the same cell or other nearby cells, leading to changes in cell physiology such as proliferation, migration, and survival (Singh and Harris, 2005).

Cells also directly sense their environment by detecting various forms of stressors and by monitoring nutrient availability. Cell stressors are agents which may negatively impact the cells ability to survive, grow, and divide. There are different types of stressors, including heat shock (temperatures exceed normal than average), DNA damage (of which there are many kinds), and production of harmful chemicals that disrupt both DNA, proteins and other molecules, such as reactive oxygen species (ROS) (oxygen by products produced via different internal mechanisms) (Fulda et al., 2010). Cells also directly survey their environment by assessing nutrient availability, as the supply of various specific nutrients can vary both under normal and pathological physiological conditions. For example, cells possess various sensor systems that monitor the availability of glucose (and some of its specific metabolites) and amino acids. Some sensor systems include proteins that sense growth factors (e.g. Epidermal growth factor receptor (EGFR) and environmental/metabolic stress sensors (e.g. AMP-activated protein kinase (AMPK), mechanistic target of rapamycin complex 1 (mTORC1), and mitogen activated protein kinase (MAPK), all of which will be discussed further in sections 1.1.2, 1.1.3, 1.3, and 1.4. Both glucose and amino acids are important molecules that supply the cell with energy and building blocks for the generation cellular biomolecules (Boroughs and DeBardinis, 2015). Low availability of nutrients may negatively impact the cells ability to proliferate, grow, divide, and survive. It is important that cells sense their environment and modulate specific aspects of their physiology to adapt or to respond to environmental changes. Disruption of cellular environmental sensing mechanisms can lead to various forms of disease, either as a result of 
uncontrolled growth, proliferation and survival (in diseases like cancer) or aberrant tissue disruption due to do failure of cellular adaptation to environmental conditions (in disease like diabetes).

Cancer is one of the leading causes of death in Canada, where 1 in 2 Canadians will be diagnosed with cancer during their lifetime, including an estimated 206, 200 diagnosis of new cases in 2017, while 1 in 4 Canadians are expected to die from cancer (Smith et al., 2018). Cancer is defined as the uncontrolled growth, division, and proliferation of cells, that if left untreated will lead to problems in organ function, and in many cases lead to death (Hassanpour and Dehghani 2017; Blackadar 2016). Cancer is a complex and heterogeneous disease, which can either initiate in or spread to a wide range of tissues within the body, some including lung, prostate, bone, brain and breast. Many factors contribute to increased incidence of cancer, including exposure to chemical compounds, virus, bacteria, and radiation (Hassanpour and Dehghani 2017; Hanahan and Weinberg, 2011). Cancer initiation occurs in response to a number of successive gene mutations, leading to abnormal function of proteins in the cell, specifically resulting in impairment of cellular physiology to favour uncontrolled growth, survival, and proliferation (Hassanpour and Dehghani 2017).

Several mutations have to occur within the same cell to trigger cancer initiation, suggesting that one of the early mutations may impact genes involved in DNA repair or genome stability. Indeed, many studies have found that cancer cells have impaired DNA repair systems (Jeggo et al., 2016). In contrast to normal cells, in which DNA damage triggers a cell cycle checkpoint to allow repair of the damaged DNA before continuing with the cell cycle (or in some 
cases programmed cell death or apoptosis), many cancer cells harbor mutations in the genes required to arrest the cell cycle in response to DNA damage, or in the DNA damage repair machinery (Jeggo et al., 2016; Turgeon et al., 2018). Unrepaired DNA damage, such as that in certain cancer cells, can lead to genome instability, increasing the chances of further mutation, therefore increase the acquisition of uncontrolled growth, survival and proliferation capabilities in cancer cells (Jeggo et al., 2016; Turgeon et al., 2018). One of the major mechanisms by which cancer cells exhibit an increase in uncontrolled growth, survival and proliferation, is by accumulating mutations in (or otherwise exhibiting disruptions in the normal regulation of) genes involved in environmental sensing mechanisms, one of which is in the proteins that bind to the small chemical hormone signals like EGF, called EGFR.

\subsubsection{Sensing signals from other cells: Receptors as sensors of intercellular communication}

Cells responds to extracellular changes in the environment by adjusting internal mechanisms according to the stimuli, leading to alterations in cellular physiology. The cell contains a surface membrane lipid bilayer, that acts as a physical barrier between the environment and its internal molecular mechanisms, organelles, and genetic data (Harayama and Riezman, 2018). Because the cell contains a lipid bilayer, cells use surface protein receptors to detect communication from other cells.

Receptors are cell surface proteins that sense and act on extrinsic signals (e.g. ligands including mitogens and growth factors), relaying messages to the inside of the cell, leading to different changes on cellular physiology, including growth, cell death, proliferation, migration, 
and survival. There are many different types of cell surface receptors within the cell, including: 1) receptor tyrosine kinases (RTK) 2) G-protein coupled receptors (GPCR) and 3) immune receptors, each of which modulate different aspects of cellular physiology.

1) Receptor tyrosine kinases (RTK): RTKs are transmembrane cell surface receptor proteins that (for the most part) contain a ligand binding domain, transmembrane domain, and an intracellular kinase domain (Fig. 1.1 and 1.2) (Du and Lovly, 2018; Manning and Toker, 2017; Forrester et el., 2016; Harskamp et al., 2016; Pathi et al., 2016; Lemmon and Schlessinger, 2010; Avruch 2007). There are about $\sim 20$ different classes of RTKs, including ErbB family receptors, insulin receptors, and fibroblast growth factor receptors (FGFR), each respond to distinct and specific extracellular growth factor ligands (Lemmon and Schlessinger, 2010). Each family of receptors have roles in controlling proliferation, growth, survival, and many other aspects of cell physiology (Du and Lovly, 2018; Manning and Toker, 2017; Forrester et el., 2016; Harskamp et al., 2016; Pathi et al., 2016; Lemmon and Schlessinger, 2010; Avruch 2007). When inactive (i.e. not bound to ligands), many RTKs are monomeric, such that upon ligand stimulation, these receptors dimerize and trigger activation of the kinase domains, leading to autophosphorylation of cytoplasmic terminal regions of the receptor (Fig 1.2). This in turn leads to the recruitment of downstream molecules that relay signals to activate several different signalling pathways including PI3K-Akt-mTORC1, Ras-Erk, and PLC (Fig. 1.3) (Du and Lovly, 2018; Manning and Toker, 2017; Forrester et el., 2016; Harskamp et al., 2016; Pathi et al., 2016; Lemmon and Schlessinger, 2010; Avruch 
2007). EGFR is an RTK that will be the focus of my thesis and will be discussed further in section 1.3.

2) G-protein coupled receptors (GPCR): GPCRs are transmembrane receptors that each harbor seven transmembrane domains, and that are coupled to a classical heterotrimeric G protein complex. This G-protein complex is comprised of a GTPase $\mathrm{G} \alpha, \mathrm{G} \beta$ and $\mathrm{G} \gamma$ subunits. (Hilger et al., 2018; Katritch et la., 2013; Kobilka 2007). There are hundreds of different GPCRs, organized into about $\sim 5$ major classes, including the rhodopsin family, adhesion family, frizzled family, glutamate family and secretin family (Kobilka 2007; Fredriksson et al., 2003). Each GPCR family that have different roles, including sensory mechanisms (sight or smell), regulation of adhesion and migration, development, or behavioural and neurological processes such as fight or flight mechanisms (Hilger et al., 2018; Katritch et la., 2013; Kobilka 2007). For example, $\beta_{2}$-adrenergic receptor controls fatty acid beta-oxidation in adipocytes (Johnson 2006). Ligand activation of GPCRs lead to a conformational change in the heterotrimeric $\mathrm{G}$ protein, causing dissociation of the $\mathrm{G} \alpha$ subunit from the G $\beta$ and $\mathrm{G} \gamma$, and activation via GDP exchange for GTP (Hilger et al., 2018; Katritch et la., 2013; Kobilka 2007). G $\alpha$ subunit binds and activates various signalling pathways some including, protein channels, PLC, and adenyl cyclase (Hilger et al., 2018; Katritch et la., 2013; Kobilka 2007). As my thesis will focus on RTKs, GPCRs are not discussed further.

3) Immune receptors: Immune receptors are transmembrane receptors that contain an exterior domain that recognizes pathogens including bacteria, viruses, fungi, parasites, and ligands, transmembrane region, and c-terminal region for signalling (Thaiss et al., 2016; Kawasaki et al., 2014). Immune receptors are categorized into different classes 
based on molecule that is being recognized, which include: 1) Immune receptors that directly sense pathogens or pathogen-derived materials, this includes pattern recognition receptors (e.g. Toll-like receptors) (Kawasaki 2014) and 2) Receptors that sense and bind to cytokines (e.g. Interleukin receptors) that mediate either pro or anti-inflammatory signalling (Thaiss et al., 2016; Garlanda et al., 2013). These various immune receptors are highly expressed in immune cells but are also expressed in other cell types such as epithelial cells (Thaiss et al., 2016; Kawasaki et al., 2014). After engagement of specific ligands (e.g. pathogen-derived molecules or cytokines), various immune receptors recruit and activate signalling pathways including tumor necrosis factor receptor-associated factor (TRAF), TIR-domain-containing adapter-inducing interferon- $\beta$ (TRIF), and nuclear factor $\kappa \mathrm{B}(\mathrm{NF} \kappa \mathrm{B})$, leading to activation of inflammation and clearance of infection (Thaiss et al., 2016; Kawasaki et al., 2014; Xie 2013). For example, nucleotidebinding, oligomerization domain, leucine rich repeat and pyrin domain like receptor like 3 (NLRP3), recognizes pathogen-derived molecule, Pneumolysin secreted by Streptococcus pneumonia, which is important for activating inflammation and clearance of Pneumococcal (McNeela et al., 2010; Harder et al., 2009). Since the focus of this thesis is on RTK signalling, immune receptors will not be discussed further.

RTKs, GPCRs, and immune receptors (with some exceptions within the category of immune receptors, as they contain some classes of internal receptors), are all surface receptors that respond to stimulation at the surface of the cell. As discussed briefly previously, cells also contain internal receptors to sense and respond to changes including nutrient availability (e.g. proteins that assess amino acid levels), and stressors (response to DNA damage, heat shock, and 
ROS). Depending on the levels of nutrient availability or presence of stressors, proteins alter signals that may induce the production of more biomolecules for nutrient replenishment or alter cell physiology to counter stressors by activating mechanisms for cell survival or cell death. Much of the internal sensing mechanisms will be discussed later in section 1.4.1. - 1.4.5., focusing specifically on amino acid and metabolic sensors.

EGFR has been extensively studied, due to the central role that this RTK plays in control of cellular function in many tissues and developmental stages and the key role that EGFR plays as a central driver of tumor progression in many forms of cancer (Sigismund et al., 2018). However, EGFR function is complex and there remains an incomplete understanding of EGFR regulation, and how disruption of EGFR function can impact different forms of cancer. Many studies have highlighted that dysregulation in EGFR function in cancer can occur due to genome disruption that often leads to activating mutation of EGFR or copy number amplification (Sigismund et al., 2018). In addition, other non-genetic disruption of EGFR regulation, such as the increased translation of EGFR triggered during hypoxia, can lead to aberrant elevated EGFR function in cancer cells (Franovic et al., 2007). This disrupted function in EGFR can promote tumor growth and progression by shifting cells into a hyper-proliferative or invasive state, as a result of modulation of cellular mechanisms to promote proliferation, survival, metabolism, and growth (Sigismund et al., 2018).

EGFR disruption and the different phenotypes that follow have been highlighted in different forms of cancer, some including $\sim 40-60 \%$ glioblastomas (Binder et al., 2018; Maire et al., 2014; Furnari et al., 2007), 40\% head and neck non small cell lung cancers (NSCLC) 
(Midha et al., 2014), and $20 \%$ triple negative breast cancers (Du and Lovly, 2018; Sigismund et al., 2018; Kalimutho et al., 2015). There are different drugs that have been developed against EGFR mutations in cancers, which have proven to be successful in the clinic (detail on drug targeting of EGFR will be discussed in section 1.3.5). Thus, EGFR involvement in cancer is very heterogeneous and an important candidate to study its complex regulation. Studying how EGFR mechanisms are controlled contributes important information for the design of future therapies and treatments against cancer. EGFR signalling mechanisms and molecular activity will be further discussed in section 1.3.1. - 1.3.6., highlighting its contribution to signalling towards downstream substrates. While EGFR and other receptors are mediators for sensing cues from other cells from the external environment, internal mechanisms also sense environmental changes for the availability of nutrients. Importantly, the extracellular cues derived from intercellular communication must be integrated with intracellular signalling (e.g. stress, nutrients) in order to establish a coordinated cellular response. Accordingly, cells contain intracellular protein signalling machinery, such as mechanistic target of rapamycin complex 1 (mTORC1), that act as a sensor for nutrients and energy supply. Furthermore, mTORC1 is also regulated by cell surface receptors such as EGFR. mTORC1, in turn alters cell physiology according to the levels of nutrients/energy available and to receptor stimulation, which I discuss next.

\section{$\underline{1.1 .3 \text { Internal sensing: Intracellular sensor in metabolism }}$}

Metabolism is a series of dynamic, interlinked processes that include catabolic (disassembly of material for energy and biomolecules) and anabolic (generation of complex 
macromolecules or biomass) pathways, important for the regulation of cellular physiology (Valvezan and Manning, 2019). This complex network of metabolic signals must be regulated, in order to both meet the requirements of the cell (e.g. for generation of complex macromolecules such as proteins or DNA) and to meet the nutritional capabilities of the cell (supply of amino acids, nucleotides). mTORC1 is an integrator of different signalling pathways and in doing so is an important sensor of cellular energy, nutrient, and metabolite availability (Valvezan and Manning, 2019). For example, in the perspective of amino acids, mTORC1 is activated when amino acids are available within the cell, while mTORC1 inactivation occurs under amino acid insufficiency (Valvezan and Manning, 2019; Saxton and Sabatini, 2017). In turn, mTORC1 is a major regulator of many different metabolic pathways, and in doing so acts as a major switch or hub to balance catabolic and anabolic pathways during varying environmental conditions.

The important role of mTORC1 in sensing and controlling the cells metabolic dynamics is made evident by the many lines of evidence that show disruptions in mTORC1 activity are linked to different diseases, including, Parkinson's disease, Alzheimer's disease, and cancer (Saxton and Sabatini, 2017). The regulation of mTORC1 will be further discussed in section 1.4.1. - 1.4.5., highlighting nutrient sensing of amino acids/energy and the integration of signals on mTORC1 to control its activity.

\subsubsection{Introduction: Hallmarks of cancer}

As described in a seminal review article by Hanahan and Weinberg in 2000 (and updated in 2011), cancers share six hallmarks or features that appear in cancer cells or within the tumor 
microenvironment (Hanahan and Weinberg, 2011; Hanahan and Weinberg, 2000). The six hallmarks of cancer based on Hanahan and Weinberg include, 1) sustaining proliferative signalling, 2) evading growth suppressors, 3) activating invasion and metastasis, 4) enabling replicative immortality, 5) inducing angiogenesis, and 6) resistance to cell death, which are all briefly described below.

1) Sustaining proliferative signalling: Healthy cells precisely regulate the release of chemical signals (e.g. growth factors and mitogens) and their subsequent stimulation of receptors to activate proliferative and growth pathways under right regulation. While many different mutations can lead to sustained proliferative signalling, many cancer cells lose appropriate regulation of receptor activation, disrupting function leading to constitutive proliferation and unnecessary cell cycle initiation. Proliferation is normally regulated by control of cell cycle for G0/G1 and G1/S transition. After mitogen stimulation, receptors trigger downstream signals to PI3K-Akt and MAPK pathways that activate the cyclin D1/cyclin-dependent kinase 4/6 (CDK 4/6) complex (Wee and Wang, 2017). The cyclin D1-CDK4/6 complex drives cells to go through G1 and subsequently, leading to the inhibition of retinoblastoma protein (RB) and activation of transcription factor E2F for G1/S-phase progression (Otto and Sicinski, 2017; Wee and Wang, 2017). In cancer cells, losing control of activity to promote sustainable proliferation include triggering of constitutive release hormones causing stimulation of self and surrounding tumor microenvironment, increasing the expression of receptors to enhance sensitivity, and hyper activation (Hanahan and Weinberg, 2011; Lemmon and Schlessinger, 2010; Witsch et al., 2010; Perona 2006). 
In different forms of cancer that contain an RB deletion, cell cycle progression occurs independently of cyclin D1-CDK4/6 activity (Otto and Sicinski, 2017; Fletcher et al., 2010).

2) Evading growth suppressors: Cell growth is a precise and tightly regulated process, while cancer cells have evolved mechanisms to grow and divided uncontrollably by impairing function of growth suppressor genes or tumor suppressor genes. Cancer cells down regulate or lose function of tumor suppressor genes, leading to pro-growth pathways (Hanahan and Weinberg, 2011). An important growth suppressor is phosphatase and tensin homolog (PTEN), which functions to balance and counteract the production of phosphatidylinositol 3,4,5 trisphosphate ( $\mathrm{PI}_{345 \mathrm{P}_{3}}$ ) (a product produced in the PI3K pathway, details discussion in section 1.3.2.) by

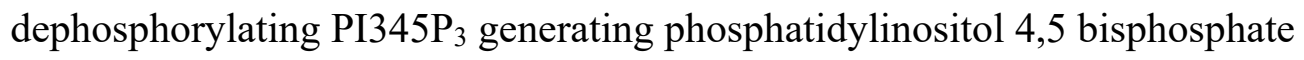
(PI45 $\mathrm{P}_{2}$ ) (Maehama and Dixon, 1998). $\mathrm{PI} 345 \mathrm{P}_{2}$ is an important secondary messenger that activates growth pathways including mTORC119o0 (detailed discussion in section 1.4.1-1.4.5) and Akt (detailed discussion in section 1.3.2) (Saxton and Sabatini, 2017). In prostate cancers containing deletion in PTEN, Akt activity increased, leading to the expression of C-X-C chemokine receptor type 4 (CXCR4) and C-X-C motif chemokine ligand 12 (CXCL12) (Conley-LaComb et al., 2013). Furthermore, the increase in CXCL12 and CXCR4 are important factors that contribute to tumor growth progression (Conley-LaComb et al., 2013).

3) Activating invasion and metastasis: Migration is a tightly maintained mechanism performed by cells that is important for processes of wound repair and morphogenesis/development (Welf and Haugh, 2011). In normal epithelium, the 
structure of cells within tissues is maintained in a non-migratory state due to Gap and tight junctions between cells, and integrin-dependent interactions of epithelial cells and the basal lamina (Zihni et al., 2016; Harburger and Calderwood, 2009; Meşe et al., 2007). Interestingly, the vast majority of solid tumors are of epithelial origin (Nelson and Guyer, 2014). In a process called epithelial to mesenchymal transition (EMT), cells increase invasive behaviour, often resulting in alterations in cellular differentiation state, where epithelial markers are down regulated (e.g. decrease expression of tight junctions and Gap junctions), while mesenchymal markers are increased (e.g. increasing migratory pathways and metabolic pathways) (Mittal 2018). This change is a result of transcriptional reprograming often associated with the activity of transcription factors including Zinc finger protein SNAL1 (Snail) and twist related protein (Twist1) (Snail will be discussed in further detail in section 1.7.1) (Mittal 2018, Wang et al., 2013). Thus, cancers may take advantage of EMT by using Snail to alternate the expression of genes that down regulates adherens junctions (e.g. E-cadherin) and promote tumor invasion and motility (Hanahan and Weinberg, 2011; Peinado 2004).

4) Enabling replicative immortality: In normal cells, there are mechanisms that limit the amount of successive cell divisions before permitted into a state of senescence; a process by which, cells irreversibly enter a non-proliferation state but are still viable (Wei and Ji, 2018). Telomeres are repeats of nucleotides (TTAGGG) found at the end of chromosomes and are elongated by an enzyme called telomerase (Blasco 2005). Telomeres shorten in somatic cells with each cell division and shortened telomeres trigger senescence (Wei and Ji, 2018; Hanahan and Weinberg, 2011; Blasco 2005). In 
different cancers, mutations in the telomerase reverse transcriptase (TERT) promoter, results in upregulation of transcription and subsequent hyperactivation of telomerase, leading to replicative immortality (Liu et al., 2016).

5) Inducing angiogenesis: Angiogenesis is the process of generating new blood vessels from pre-existing blood vessels (De Palma et al., 2017), an important process during stages of development, embryogenesis, and wound healing (De Palma et al., 2017; Hanahan and Weinberg, 2011). Cancer cells rapidly divide and this occurs outside of normal developmental programs. As a result, the rapid overgrowth of cells produces a tumor that can have areas that are poorly vascularized to begin with, causing hypoxia and nutrient deprivation (Muz et al., 2015). Some cancer cells trigger signalling that leads to enhanced angiogenesis (Muz et al., 2015; Hanahan and Weinberg, 2011; Kazerounian et al., 2008). For example, cancer cells sense hypoxia via hypoxia inducible factor $1 \alpha(\mathrm{HIF} 1 \alpha)$, followed by changes in gene expression, in particular the increased expression of vascular endothelial growth factor (VEGF), triggering the growth and expansion of endothelial cells that produce new and expanded blood vessels (Muz et al., 2015; Hanahan and Weinberg, 2011; Kazerounian et al., 2008). This contributes to allowing cancer cells to gain supply of nutrients contributed by the newly formed vasculature (Muz et al., 2015; Hanahan and Weinberg, 2011; Kazerounian et al., 2008).

6) Resistance to cell death: Programmed cell death or apoptosis is an important process that is triggered in different stages of a cells life cycle, including tissue development, exposure to pathogens (e.g. bacteria or viruses), or DNA damage that cannot result in repair (Turgeon et al., 2018; Tan et al., 2015). Tumor protein 53 (p53) is involved in 
signalling pathways that respond to different cell stresses (e.g. DNA damage) that mediate changes to transcriptional activity to trigger senescence, apoptosis, and cell cycle arrest (Muller et al., 2013; Junttila et al., 2009). In some cancers, mutations in p53 inhibit surveillance mechanisms to detect DNA damage and blunt DNA repair mechanisms by preventing the activation of ataxia-telangiectasia mutated (ATM) kinase (kinase involved in DNA repair mechanisms) (Liu et al., 2010; Song et al., 2007). Furthermore, due to mutations in p53, programmed cell death mechanisms are impaired and therefore proceed into a proliferate state (Liu et al., 2010; Song et al., 2007).

Recently after Hanahan and Weinberg's review, new hallmarks of cancer have emerged including themes of deregulation of cellular energetics and metabolism, tumor-promoting inflammation, and the ability of cancer cells to avoid immune destruction (Pavlova and Thompson, 2016; Vinay et al., 2015). Cancer is linked to changes in cell metabolism, where cancer cells shift and adapt their metabolic state to promote cancer cell proliferation or to promote survival given the tumor microenvironment. Cancer cells adapt their metabolic state by modifying the usage of glycolysis and increasing the energy demand by increase oxidative phosphorylation, as I describe in detail below.

\subsubsection{Emerging concepts for Hallmarks of cancer: metabolism and Warburg effect}

A phenomenon observed by physiologist Otto Warburg, described that cancer cells consumed more glucose and produced more lactate, even in the presence of oxygen (now termed 
"Warburg effect”) (Warburg et al.,, 1924; Warburg et al., 1927). Furthermore, Otto Warburg also observed that cancer cells used glycolysis more compared to oxidative phosphorylation (Warburg 1956; Warburg et al., 1927; Yoshida 2015), which seemed contradictory, as oxidative phosphorylation produces more ATP per glucose molecule. Glycolysis produces pyruvate from glucose, which in the presence of oxygen typically enters the Kreb's cycle as acetyl-CoA, which then allows production of ATP during oxidative phosphorylation (Lunt and Vander Heiden, 2011). In contrast, during oxidative glycolysis, pyruvate is converted to lactate, along with the production of nicotinamide adenine dinucleotide $+(\mathrm{NAD}+)$ from nicotinamide adenine dinucleotide hydride (NADH) (Lunt and Vander Heiden, 2011). The process of the Warburg effect is a very complicated concept and it is still not completely understood why cancer cells utilize this less efficient mechanism for producing energy. Since this initial discovery, many have proposed different mechanisms as to why cancer cells undergo the Warburg effect. A mechanism proposed by Liberti and Locasale in 2016, describes that cancer cells use the Warburg effect to focus on the production of NAD + molecules that allows constitutive upregulation of the glycolysis pathway (Liberti and Locasale, 2016). NAD+ is an important cofactor that is produced by many metabolic pathways but in particular for glycolysis, as an end product (Lunt and Vander Heiden, 2011). Interestingly, the end product NAD+, is able to shuttle back into glycolysis and contribute to the reaction catalyzed by glyceraldehyde 3-phosphate dehydrogenase within glycolysis, allowing glycolysis to continue the production of more ATP (Fig 1.4) (Liberti and Localsale, 2016; Lunt and Vander Heiden, 2011). Furthermore, another possibility as to why the Warburg effect occurs, glycolysis produces less ATP from a single glucose molecule but does so more quickly than oxidative phosphorylation, but, ATP is not often limiting in a cancer cell (Epstein et al., 2014; Locasale and Cantley, 2011; Heiden et al., 2010). It 
is also possible that glycolysis serves to provide not just metabolites for ATP production, but also provides intermediates for other metabolic pathways that deviate from the core glycolysis pathway at specific branch points, such as the hexosamine biosynthetic pathway, the pentose phosphate pathway and the serine biosynthetic pathway (which will be discussed in section 1.2.3) (Ward and Thompson, 2012; Locasale and Cantley, 2011; Heiden et al., 2010), but removing metabolites from glycolysis does not lead to lactate production (Lunt and Heiden, 2011). In contrast to the Warburg effect, other mechanisms have been described that observe cancer cells to strategically "borrow" glycolysis intermediates to siphon them to other metabolic pathways important for biomass production, leading towards proliferation and expansion of new cancer cells. Cancer cells have unique metabolism and unique metabolic need.

\subsubsection{Cancer metabolism: Glycolysis-associated pathways fuel biomass production}

Cancer cells have the ability to shift their metabolic state by "borrowing" intermediates of glycolysis that are then siphoned to different biosynthetic pathways for the formation of biomolecules (Pavlova and Thompson, 2016; Vander Heiden et al., 2009). The formation of biomolecules allows for the generation of structural cellular components including, nucleotides, glycans and amino acids, important for the expansion of cancer cells (Pavlova and Thompson, 2016). Glycolysis is a multistep process that contains key regulatory enzymes that are required for conversion and transformation of glucose into different intermediates (Fig 1.4) (Lunt and Vander Heiden, 2011). The goal of glycolysis is to generate energetic carrier molecules in the form of ATP and an end product of pyruvate, which is used to generate more yield of ATP (compared to glycolysis) upon entering the Kreb's cycle and then subsequently engagement of 
the process of oxidative phosphorylation (Fig 1.4) (Lunt and Vander Heiden, 2011). In addition to generating these energetic molecules and as mentioned briefly above, glycolytic intermediates may branch into different biosynthetic pathways (Lunt and Vander Heiden, 2011). The branching of intermediates occurs within all cells, but cancer cells may be more dependent on branched pathways to proliferate and expand, given their need for biomass production to fuel cell growth and division.

Glycolysis intermediates may be used for other biosynthetic pathways that generate nucleotides, amino acids, and glycans. In many cases for cancer, cells develop metabolic adaptations that are more dependent on biosynthetic pathways for proliferation, since in many cases cancer cells within poorly vascularized tumor microenvironments are deficient in extracellular sources of these biomolecules (Pavlova and Thompson, 2016). Therefore, glycolysis may be exploited for intermediates using them to generate key biomolecules that may be used for structural components important for the expansion of cancer cells (Pavlova and Thompson, 2016). In the pentose phosphate pathway (PPP), (which branches off from glucose 6 phosphate or fructose 6 phosphate from glycolysis) cells generate nucleotides for the formation monomers for deoxyribonucleic acid (DNA) and ribonucleic acid (RNA) (Lunt and Vander Heiden, 2011). In some cancers, cells upregulate PPP, and require the glycolysis intermediate glucose 6 phosphate (G6P) to generate nucleotides (Fig 1.4) (Wang et al., 2011; Xu et al., 2009; Ying et al., 2012). In some cases of melanoma and breast cancer cells, amino acid metabolism pathways are upregulated, requiring the diversion of 3-phosphoglycerate from the glycolytic pathway to amino acid biosynthetic pathways for serine and glycine biosynthesis (Fig 1.4) (Locasale et al., 2011). In another aspect of carbohydrate metabolism, cells use carbohydrates 
(also known as polysaccharides) to modify molecules like proteins and lipids to ensure the correct operation of important cellular processes, including signalling, protein stability, and protein-protein interactions (Varki 2017). Importantly, the hexosamine pathway uses fructose 6 phosphate (F6P) from glycolysis for the production of N-acetylglucosamine-6-phosphate (GlcNAc-6-P), a glycan substrate important for protein stability and function (Fig 1.4) (Varki 2017; Hanover et al., 2010). In some cancers, a metabolic shift allows metabolic enzymes to divert more glycolytic resources for the production GlcNAc-6-P to meet cellular needs (Pavlova and Thompson, 2016; Itkonen et al., 2013). Therefore, cancer will exploit F6P from glycolysis, generating GlcNAc-6-P substrates used for protein stability and function, fueling the high demand for nucleotides that is required for DNA replication and cell cycle progression in rapidly proliferating cancer cells (Pavlova and Thompson, 2016; Itkonen et al., 2013).

These examples demonstrate how cancer cells shift metabolism by exploiting pathways that utilize intermediates that branch off of the "main" glycolytic pathway for the production of different biomolecules for increased biomass and proliferation potential. It is therefore critical to consider the cellular pathways that ensure regulation, including at the transcriptional level, but also control of metabolic enzymes by allosteric regulation and translational modulation that increase biomass production. A key regulator of cell metabolism, important for enhancing metabolic pathways that increase biomass production, is c-Myc, and I will discuss how this transcription factor is regulated by $\mathrm{mTORC} 1$ and other proteins as a central focus of this thesis. Interestingly, cancers not only exploit metabolic pathways for biosynthesis pathways but also use them to protect against therapeutics and therapies for survival, as I will examine next. 


\subsubsection{Cancer metabolism: Oxidative phosphorylation and therapeutic resistance}

The Warburg effect and the exploitation of glycolysis are two mechanisms of metabolic adaptations used by some types of cancer cells to adapt to the tumor microenvironment, in a manner that is thought to increase the production ATP and/or biomass. However, cancer cells exhibit heterogeneity for their metabolism, which may reflect that tumors are made up of distinct subpopulations of metabolically-distinct cancer cells, or that cancer cells are able to shift or adapt their metabolism from one state (e.g. Warburg-like metabolism) to other states. Indeed, another metabolic state (distinct from a Warburg-like oxidative glycolysis) that some cancer cells utilize, is the strong and important reliance on oxidative phosphorylation for ATP production, a state that has been linked to resistance to cancer therapeutics.

As mentioned above, two key mechanisms the cell uses to generate ATP are glycolysis and Kreb's cycle/oxidative phosphorylation (Lunt and Vander Heiden, 2011). But among the two energy pathways, oxidative phosphorylation yields more molecules of ATP compared to glycolysis (Lunt and Vander Heiden, 2011), making the use of Kreb's cycle and oxidative phosphorylation an interesting strategy that cancer cells may use to increase energy potential for proliferation. Many studies have now reported that some cancer cells exhibit oxidative metabolism of glucose, such as Hodgkin lymphoma cells and cancer stem cells (CSC), that had both oxidative phosphorylation and electron transport chain genes upregulated, increased mitochondrial biomass, and increased ATP production by oxidative phosphorylation compared to non-oxidative processes (Batlle and Clevers, 2017; Birkenmeier et al., 2016). The reliance on oxidative metabolism is a hallmark of cancer stem cells in many different types of cancer (Batlle 
and Clevers, 2017). Since cancer stem cells do not undergo rapid proliferation, and the rapidly proliferating cells may instead rely on the Warburg-like oxidative glycolysis. Thus, shifting the metabolic capacity towards oxidative phosphorylation process and less importance on glycolysis pathways (e.g. resulting in lactate production) for ATP generation may enhance growth and survival of some cancer cells (Batlle and Clevers, 2017; Birkenmeier et al., 2016).

Interestingly, the utilization of oxidative phosphorylation by some cancer cells also allows the production of reactive oxygen species (ROS), which has been reported to function as means of drug resistance to promote survival. In triple negative breast cancer (TNBC) stem cells, MYC and MCL1 genes are amplified, which caused an increase to mitochondrial biogenesis and oxidative phosphorylation, which in turn leads to enhanced production of ROS, which subsequently upregulates Hypoxia inducible factor $1 \alpha$ (HIF-1 $\alpha)$ (Lee et al., 2017). Production of ROS and upregulation in HIF-1 $\alpha$, leads to the generation of chemotherapy resistance to chemotherapy drug paclitaxel (Lee et al., 2017). Thus, some cancer cells are able to metabolically reprogram themselves by shifting their metabolic state to conditions that may favour the production of biomolecules for cancer cell proliferation or upregulate different pathways in oxidative phosphorylation for increased survival or therapeutic resistance.

As such, cancer cells are now well known to depend on various metabolic adaptions and alterations to ensure survival and to enhance proliferation. This ability to shift metabolic activities relies in part on metabolic sensing, which a cell has to integrate with mitogenic signals to promote growth, survival, and proliferation. Mechanisms of mitogenic signalling will be 
discussed for EGFR, which will be followed by the regulation of metabolic sensing examined by mTORC1.

\subsubsection{ErbB family and regulation}

The regulation of different aspects of cell physiology requires maintenance and control by specific proteins within the cell. An important category of RTKs called the ErbB family controls numerous cellular processes such as proliferation, growth, metabolism, cell survival and many others (Lemmon and Schlessinger, 2010). With these cellular processes, the ErbB member, EGFR is implicated in many types of cancers. It is important to understand the dynamic activity and regulation of the ErbB family of receptors from the initial activation to deactivation. Hence, understanding how ErbB signalling is disrupted in cancer and how it impacts specific aspects of cancer cell physiology, may improve diagnosis and aid in the development of therapeutic strategies.

EGFR activity is controlled through extracellular binding of its specific ligands such as EGF (Freed et al., 2017; Wilson et al., 2012; Yang et al., 2017; Roepstorff et al., 2009). The binding of these specific ligands to an exofacial domain of the receptor causes a conformational change in which a monomeric EGFR undergoes homo- or hetero- dimerization with other ErbB family of receptors (Lemmon and Schlessinger, 2010) (Fig. 1.2). This dimerization event causes activation of the kinase domain of EGFR, leading to autophosphorylation of tyrosine residues in its c-terminal region of the receptor (Fig. 1.2) (Lemmon et al., 2014; Honegger et al., 2006). These tyrosine phosphorylated residues are part of motifs that become docking sites for 
important domains such as Src homology (SH2) and phosphotyrosine binding (PTB) domains found within downstream signalling enzymes and adaptors (Wagner et al., 2013). As such, these critical proteins such as adaptors and scaffolds are recruited to sites of phosphorylated tyrosine residues (Buday and Tompa, 2010), which will subsequently recruit and lead to activation of protein signalling pathways such as Gab1/PI3K/Akt/mTORC1 (Dibble and Cantley, 2015), Ras/Raf/Erk (Morrison 2012), PLC $\gamma 1$-PKC (Nakamura and Fukami, 2017), and many others (Fig. 1.3). Each of these signalling pathways control different aspects of cellular physiology, including, apoptosis, cell survival, proliferation, metabolism, DNA transcription or translation, and many others. Three pathways that will be discussed in detail from EGFR activation: AktmTORC1, PLC $\gamma 1$, and Erk signalling pathways, given the importance of these signals in cancer cell growth, proliferation and survival.

\subsubsection{EGFR activation of Akt-mTORC1 pathway}

An important signalling protein activated by EGFR is Akt, which is a serine/threonine kinase that regulates many protein substrates for different physiological responses including survival, proliferation, and migration (Sugiyama et al., 2019). To activate Akt (Fruman et al., 2017; Vanhaesebroeck et al., 2012), specific motifs containing phosphorylated tyrosine residues on EGFR, in particular Y1068 and Y1086 act as docking sites, that recruit scaffold proteins growth factor receptor-bound protein 2 (Grb2) via SH2 domain, followed by recruitment and activation via phosphorylation of Grb2 associated binder protein 1 to proline rich domain (Gab1) via Grb2 SH3 domain (Fruman et al., 2017; Vanhaesebroeck et al., 2012; Lock et al., 2000). Phosphorylated residues on Gab1 act as docking sites to recruit the class IA phosphatidylinositol 
3 kinase (PI3K) via its $\mathrm{p} 85$ subunit, which triggers a conformational change activating the catalytic activity of the p110 subunit (Fruman et al., 2017; Vanhaesebroeck et al., 2012). This enhanced activity of PI3K converts the phosphoinositide species phosphatidylinositol 4, 5 bisphosphate ( $\left.\mathrm{PI}_{45} \mathrm{P}_{2}\right)$ to phosphatidylinositol-3, 4, 5-trisphosphate (PI345P 3 ) (Fig. 1.5) (Fruman et al., 2017; Vanhaesebroeck et al., 2012; Kiyatkin et al., 2006; Mattoon et al., 2004; Holgado-

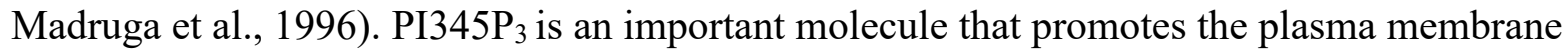
recruitment and activation of Akt via its pleckstrin homology $(\mathrm{PH})$ domain (Manning and Toker, 2017). Recruitment of Akt to the plasma membrane allows for its phosphorylation in serine 473 and threonine 308, mediated by mTORC2 (Sarbassov et al., 2005) and PDK1 (Manning and Toker, 2017), respectively, which are both required for full Akt activation (Fig. 1.5). Akt has several isoforms, Akt1, Akt2 and Akt3, each of which are activated via different mechanisms (Sugiyama et al., 2019). Akt1 and Akt3 are activated by $\mathrm{PI} 345 \mathrm{P}_{3}$ primarily at the plasma membrane, while Akt2 is activated by phosphatidylinositol-3,4-bisphosphate ( $\left.\mathrm{PI}_{3} 4 \mathrm{P}_{2}\right)$ (produced from $\mathrm{PI} 345 \mathrm{P}_{3}$ through the activity of SH2 domain containing inositol polyphosphate 5phosphatase 2 (SHIP2), at both the plasma membrane and endosomes (Sugiyama et al., 2019; Liu et al., 2018). Furthermore, different Akt isoforms have non-redundant functions (Sugiyama et al., 2019; Roy et al., 2017; Schultze et al., 2011; Gonzalez and McGraw, 2009; Stambolic and Woodgett, 2006).

Activation of Akt leads to the regulation of many downstream signalling pathways, which I will describe two specific downstream pathways, involving mTORC1 and glycogen synthase kinase 3 (GSK3) signalling and their important in cellular physiology, later in sections 1.6.1. - 1.6.6. Given that Akt controls cell physiology of survival, proliferation, and migration, it 
is apparent that Akt is involved in mechanisms controlling pathways to promote and sustain cancer. An effector of Akt is forkhead box O (FoxO), a transcription factor involved in apoptosis, metabolism and cell cycle (Zhang et al., 2011). Upon activation, Akt phosphorylates and inhibits FoxO activity, while inactive Akt allows FoxO to translocate into the nucleus triggering different signals that activate cellular processes like apoptosis (Zhang et al., 2011). In some cancers, upregulated PI3K pathway leads to the hyper activation of Akt and subsequent inhibition of FoxO (Dansen et al., 2008). Constitutive phosphorylation and thus inhibition of FoxO by upregulated PI3K-Akt signalling allows for cancer cells to avoid apoptosis and sustain survival (Dansen et al., 2008).

In addition, Akt also regulates cell migration and invasion by controlling the formation of invadopodia, which are plasma membrane structures that are generated by specific control of actin dynamics (Eddy et al., 2017). Invadopodia are enriched in matrix metalloproteases within these membrane protrusions, which facilitates the role of invadopodia in migration/invasion (Eddy et al., 2017). In some cancers, PI3K function is impaired increasing formation of PI345 $\mathrm{P}_{3}$, hyper activating Akt activity, upregulating invadopodia formation, and promoting cancer metastasis (Yamaguchi et al., 2011). Both examples demonstrate how impaired control of Akt leads to changes in cellular processes of survival and migration. Another parallel pathway that is activated via EGFR is the phospholipase $\mathrm{C} \gamma 1$ (PLC $\gamma 1$ ) pathway. 


\subsubsection{EGFR activation of PLC $\gamma 1$ pathway}

PLC $\gamma 1$ is also another important protein activated by ligand-bound EGFR (Nakamura and Fukami, 2017). PLC are a family of phospholipid diesterase enzymes that con be further subcatergorize into families of different isoforms PLC $\beta, \operatorname{PLC} \gamma, \operatorname{PLC} \delta, \operatorname{PLC} \varepsilon$, PLC $\eta$, and PLC $\zeta$ (Kadamur and Ross, 2013). PLC contains a core conserved structure which contains a PH domain, four tandem EF hand domains, a split TIM barrel, and a C2 domain (Kadamur and Ross, 2013). Among the different isoforms, PLC $\gamma$ responds to RTK signalling. PLC $\gamma 1$ is specifically activated following phosphorylation by receptor tyrosine kinases or other kinases at Y783 (Sekiya et al., 2004). Phosphorylation of PLC $\gamma 1$ enhances phosphodiesterase activity, which is specific for PI $(4,5)$ P2. As such, PLC $\gamma 1$ activation leads to production of diacylglycerol (DAG) and inositol triphosphate (IP3), both of which become important secondary messengers that will activate other pathways (Fig. 1.6) (Nishizuka 1988; Berridge and Irvine, 1984). DAG interacts and binds to many effector proteins including protein kinase D (PKD), Ras guanyl-releasing protein 1 (RAS-GRP1), Unc-13, and specific isoforms of protein kinase C (PKC) (Almena and Mérida 2011; Cho 2001). IP3 binds to Inositol 1,4,5-triphosphate receptors (IP3R) at the endoplasmic reticulum (ER), which causes the release of intracellular $\left(\mathrm{Ca}^{2+}\right)$ from the lumen of ER into the cytosol (Nishizuka 1988; Berridge and Irvine, 1984). This intracellular $\mathrm{Ca}^{2+}$ then acts as a tertiary signal that binds to a plethora of other substrates, including $\mathrm{PKC}$, calcineurin, dynamin1, amphiphysin, synaptojanin1, eps15, epsin, and AP180 (Saheki and De Camilli, 2012; Cousin and Robinson, 2001; Cousin, 2000; Chen et al., 1999; Slepnev et al., 1998; Bauerfeind et al., 1997; Liu et al., 1994; Nishizuka 1988; Berridge and Irvine, 1984). 
As a result of activation of these $\mathrm{Ca}^{2+}$ and DAG dependent signals, PLC $\gamma 1$ activation leads to cell physiological changes involved in cytoskeleton remodelling, signalling, membrane traffic and dynamics. In muscle cells, $\mathrm{Ca}^{2+}$ signalling is required for the activation of actin filament contraction (Tsai et al., 2015; Clapham 2007). Upon $\mathrm{Ca}^{2+}$ release from the cytosol, $\mathrm{Ca}^{2+}$ binds and activates Calmodulin (CaM) and activates myosin light-chain kinase (MLCK), which in turn activates myosin head light chain (Tsai et al., 2015; Clapham 2007). Activated myosin head light chain causes contraction of muscle via actin filaments (Tsai et al., 2015; Clapham 2007). Importantly, downstream targets of PLC $\gamma 1$ have been highlighted in various forms of cancer. In head and neck squamous carcinomas, increased expression and activity of PKC leads to an increase in cell migration and invasion. This increased activity of PKC has been linked to an increase in signalling by Rho GTPases, which controls cell migration by modulation of actin cytoskeleton remodelling (Pan et al., 2006). In lung cancer, elevated expression of PKC induces mitogen-activated protein kinase kinase (MAPKK or MEK) activation leading to upregulation of proteins $\mathrm{x}$-linked inhibitor of apoptosis (XIAP) and pro-survival protein B-cell lymphoma-extra large (BCL-X $\mathrm{L}_{\mathrm{L}}$ (Pardo et al., 2006). Both examples demonstrate the potential of PLC $\gamma 1$ targets to control different functions in cancer to promote cancer growth, survival and progression. A pathway that is also activated by EGFR is the mitogen-activated protein kinase pathway (MAPK).

\section{$\underline{\text { 1.3.4 EGFR activation of MAPK pathway }}$}

Another parallel signalling pathway that EGFR regulates is the MAPK pathway, which is responsible for controlling cellular processes such as DNA transcription activity, mitosis, and 
growth. Upon EGFR activation, motifs containing phosphotyrosine residues on the EGFR cterminal tail, in particular Y1173 and Y1068, will recruit adaptor proteins SH2-containing protein (SHC) and Grb2, respectively (Fig. 1.7) (Liu et al., 2018; Cargnello and Roux, 2011; Dhillon et al., 2007; Zhang and Liu, 2002). In addition to Grb2 binding and recruitment of Gab1 (as discussed above for PI3K signalling), Grb2 recruits the guanine-nucleotide exchange factor (GEF) protein, son of sevenless (SOS) (Liu et al., 2018; Cargnello and Roux, 2011; Dhillon et al., 2007; Zhang and Liu, 2002). SOS will interact with GDP bound protein Ras and exchange GDP for GTP, activating Ras (Liu et al., 2018; Cargnello and Roux, 2011; Dhillon et al., 2007; Mitin et al., 2005; Zhang and Liu, 2002). Ras has different isoforms including H-Ras, N-Ras, and K-Ras (Fernandez-Medarde and Santos, 2011). Activated Ras binds and activates the serine/threonine kinase Raf, leading to subsequent phosphorylation and activation of the next kinase, MEK (Liu et al., 2018' Cargnello and Roux, 2011; Dhillon et al., 2007; Zhang and Liu, 2002). Activated MEK phosphorylates and activates next serine/threonine kinase, extracellular signal-regulated kinase (Erk), which contains two isoforms p44 and p42 (Fig. 1.7) (Liu et al., 2018; Cargnello and Roux, 2011; Dhillon et al., 2007; Zhang and Liu, 2002). Erk interacts and phosphorylates different effectors within the cell some including p90 ribosomal S6 kinase (RSK) and transcription factors jun, fos and myc, which control proliferation and cell cycle progression (Maik-Rachline et al., 2019; Liu et al., 2018; Cargnello and Roux, 2011; Dhillon et al., 2007; Zhang and Liu, 2002).

Given the involvement of the MAPK pathway in physiological processes including transcription, growth, and mitosis, many studies have examined how disruptions in MAPK pathway may contribute to cancer. Ras is a classic oncogene and mutated in most cancers 
(activating mutation) (Fernandez-Medarde and Santos, 2011). In many cases, Ras mutations often promotes cell cycle progression through the upregulation of myc, fos, jun and other transcription factors that are targets of Ras-Erk signalling pathway. Yong et al in 2011 demonstrated that some breast cancers had upregulated Ras contributing to the strong activation MAPK protein p38 (Yong et al., 2011). Furthermore, activation of p38 MAPK, promotes breast cancer cells to become more migratory and invasive (Yong et al., 2011). In another study by Goueli and Janknecht in 2003, demonstrated that upregulated Ras contributes to the evasion of senescence (Neuzillet et al., 2014; Goueli and Janknecht, 2003). Upregulated Ras activates Erk to stimulate the transcriptional activation of critical telomerase subunit gene for TERT, causing constitutive elongation of telomere repeats, sustaining cell replication and senescence evasion for cancers (Neuzillet et al., 2014; Goueli and Janknecht, 2003). Both demonstrate the importance of regulation of MAPK pathway, especially in impaired functions of cell migration, invasion, senescence and evasion in cancers. Although different signalling pathways and their impaired mechanisms contribute to cancer, many studies have examined EGFR as an important target due to disrupted function in cancer leading to pro-survival, uncontrolled growth, and resistance to many therapeutics.

\subsubsection{EGFR: an oncogene and therapeutic target}

EGFR and the ErbB family of receptors have been shown to be oncogenic and implicated in my cases of cancer that develop resistance to many therapies and treatments. In many cancers, EGFR is disrupted at the genetic level, including the appearance of mutations, and/or the increased expression of EGFR resulting from copy-number amplification, leading to upregulated 
function that support survival, uncontrolled proliferation and growth (Sigismund et al., 2018; Roskoski 2014; Yarden and Pines, 2012).

Since the discovery of EGFR and its contributions to different forms of cancer, there have been a number of therapeutic strategies that have been developed to disrupt EGFR function, in order to down regulate phenotypes of cancer including uncontrolled growth, proliferation, and pro-survival. The available pharmacological drugs that target specific regions of EGFR includes humanized antibody-based drugs (e.g. cetuximab and panitumumab) (Sigismund et al., 2018; Gharwan and Groninger, 2016; Singh et al., 2016; Ciardiello and Tortora, 2008) that bind to the extracellular domain, and specific tyrosine kinase inhibitors (TKI) (e.g. erlotinib, gefitinib, and lapatinib), which target the kinase region of EGFR (Sigismund et al., 2018; Singh et al., 2016). Briefly, in a clinical trial study by Peeters et al. in 2015, it was demonstrated that panitumumab was effective at treating patients with metastatic colorectal cancer, improving patient survival rates and decreased tumor shrinkage versus patients that received placebo therapeutics (Peeters et al., 2015). Furthermore, in a study by Hirsch et al. 2013, a randomized trial examined the effect of either erlotinib or placebo for patients with NSCLC (Hirsch et al., 2013). Erlotinib-treated patients had an increase in survival rates compared to placebo treated patients (Hirsch et al., 2013). Both clinical trials show the effectiveness and potential of EGFR inhibitors. Interestingly, in just about every form of cancer, while initially successful, cancer cells develop resistance to the inhibitors of EGFR following extended treatment (Sigismund et al., 2018). This resistance to EGFR-targeted therapies can be complex but in some cases can be due to (further) mutation in EGFR, upregulation of other RTKs or signalling receptors, or hyperactivation of down-stream 
signalling pathways that can operate independently of EGFR (Sigismund et al., 2018). Next, I briefly discuss some consequences of EGFR dysregulation in cancer.

\subsubsection{EGFR dysregulation in cancer}

EGFR gene disruption such as mutations or copy-number amplifications alters the normal signalling pathways, and have been reported in a number of cancers including NSCLC, glioblastoma, and forms of TNBC. The overexpression of EGFR increases the number of available receptors for ligand binding and dimerization (Chung et al., 2010; Sawano et al., 2002; Wilson et al., 2009; Wiley 1988). Consequently, the increase in number of activated receptors leads to the upregulation of the downstream signalling pathways (Chung et al., 2010; Sawano et al., 2002; Wilson et al., 2009; Wiley 1988). Mutations in EGFR may also disrupt the interaction with different proteins, important for its turnover or to inhibit receptor signalling. In some cancers, EGFR contains a mutation that perturbs the recruitment of E3 ligase, Casitas B-lineage lymphoma (Cbl), which is important for its downregulation and lysosomal degradation (Roskoski et al., 2014; Grandel et al., 2007; Han et al., 2007; Schmit et al., 2003). As a result, this type of EGFR mutation in certain cancers leads to prolonged signalling (Roskoski et al., 2014; Grandel et al., 2007; Han et al., 2007; Schmit et al., 2003). Furthermore, other mutations were identified in the extracellular domain of EGFR (e.g. EGFRvIII) (Grandal et al., 2007; Han et al., 2007; Schmit et al., 2003), allowing ligand independent activation of EGFR (Grandal et al., 2007; Han et al., 2007; Schmit et al., 2003). TKI, gefitinib, targets the mutated form of EGFR found in some lung cancers (Morgillo et al., 2016). However, drug resistance emerges as additional mutations appear in patients after extended treatment with gefitinib (Morgillo et al., 
2016). These examples demonstrate that disruptions in EGFR expression, such as overexpression/amplification or mutation, can alter its activity to potentiate signalling in certain types of cancer.

These alterations of EGFR that are common in many cancers that lead to both activation of oncogenic signalling and to drug resistance indicate two important considerations for EGFR signalling in cancer: 1) More information about the mechanisms that control EGFR signalling is needed, in order to develop therapies that avoid resistance to EGFR-targeted therapies and 2) targeting EGFR downstream signals that are common to not just EGFR but other signalling receptors and critical for cancer cell survival and growth, may reveal important alterative or combination drug targets. I will examine the former in chapter 3, examining how a novel mechanism of control to EGFR impacts receptor signalling outcome. I will also examine the latter, by studying a key signalling intermediate activated by EGFR and other receptors, mTORC1, in chapters 4 and 5. Akt, PLC $\gamma 1$, and Erk pathways described are signals regulated through the activation of EGFR. Akt signalling and to some extent, Erk signalling, converge on a key substrate, mTORC1, which is a master regulator protein, which I examine next.

\subsubsection{The discovery of mTOR}

Rapamycin, a macrolide compound, was first discovered on Easter island during the collection of soil samples in search of antimicrobial agents. This isolated soil sample was found to not only have antimicrobial activity but had interesting properties that inhibits tumor growth, fungal growth, and act as an immunosuppressive reagent (Eng et al., 1984; Martel et al., 1977; 
Vezina et al., 1975). After discovering rapamycin's impact on human cell physiology, it was later found to associate with a protein called 12-kDa FK506-binding protein (FKBP12) in cells, which was an important property to inhibit cell proliferation and growth (Chung et al., 1992). It was discovered that this inhibitory effect of rapamycin-FKBP12 complex converged on a protein later named mechanistic target of rapamycin or mTOR (Saber et al., 1995; Sabatini et al., 1994; Brown et al., 1994). Since this discovery, mTOR has now been implicated to control a wide variety of different cellular processes but also found to be dysregulated in many diseases. Importantly, mTOR is emerging as a master regulator of cell physiology relevant to cancer. I discuss the structure and properties of mTOR, how it is activated/regulated and in turn, the outcomes and functions of mTORC1 next.

\subsection{2 mTOR: two different complexes mTORC1 and mTORC2}

mTOR is a serine/threonine kinase that forms two distinct complexes, mTORC1 and mTORC2, each of which controls different cellular processes such as growth, survival, and proliferation (Saxton and Sabatini, 2017). mTORC1 contains three core proteins, mTOR, regulatory protein associated with mTOR (Raptor), and mammalian lethal with Sec13 protein 8 (mLST8) (Fig. 1.8) (Kim et al., 2003; Hara et al., 2002; Kim et al., 2002). Raptor mediates the recruitment of proteins to mTORC1 via a signalling motif called TOR signalling motif (TOS) (Nojima et al., 2003; Schalm et al., 2003). mLST8 associates with mTOR by interacting with its kinase domain to stabilize the kinase activation loop (Fig. 1.8) (Yang et al., 2013). mTORC1 also contains two other subunits that act as inhibitory proteins, DEP domain containing mTOR interacting protein (DEPTOR) (Peterson et al., 2009) and proline-rich Akt substrate of $40 \mathrm{kDa}$ 
(PRAS40) (Fig. 1.8) (Sancak et al., 2007; Vander Haar et al., 2007; Wang et al., 2007). Over the years, the mechanism by which rapamycin elicits an inhibitory effect on mTORC1 has been resolved. Following formation of a complex with FKBP12, rapamycin also binds the catalytic region of mTOR, preventing interaction with other substrates, thus preventing their activation (Yang et al., 2013).

mTORC2 is different from mTORC1, in that it is largely insensitive to rapamycin treatment. Similar to mTORC1, mTORC2 contains subunits of mTOR and mLST8, however in contrast to mTORC1, it contains subunits of rapamycin-insensitive companion of mTOR (Rictor) instead of Raptor (Jacinto et al., 2004; Sarbassov et al., 2004), Protor 1/2 (Pearce et al., 2007; Thedieck et al., 2007; Woo et al., 2007). In addition, mTORC2 also contains mSin1 subunit (Fig. 1.8) (Frias et al., 2006; Jacinto et al., 2006; Yang et al., 2006). Interestingly, while mTORC2 is insensitive to acute rapamycin treatment, long term treatment with rapamycin may perturb function of mTORC2, as rapamycin does bind to mTOR, new mTORC2 complexes may have difficulty forming (Lamming et al. 2012). As mTORC1 and mTORC2 are equipped with distinct subunits, it is not surprising that they also control different cellular physiology pathways, as they integrate different signals and have distinct substrates. mTORC1 activity is regulated by the integration of multiple pathways that include both mitogenic and metabolic signals, and mTORC1 will be the focus of much of my thesis and thus of this introduction, and examined next. 


\subsubsection{Regulation of mTORC1}

In broad terms, two modes of regulation have been described for mTORC1 activity, which are growth factor/RTK signalling (mitogenic) and metabolite availability signalling (metabolic). 1) During growth factor stimulation and activation of RTKs (as described in the EGFR section), Akt becomes activated allowing for the subsequent regulation of downstream targets (Manning and Cantley, 2007). One of the downstream targets of Akt is a GTPase activating protein (GAP), tuberous sclerosis protein 1/2 (TSC 1/2) (Inoki et al., 2003; Tee et al, 2003). Akt phosphorylates TSC2, impairing GAP activity towards GTPase protein Rheb (Fig. 1.9) (Long et al., 2005; Inoki et al., 2003; Tee et al, 2003). Inhibited TSC1/2, allows for Rheb to be GTP bound, which binds and activates mTORC1.

mTORC1 also requires metabolic signalling for its subsequent activation. Although extensive detailed information will be discussed on mTORC1's metabolic sensing ability later in this section, briefly, in nutrient replete conditions (amino acid abundance), the lysosomal VATPase will become activated (Jung et al., 2015; Rebsamen et al., 2015; Wang et al., 2015) leading to the activation of the Rag GTPase proteins (Fig. 1.9) (Bar-peled et al., 2012). Activated Rag proteins recruit mTORC1 to the lysosomal surface, where Rheb activates mTORC1 (Fig. 1.9) (Saxton and Sabatini, 2017; Efeyan et al., 2012; Sancak et al., 2010). As discussed below, mTORC1 not only senses amino acids but also senses other metabolites such as ATP (indirectly) via other signalling pathways involving AMPK, and lipids. I examine the metabolic sensors that converge to activate mTORC1 next. 


\subsubsection{Energy sensing by mTORC1: the role of AMPK}

Cell growth, proliferation, and protein translation are major processes that require high levels of energy expenditure and biomass accumulation. Controlling these processes requires regulation of the master regulator, mTORC1. When cellular energy in the form of ATP begins to be depleted, mTORC1 indirectly senses energy loss and becomes inhibited. mTORC1 senses changes or fluctuations in ATP, AMP, or ADP levels indirectly through AMPK (Herzig and Shaw, 2018). AMPK binds to ATP, AMP, or ADP, depending on the metabolic state of the cell, which will dedicate how it is activated (Gowan et al., 2013; Hardie et al., 2011). In energy stress, when AMP and ADP are abundant relative to ATP after extensive energy expenditure, AMPK is activated and regulates downstream targets, such as mTORC1 (Fig. 1.10) (Gowan et al., 2013; Hardie et al., 2011). It is also suggested that ischemia, hypoxia, and glucose deprivation activate AMPK (Qi and Young, 2015; Yun and Zierath, 2006). Furthermore, upstream substrate, liver kinase B1 (LKB1) also senses energy stress, positively regulating AMPK activity, therefore inhibiting mTORC1 (Shaw et al., 2004).

Two mechanisms have been proposed for the ability of AMPK to regulate mTORC1: 1) Activated AMPK phosphorylates TSC2, enhancing its GAP activity for Rheb and thus leading to inhibition of mTORC1 function (Fig. 1.10) (Inoki et al., 2003) 2) Activated AMPK phosphorylates and inhibits Raptor (subunit of mTORC1), blocking subsequent activity of mTORC1 (Fig. 1.10) (Shackelford et al., 2008). Thus, AMPK inhibits mTORC1, allowing coordination of ATP supply with the functions of mTORC1. 


\subsubsection{Lipid and amino acid sensors of mTORC1}

In addition to the indirect sensing of ATP levels via AMPK, mTORC1 also responds to the levels of certain lipids and amino acids. A study conducted by Castellano et al. in 2017, showed that in addition to the better understood regulation of mTORC1 by amino acids, mTORC1 activity is regulated through lipid sensing at the lysosome (Castellano et al., 2017). In the presence of intraluminal cholesterol within the lysosome, cholesterol binds and interacts with lysosomal amino acid transporter, sodium-coupled neutral amino acid transporter 9 (SLC38A9) (Castellano et al., 2017). Cholesterol binding to SLC38A9 mediates activation of Rag proteins, and subsequently promotes the recruitment and activation of mTORC1(Castellano et al., 2017).

mTORC1 senses amino acid availability through numerous mechanisms, sensing both intralysosomal and cytosolic pools of amino acids. Intralysosomal pools of amino acids are sensed by SLC38A9 specifically through arginine (Wang et al., 2015). In arginine fed cells, SLC38A9 activates the V-ATPase-Ragulator-Rag complex, which recruits and activates mTORC1 at the lysosome (Fig. 1.11) (Jung et al., 2015; Rebsamen et al., 2015; Wang et al., 2015). Cytosolic pools of amino acids also enhance mTORC1 activity, which occurs as a result of numerous signals impacting the GAP activity towards Rag 1/2 complex (GATOR 1/2). GATOR1 contains three subunits, DEP Domain Containing 5 (DEPDC5), NPR2 like, GATOR1 complex subunit (NPRL2), and NPR3 like, GATOR1 complex subunit (NPRL3), which acts as a GAP for Rag GTPases (Shen et al., 2019), acting as a negative regulator for Rag (Fig. 1.11) (Bar-Peled et al., 2013). In the absence of amino acids (and thus GATOR1 is active), the GAP activity of GATOR1 negatively regulates Rag GTPase, impairing mTORC1 recruitment and activation to the lysosome. GATOR2 directly contacts amino acid sensors and act as a positive 
regulator for mTORC1 activity by inhibiting GATOR1 activity (Bar-Peled et al., 2013). Overall, this mechanism leads to mTORC1 activation during amino acid sufficiency, further detail will be

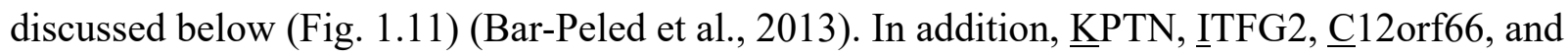

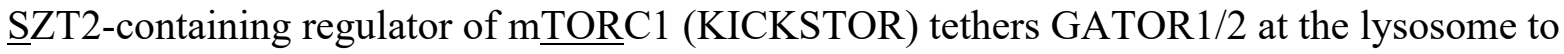
regulate Rag GTPase activity (Fig. 1.11) (Wolfson et al., 2017). Given this architecture of GATOR1/2 suppression of Rag GTPases and thus control of mTORC1 activation, several recent mechanisms of direct protein amino acid sensors have been identified that allow modulation of this system to impact mTORC1, including Sestrin2, Castor, S-adenosylmethionine sensor upstream of mTORC1 (SAMTOR), and Folliculin-FNIP2, as discussed next.

Sestrin 2 and Castor 1 are cytosolic amino acid sensors that regulate mTORC1 indirectly. Specifically, Sestrin2 (Chantranupong et al., 2014; Parmigiani et al., 2014) and Castor1 (Chantranupong et al., 2016; Saxton et al., 2016) bind to leucine and arginine, respectively. This interaction inhibits the activity of Sestrin2 and Castor1, inhibiting GATOR1, followed by activation of mTORC1 in a Rag GTPase dependent manner (Fig. 1.11) (Chantranupong et al., 2016; Saxton et al., 2016; Chantranupong et al., 2014; Parmigiani et al., 2014). In arginine and leucine deprived conditions, Sestrin2 and Castor1 inhibit GATOR 2, leading to GATOR 1 dependent mTORC1 inhibition (Fig. 1.11) (Chantranupong et al., 2016; Saxton et al., 2016; Chantranupong et al., 2014; Parmigiani et al., 2014).

By a mechanism distinct from Sestrin2 and Castor1 amino acid dependent mTORC1 activity, SAMTOR senses cytosolic methionine but directly interacts with GATOR1 and not GATOR2 (Fig. 1.11) (Gu et al., 2017). In methionine deprived conditions, SAMTOR interacts 
and binds to GATOR1, activating its GAP activity, inhibiting Rag GTPases, leading to inhibition of mTORC1 (Fig. 1.11) (Gu et al., 2017). When methionine is replenished with in the cell, methionine binds to SAMTOR, inhibiting its activity, leading to suppression of GATOR1 GAP activity, allowing for Rag GDP to exchange for GTP and enhancement mTORC1 activation (Gu et al., 2017).

Another amino acid sensing mechanism different from the three highlighted above, Folliculin-FNIP2 protein complexes sense amino acids independent of signalling towards GATOR 1/2 complex and directly regulates Rag proteins (Fig. 1.11) (Petit et al., 2013; Tsun et al., 2013). In the presence of amino acids (specific amino acids have yet to be elucidated), Folliculin-FNIP2 will activate Rag proteins for the subsequent activation and recruitment of mTORC1 to the lysosome (Fig. 1.11) (Petit et al., 2013; Tsun et al., 2013). This demonstrates that the regulation of $\mathrm{mTORC} 1$ via different metabolites is a complex process that involves indirect sensors of both lipids and amino acids. Depending on the presence of specific lipids and amino acids, it will lead to changes in mTORC1 activation.

\subsubsection{Regulation of mTORC1: signalling output}

mTORC1's complex and extensive regulation modulates its function, leading to changes in many cell physiological events such as mRNA translation, metabolism, transcription regulation, and protein turnover. In control of mRNA translation, mTORC1 phosphorylates and promotes the activity of a critical protein, p70S6 kinase (S6K1) (Holz et al., 2005). Activating S6K1 leads to protein synthesis via interaction eukaryotic initiation factor 4F (eIF4F) complex, important for mRNA translation efficiency (Fig. 1.12) (Holz et al., 2005). In addition to the 
regulation of $\mathrm{mTORC1}$ via metabolism, $\mathrm{mTORC} 1$ also contributes to the reciprocal regulation of metabolism by promoting transcription of genes that are involved in lipid synthesis and glucose metabolism. For example, mTORC1 interacts and activates transcription factor sterol responsive element binding protein (SREBP), promoting the expression of genes that are required for lipid synthesis (Fig. 1.12) (Porstmann et al., 2008). mTORC1 also regulates glucose metabolism by increasing the translation of the transcription factor HIF1 $\alpha$ (Fig. 1.12) (Duvel et al., 2010), which increases the expression of glycolysis pathway enzyme phospho-fructo kinase (PFK) and glucose transporter1 (GLUT1) (Fig. 1.12) (Duvel et al., 2010). As such, mTORC1 is an important contributor to metabolism, as it is controls the expression of important metabolic regulators for the replenishment of metabolites within the cell.

mTORC1 is also a key regulator of protein turnover, as a result of mTORC1 being a critical gatekeeper of a catabolic processed called macroautophagy (henceforth autophagy). Autophagy is defined as the degradation of internal components (which may contain biomolecules such as proteins and lipids, and in some cases entire organelles), in which components are encased in a double membrane (autophagosome) packaged by many accessory proteins, leading to subsequent fusion with the lysosome and cargo degradation (Yu et al., 2018). There are different types of autophagy, grouped according to the cargoes that are degraded/targeted (Yu et al., 2018). The process of autophagy involves sequential steps, including nucleation, elongation, maturation, fusion, and degradation (Glick et al., 2010). Briefly, autophagy is initiated by the activation of Unc-51 like autophagy activating kinase (ULK) complex, containing ULK1/2, autophagy related protein 13 (Atg13), Atg101, and focal adhesion kinase family interacting protein of $200 \mathrm{kD}$ (FIP200) (Jung et al., 2009). After 
activation of the ULK complex, it activates class III PI3K complex, which contains class III PI3K, protein 150 (p150), Beclin-1, and Beclin-1-associated autophagy-related key regulator (barkor), to nucleate and assemble the phagophore membrane (Jaber et al., 2012; Sun et al., 2009; Chen et al., 2008). During the elongation and maturation phase, Atg3, Atg4, Atg5, Atg7, Atg10, Atg12, and Atg like protein $16(\operatorname{Atg} 16 \mathrm{~L})$, coordinate a complex and multistep process to convert and bind microtubule-associated protein 1 light-chain 3 - II (LC3 - II) to the initial phagophore (Romanov et al., 2012; Yamaguchi et al., 2012; Satoo et al., 2009; Tanida et al., 2004; Mizushima et al., 2003). LC3 - II mediates the expansion and closing of phagophores (now autophagosome) forming the phagosome in autophagy (Kabeya et al., 2000). Next, autophagosome containing cargo is trafficked and fused with the lysosome, forming the autolysosome, resulting in the degradation of cargo via lysosomal enzymes (Webb et al., 2004). Degraded material become monomeric units (e.g. Amino acids), which are exported via lysosomal transporters and permeases toward the cytosol (Wu et al., 2016; Mizushima 2007).

There are different mechanisms to trigger the autophagy machinery. Briefly, autophagy is regulated by the following: 1) In nutrient replenished cells, mTORC1 phosphorylates and inhibits ULK1/2, which is a protein responsible for triggering autophagy (Fig. 1.12) (Yu et al., 2018; Kim et al., 2011). In nutrient-deprived cells, mTORC1 is largely suppressed, which in turn leads to activation of ULK1/2, recruiting the autophagy machinery (Yu et al., 2018; Kim et al., 2011) 2) Other studies have also highlighted that mTORC1 interacts with lysosomal biogenesis and autophagy protein called transcription factor EB (TFEB) (Martina et al., 2012; RoczniakFerguson et al., 2012; Settembre et al., 2012). In the presence of nutrients, mTORC1 phosphorylates TFEB and prevents its movement into the nucleus (Martina et al., 2012; 
Roczniak-Ferguson et al., 2012; Settembre et al., 2012). In nutrient deprived conditions, mTORC1 is inactivated, promoting the translocation of TFEB into the nucleus, increasing gene expression of lysosomal and autophagy proteins (Martina et al., 2012; Roczniak-Ferguson et al., 2012; Settembre et al., 2012). Thus, mechanisms for inhibiting autophagy requires the activity of mTORC1. Furthermore, mTORC1 is important for regulating metabolism, to balance processes under fluctuating conditions of energy and biomolecule availability and demand. In addition to the dual action of metabolism for regulating mTORC1's activity and signals that are delivered out for metabolic processes, mitogenic signals also regulate mTORC1 activity, including signals from mTORC2.

\subsubsection{Regulation of mTORC2}

While mTORC1 is a sensor and regulator of metabolism, mTORC2 is mostly controlled by mitogenic signalling emanating from signalling receptors and has been mostly implicated in controlling survival, proliferation, and migration. mTORC2 promotes growth and survival signals by contributing to the activation of Akt, as described above (Fig. 1.13) (Sarbassov et al., 2005). The mTORC2 component, mSIN1, serves to inhibit mTORC2 kinase activity in unstimulated cells (Liu et al., 2015). As a result of PI3K activation and generation of PI $(3,4,5)$ $\mathrm{P}_{3}$, mSIN1 binds to PI $(3,4,5) \mathrm{P}_{3}$, causing the release of its suppressive activity towards mTORC2, leading to the activation of $\mathrm{mTORC} 2$ for subsequent phosphorylation and activation of Akt (Fig. 1.13) (Liu et al., 2015). In addition to the regulation of Akt activation, mTORC2 also plays a role in the remodeling of actin cytoskeleton (Angliker and Rüegg 2013; Larsson 2006; McLaughlin et al., 2002). mTORC2 phosphorylates and activates PKC (Angliker and 
Rüegg 2013; Larsson 2006; McLaughlin et al., 2002). Activation of PKC leads to the phosphorylation of GAP-43-like proteins, myristoylated alanine-rich C-kinase, and GAP-43, which dissociates from $\mathrm{PI}(4,5) \mathrm{P}_{2}$ at the plasma membrane, promoting actin polymerization and extension (Angliker and Rüegg 2013; Larsson 2006; McLaughlin et al., 2002). These mechanisms of mTORC2-dependent regulation of pro-survival/pro-growth and cytoskeleton pathways signifies the distinct role of mTORC2 from mTORC1 in sensing and the regulation of metabolic activities.

The complex regulation of mTORC1 thus involves the integration of both mitogenic and metabolic signals. Emerging evidence indicates that in order for both mitogenic and metabolic signals to integrate and converge onto mTORC1, signals must be spatially organized, as receptors are organized at the plasma membrane and amino acid signalling is organized at lysosome. Thus, to spatially organize signals and ensure effective control of mTORC1 activity, the regulation of membrane traffic is required as it dynamically moves membranes to control protein activity.

\subsubsection{Membrane traffic properties}

Membrane traffic is defined as the movement of membrane materials to and from specific membrane organelle compartments, as a result of dynamic fusion and scission of membranes (Fig. 1.14) (Kaksonen and Roux, 2018; Knorr et al., 2017; Miaczynska 2013; Tokarev et al., 2009). Membrane traffic plays an important role for controlling many cellular events such as signalling, organelle organization, metabolism, and organization of cargo (Doherty and 
McMahon, 2009). There are many membrane traffic events that mediate exchange of materials between diverse compartments. The membrane traffic stage that specifically begins at the plasma membrane is called endocytosis. Endocytosis is defined as the internalization of membranebound cargo and/or membranous material from the cell surface, resulting in the formation of an intracellular vesicle. Endocytosis can occur by several distinct mechanisms, and each of these relies on different protein machinery (Fig. 1.14) (Miaczynska and Stenmark, 2008). Cargo (which is a term used to describe material including extracellular material, receptors, transporters and other proteins that are subject to endocytosis) at the plasma membrane are sequestered into a small concentrated membrane area, and are internalized with the aid of distinct molecular machineries that deliver cargoes to cells (Fig. 1.14) (Ferreira and Boucrot, 2018; Kaksonen and Roux, 2018; Mettlen et al., 2018; Doherty and McMahon, 2009; Freeman and Grinstein, 2014). These endocytic mechanisms are critical for the regulation of the activity of many proteins, as many cell surface proteins including signalling receptors, nutrient transporters and channels and adhesion proteins require access to their substrates and ligands that are found in the extracellular space. Hence, endocytosis mechanisms are critical regulators of the cell surface proteome. There are different classes of internalization which include, clathrin mediated endocytosis (CME), and clathrin independent endocytosis (CIE) a term used to describe a collection of mechanisms that includes caveolae endocytosis, macropinocytosis, and phagocytosis, and all differ in terms of the protein machinery used in their specific processes, which will be discussed next. 


\subsubsection{Classes of Endocytosis}

CME is the principal method for internalization of receptor-bound macromolecules from the cell surface. CME is a complex multistep process involving the coordination of the time and space of many proteins for the internalization of receptor-bound macromolecules (Fig. 1.15) (Kaksonen and Roux, 2018; Mettlen et al., 2013; Cocucci et al., 2012; McMahon and Boucrot, 2011; Taylor et al., 2011; Schmid and McMahon, 2007). CME begins with ligand bound receptors recruited into small invaginations on the plasma membrane called clathrin coated pits (CCP) (Fig. 1.14 and Fig 1.15) (Kaksonen and Roux, 2018; Mettlen et al., 2013; Cocucci et al., 2012; McMahon and Boucrot, 2011; Taylor et al., 2011; Schmid and McMahon, 2007). These CCPs are composed of critical molecular components that aid in the formation of CCPs including, PI45 $\mathrm{P}_{2}$, adaptor protein 2 (AP2), clathrin, and dynamin 1/2 (Kaksonen and Roux, 2018; Mettlen et al., 2013; Cocucci et al., 2012; McMahon and Boucrot, 2011; Taylor et al., 2011; Schmid and McMahon, 2007). AP2 is a heterotetrameric protein that contains subunits that coordinate the recruitment of proteins to induce $\mathrm{CCP}$ formation: the $\alpha$ subunit binds to $\mathrm{PI}_{45} \mathrm{P}_{2}$ at the plasma membrane, $\mu 2$ subunit binds to cargo contain protein sequence $\mathrm{Yxx} \phi$ or tyrosine any 2 amino acids - hydrophobic, and the $\beta$ subunit binds and recruits clathrin (Boucrot et al., 2010). Through the coordination of AP2 and subsequent recruitment of clathrin to CCPs, the plasma membrane undergoes inward invagination towards the interior of the cell (Kaksonen and Roux, 2018; Mettlen et al., 2013; Cocucci et al., 2012; McMahon and Boucrot, 2011; Taylor et al., 2011; Schmid and McMahon, 2007). In addition to clathrin, AP2 and dynamin, a number of membrane sculpting proteins, such as eps15 interaction protein (epsin) (Sen et al., 2012) and 
endophilin, are recruited to the site of CCVs formation to sculpt the flat membrane into growing vesicles.

The next step of CME is the scission step of the neck of the CCP, which is mediated by GTPase protein dynamin $1 / 2$, leading to a free clathrin coated vesicle that is subsequently released from the plasma membrane into the cytoplasm (Kaksonen and Roux, 2018; Mettlen et al., 2013; Cocucci et al., 2012; McMahon and Boucrot, 2011; Taylor et al., 2011; Schmid and McMahon, 2007). One of the final steps of CME uses the protein synaptojanin, which hydrolyze $\mathrm{PI}_{45} \mathrm{P}_{2}$, leading to the release of the clathrin coat, ending with a cargo bound vesicle and trafficking to a downstream endomembrane compartment, the early endosomes (Kaksonen and Roux, 2018; Mettlen et al., 2013; Cocucci et al., 2012; McMahon and Boucrot, 2011; Taylor et al., 2011; Schmid and McMahon, 2007). Examples of cargo from CME that are internalized toward the cell include, protein transmembrane receptors and their specific ligand (Linton and Bonifacino, 2013), such as EGFR (Goh and Sorkin, 2013, Sorkin and Goh, 2009) and Transferrin receptor (Liu et al., 2010).

Given that EGFR is a signalling receptor that is recruited to CCPs and subjected to internalization concomitantly to initiation of receptor signalling, it is possible that the signalling by EGFR and the recruitment to CCPs and thus clathrin are functionally linked and interdependent. Currently, it is not well understood how CME events such as CCP initiation (requiring the protein clathrin to form a molecular scaffold at the membrane) or cargo endocytosis (requiring scission from the plasma membrane mediated by dynamin 1/2), may play an important role in signalling. Dynamin1 was thought to be brain specific but it is expressed in 
all tissues (Srinivasan et al., 2018; Chen et al., 2017; Reis et al., 2017; Reis et al., 2015) Furthermore, dynamin1 is not merely redundant with dyanmin2, and dynamin1 appears to selectively regulate the internalization of specific receptors (Reis et al., 2017) and be subject to regulation by specific factors like p53 (Lakoduk et al., 2019). The question emerges if dynamin1 contributes to EGFR signalling and endocytosis, which I will examine in chapter 3.

In contrast to $\mathrm{CME}, \mathrm{CIE}$ pathways utilize distinct molecular machineries for the formation of vesicles and internalization of cargoes (Ferreira and Boucrot, 2018). Similarly, a specific class of CIE, Calveolae-dependent endocytosis, does not use clathrin but uses caveolin to internalize cargo such as low-density lipoprotein (LDL) receptor (Fig. 1.15), although, the evidence from studies of caveolin internalization is complex and mechanisms are still not well defined (Ferreira and Boucrot, 2018; Sandvig et al., 2018). Another form of CIE, macropinocytosis, is the internalization of non-selective cargo by taking large "gulps" of extracellular material through generation of actin-dependent membrane ruffles (Fig. 1.15) (Fairn and Grinstein, 2012; Lim and Gleeson, 2011). Given that the work in this thesis will focus on clathrin-mediated endocytosis and the role of clathrin in modulating signalling by EGFR, I will not discuss in detail various forms of CIE, although the possible contribution of these various endocytic mechanisms to Akt signalling was recently reviewed (Sugiyama et al., 2019).

Phagocytosis, a different internalization process from CME and CIE, is receptormediated and actin cytoskeleton-dependent form of endocytosis that is performed by professional phagocytosis cells (termed phagocytes) (Fig. 1.15) (Rosales and Uribe-Querol, 2017). Phagocytosis differs from endocytosis, as this phenomenon allows the receptor-mediated 
internalization of larger particles and cargo engulfed include bacteria, foreign material, and dead cells (Fig. 1.15) (Rosales and Uribe-Querol, 2017). This differs from the other endocytic events as evidence shows that the phagosome may fuse directly with the lysosome, forming the phagolysosome, which eventually degrades the cargo (Fig. 1.15) (Levi et al., 2016; Fairn and Grinstein, 2012; Luzio et al., 2007). All endocytic events end with cargo compartmentalized into membrane vesicles, which are then organized, sorted, and trafficked to other endomembrane compartments through the endocytic pathway. I will not discuss phagocytosis in detail as this thesis will focus on mechanisms related to CME.

\subsubsection{Endocytic membrane traffic after internalization}

After remodelling the plasma membrane using specific endocytosis machinery, a vesicle is generated, trafficked and fused with the early endosome (Naslavsky and Caplan, 2018; Jovic et al., 2010; Diaz et al., 1988). Early endosomes are important endomembrane compartments, as

they are classified as "sorting organelles," which sorts and delivers received cargo from multiple endocytic compartments to other compartments (Naslavsky and Caplan, 2018; Jovic et al., 2010;

Diaz et al., 1988). The early endosome is marked by several different proteins, each with specific functions, which includes: Ras-related protein 5 (Rab5), Rab4, and early endosome antigen 1 (EEA1). Rab5 is the canonical early endosome Rab GTPase, and is essential for the recruitment of EEA1 and for the homotypic vesicle fusion (or heterotypic fusion of vesicles with endosomes) (Langemeter et al., 2018; Sönnichsen et al., 2000; Gorvel et al., 1991; Stenmark et al., 1998) Rab4 is involved in rapid recycling of cargoes back to the plasma membrane (Van Der Sluijs et al., 1991; Sönnichsen et al., 2000; Langemeyer et al., 2018). EEA1, a Rab5 effector protein, 
contains a cysteine-rich motif (FYVE), which binds to phosphatidylinositol-3-phosphate (PI3P) via C-terminus, and binds to GTP-bound Rab5 via N-terminus (Murray et al., 2016). The structure of EEA1 is important for endosomal tethering and mediating endosomal fusion (Murray et al., 2016; Zerial et al., 1999).

Interestingly, there are a distinct subset of early endosomes demarked by APPL1 through which some specific internalized cargo traverse en route to the so-called "classical" EEA1positive early endosome. APPL1 endosomes are thought to be important for the regulation of signalling events (Diggins and Webb, 2018). APPL1 endosomes are described as the "early early endosomes" that is found in between the plasma membrane and EEA1 early endosomes, in the endocytic pathway (Diggins and Webb, 2018). Schenck et al. in 2008 demonstrated that GSK3 $\beta$ is regulated by the recruitment of Akt to APPL1 early endosomes, resulting in the phosphorylation-dependent inhibition of GSK3 $\beta$ within this membrane compartment (Schenck et al., 2008). Furthermore, this unique signalling platform is an important mechanism that controls cell survival (Schenck et al., 2008). Recently, Reis et al demonstrated that APPL1 early endosomes are required to suppress GSK3 $\beta$, and this in turn results in the regulation of dynamin1, and internalization of specific cargoes via CME (Reis et al., 2015). Hence, early endosomes are heterogenous, yet some types are important endomembranes for both the organization of cargo and signalling platforms for different cellular functions.

Some early endosomes undergo a maturation phase, involving a switch from early endosome to late endosome identities (Balderhaar et al., 2013; Poteryaev et al., 2010). This process is driven by conversion of Rab5-positive compartments to compartments containing 
Rab7. In this phenomenon, early endosomal Rab5 is inactivated by the Sand1/Mon1/CCZ1 complex (Balderhaar et al., 2013; Poteryaevet al., 2010). Subsequently, Sand1/Mon1/CCZ1 complex recruits the homotypic fusion and protein sorting (HOPS) complex, which in turn recruits and activates Rab7 (Balderhaar et al., 2013; Poteryaevet al., 2010). Recruitment and activation of Rab7 promotes the full maturation of early endosomes to the late endosome/multivesicular bodies (MVB) (Balderhaar et al., 2013; Poteryaevet al., 2010). The late endosome/MVB containing cargo are then trafficked and fused to the lysosomes, and thus fusion of late endosomes and lysosomes is also mediated by the HOPS complex (Bajaj et al., 2018; Balderhaar et al., 2013; Poteryaevet al., 2010).

The lysosome not only plays an important and well-known role in macromolecule degradation and processing, but it is also increasingly appreciated as an important signalling platform. To ensure the former degradative capacity of the lysosome, the V-ATPase is a lysosome membrane protein that is responsible for acidifying the lysosome (Pamarthy et al., 2018). This allows for the activation of degradative enzymes including proteases, nucleases, and lipases, to target cargo trafficked towards the lysosome (Pamarthy et al., 2018; Platt et al., 2018).

The lysosome also acts as a platform for a number of different cellular signals and cues. The Ragulator/Rag complex, which is regulated by metabolic pathways, is required for the activation and recruitment of mTORC1 to the lysosome (Panic et al., 2011; Saxton and Sabatini, 2017). Phosphorylated mTORC1 sequesters and inhibits TFEB at the lysosome (Bajaj et al., 2018). In contrast, when mTORC1 is inhibited, TFEB undergoes dephosphorylation and subsequent translocation from the lysosome to the nucleus to activate lysosomal biogenesis 
through regulating gene expression (Bajaj et al., 2018). These examples demonstrate the importance of lysosomes acting as multitasking organelles for regulating the degradation of cargo as well as acting as an organizational platform for signalling pathways. Endomembrane traffic is an important cellular mechanism that spatially and temporally organizes signals, that in turn regulates different cell processes. Endomembrane traffic also allows for transport of cargoes from distinct membrane compartments to lysosomes, which allows for the organization of the lysosome, which may be especially important for mTORC1 signalling (Apodaca and Brown, 2014). However, how various membrane traffic phenomena regulates the organization of the lysosome to in turn affect specific mTORC1 signals is not fully understood.

mTORC1 is indirectly regulated by an upstream signals from Akt, which interacts with a variety of different substrates. One important substrate regulated by the PI3K-Akt pathway is GSK3. Notably, several lines of evidence suggest that GSK3 may be regulated by more than the canonical phosphorylation by Akt. I will examine the regulation of GSK3 and mTORC1 in Chapters 3 and 4, and as such here discuss the regulation, localization, and contribution to different diseases of GSK3.

\section{$\underline{1.6 .1 \mathrm{GSK} 3 \text { and properties }}$}

Originally thought to have only function in glycogen metabolism and thus named, glycogen synthase kinase 3 (GSK3) has been revealed to function in many different processes such as cell growth, apoptosis, stem cell self renewal, and many others (Cormier and Woodgett, 2017; Mancinelli et al., 2017; Beurel et al., 2015; Medina and Wandosell, 2011). GSK3 is a 
serine/threonine kinase and contains two different closely related but not completely redundant paralogs: GSK3 $\alpha$ and GSK3 $\beta$ (Beurel et al., 2015; Medina and Wandosell, 2011; Woodgett 1990). GSK3 $\alpha$ and GSK3 $\beta$ are largely identical except for some key differences including the amino acid composition of the protein sequence at specific regions of the proteins: GSK3 $\alpha$ contains a glycine rich region near the N-terminus missing in GSK3 $\beta$ and the location of different phosphorylation residues varies between these paralogues (Fig. 1.16) (KaidanovichBeilin and Woodgett, 2011).

GSK3 was first discovered in 1980 by Philip Cohen's lab, as one of the effectors to negatively regulate glycogen synthase by phosphorylation and thus inhibiting glycogen metabolism (Rylatt et al., 1980). Shortly after this discovery, in 1983 insulin stimulation was found to lead to the activation of glycogen synthase, which resulted in dephosphorylation of glycogen synthase at the residues that are substrates of GSK3, which suggested that insulin signalling may inhibit GSK3 activity (Parker et al., 1983). Since then, Akt was discovered to induce GSK3 phosphorylation and inactivation during insulin signalling (Cross et al., 1995). Over the years, many signalling proteins have been identified to control GSK3 activity (Beurel et al., 2015). In addition, a wide range of substrates beyond glycogen synthase has expanded the capabilities of GSK3 well beyond control of glycogen metabolism, as will be discussed below. Furthermore, many studies have also observed and identified roles for GSK3 in many diseases including diabetes, Alzheimer's disease and cancer, which will also be discussed later in this section. The regulation and activity of GSK3 is complex and context-specific, and subject to modulation by post-translational modification, control of subcellular localization, regulation of protein-protein interactions and control of recognition downstream substrates. Thus, to 
understand how GSK3 activity is regulated, discussed below are mechanisms by which different upstream effectors modify GSK3, altering its subsequent activity.

\subsubsection{The regulation of GSK3 activity and localization}

Different modifications of GSK3 may alter both its activity and localization. Many mechanisms have been identified that result in post-translational modification of GSK3 including phosphorylation, citrinullation, and calpain cleavage, each of which may control GSK3 activity and localization (Cormier and Woodgett, 2017; Mancinelli et al. 2017; Beurel et al., 2015; Medina and Wandosell, 2011; Jope and Johnson, 2004). Each of these modifications and regulatory mechanisms will be discussed below.

GSK3 is part of a rare subset of proteins which exhibit enzymatic activity that is constitutively active in resting cells, until key signalling events are activated to inactivate or otherwise regulate GSK3 (Cormier and Woodgett, 2017). The main mechanism that has been thoroughly studied for GSK3 regulation is phosphorylation, in which the specific phosphorylation of serine 21 on GSK3 $\alpha$ or corresponding Serine 9 on GSK3 $\beta$ inhibit certain aspects of GSK3 kinase activity. This phosphorylation of GSK3 is mediated by a number of kinases such Akt, PKA, PKC, p90RSK, and S6K (Fig. 1.16) (Cormier and Woodgett, 2017; Beurel et al., 2015; Frame et al., 2001; Cross et al., 1995). Mechanistically, when the S21/9 residue is phosphorylated, this residue is part of a motif that acts as a pseudosubstrate that occupies the substrate recognition domain of GSK3, hindering interaction with other downstream substrates (Frame et al., 2001). A number of other phosphorylation sites serve different purposes 
for GSK3 activity, such as Y279 on GSK3 $\alpha$ and Y216 on GSK3 $\beta$ (Fig. 1.16) (Frame and Cohen, 2001; Huges et al., 1993). It is proposed that phosphorylation of Y279 and Y216 are important for maximum kinase activity by GSK3 $\alpha$ and GSK3 $\beta$, respectively (Cole et al., 2004). It has also been shown that Src-family kinases such as Src and Fyn, phosphorylates Y216 specifically on GSK3 $\beta$ to enhance its kinase activity, (Fig. 1.16) (Goc et al., 2014; Lesort et al., 1999). Furthermore, phosphorylation of GSK3 $\beta$ at T43 by Erk1/2 (Ding et al., 2005), S389 and T390 by p38 MAPK (Thorton et al., 2008) enhances the ability for S9 to become a good substrate for phosphorylation (Fig. 1.16). Major mitogenic signals does indeed lead to partial reduction of GSK3 activity but this is incomplete, other mechanisms of regulation of GSK3 are likely to exist.

\subsubsection{GSK3 subcellular localization}

Given that GSK3 is constitutively active, mechanisms other than post-translational modification are likely to contribute to the control of GSK3 function. The control of GSK3 localization may be important for the regulation of different physiological outcomes. The localization of GSK3, defines a subset of proteins and molecules with which it will interact, thus limiting both upstream regulatory processes that can impact GSK3 and access different substrates. The following are endomembrane compartments where GSK3 has been reported to be localized:

Plasma membrane: In the basal state, a complex called the destruction complex, which is an assembly of proteins, including adenomatous polyposis coli (APC), axis inhibition protein (Axin), casein kinase 1 (CK1), and $\beta$-catenin localize within the cytosol (Wu and Pan, 2010; Zeng et al., 2008). The destruction complex concentrates these proteins, allowing GSK3 to 
phosphorylate and promote the degradation of $\beta$-catenin (Wu and Pan, 2010; Zeng et al., 2008).

During Wnt signalling, the ligand Wnt binds GPCR Frizzled and co-receptor lipoprotein receptor-related protein (LRP) at the cell surface (Fig. 1.17) (Wu and Pan, 2010; Zeng et al., 2008). Furthermore, frizzled/LRP recruit proteins such as GSK3, Axis inhibition protein (Axin), CK1, and dishevelled (DSH) to the plasma membrane (Wu and Pan, 2010; Zeng et al., 2008). GSK3 phosphorylates a critical Serine or Threonine residue found within a motif PPP(S/T)P of LRP, that when phosphorylated occupies the phospho-binding pocket of GSK3 inhibiting kinase activity (Wu and Pan, 2010; Zeng et al., 2008; Zeng et al., 2005).

Early endosomes: GSK3 has been reported to localize to early endosomes, first by Schenck et al in 2008, who observed GSK3 $\beta$ to localize to APPL1 early endosomes (Schenck et al., 2008). APPL1 recruits both GSK3 $\beta$ and Akt to APPL1 early endosomes, allowing Akt to phosphorylate and inhibit GSK3 $\beta$ (Scheck et al., 2008). It was also reported by Reis et al. in 2015, that active GSK3 $\beta$ also negatively regulates dynamin1 dependent CME (Fig. 1.17) (Reis et al., 2015). Furthermore, when GSK3 3 is inhibited (mediated by its localization to Akt-APPL1 early endosomes), activated dynamin1 increases the initiation of CCP formation (Reis et al., 2015).

Mitochondria: GSK3 localizes to the mitochondria regulating aspects of metabolism. A report from Hoshi et al. in 1996 found that GSK3 $\beta$ localized at the mitochondria regulating pyruvate dehydrogenase activity (Hoshi et al., 1996). In hippocampal neuronal cultures, $\beta$-amyloid treatment, promoted activity of GSK3 $\beta$ leading to the phosphorylation and inactivation of pyruvate dehydrogenase (Hoshi et al., 1996). Furthermore, this lead to the decrease in production 
of acetyl-CoA, impairing acetylcholine production in cholinergic neurons (Fig. 1.17) (Hoshi et al., 1996).

Lysosome: It has also been reported that GSK3 also localizes to the lysosome (Fig. 1.17) to regulate lysosomal biogenesis and acidification (Liu et al., 2016; Azoulay-Alfaguter et al., 2015). According to a study Liu et al. in 2016, GSK3 $\beta$ localized to the lysosome interacting with a lysosomal protein, TFEB (Liu et al., 2016). GSK3 $\beta$ phosphorylates TFEB preventing it from translocating to the nucleus to trigger lysosomal biogenesis (Liu et al., 2016). In addition, evidence from Azoulay-Alfaguter et al. in 2015 showed that GSK3 $\beta$ controls lysosomal acidification (Azoulay-Alfaguter et al., 2015). Specifically, they showed the GSK3 $\beta$ enhances mTORC1 activity, decreasing lysosomal acidification (Azoulay-Alfaguter et al., 2015). This indicates that GSK3 $\beta$ can regulate mTORC1, thus affecting lysosomal acidification, but that the possible reciprocal regulation of GSK3 $\beta$ by mTORC1 (e.g. at the lysosome) has not been examine, and this will be the focus of my work in chapters 4 and 5 .

Nucleus: The localization of GSK3 to specific membrane compartments is not static, as GSK3 can be translocated to different membrane compartments depending on the different signals it receives. Two post translational modifications, citrunillation of GSK3 $\beta$ (Stadler et al., 2013) and calpain cleavage of GSK3 (Goñi-Oliver et al., 2007), both promote GSK3 translocation from the cytosolic to the nucleus (Fig. 1.17). During different cellular events, heat shock (Bijur and Jope, 2001), PI3K-Akt suppression in embryonic stem cells (Dalton and Bechard, 2009), in S phase of the cell cycle (Diehl et al., 1998), and engagement of cell senescence (Zmijewski and Jope, 
2004), GSK3 undergoes nuclear translocation, suggesting a regulated alteration in the function of GSK3 under these conditions.

All examples demonstrate that GSK3 dynamically localizes to different cellular compartments, and that this localization can be controlled by specific cues and signals, thus modulating how GSK3 controls different aspects of cellular physiology under specific conditions. For GSK3 to translocate to specific compartments within the cell, for example the nucleus, GSK3 requires both sorting motifs that allow GSK3 to access each compartment in a specific manner and regulation of this sorting that allows GSK3 to localize to the compartments in a context-specific manner. The former is partly understood, where as the regulation of sorting is still in its infancy, both of which I will examine in chapter 4 and 5.

\section{$\underline{\text { 1.6.4 GSK3} 3 \beta \text { nuclear localization and NLS }}$}

GSK3 $\beta$ contains non-canonical nuclear localization sequences (NLS) important for its nuclear translocation. Meares and Jope in 2007, identified two NLS sequences with in GSK3 $\beta$ : 1) a basic stretch of amino acids from residues 85-105 and 2) 9 amino acids located at the nterminus (residues 1 - 9 on GSK3 $\beta$ ), that mediates nuclear localization (Meares and Jope, 2007). Mutations in the basic stretch (85-105) or truncation to the first 9 amino acids of GSK3 $\beta$ prevented translocation into the nucleus (Meares and Jope, 2007). Although both sequences do not follow the regular NLS canonical sequence, perturbations of either these sequences prevented nuclear localization of GSK3 $\beta$. Notably, mutations of specific residues within the 85105 domain (R96A, R102G, and K103A) also impaired GSK3 $\beta$ kinase activity making 
understanding the contribution of physiological control of GSK3 $\beta$ nuclear localization separate from regulation of kinase activity challenging (Meares and Jope, 2007). Hence, while these two NLS sequences are both required for GSK3 $\beta$ nuclear localization, yet it is not well understood how signalling impacts its localization and function.

\subsubsection{GSK3 Substrate specificity}

The major mechanism for recognizing substrates by GSK3 is through a primed phosphorylation (Dajani et al., 2001; Ter Haar et al., 2001). Substrates subjected to phosphorylation by GSK3 must first be phosphorylated by a different kinase at a serine/threonine approximately 4 residues c-terminal to the target serine/threonine, $\mathrm{S} / \mathrm{T}-\mathrm{X}-\mathrm{X}-\mathrm{X}-\mathrm{S} / \mathrm{T}(\mathrm{P})$ (Dajani et al., 2001; Ter Haar et al., 2001). According to the GSK3 crystal structure, the primed phosphorylation residue allows for binding to GSK3, as it occupies a binding pocket on GSK3 that contains positively charged amino acids, which allows subsequent phosphorylation of the targeted residue (Dajani et al., 2001; Ter Haar et al., 2001). As seen for the GSK3 $\beta$ substrate, von Hippel-Lindau (VHL), CKI initially phosphorylates serine 72 of VHL is required to allow subsequent recognition and phosphorylation by GSK3 $\beta$ on serine 68 (Hergovich et al., 2006). Interestingly, not all proteins follow this primed phosphorylation mechanism. Frame et al., in 2001 , mutated the phosphate binding pocket of GSK3$\beta$ and it did not affect activity towards nonprimed substrates like $\beta$-catenin (Polakis 2002; Frame et al., 2001). Furthermore, it is possible that Axin may bring both GSK3 and $\beta$-catenin in close proximity for subsequent $\beta$-catenin phosphorylation (Frame et al., 2001; Ikeda et al., 1998). In order for GSK3 to target specific 
substrates, 1) The substrate must be localized to specific compartments containing substrate 2) GSK3 $\beta$ requires signals to alter its localization to different compartments.

\subsubsection{GSK3 in disease: Diabetes, Alzheimer's disease, and Cancer}

GSK3 controls many important cellular processes that involve apoptosis, growth, and proliferation. Moreover, when GSK3 function is impaired or disrupted, it may alter cellular physiology, leading into a disease state. Many studies have highlighted GSK3 as a contributor to many diseases including diabetes, Alzheimer's disease (AD), and cancer (Beurel et al., 2015).

In normal physiology, the body is required to regulate and clear blood sugar (i.e. glucose) and use it to fuel cells with energy (Katsarou et al., 2017; DeFronzo et al., 2015). In order for glucose to be cleared, the body uses insulin, which is produced and released by the pancreas, which then stimulates specific cells (i.e. adipose, skeletal muscle) to robustly increase the rate of glucose uptake (Katsarou et al., 2017; DeFronzo et al., 2015). Importantly, in patients with diabetes, glucose metabolism is dysregulated due to difficulty in clearing blood glucose following a meal, due to either loss of beta-cells in the pancreas that normally produce insulin (type 1 diabetes) or poor response to insulin coupled to insufficient insulin production (type 2 diabetes) (Katsarou et al., 2017; DeFronzo et al., 2015). Notably, prior to establishment of type II diabetes (and continued during this disease), patients exhibit a state of insulin resistance, in which target tissues such as adipocytes and skeletal muscle cells have reduced or no response to insulin stimulation. 
GSK3 plays an important role in glycogen metabolism, by regulating glycogen synthase (Rylatt et al., 1980), and disruption of the regulation of glycogen synthase has been implicated in diabetes (Amar et al., 2011; Højlund et al., 2009; Højlund et al., 2003; Nikoulina et al., 2000; Lawrence and Roach, 1997). Indeed, dysregulation of GSK3 occurs in many cases of insulin resistance and diabetes (MacAulay and Woodgett, 2015). In many diabetes patient cases, GSK3 expression is upregulated in skeletal muscle (Eldar-Finkelman et al., 1999). In heart cells of diabetic rats, insulin stimulation was not able inhibit GSK3 via phosphorylation (Laviola et al., 2001), while in heart cells from control (non-diabetic) rats, activation of insulin receptors strongly inhibits GSK3 phosphorylation (Mancinelli et al., 2017; Beurel et al., 2015). In Chinese hamster ovary $(\mathrm{CHO})$ cells and hepatocytes that are treated with high glucose to induce a model of insulin resistance, GSK3 $\beta$ induces degradation of insulin receptor substrate (IRS), both of which one important component for insulin sensing/signalling (Leng et a., 2010) and found to be dysregulated in many diabetes cases (Lavin et al., 2016). Thus, these examples show the potential importance for GSK3 in contributing to diabetes and loss of mechanisms to regulate glucose homeostasis. As such, these contributions of GSK3 $\beta$ to systemic metabolism and of the disruption of GSK3 $\beta$ to metabolic pathophysiology indicate important role(s) for GSK3.

In $\mathrm{AD}, \mathrm{GSK} 3$ 's activity is dysregulated can promote the formation of plaques and neurofibrillary tangles (Zhang et al., 2018). A hallmark of AD is the formation plaques and neurofibrillary tangles in neurons, specifically generated from beta-amyloid and tau proteins, respectably, thus impairing neuronal function (Zhang et al., 2018; Araujo and Cotman, 1992; Kidd 1963). As seen in AD mouse and in vivo models, dysregulated GSK3 hyperphosphorylates tau protein, promoting tau aggregation, leading to neurofibrillary tangles and eventually 
neurodegeneration (Medina and Avila, 2013; Hernandez et al., 2001). In AD mouse models, GSK3 has been shown to regulate the gene transcription and expression of $\beta$-site APP-cleaving enzyme (BACE1) (Ly et al., 2013; Medina and Avila, 2013), which is an important protein for the cleavage of $\beta$-amyloid precursor protein (APP), that generates $\beta$-amyloid proteins found in $\beta$-amyloid plaques in AD (Zhang et al., 2018; Medina and Avila, 2013; De Strooper et al., 2010). In both examples, GSK3 regulates different aspects of cellular function implicated in $\mathrm{AD}$, from plaque formation and neurofibrillary tangles. While the disruption of GSK3 $\beta$ function leading to AD may reflect at least in part neuronal and brain-specific functions of GSK $3 \beta$ (and thus not described further here), these findings nonetheless inform about the broad range of cellular and systemic functions of GSK3 $\beta$.

Many lines of evidence also indicate that GSK3 also regulates aspects of cancer cell physiology. Interestingly, GSK3 may act either as a tumor promoter or a tumor suppressor, a critical distinction that appears to be largely due to cellular and tumor context (McCubrey et al., 2014). One example of a role of GSK3 $\beta$ in cancer is the regulation of $\beta$-catenin, which is an important transcription factor that regulates cellular processes including proliferation, survival, and migration by regulating the transcription of different target genes such as MYC (He et al., 1998), survivin (Kim et al., 2003), and matrix metalloproteinase 7 (Brabletz et al., 1999). GSK3 functions as a tumor suppressor by phosphorylating $\beta$-catenin, leading to proteosomal degradation (Mancinelli et al., 2017; Cohen and Frame, 2001). In contrast, Cao et al. in 2006 demonstrated that in ovarian cancer cells, elevated expression of GSK $3 \beta$ mediated increased expression of cyclin D1 (Cao et al., 2006), a protein involved in cell cycle progression, specifically by promoting the transition from G1 to S-phase (Sherr and Roberts, 2004). 
Furthermore, Ougolkov et al. in 2005 demonstrated that GSK3 $\beta$ controls the expression NFKB (Ougolkov et al., 2005), a transcription factor that controls the expression of anti-apoptosis genes (Sen and Baltimore, 2013). Indeed, in pancreatic cancers, active GSK3 $\beta$ increased the expression levels of NFkB, leading to cancer proliferation and survival (Ougolkov et al., 2005). With GSK3 capable of functioning as either a tumor suppressor or promoter in different cases of cancer, this suggests that there are aspects of GSK3 that remain to be uncovered. Here, I hypothesize that signals that control cellular localization, specifically nuclear localization of GSK3, could modulate GSK3 $\beta$ function to allow for context-specific functions of GSK3. Importantly, GSK3 phosphorylates and thus regulates many downstream effectors that are nuclear transcription factors, many of which exhibit constitutive nuclear localization. Thus, to understand how GSK3 activity regulates transcription and transcription factors, discussed are the main properties of transcription factors, regulation, and involvement cancer.

\subsubsection{Transcription factor regulation and activity}

Transcription factors (TF) modulate gene transcription and protein expression that lead to changes of cellular physiology including growth, proliferation, survival, and differentiation, including the important process of epithelial-mesenchymal transition (EMT) that is critical for invasion in many forms of cancer (Lambert et al., 2018; Bhagwat and Vakoc, 2015; Hao et al., 2013). Receptor signalling coordinates downstream signals to effectors that mediate the transcriptional activity of a particularly important TF, c-Myc, that control growth, proliferation, and survival. Furthermore, c-Myc controls many aspects of metabolism and may induce expansion of the mitochondria, increase glycolysis, and upregulate genes involved in biomass 
production (Dang 2015; Miller et al., 2012). In receptor signalling, RTK stimulation leads to Erk phosphorylation of serine 62 on c-Myc, which stabilizes c-Myc, which transiently accumulates to promote cell growth (Sears et al., 2000). Additionally, it was also found that Mad1 is an inhibitory heterodimer of c-Myc (Zhu et al., 2008). Thus, c-Myc requires RTK signalling to release the inhibitory function of Mad1 (Zhu et al., 2008). RTKs signals to downstream effectors S6K and p90 ribosomal kinase (RSK), that phosphorylate Mad1 on serine 141, promoting its proteosomal degradation (Zhu et al., 2008). Furthermore, c-Myc heterodimerizes with Max, promoting c-Myc activation, leading to the upregulation of c-Myc-dependent transcription of survival and growth genes (Zhu et al., 2008). In contrast to these mitogenic signals that lead to enhanced activity of c-Myc, active GSK3 3 phosphorylation on threonine 58 on c-Myc, promotes proteosomal degradation preventing transcriptional activity (Sears 2004). This demonstrates the existence of multistep processes that RTKs coordinate to regulate transcriptional activity of cMyc, some of which may critically depend on GSK3 $\beta$. How GSK3 $\beta$ is regulated to effect selective control of c-Myc under specific cellular contexts is poorly understood, which I examine in Chapter 3 and 4.

Another important TF, Zinc finger protein SNAL1 (Snail), is a protein that controls the process of EMT, which is the phenomena that epithelial cells undergo a coordinated change in differentiation status involving the loss of cell-to-cell contacts and upregulation of genes that allow the cell to become mesenchymal and thus more migratory (Kalluri and Weinberg, 2009). Once expressed, Snail down-regulates the expression of protein E-cadherin, which is important for making cell-to-cell contacts in epithelial monolayers (Wang et al., 2013). In the absence of growth factor signalling, GSK3 $\beta$ phosphorylates serine 96 (Xu et al., 2010), promoting Snail for 
proteosomal degradation and preventing EMT (Zhou et al., 2004). Inhibition of GSK3 $\beta$ via activation of PI3K and MAPK pathways lead to Snail activation, preventing transcription of Ecadherin inducing EMT (Zhou et al., 2004). These findings demonstrate the process of growth factor signalling to control the transcriptional activity of Snail for E-cadherin expression in EMT. GSK3 $\beta$ is regulated to effect selective control of Snail under specific cellular contexts is poorly understood, which I examine in Chapter 3 and 4.

Since TFs are master regulators of transcription, its activity must be precisely regulated. Therefore, dysregulation in TF activity may be problematic, as it may alter the cells functional outcome leading to a disease state like cancer.

\subsubsection{GSK3 $\beta$ substrate transcription factors in cancer}

TF dysregulation may reprogram the cells transcriptional activity, leading to an uncontrolled regulation of gene expression, and this is important in driving the growth and progression of some forms of cancer (Bhagwat and Vakoc, 2015; Lee and Young, 2013). Interestingly, a critical function of some transcription factors in cancer is to elicit changes in gene expression that lead to a particular set of cancer cells to resist chemotherapy treatments. Two TFs that will be discussed for their roles in contributing to cancer and chemoresistance are c-Myc and Snail, which are very relevant to this work given their reported regulation by GSK3 $\beta$.

MYC contributes to about $>50 \%$ of different cancers, where an upregulation of c-Myc is associated with poor prognosis and low rates of patient survival (Chen et al., 2018). c-Myc 
contributes to a broad range of cellular processes such as growth, proliferation, and cell division, so it is evident how dysregulation of c-Myc may alter these cellular processes to cancer phenotypes. In 2017, Lee et al. observed that MYC is amplified in cancer stem cells, and uncovered a novel mechanism by which upregulated c-Myc contributes to cell survival and chemotherapy resistance (Lee et al., 2017). Upregulation of c-Myc lead to enhanced expression of genes involved in mitochondrial biogenesis, as well as an increase in mitochondrial size and DNA content (Lee et al., 2017). Importantly, this increased mitochondrial biogenesis lead to an increased production of ROS, which in turn influenced the expression of hypoxia inducible factor $1 \alpha($ HIF-1 $\alpha)$ (Lee et al., 2017). HIF-1 $\alpha$ becomes an important mediator for resisting against chemotherapy treatment, that under treatment with chemotherapy drugs like paclitaxel, cancer cells were resistant to cell death (Lee et al., 2017). Furthermore, HIF-1 $\alpha$ promotes resistance to paclitaxel by activating the expression of interleukin 6 (IL-6), interleukin 8 (IL-8), and multidrug resistance 1 (MDR1), which key signals to activate survival signals (Samanta et al., 2014). c-Myc also contributes to cancer promoting metastasis (Wolfer and Ramaswamy, 2011; Chan et al., 2010; Wolfer et al., 2010), metabolic reprogramming to induce proliferation (Qing et al., 2010; Dang et al., 2007; Kim et al., 2007), and induce angiogenesis (Dews et al., 2010; Kine-Bamforth et al., 2004; Baudino et al., 2002).

Snail is an important TF that regulates genes in EMT, promoting mesenchymal and migratory phenotypes which is evident in many cancers. In 2009, Nawneet et al. demonstrated in ovarian cancer that dysregulation of Snail reprograms transcriptional activity to resists chemotherapy and promote survival (Nawneet et al., 2009). Treatment with the chemotherapy drug paclitaxel induced an increase in expression of Snail in ovarian cancer (Nawneet et al., 
2009). In turn, this increased expression of Snail repressed the expression of genes in the p53 pathway (Nawneet et al., 2009), a pathway involved in regulating apoptosis and cell cycle arrest upon DNA damage and other forms of cellular stress (Joerger and Fersht, 2016). Thus, Snailmediated repression of genes in the p53 pathway prevents the activation of apoptosis pathways (Nawneet et al., 2009). By this model, chemotherapy resistance occurs by chemotherapy treatment to influence the upregulation of genes that repress p53 stress pathways, inhibiting apoptosis mechanisms and enhanced survival. Snail also contributes to cancer pathways by coordinating metastasis mechanisms (Olmeda et al., 2007), resistance to programmed cell death (Kajita et al., 2004), and promoting tumor growth (Taki et al., 2018; Kudo-Saito et al., 2009).

The control of TF function by cytosolic signals (such as RTKs) requires communication of these signals from the cytosol to the nucleus. GSK3 may regulate a number of TFs but also receives signals from the cytosol. Thus, it is important to understand and consider the nuclear pore complex (NPC) and transport of molecules in and out of nucleus.

\subsubsection{Nucleus: transcriptional and signalling regulation}

The nucleus is the brain and control centre of the cell, containing important information to regulate various processes such as transcription and signalling pathways. The nucleus is enclosed by a double lipid membrane (inner and outer membrane) called the nuclear envelope (NE), which acts as a physical barrier (Cautain et al., 2015). The NE is not entirely closed off from the cytosol, as it contains many nuclear pore complexes (NPC) throughout the NE, which acts as gatekeepers to allow the selective permeability of different biomolecules in and out of the 
nucleus (Grossman et al., 2012). Examples of molecules that cross the NPC include import of newly synthesized TFs, DNA/RNA polymerase, and histones, and export of ribosomes and RNA (Kabachinski and Schwartz, 2015), There are specific mechanisms for the translocation of proteins and mRNA in and out of the nucleus, which will be discussed in the next section.

\subsubsection{Import and Export of biomolecules: selectivity}

To import and export different biomolecules to and from the nucleus through the NPC, three specific criteria is required: 1) Nuclear import or export sequences 2) Nuclear receptors for specific cargo and 3) protein machinery that provides directionality. Proteins that are capable of undergoing import into the nucleus require an NLS (McLane and Corbett, 2009) and in contrast, proteins that are primed for export out of the nucleus use a nuclear export sequence (NES) (Cautain et al., 2015; Wente and Rout, 2010). NLS are typically comprised of a motif that contains $\sim 5-8$ basic amino acids (although vary), while NES contain hydrophobic residues (Cautain et al., 2015; McLane and Corbett, 2009; Dingwall and Laskey, 1991). Both NLS and NES are recognized by a family of nuclear receptor proteins called Karyopherins, which contain subfamilies of proteins called importins that recognize NLS and exportins that recognize NES containing cargo (Fig. 1.18) (Cautain et al., 2015; McLane and Corbett, 2009; Dingwall and Laskey, 1991). Examples of importins and the cargo recognized include importin- $\alpha 5$ and signal transducer and activator of transcription 3 (Stat3) (Ma and Cao, 2006), importin-9 and c-Jun (Waldmann et al., 2007), importin- $\alpha 3$ and NFאB (Fagerlund et al., 2005). Examples of exportins and the cargo recognized include exportin-1 and cyclin D1 (Benzeno and Diehl, 2004) and exportin-6 and profiling-actin complexes (Stüven et al., 2003). Currently, GSK3 $\beta$ does not have any known interacting importin binders but frequently rearranged in T-cell lymphomas/GSK-3- 
binding protein (Frat/GBP) has been identified as an exportin for GSK3 (Franca-Koh et al., 2002).

The final important component for nuclear translocation is the requirement for directionality. The protein responsible for mediating cargo directionality to be either nuclear or cytosolic is a protein GTPase called Ran (Kim et al., 2017; Wente and Rout, 2010; Kau et al., 2004). In the nucleus, Ran exists in the GDP bound state. The nuclear enrichment of Ran-GTP is ensured by the action of nuclear-enriched Ran GEF, which exchanges GDP for GTP on Ran, causing nuclear accumulation of Ran-GTP (Cautain et al., 2015). For nuclear export, Ran-GTP binds to protein complex containing cargo with a NES and exportins (Fig. 1.18) (Kim et al., 2017; Wente and Rout, 2010; Kau et al., 2004). The Ran GTP/cargo/exportin complex is recognized by the NPC to signal for export out of the nucleus. Upon reaching the cytosol, RanGTP is subject to hydrolysis of GTP by a cytosol-enriched Ran GAP, causing dissociation of the cargo (Fig. 1.18) (Kim et al., 2017; Wente and Rout, 2010; Kau et al., 2004). For nuclear import, protein cargo containing NLS bind to importins (in the absence of Ran-GTP, hence in the cytosol), which are recognized by the NPC, leading to nuclear import and dissociation of cargo (Fig. 1.18) (Kim et al., 2017; Wente and Rout, 2010; Kau et al., 2004). Ran GDP found within the cytosol are also reimported through other importins, enter the nucleus, exchange GDP to GTP by Ran GEFs, allowing for the next round of export (Kim et al., 2017; Wente and Rout, 2010; Kau et al., 2004).

GSK3 does contain properties for nuclear import mechanisms as it contains a noncanonical NLS, which may potentially be important for its translocation into the nucleus. It is not 
well understood if GSK3 follows the canonical translocation mechanisms via Ran dependent import to regulate downstream effectors such as c-Myc and Snail, and how signals that may regulate GSK3 $\beta$ nuclear localization can impact c-Myc and Snail levels and function.

\subsubsection{Rationale and Hypothesis}

It is important to understand the intertwined regulation of cell physiology by mitogenic and metabolic signalling and how these signals converge to regulate the activity and function of GSK3 to control the physiology of both healthy cells and cancer cells. The mechanism of how mitogenic and metabolic signalling converge on mTORC1 activity to regulate GSK3 function and localization has yet to be elucidated. Thus, doctoral research aimed to understand how mitogenic signalling (EGFR-PI3K-Akt) and metabolic signalling (energy and amino acid availability) affects GSK3 function and regulation of its compartmentalization within the cell. I hypothesize that regulation of GSK3 spatiotemporal dynamics within cells is critical to the regulation of cell physiology. To understand how mitogenic and metabolic signals regulate GSK3, I look to understand three aims/objectives:

1) Determine how mitogenic receptor signalling and plasma membrane clathrin nanodomains control Akt activation

2) Determine how mitogenic and metabolic signalling control GSK3 $\beta$ activity and localization

3) Resolve how the various metabolic signals that control mTORC1 activity regulate GSK3 $\beta$ 
1.10 Figures 

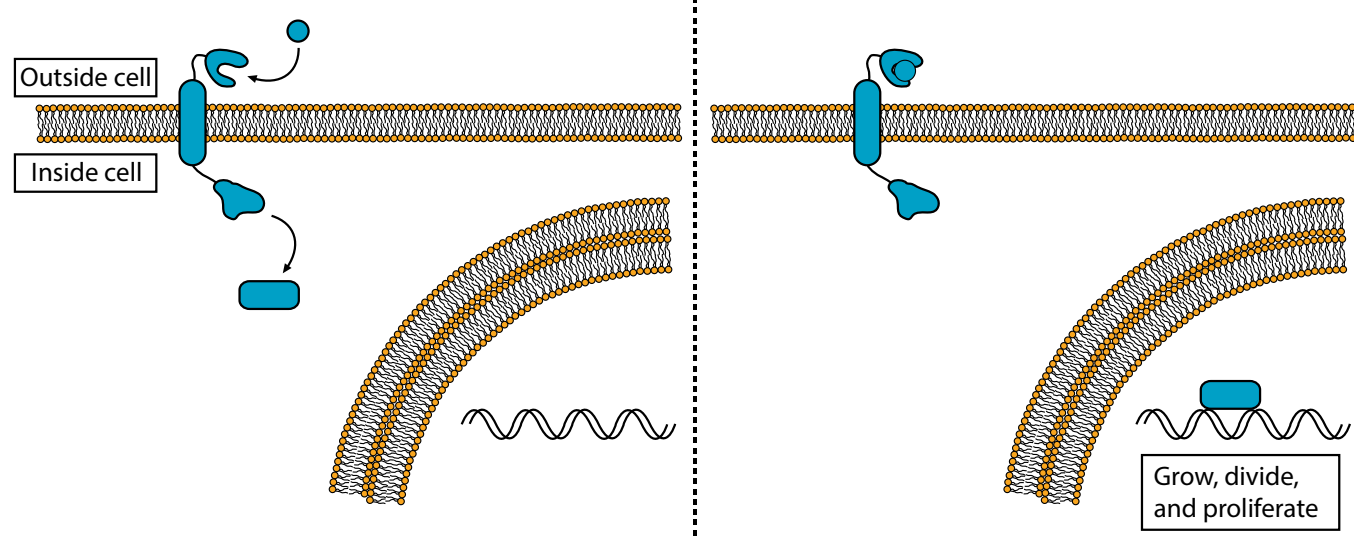

Figure 1. 1 Cells sense environmental cues to alternate its cell physiology. Receptors are the communicators between the environment and its internal signalling machinery. Receptors stimulated leads to changes in transcriptional activity alternating cellular physiology such as proliferation, cell division, and proliferation. 


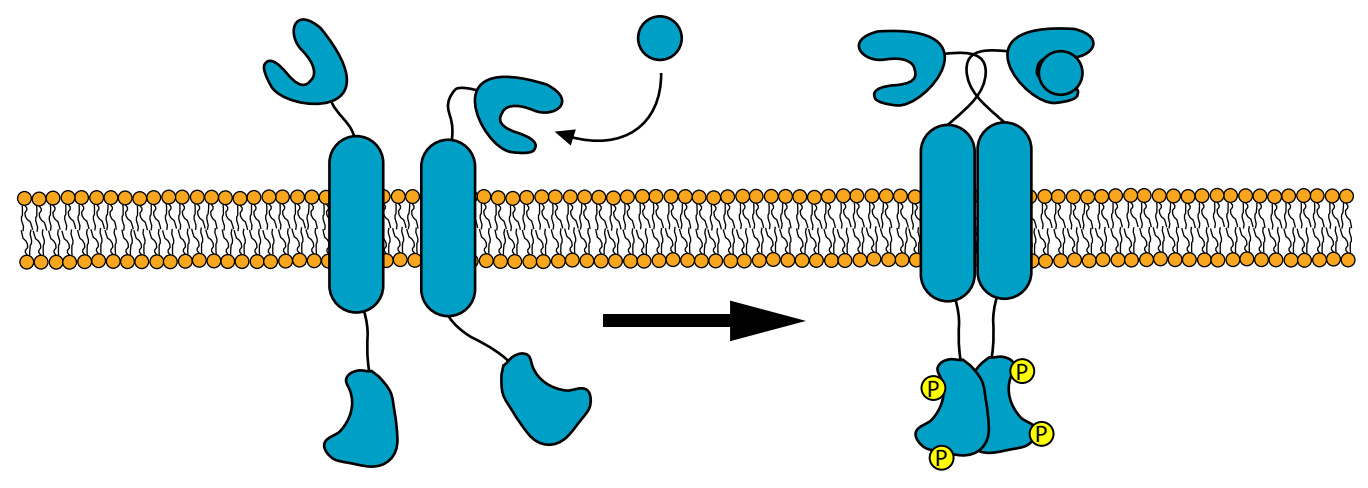

Figure 1.2 Ligand binding leads to receptor activation. EGFR binds to its ligand EGF, leading to dimerization, and autotransphosphorylation in the c-terminal domain of the receptor. Phosphorylation sites serve as docking sites for proteins, activating other signalling pathways. 


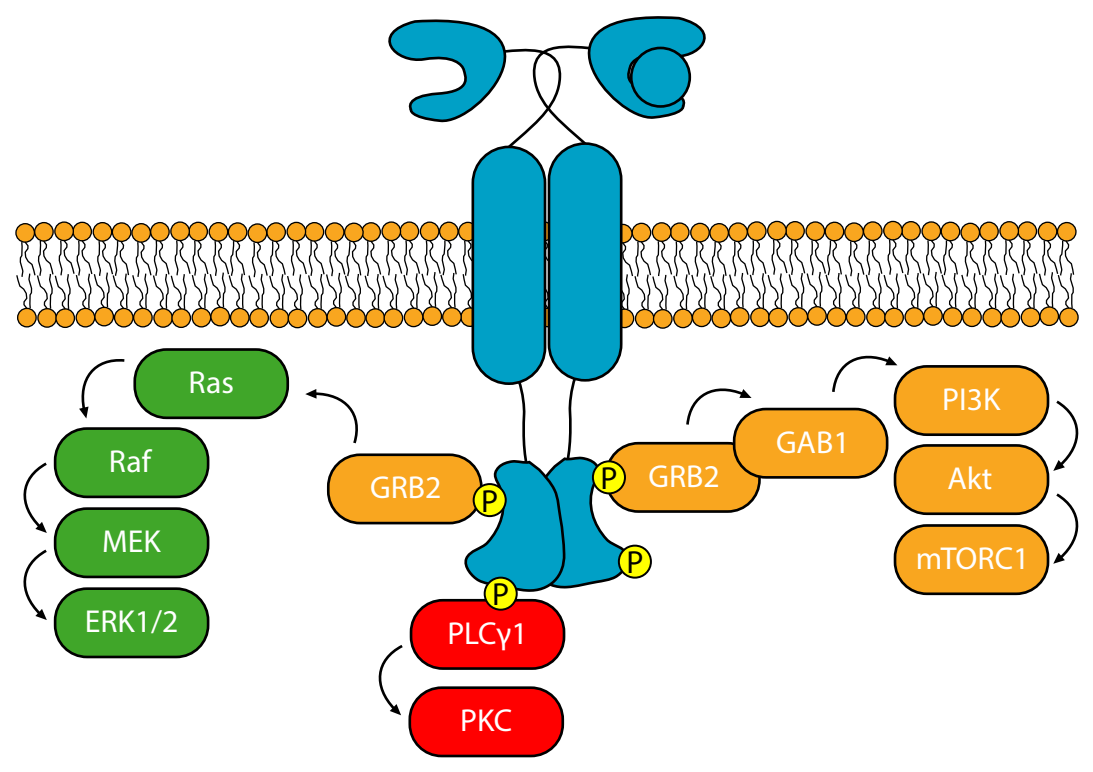

Figure 1.3 EGFR activates multiple signalling pathways Phosphorylation residues acts as docking sites for different signalling pathways including Ras/Raf/MEK/Erk, Gab1/PI3K/Akt/mTORC1, PLC $\gamma 1 /$ PKC, and others. 


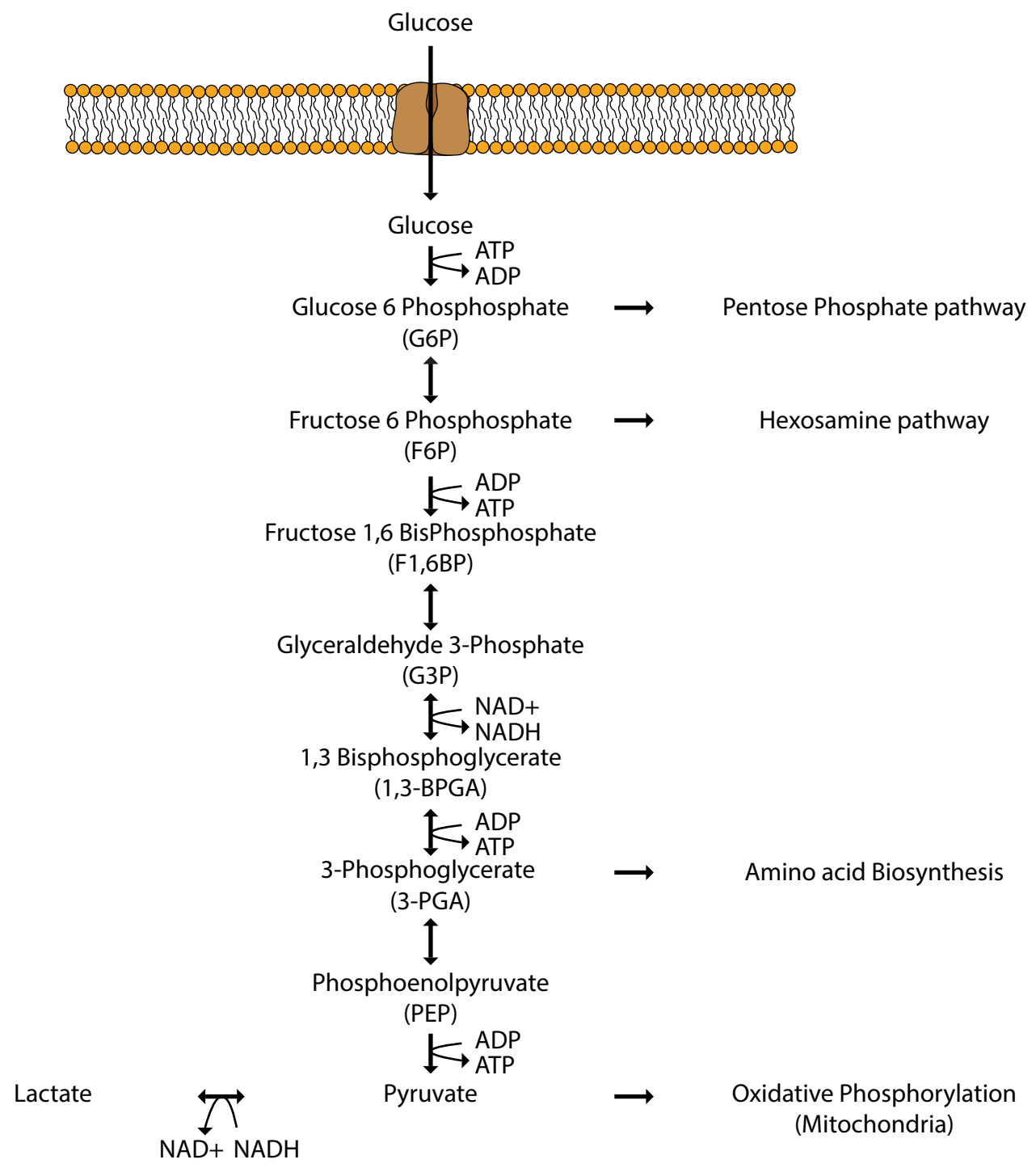

Figure 1. 4 Glycolysis and intermediates used in separate pathways. Shown is the process of glycolysis and the intermediates formed to generate energy (ATP) and cofactors (NAD+). Intermediates in the glycolysis pathway may be shuttled to other metabolic pathways for the formation of nucleotides (Pentose Phosphate pathway), glycans (Hexosamine pathway), and amino acids. 


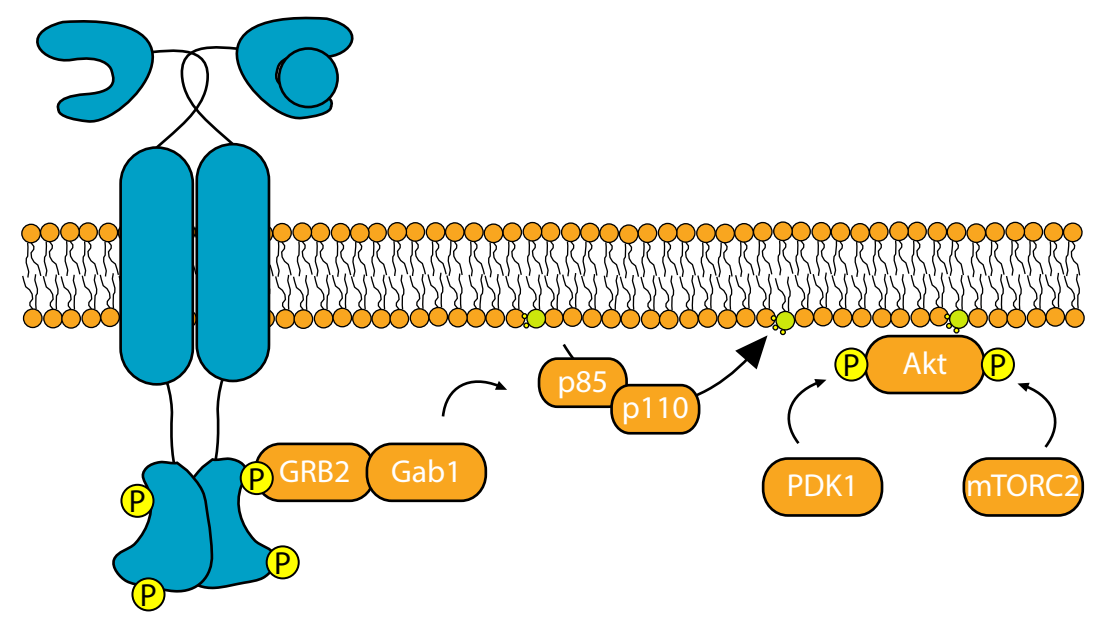

Figure 1. 5 EGFR regulates the PI3K and Akt signalling pathway. Activated EGFR recruits scaffold protein Grb2 and Gab1. Furthermore, Gab1 recruits PI3K, converting PI(4,5)P2 to $\mathrm{PI}(3,4,5) \mathrm{P} 3$. Subsequently, $\mathrm{PI}(3,4,5) \mathrm{P} 3$ recruits Akt leading to full activation and phosphorylation via PDK1 and mTORC2. 

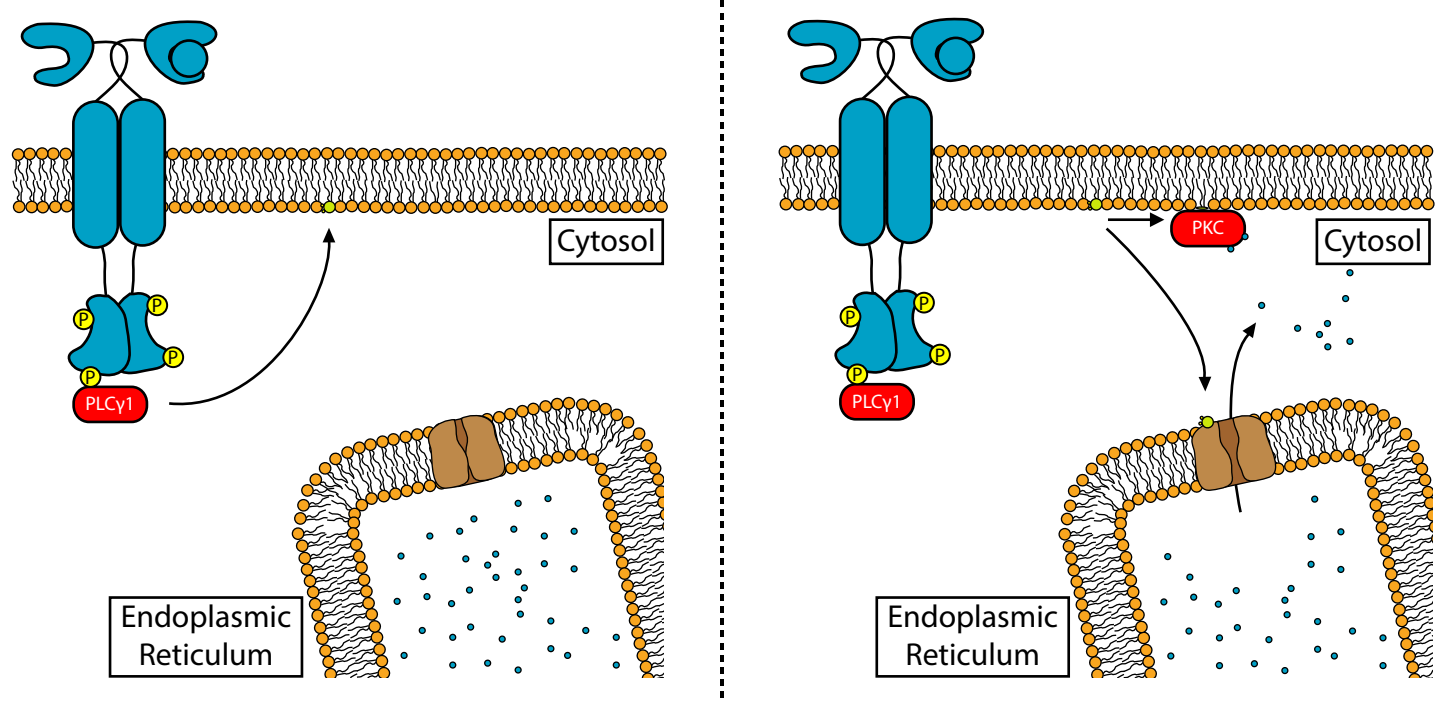

Figure 1.6 EGFR regulates the PLC $\gamma 1$ signalling pathway. Activated EGFR recruits and activates PLC $\gamma 1$ which breaks down PI(4,5)P2 into DAG and IP3. Furthermore, DAG and IP3 act as secondary messengers that bind to PKC and IP3 calcium channels respectably. Calcium released in the cytosol bind to many substrates acting as a tertiary messenger. 


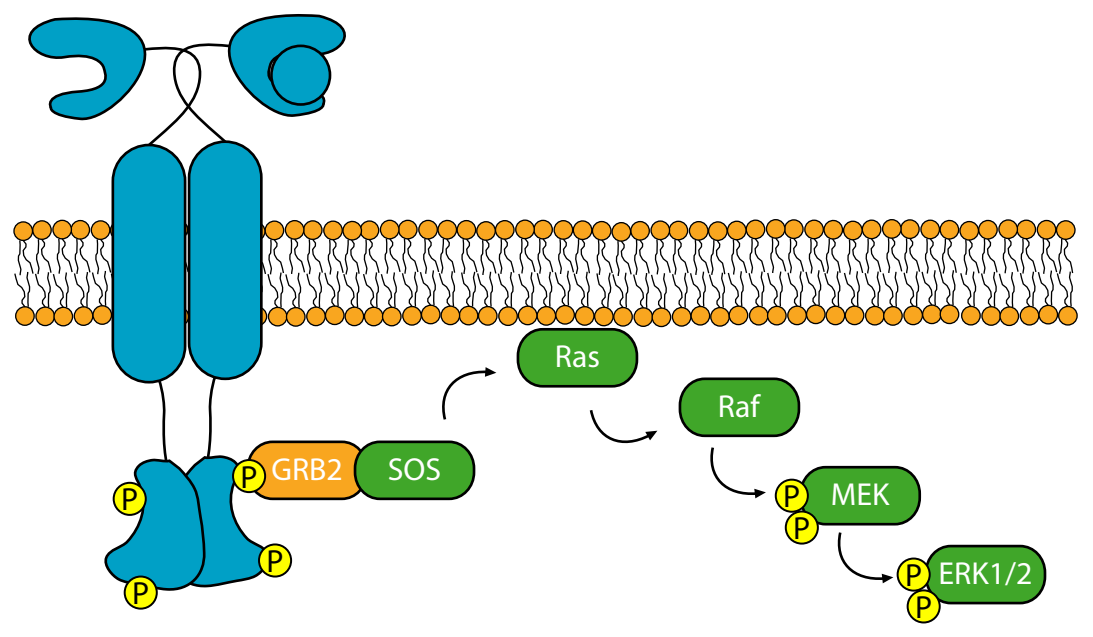

Figure 1. 7 EGFR regulates the MAPK signalling pathway. Activated EGFR recruits and activates the MAPK signalling by initially recruiting scaffold protein Grb2 and SOS.

Furthermore, SOS GEF activity, activates Ras and downstream targets, Raf, MEK, and ERK 1/2. 

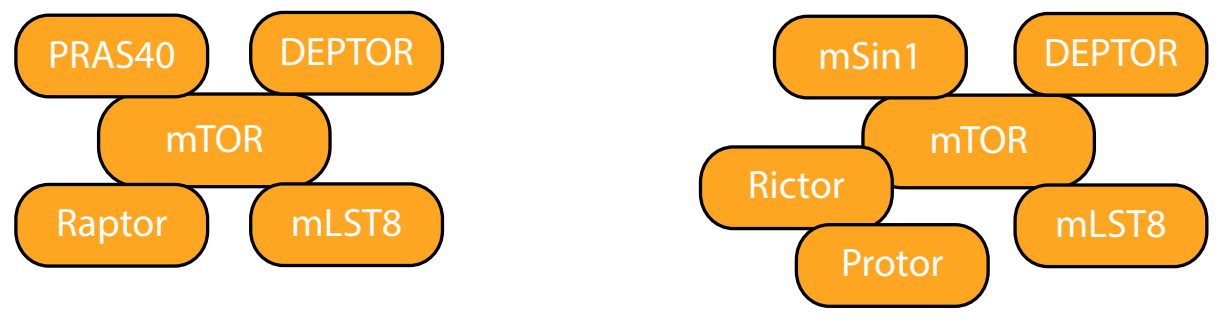

Figure 1. 8 mTORC1 and mTORC2: complex differences. mTOR has two different complexes, depending on the protein subunits recruited. mTORC1 contains subunits PRAS40, DEPTOR, Raptor, and mLST8, while, mTORC2 contains subunits mSIN1, DEPTOR, Rictor, Protor, and mLST8. 


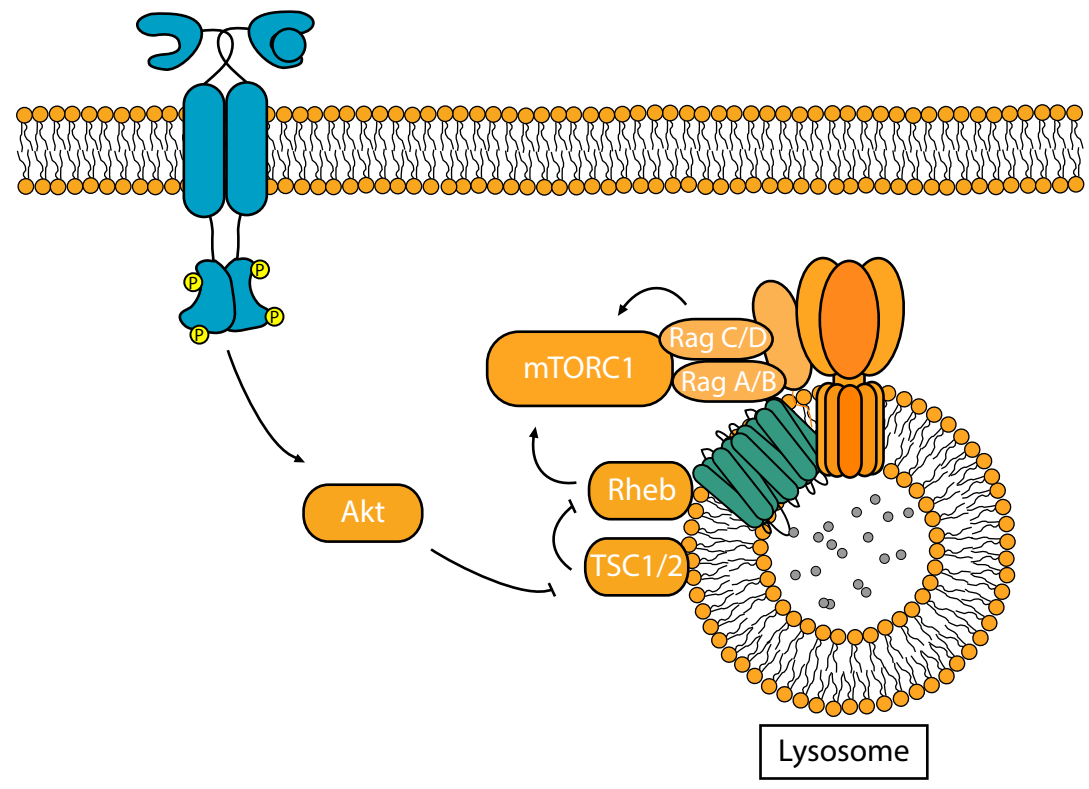

Figure $1.9 \mathrm{mTORC1}$ is regulated by both mitogenic and metabolic signalling. Two separate pathways are required to activate mTORC1. Lysosomal pools of amino acids engage and recruit mTORC1 to the lysosomal, while activated Akt inhibits TSC 1/2, allowing for Rheb dependent activation of mTORC1. 


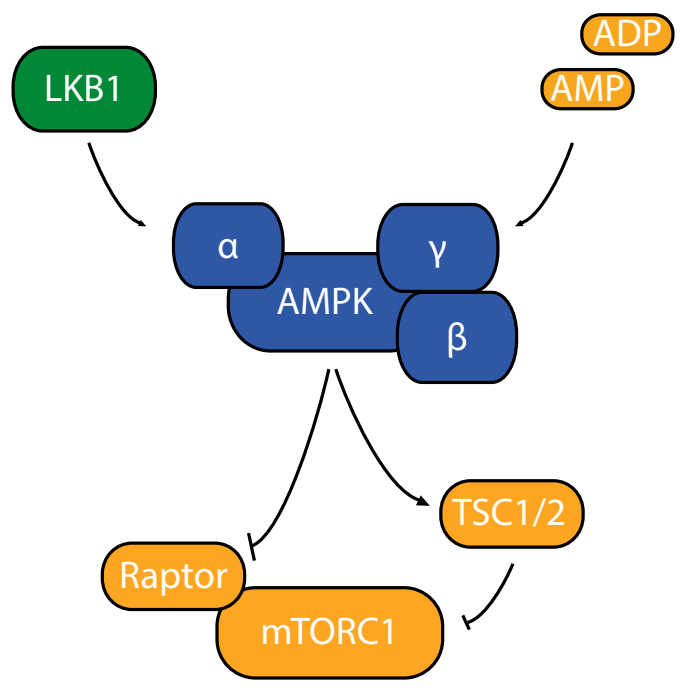

Figure 1. 10 AMPK regulates mTORC1 by sensing energy changes. In energy depletion conditions, AMPK is activated promoting the negative regulation of mTORC1 by activating TSC1/2 and inhibiting mTORC1 subunit Raptor. Also a regulator of AMPK activity is LKB1, which is also regulated by energy stress. 


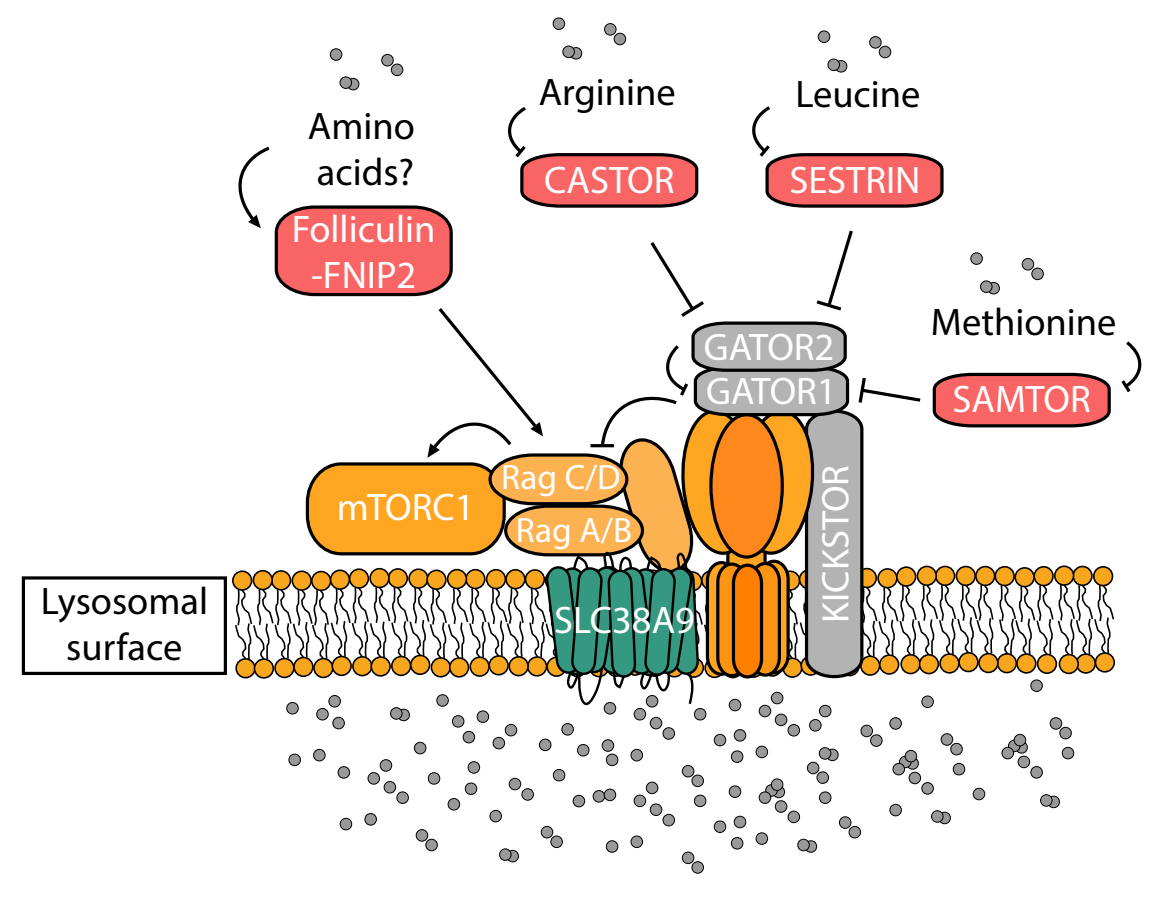

Figure 1. 11 mTORC1 amino acid sensing abilities regulated by multiple factors. mTORC1 is regulated by both intraluminal and cytosolic pools of amino acids. SLC38A9 senses intraluminal pools of amino acids to signal to both the V-ATPase and Ragulator complex, activating and recruiting mTORC1 to the surface of the lysosome. Furthermore, GATOR 1/2 complex indirectly senses cytosolic pools of amino acids through SAMTOR (methionine), SESTRIN (leucine), and Castor (Arginine), subsequently regulating activating rag proteins for mTORC1 upregulation. Folliculin-FNIP2 senses amino acids and directly upregulates rag proteins for $\mathrm{mTORC} 1$ activation. 


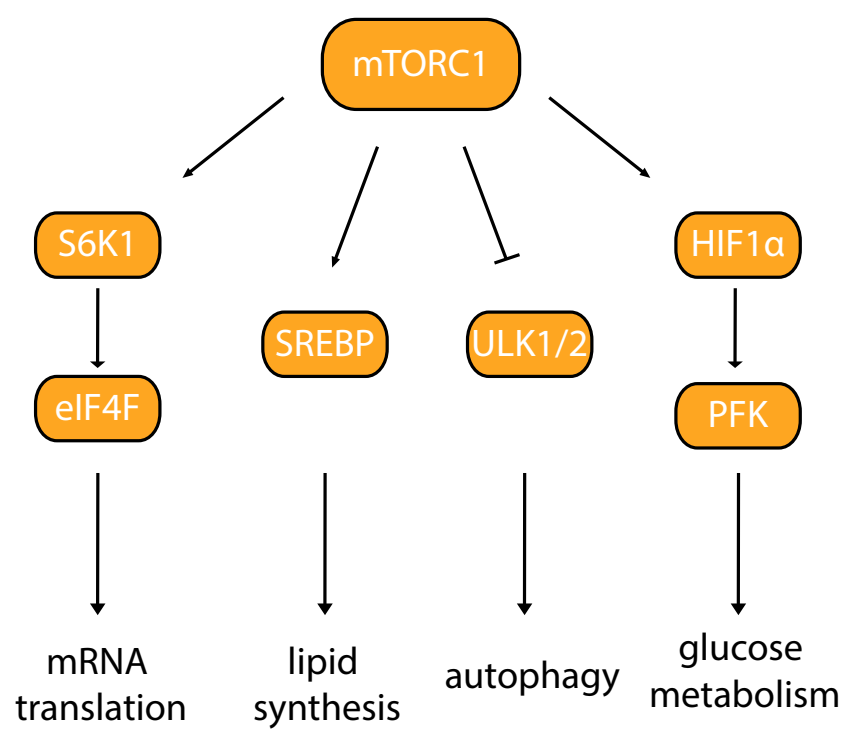

Figure 1. 12 mTORC1 activation leads to changes in translation and metabolism. mTORC1 regulates many downstream targets that lead to changes in mRNA translation (S6K1/eIF4F complex), lipid synthesis (SREBP), inhibition of autophagy (ULK1/2), and glucose metabolism (HIF1 $\alpha /$ PFK). 


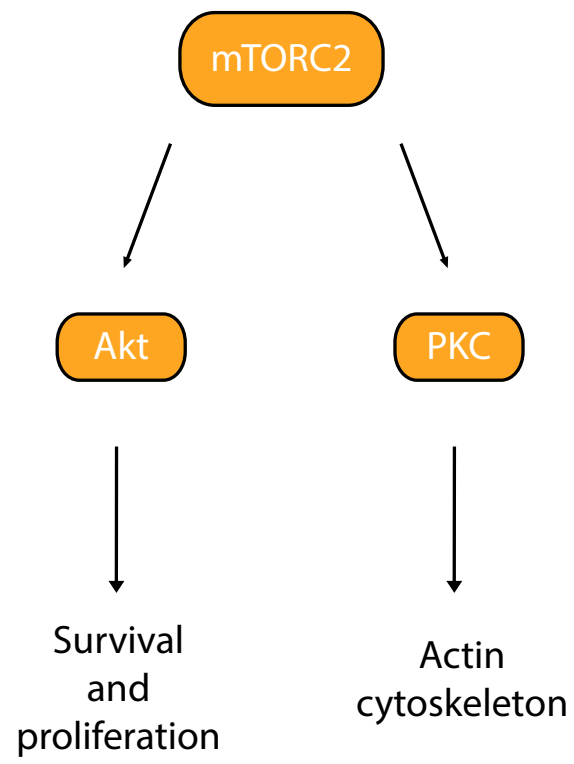

Figure 1. 13 mTORC2 activation leads to changes in cell survival and cytoskeleton

remodeling. $\mathrm{mTORC} 2$ regulates an important kinase, Akt, which regulates a variety of different substrates that control processes in cell survival and proliferation. Furthermore, mTORC2 also regulates actin cytoskeleton dynamics through $\mathrm{PKC}$ 


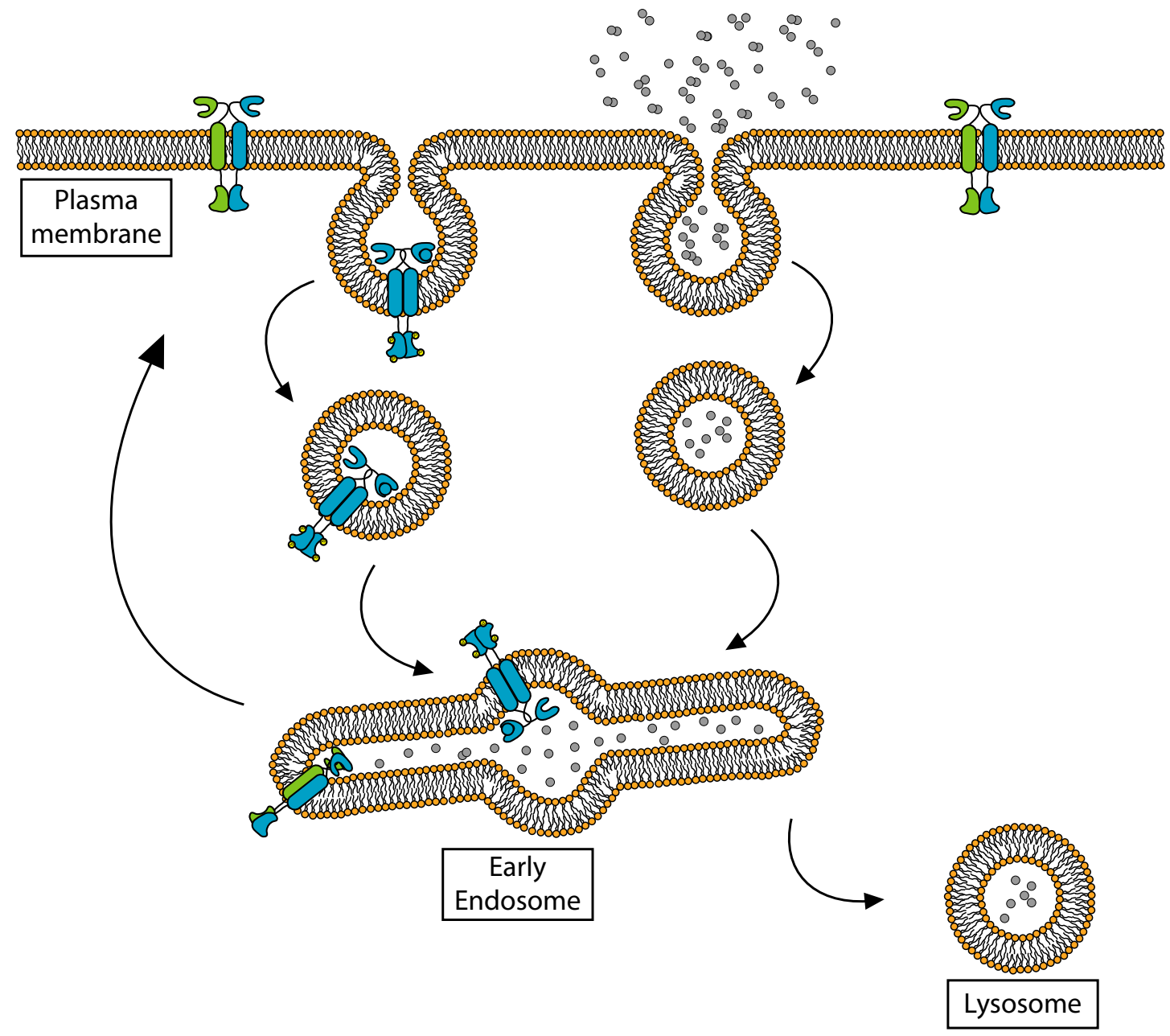

Figure 1. 14 Membrane traffic involves dynamic movement of material from different membrane compartments. Shown are the multiple events found within the cell during membrane traffic. 1) Cargo (receptors and nutrients) are internalized via endocytosis 2) Vesicle containing cargo is trafficked early endosome 3) Cargo from the early endosome are recycled back to the plasma membrane 4) Cargo from the early endosome may also be trafficked to the lysosome for subsequent degradation. 


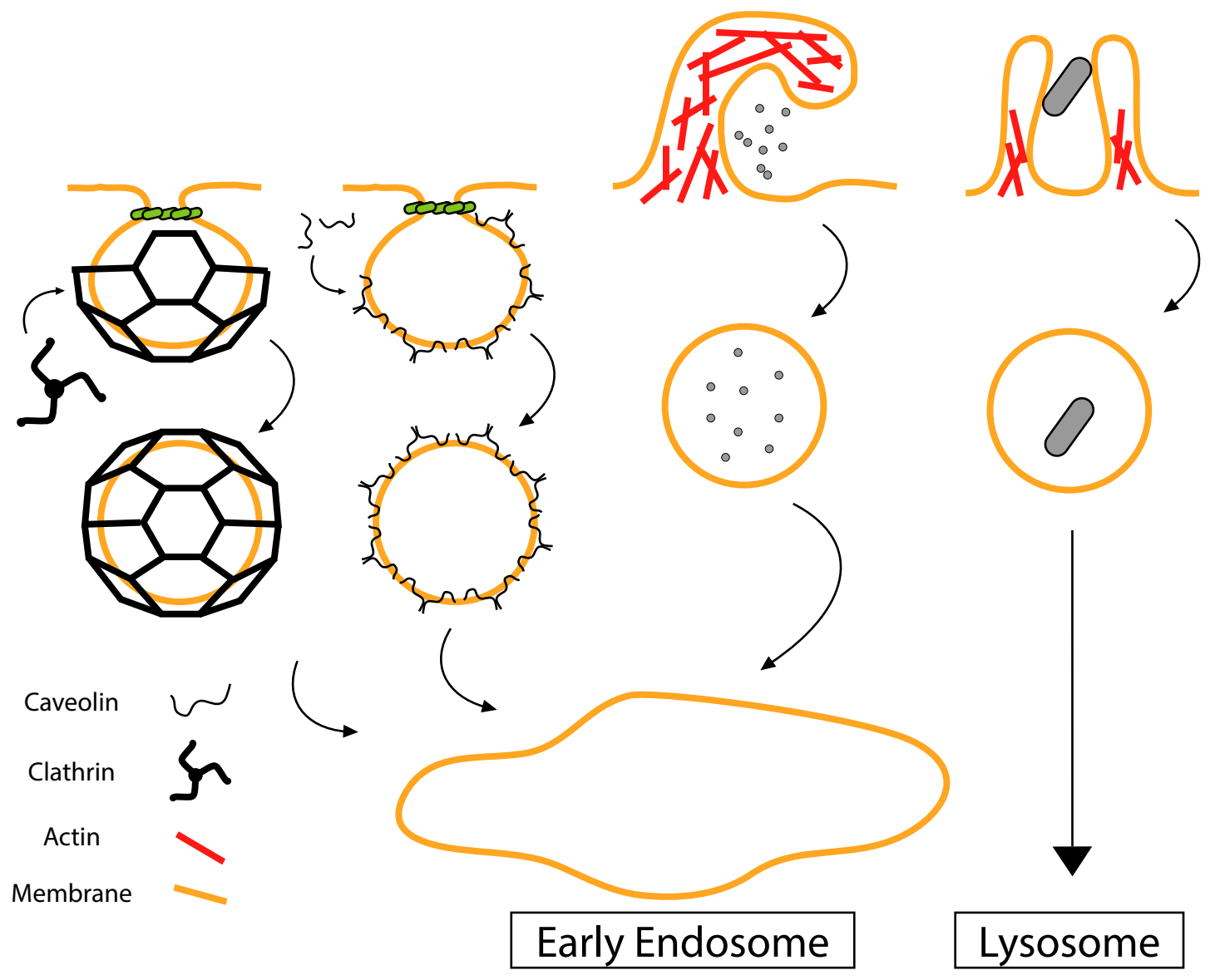

Figure 1. 15 Multiple endocytosis classes involve different mechanisms of internalization for specific cargo. Shown are 4 mechanisms of internalization, each of which use different proteins and some internalize different cargo. 1) Clathrin mediated endocytosis uses clathrin to internalize material such as protein receptors 2) Calveolin endocytosis uses Calveolin to internalize cargo material from the plasma membrane 3) Macropinocytosis is actin mediated internalization, which uses membrane ruffles to internalize non specific material 4) Phagocytosis is also an actin mediated internalization, which internalizes material such as pathogens and trafficked straight to the lysosomal for degradation. 


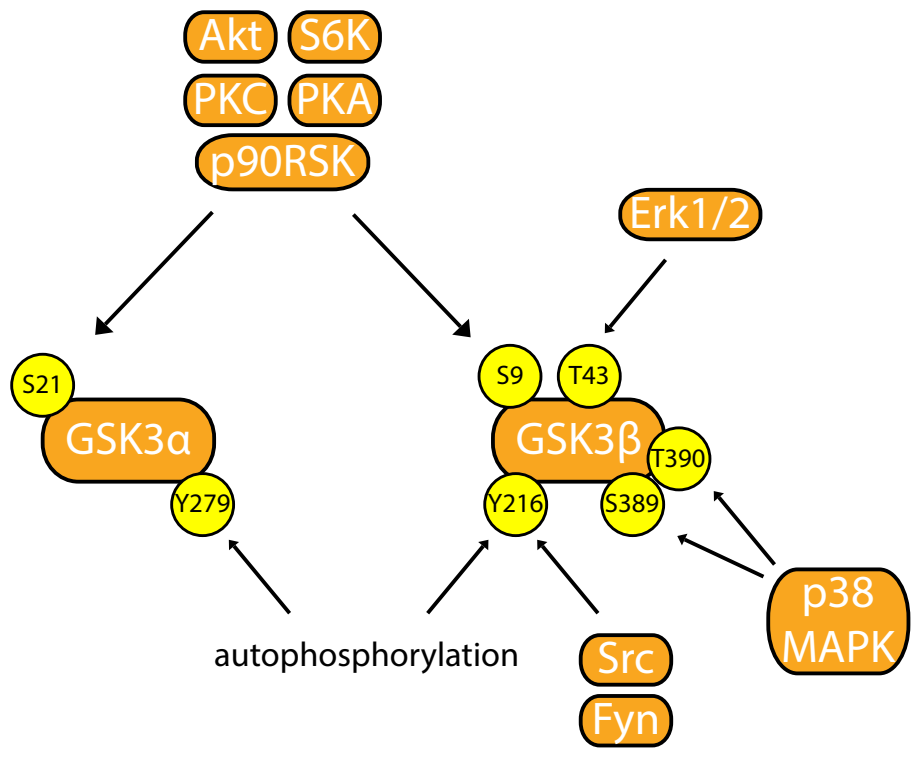

Figure 1. 16 GSK3 phosphorylation via different substrates. Shown are different substrates that regulate GSK3 activity, each of may enhance or inhibit GSK3 kinase activity. S21 on GSK $3 \alpha$ and S9 on GSK3 $\beta$ inhibit their kinase activity, in contrast, Y279 GSK $3 \alpha$ and Y216 on GSK3 $\beta$ enhance kinase activity. Other residues have been identified on GSK3 $\beta$, T43, S389, and T390 phosphorylation leads to the increased affinity for phosphorylation of S9 to inhibit GSK3 $\beta$ kinase activity. 


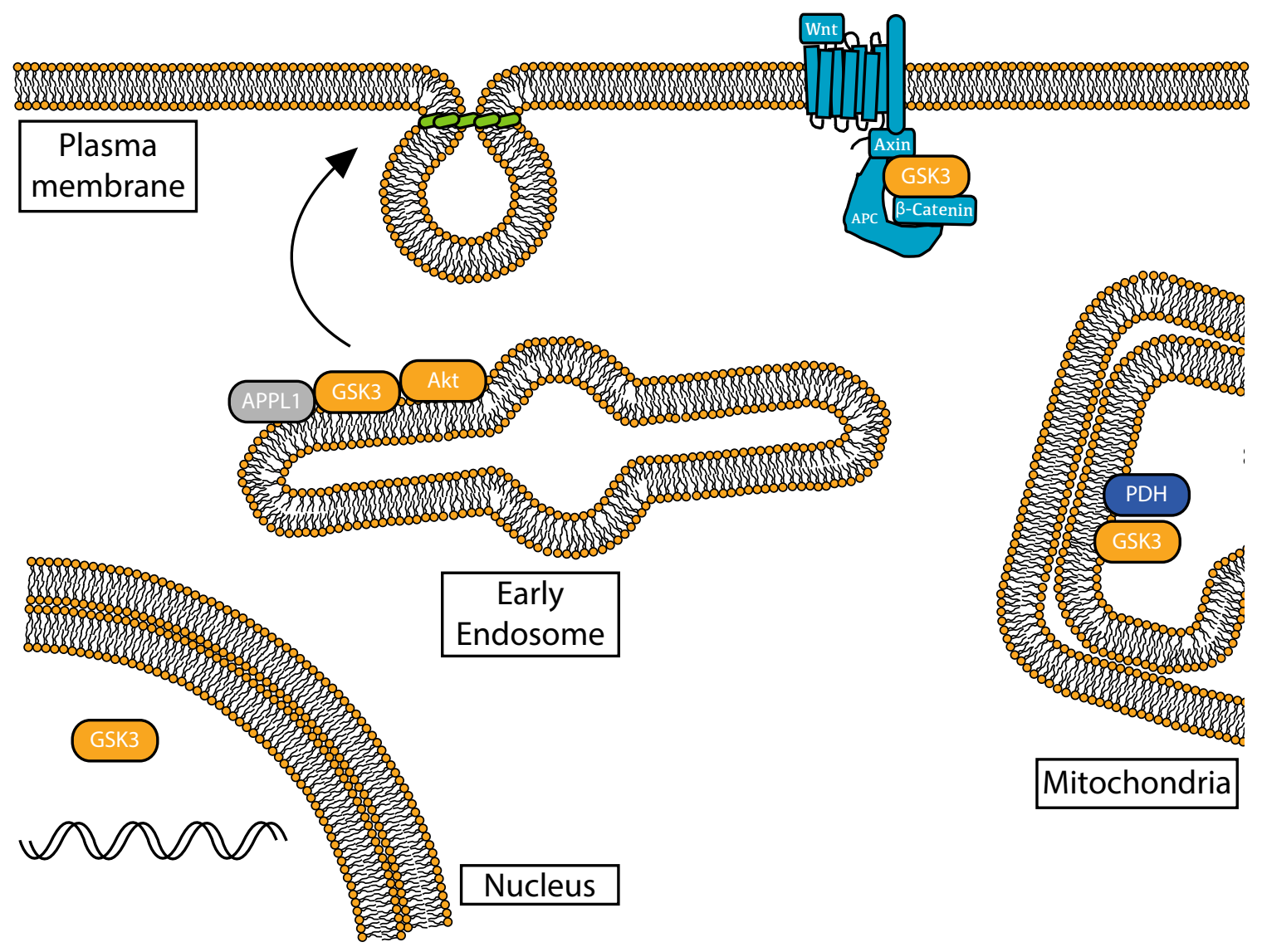

Figure 1.17 GSK3 is localized to many membrane compartments. Shown are the multiple compartments GSK3 may be localized to. GSK3 may be localized at the plasma membrane, early endosome, mitochondria, and within the nucleus. Each compartment allows for different interactions between multiple substrates. 

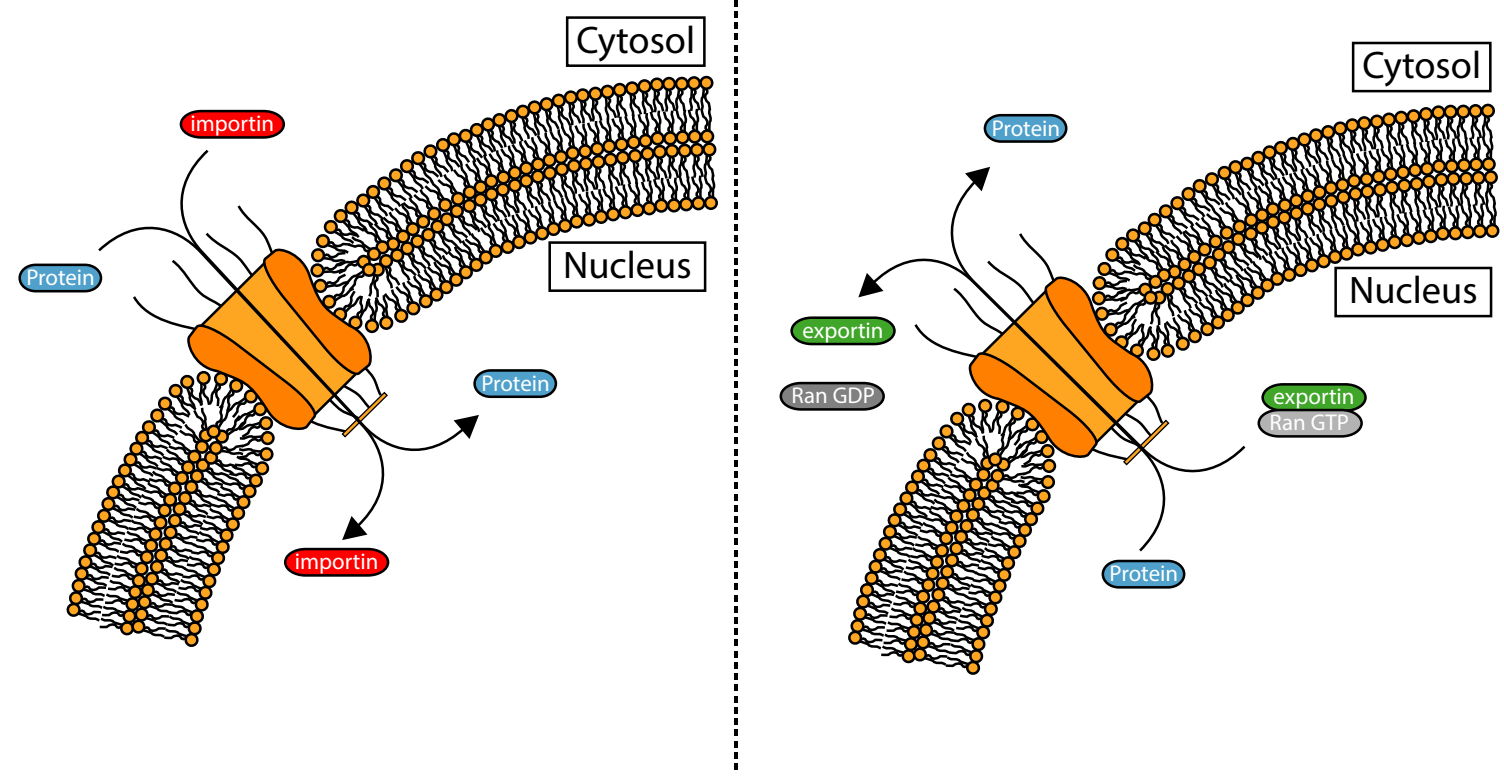

Figure 1.18 Dynamic nuclear import and export mediated by Ran GTPase. Proteins are shuttled between the nucleus depending on different properties. 1) Nuclear import or export sequence (NLS or NES) 2) Nuclear import or export receptors (importins or exportins) 3) Ran GTPase for directionality, where Ran GTP promotes export of specific proteins. 
Chapter 2: Materials and Methods 


\section{$\underline{2.1 \text { Materials }}$}

Antibodies targeting specific proteins were obtained as follows: EGFR (Y1068; \#44788G) (Invitrogen, ThermoFisher (Rockford, IL)), Gab1 (Y627; cat \# 3233), pan Akt (cat \# 2920), Akt (S473; cat \# 4051), total PLC 1 (cat \# 2822), total ERK (cat \# 9102), ERK (T202/Y204; cat \# 9101), GSK3 $\beta$ (cat \# 9832), GSK3 $\alpha$ (cat \# 4815), GSK3 $\alpha / \beta$ (cat \#5676) phospho-GSK3ß (S9; cat \# 9323) actin (cat \# 8456), HA -epitope (cat \# 3724 or 2367), EEA1 (cat \# 3288), LAMP1 (cat \# 15665), APPL1 (cat \# 3858), ULK1 (cat \#8054) (Cell Signaling, Danvers, MA), dynamin-1 (ab52852) (Abcam, Cambridge, MA), and clathrin (sc -12734) and Lamin A/C (sc -6215) (Santa Cruz Biotechnology, Dallas, TX). Horseradish peroxidase or fluorescently -conjugated secondary antibodies were purchased from Cell Signaling Technology (Danvers, MA). DAPI Nuclear staining was purchased from ThermoFisher (Rockford, IL).

Ran cDNA constructs tagged to HA, including WT, T24N and G19V forms in pKH3 were generously provided by Dr. Ian Macara (Vanderbilt University School of Medicine, Nashville, TN) (Carey et al., 1996)). GSK3 $\beta$ cDNA constructs, including HA -tagged WT and S9A forms in pcDNA3 were generously provided by Dr. Jim Woodgett (Lunenfeld -Tanenbaum Research Institute/Mount Sinai Hospital, Toronto, ON) (Stambolic and Woodgett, 1994). Rab7 constructs, including WT and T22N, were generously provided by Dr. Richard Pagano (Mayo Clinic and Foundation, Rochester, MN) (Choudhury et al., 2002). 


\section{$\underline{2.2 \text { Cell lines, cell culture and inhibitor treatment }}$}

Wild -type human retinal pigment epithelial cells (ARPE -19; RPE herein) were obtained from American Type Culture Collection (ATCC, Manassas, VA) and cultured as previously described (Delos Santos et al., 2017) with DMEM/F12 (Gibco, ThermoFisher Scientific, Waltham, MA) containing $10 \%$ fetal bovine serum, $100 \mathrm{U} / \mathrm{ml}$ penicillin and $100 \mu \mathrm{g} / \mathrm{ml}$ streptomycin. Cells were then incubated at $37 \mathrm{C}$ and $5 \% \mathrm{CO}_{2}$. MDA-MB-231 cells were obtained from ATCC and cultured as previously described (Fekri et al., 2016) with RPMI media 1640 (Gibco) containing 10\% fetal bovine serum, $100 \mathrm{U} / \mathrm{ml}$ penicillin and $100 \mu \mathrm{g} / \mathrm{ml}$ streptomycin and incubated at $37 \mathrm{C}$ and $5 \% \mathrm{CO} 2$.

All inhibitor treatments were performed (alone or in combination) for $1 \mathrm{~h}$ prior to experimental assays unless otherwise indicated, as follows: $10 \mu \mathrm{M}$ CHIR99021 (Abcam, Cambridge, MA), $1 \mu \mathrm{M}$ rapamycin (BioShop, Burlington, ON), $10 \mu \mathrm{M}$ LY294002 (Cell Signaling Technologies), $5 \mu \mathrm{M}$ Akti -1/2 (Sigma - Aldrich, Oakville, Canada), $1 \mu \mathrm{M}$ concanamycin A (BioShop). Following inhibitors were treated for 20 minutes: pitstop2 (10 $\mu \mathrm{M})$ or $80 \mu \mathrm{M}$ dynasore (all inhibitors obtained from Abcam, Cambridge, MA) or a corresponding volume of dimethyl sulfoxide (DMSO; vehicle control). Amino acid starvation and refeeding treatments were performed by incubation in Earle's Balanced Salt Solution (EBSS, Gibco) for 1 prior to experimental assay and refeed with amino acids leucine $0.4 \mathrm{mM}$ (cat \#61-90-5) or arginine 0.4 mM (cat \#74-79-3) (both amino acids purchased from BioShop, Burlington, ON) for 15 minutes. 


\section{$\underline{2.3 \text { Plasmid and siRNA transfections }}$}

To perform DNA plasmid transfections, Lipofectamine 2000 (Thermo Fisher Scientific) was used according to the manufacturer's instructions and as described previously (Bone et al., 2016). Briefly, cells were incubated for $4 \mathrm{~h}$ with Lipofectamine 2000 reagent and appropriate plasmid in Opti-MEM (Gibco) at a 3:1 ratio. Subsequently, this transfection solution was removed, and cells were incubated in fresh cell growth medium at $37^{\circ} \mathrm{C}$ and $5 \% \mathrm{CO}_{2}$ for $16-24$ $\mathrm{h}$ before experimentation.

To perform siRNA transfections as previously described (Bone et al., 2016), custom synthesized siRNAs targeting specific transcripts with sequences as follows were obtained from Dharmacon (Lafayette, CO) as follows: non - targeting control: CGU ACU GCU UGC GAU ACG GUU (sense strand), and CGT ACT GCT TGC GAT ACG GUU (antisense strand); GSK3 $\beta$ : ACA CUA UAG UCG AGC CAA AUU (sense strand), and UUU GGC UCG ACU AUA GUG U (antisense strand); ULK1: GCA CAG AGA CCG UGG GCA AUU (sense strand), and UUG CCC ACG GUC UCU GUG CUU (antisense strand); APPL1: CAG AAU GUU CGC AGG GAA AUU (sense strand), and UUU CCC UGC GAA CAU UCU GUU (antisense strand), dynamin1, 5'-GGCUUACAUGAACACCAACCACGAA (Reis et al., 2015) (sense), CHC sequence, GGAAGGAAAUGCAGAAGAAUU (sense) (Garay et al., 2015), dynamin2, GGGCAGGCCUUCUAUAAGUUU (sense) (Garay et al., 2015), and PLC $\gamma 1$, GAGCAGUGCCUUUGAAGAAUU (sense) (Delos Santos et al., 2017). Cells were incubated with $220 \mathrm{pmol} / \mathrm{L}$ of each siRNA sequence with Lipofectamine RNAiMAX (LifeTechnologies) in Opti-MEM medium (Gibco) for 4 hours at 37C and 5\% CO 2. After this incubation period, cells 
were washed and incubated in fresh cell growth medium. siRNA transfections were performed twice, $72 \mathrm{~h}$ and $48 \mathrm{~h}$ prior to experiments.

\subsection{Whole -cell lysates, subcellular fractionation and Western blotting}

Western blotting using whole-cell lysates were performed as previously described (Garay et al., 2015). Cells were lysed in Laemmli sample buffer (LSB; 0.5 M Tris, pH 6.8, glycerol, 5\% bromophenol blue, 10\% $\beta$-mercaptoethanol, 10\% SDS; BioShop, Burlington, ON) containing phosphatase and protease cocktail ( $1 \mathrm{mM}$ sodium orthovanadate, $10 \mathrm{nM}$ okadaic acid, and $20 \mathrm{nM}$ protease inhibitor, all from BioShop, Burlington, ON). Cell Lysates were then heated to $65 \mathrm{C}$ for 15 min, then passed through with a 27.5 -gauge syringe. Proteins within whole -cell lysates were resolved by Glycine -Tris SDS -PAGE and then transferred onto a polyvinylidene fluoride (PVDF) membrane, which was then incubated with a solution containing specific primary antibodies. Western blot signal intensity detection corresponding to either phosphorylated proteins (e.g. pGSK3 $\beta$ S9), total proteins (e.g. GSK3 $\beta$ ), and the respective loading controls (e.g. actin) were obtained by signal integration in an area corresponding to the specific lane and band for each condition. The measurement of phosphorylation of a specific protein was obtained by normalization of the signal intensity of a phosphorylated form a protein to that of its loading control signal, then normalization to the signal intensity similarly obtained for the corresponding total protein. Similarly, as demonstrated in chapter 3, western blot signal intensity detection for phosphorylated Akt (eg. pAkt S473) normalized to total Akt (eg. Akt), then normalized to loading control (e.g. actin), which was designated as relative units (R.U.) (previously described 
by Garay et al., 2015). Measurements of the phosphorylated levels of other proteins were quantified similarly.

To examine phosphorylation of proteins for which no specific antibodies were available, we used the phos-tag gel system, which results in exaggeration of differences in apparent molecular weight of phosphorylated forms of specific proteins (Kinoshita et al., 2005). The phos -tag reagent was obtained from Wako (Osaka, Japan), and was used for conjugation within SDS PAGE polymerization as per the manufacturer's instructions. After SDS -PAGE was completed, gel was submerged in $\mathrm{MnCl}_{2}$ for chelation of remaining phos-tag moieties. Subsequently, protein intensity detection, measurement, and processing are identical to steps mentioned above. Subcellular fractionation to isolate nuclear and cytoplasmic fractions was done using the Nuclear Extraction Kit (cat \# ab219177 Abcam), as per the manufacturer's instructions.

\section{$\underline{2.5 \text { Immunofluorescence staining }}$}

Cells grown on glass coverslips were first subjected to fixation using cold methanol, blocked in 5\% bovine serum albumin (BioShop), then stained with specific primary antibodies, followed by appropriate fluorophore -conjugated secondary antibody and counter stained with DAPI. Lastly, cells were then mounted on glass slides in fluorescence mounting medium (DAKO, Carpinteria, CA). 


\subsection{Fluorescence microscopy}

Wide -field epifluorescence was performed on an Olympus IX83 Inverted Microscope with a 100x objective, coupled to a Hamamatsu ORCA -Flash4.0 digital camera (Olympus Canada, Richmond Hill, ON). Spinning disk confocal microscopy was performed using Quorum (Guelph, ON, Canada) Diskovery combination total internal reflection fluorescence and spinning -disc confocal microscope, operating in spinning disc confocal mode. This instrument is comprised of a Leica DMi8 microscope equipped with a $63 \times / 1.49$ NA objective with a $1.8 \times$ camera relay (total magnification 108×). Imaging was done using 488 -, 561 -, and 637 -nm laser illumination and 527/30,630/75, and 700/75 emission filters and acquired using a Zyla 4.2Plus sCMOS camera (Hamamatsu).

Structured illumination microscopy (SIM) was performed using a Zeiss Elyra PS.1 super -resolution inverted microscope, as previously described (Hua et al., 2017). Samples were imaged at an effective magnification of $101 x$ ( $63 \mathrm{x}$ objective $+1.6 \mathrm{x}$ optovar tube lens) on an oil immersion objective. Typically, 25 to 35 slices of $0.110 \mu \mathrm{m}$ were captured for each field of view for an imaging volume of approximately 2.75 to $3.85 \mu \mathrm{m}$. $488 \mathrm{~nm}, 561 \mathrm{~nm}$ and $643 \mathrm{~nm}$ laser lines were directed into the microscope optical train via a multimode fiber coupler. The lasers were passed through a diffraction grating, and a series of diffraction orders $(-1,0,+1)$ were projected onto the back focal plane of the objective. These wavefronts were collimated in the objective to create a three -dimensional sinusoidal illumination pattern on the sample. The diffraction grating was then rotated and translated throughout the acquisition to create patterned offset images containing encoded high spatial frequency information. Five lateral positions were 
acquired at each of five $\left(72^{\circ}\right)$ diffraction grating rotations for a total of 25 raw images per zplane. SIM imaging with all lasers was carried out at exposures varying from $50 \mathrm{~ms}$ to $100 \mathrm{~ms}$, with laser power varying between $3-10 \%(6-20 \mathrm{~mW}$ at the output), and a gain level of $60-80$. Imaging parameters were adjusted iteratively to achieve the best possible equalization of pixel intensity dynamic range across channels.

Raw SIM image stacks were processed in Zen under the Structured Illumination toolbar. A series of parameters were set to generate an optical transfer function (OTF) used for 3D reconstruction. The noise filter for Wiener de - convolution was set to a value of $1.0 \times 10-4$ to maximize the recovery of high spatial frequency information while minimizing illumination pattern artifacts. The maximum isotropy option was left unselected to recover all available frequency information at exactly the $72^{\circ}$ rotation angles. Negative values arising as an artifact of the Wiener filter were clipped to zero using the Baseline Cut option. Processed SIM images were then aligned via an affine transformation matrix of pre -defined values obtained using $100 \mathrm{~nm}$ multicolor Tetraspeck fluorescent microspheres (ThermoFisher Scientific).

\subsection{Fluorescence microscopy image analysis}

Measurement of total cellular signal intensity of specific proteins or GSK3 $\beta$ nuclear localization index were measured using ImageJ software (National Institutes of Health, Bethesda, MD). For total cellular measurements of specific protein signal, a region of interest corresponding to the cell outline, identified manually, was used to determine raw mean cellular fluorescence intensity. Final cellular signal intensity was obtained by subtracting background 
fluorescence (similarly obtained from a region on the coverslip with no cells) from the raw mean cellular fluorescence intensity, as previously described (Ross et al., 2015).

To determine GSK3 $\beta$ nuclear localization index, background -subtracted mean fluorescence intensity of regions of interest within the nucleus and cytoplasm were obtained. The GSK3 $\beta$ nuclear localization index is the ratio of these nuclear/cytosolic intensities. Each measurement was performed in at least three independent experiments, with $>30$ cells per condition, per experiment. Colocalization analysis was performed by determination of Manders' or Pearson's coefficients, as indicated, using the Just Another Colocalization Plugin (Bolte and Cordelieres, 2006) within ImageJ, as previously described (Bone et al., 2016).

Quantification of GSK3 $\beta$ localization in LAMP1-postitive membrane structures vs lysosome interior was performed (Fig. 6D) on 2 - colour 3D registered SIM images, which were split into single channel images consisting of GSK3 $\beta$ (red) or LAMP1 (green). To enhance the edge detection of LAMP1 -associated membrane structures, a Difference of Gaussians operation (DoG) was performed on the LAMP1 images (Marr and Hildreth, 1980) $(\sigma 1=1$ pixel, $\sigma 2=3$ pixels). The resulting bandpass -filtered image was used to trace a selection around the membrane by identifying edges via pixel intensity line profiles in ImageJ. The resulting selection was then superimposed onto the original, unfiltered image, and a binary image mask was generated encompassing only the selected pixels identified as membrane - associated LAMP1. A binary image of the corresponding GSK $3 \beta$ channel was created by thresholding via the auto threshold operation in ImageJ. The Minimum Cross Entropy thresholding method (Li and Tam, 1998) was selected as it minimized the unnecessary removal of data (false negatives) and the 
inclusion of spurious intensity values (false positives). The area of each feature (GSK3 $\beta$ puncta) in the image was measured and recorded. Subsequently, an image subtraction operation was performed by subtracting pixels corresponding to LAMP1 -positive membrane structures from the corresponding GSK3 $\beta$ channel. The area of the resulting GSK3 $\beta$ features was measured once again after the subtraction. Features for which a decrease in area was calculated were found to have pixels co - localizing with membrane -positive LAMP1, and thus assigned as membrane associated GSK3 $\beta$. Features for which the area remained constant were deemed to be associated with either the cytosol (outside the LAMP1 membrane selection) or the lysosomal interior (inside the LAMP1 membrane selection).

\subsection{Statistical analysis}

Statistical analysis was performed as previously described (Bone et al., 2017). Measurement of samples involving two experimental conditions (Figs. 4B, 6, S2, S3A \& S4B) were analyzed by student's t -test, with $\mathrm{p}<0.05$ as a threshold for statistically significant difference between conditions. Measurements of samples involving one experimental parameter and more than two conditions (Figs. 1, 2B - D, 4A, 4C, 5A \& S 1 D - E) were analyzed by one way ANOVA, followed by Bonferonni post -test to compare differences between conditions, with $\mathrm{p}<0.05$ as a threshold for statistically significant difference between conditions.

Measurements of samples involving two experimental parameters (Figures 3, 4D, 5 B, 7, S3B, \& S4D) were analyzed by two-way ANOVA, followed by Bonferonni post -test to compare differences between conditions, with $\mathrm{p}<0.05$ as a threshold for statistically significant difference between conditions. 
Chapter 3: Clathrin-dependent control of mitogenic signalling by EGFR 


\section{Rationale and background}

EGFR is activated by EGF, causing autotransphosphorylation, leading to phosphorylation of tyrosine residues in the c-terminal domain of the receptor (Lemmon et al., 2014; Honegger et al., 2006). These phosphorylated tyrosine residues are part of various motifs that additional proteins such as signalling scaffolds and signalling machinery, such as that leading to the activation of signalling pathways such as PI3K-Akt (Dibble and Cantley, 2015), PLC $\gamma 1$ (Nakamura and Fukami, 2017), and Erk (Morrison 2012).

These signals are orchestrated in a specific manner in space and time, such that maximal activation of Akt phosphorylation occurs after 1-5 min of EGF stimulation (Borisov et al., 2009). Within a few minutes of activation, EGFR undergoes CME, after which EGFR is either degraded or recycled back to the plasma membrane (Goh and Sorkin, 2013; Sorkin and Goh, 2009). Important for this work, the activation of signals by EGFR leading to activation of Akt signalling and the engagement of the CME protein and recruitment of EGFR into CCPs occurs simultaneously. As a result, much of the activation of signals by ligand-bound EGFR may occur while EGFR is located with CCPs.

For CME of receptors, the critical protein components clathrin and dynamin 2 are required at distinct stages of this process (Mettlen et al., 2013; Cocucci et al., 2012; McMahon and Boucrot, 2011; Taylor et al., 2011; Schmid and McMahon, 2007). Clathrin is absolutely required for the early stages of $\mathrm{CCP}$ formation, while dynamin is not required for the earliest stages but important for the very last stage of scission of CCPs from the plasma membrane to 
make vesicles (Kaksonen and Roux, 2018; McMahon et al., 2011). Dynamin has three known isoforms. While dynamin 2 has been well-described as the vesicle scission GTPase in nonneuronal cells, it has also been shown that dynamin 1 also participates in CME, highly expressed and studied in neuronal cells than non neuronal cells (Antonny et al., 2016). Recently, studies from the Schmid lab suggests that dynamin1 has yet unappreciated role in regulating certain aspects of CCP dynamics in non-neuronal cells (Chen et al., 2017; Reis et al., 2017; Reis et al., 2015), although much about this remains to be understood. It is possible that PLC $\gamma 1$ control of CCP occurs via engagement of dynamin1 because 1) PLC $\gamma 1$ can directly bind dynamin1 (Choi et al., 2004) and 2) PLC $\gamma 1$ signals to $\mathrm{Ca}^{2+}$, which can lead to dephosphorylation and thus activation of dynamin1 (Reis et al., 2017). If PLC $\gamma 1$ acts to control signalling via regulation of dynamin1, the phenotype of perturbation of dynamin 1 should be similar to that of perturbation of PLC $\gamma 1$ and/or clathrin.

Activated PLC $\gamma 1$, leads to the metabolism of important CME component, PI45P 2 (Nakamura and Fukami, 2017; Nishizuka 1988; Berridge and Irvine, 1984), into DAG and IP3, acting as secondary signals to regulate other downstream targets, including IP3 $\mathrm{Ca}^{2+}$ channels (Nakamura and Fukami, 2017; Nishizuka 1988; Berridge and Irvine, 1984). Activated IP3 $\mathrm{Ca}^{2+}$ channels activation results in the release of intracellular $\mathrm{Ca}^{2+}($ Nakamura and Fukami, 2017; Nishizuka 1988; Berridge and Irvine, 1984). In addition to clathrin and dynamin, which are required for the first and last stages of endocytosis, respectively, CME and the assembly of CCPs may also be regulated by PLC $\gamma 1$ and activation of $\mathrm{Ca}^{2+}$ derived signals. PLC $\gamma 1$ derived $\mathrm{Ca}^{2+}$ may regulate assembly of clathrin structures and can also regulate any aspect of cell function that depends on CCPs. 
Currently, as EGFR signalling occurs while the receptor is within CCPs, it is not understood whether spatial temporal regulation through internalization or receptor residence in CCP are required for EGFR signalling. Specifically, it is not well understood how CME components, clathrin and dynamin $1 / 2$, regulate EGFR activity to downstream signalling pathways. In other words, it is not known if the formation of CCPs (dependent on clathrin) or the scission of these CCPs from the plasma membrane (dependent on both clathrin and dynamin) is required to control EGFR signalling. Furthermore, how signalling pathways, such as PLC $\gamma 1$, regulate CME dynamics to regulate EGFR signalling and internalization, have yet to be elucidated.

Firstly, to understand how mitogenic signalling through EGFR is regulated by CME components, undertook characterization of the functional role of key CME components, clathrin and dynamin, in EGF-stimulated activation of Akt and Erk pathways. Secondly, examined how CME dynamics are modulated by PLC $\gamma 1$ activity, impacting the activation of Akt and Erk pathways. For this chapter, my working hypothesis is that clathrin acts as a scaffold to regulate EGF stimulated activation of the Akt pathway. Further, I hypothesize that regulation of clathrin assembly by PLC $\gamma 1$ is required for EGF-stimulated Akt activation. As a result, I look to explore the importance of clathrin nanodomains on plasma membrane EGFR signalling activity to downstream substrates. For this chapter, objectives include:

1) Determine how clathrin nanodomains affect Akt activity in MDA-MB-231 cells

2) Determine how dynamin1 affects Akt activity

3) Determine the affect of PLC $\gamma 1$ perturbations on Akt activity 


\subsection{Perturbation of clathrin, but not that of dynamin2, impaired Akt activation after EGF}

stimulation in MDA-MB-231 cells

Maximal Akt activation occurs within $~ 1-5$ minutes of EGFR ligand binding and activation (Borisov et al., 2009). Subsequently, Akt activation is attenuated, in part as the receptor undergoes membrane traffic after CME to early endosomes, leading to subsequent lysosome dependent degradation (Goh and Sorkin, 2013; Sorkin and Goh, 2009). It is not known if clathrin and/or dynamin are required for EGFR signalling lead to Akt activation.

To determine whether clathrin is required for Akt activation via EGF stimulation, I contributed to a team effort involving siRNA gene silencing of clathrin in ARPE-19 cells (henceforth, RPE cells). RPE cells were used as they contain low levels of ErbB2 (Garay et al., 2015). This work achieved an $80 \%(n=9)$ significant decrease in endogenous clathrin levels (Fig. 3.2C). All siRNA gene silencing knockdown levels described in this chapter were previously demonstrated (Garay et al., 2015). Silencing of clathrin lead to a significant decrease in EGF-stimulated Akt phosphorylation on S473 over the entire 15 min observation time period (Fig. 3.1A\&C, $\mathrm{n}=12$; individual experimental repeats were generated in collaboration with Camillo Garay, Gurjeet Judge, Rohan Pandey and Stefanie Lucarelli). To understand whether clathrin is required for EGFR signalling as a plasma membrane scaffold or if EGFR signalling to Akt requires receptor internalization, we also performed siRNA gene silencing of dynamin2. As noted above, dynamin 2 is required for the scission of clathrin coated pits and subsequent internalization, but not for the formation of plasma membrane clathrin scaffolds (CCPs). Importantly, silencing of dynamin2 did not affect EGF-stimulated Akt phosphorylation over a 
15-min observation time period (Fig. 3.1 A\&C, $\mathrm{n}=7$; individual experimental repeats where generated in collaboration with Camillo Garay, Gurjeet Judge, Rohan Pandey and Stefanie Lucarelli). Notably, work from collaborators on this project revealed that clathrin perturbation impacted signalling downstream of EGFR phosphorylation, but did not affect EGFR ligand binding or autophosphorylation on a number of residues (Appendix Fig. A1). Furthermore, since clathrin and dynamin2 levels may control the turnover of EGFR, levels of EGFR were not affected when treated with siRNA gene silencing against clathrin and dynamin2 as previously demonstrated (Garay et al., 2015). These results suggest that receptor residence in clathrin coated pits, but not receptor internalization, is required for Akt activation via EGFR ligand stimulation in RPE cells (a non-cancerous retinal epithelial pigment epithelial cell line).

To determine if clathrin, but not receptor endocytosis, is also required for EGF-stimulated Akt phosphorylation is also required in triple-negative breast cancer cells, I examined this phenomenon in MDA-MB-231 cells. Similar to RPE cells, MDA-MB-231 cells were used as they also contain low levels of ErbB2 (Garay et al., 2015). In MDA-MB-231 cells, EGFR is strongly recruited to clathrin coated pits following EGF stimulation and EGFR endocytosis is sensitive to dynamin inhibition by dynasore (Mutch et al., 2014). Therefore, EGFR internalization is strongly dynamin dependent in these cells. MDA-MB-231 cells treated with 10 $\mu \mathrm{M}$ of clathrin inhibitor, pitstop2, leads to the significant decrease in Akt S473 phosphorylation after EGF stimulation (Fig. 3.2.A\&B). In contrast, MDA-MB-231 cells treated with $80 \mu \mathrm{M}$ of dynamin2 inhibitor dynasore, exhibited normal Akt S473 phosphorylation after EGF stimulation (Fig. 3.2A\&B). These results are consistent with observations in RPE cells, and again suggest that clathrin, but not dynamin2 and receptor endocytosis, is required for EGF-stimulated Akt 
phosphorylation in MBA-MB-231 cells. Complementing drug experiments, siRNA silencing of clathrin in MDA-MB-231 cells also significantly perturbed EGF-stimulated Akt activation (Fig. 3.2C\&D). Collectively, these results show that similarly as in RPE cells, in MDA-MB-231 cells, clathrin is required to regulated Akt activation upon EGFR activation, and that this requirement for clathrin may reflect a role for clathrin to form signalling nanodomains or scaffolds at the cell surface, and not a role for clathrin for leading to eventual dynamin-dependent internalization of EGFR.

Other results from Garay et al., 2015 demonstrated that perturbations in clathrin inhibited EGF stimulated Gab1 phosphorylation (Garay et al., 2015). Furthermore, phosphorylated Gab1 and Gab1 are enriched in CCPs (Garay et al., 2015). This provides support that CCPs are important nanodomains at the cell surface.

3.2 PLC $\gamma 1$ affects CME dynamics and Akt phosphorylation but not Erk phosphorylation

EGFR activation leads to triggering of the PLC $\gamma 1$ signalling pathway (Nakamura and Fukami, 2017; Nishizuka 1988; Berridge and Irvine, 1984). Once activated, PLC $\gamma 1$, metabolizes $\mathrm{PI}(4,5) \mathrm{P}_{2}$ leading to the generation of DAG and IP3, the latter which is a secondary messenger that triggers $\mathrm{Ca}^{2+}$ signalling (Nakamura and Fukami, 2017; Nishizuka 1988; Berridge and Irvine, 1984). How PLC $\gamma 1$ signalling may be required for the regulation of CCP formation, in turn impacting EGFR signalling leading to Akt and Erk phosphorylation, is not well understood. 
As per Delos Santos et al., 2017, perturbations of PLC $\gamma 1$ leads to a decrease in assembly of CCPs that contain EGFR, and CME mediated EGFR internalization (Delos Santos et al., 2017). This suggests that EGF-stimulated PLC $\gamma 1$ signals are required for clathrin assembly. Since we had also shown that clathrin perturbations leads to a decrease in EGF stimulate Akt phosphorylation, this suggested that EGF-stimulated PLC $\gamma 1$ signals may impact clathrindependent Akt phosphorylation. To test this, I used siRNA gene silencing of PLC $\gamma 1$, which lead to an $85.6 \pm 6.4 \%$ reduction $(\mathrm{p}<0.05, \mathrm{n}=3)($ Fig. 3.3A) in expression levels PLC $\gamma 1$. Importantly, PLC $\gamma 1$ silencing resulted in a robust reduction in EGF stimulated Gab1 and Akt phosphorylation (Fig. 3.3B). In contrast, and similar to the effect of clathrin perturbation, PLC $\gamma 1$ silencing did not affect EGFR phosphorylation (Fig. 3.3B\&C), nor EGF-stimulated Erk phosphorylation, (Fig. 3B, $\mathrm{n}=3, \mathrm{n}<0.05$ ). As previously described by Delos Santos et al., in 2017, perturbations in PLC $\gamma 1$ signalling negatively impacts the initiation and assembly of CCPs, which contain EGFR (Delos Santos et al., 2017). These results are consistent with PLC $\gamma 1$ regulating EGFR residence in CCPs and internalization, suggesting that PLC $\gamma 1$ plays a role in regulating CME dynamics to control specifically the EGFR-Gab1-Akt pathway.

\subsection{Dynamin 1 perturbations affects EGFR and Akt phosphorylation but Erk activity}

PLC $\gamma 1$ has been reported to control dynamin1 controlling guanyl exchange as a GEF in neuronal cells (Choi et al., 2004). In turn, dynamin1 has also been reported to control tumor necrosis factor tumor necrosis factor related apoptosis induce ligand (TRAIL) death receptor is regulated via CME through dynamin1 (Reis et al., 2017). Although dynamin2 is not required for EGF stimulated Akt activity, it is not well understood how the dynamin1 isoform may contribute 
to EGFR internalization and/or signalling. Using siRNA gene silencing to dynamin1, endogenous levels of dynamin1 was significantly decreased to $80 \%\left(n=3,{ }^{*} \mathrm{p}<0.05\right)$ (Fig. 3.4A). Interestingly, siRNA silencing of dynamin1, perturbed both EGFR and Akt phosphorylation, but not that of Erk (Fig. 3.4 B\&C, $n=3,{ }^{*} p<0.05$ ). In collaboration with Dr. R.C. Delos Santos, siRNA of PLC $\gamma 1$ perturbs EGF internalization and increases cell surface EGFR levels (Appendix Fig. A2.A). Furthermore, Dr. R.C. Delos Santos demonstrated that dynamin1 siRNA does not alter EGFR internalization but decreases cell surface levels of EGFR (Appendix Fig. A2.B). This suggests that both dynamin1 and PLC $\gamma 1$ regulate EGFR signalling in two separate pathways due to two different phenotypes. Thus, it is unlikely that PLC $\gamma 1$-calcium is acting through regulation via activation of dynamin1 to regulate EGFR signalling. It is possible that dynamin1 may not have a direct role in regulating signalling, but rather to promote recycling over degradation, which is consistent with a study conducted by Lakoduk et al., in 2019 (Lakoduk et al., 2019).

In summary, EGFR residence in CCPs (clathrin) and not receptor internalization (dynamin2) is required for Gab1 and Akt activation in RPE and triple negative breast cancer (MDA-MB-231) cells. Activation of the Erk pathway does not require EGFR CCP residence or receptor internalization. Additionally, PLC $\gamma 1$ modulates CME dynamics controlling Gab1 and Akt activation. Furthermore, dynamin1 may not be regulated by PLC $\gamma 1$ activity, but both may work in two different pathways to regulate EGFR activity, where dynamin1 regulates EGFR recycling rather than direct signalling. 

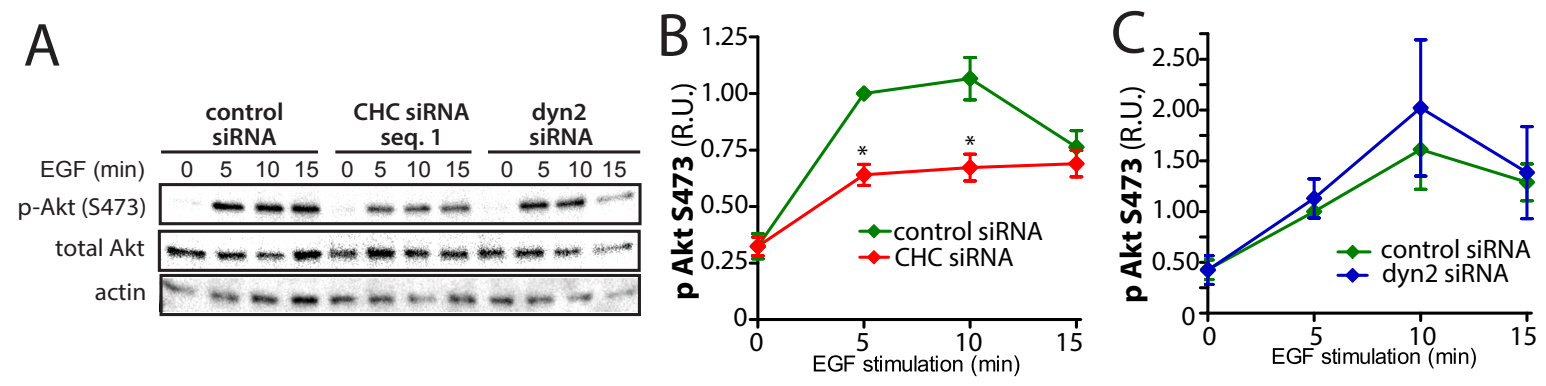

Figure 3. 1 siRNA gene silencing of clathrin and not dynamin2 perturb Akt

phosphorylation after EGF stimulation in ARPE-19 cells. ARPE-19 cells were transfected using siRNA against nontargeting siRNA (control), clathrin (CHC), or dynamin2 (dyn2). (A) After siRNA transfection, ARPE-19 cells were stimulated EGF $(5 \mathrm{ng} / \mathrm{ml})$ or unstimulated (basal). Shown are representative immunoblot images of whole-cell lysates probed for antipSer473-Akt, anti-total Akt, and anti-total actin antibodies. (B-C) Shown are mean \pm SE of antipSer473-Akt values; $n=12$ (B) $n=7(C) ;{ }^{*} p<0.05$ relative to control to condition EGFstimulated. 

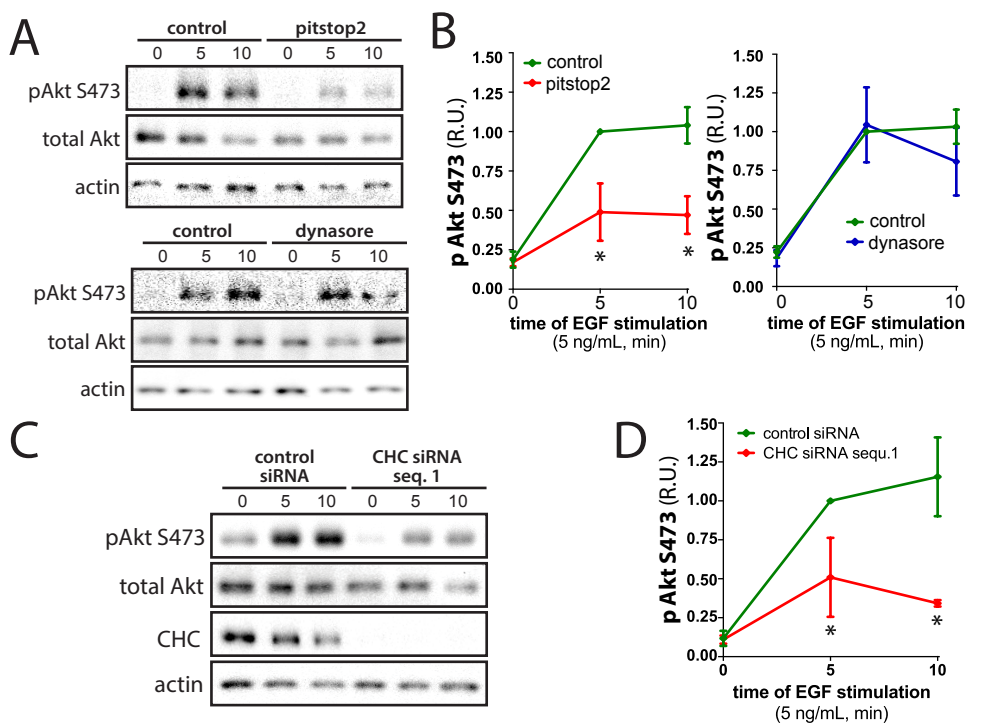

Figure 3.2 Clathrin perturbations inhibit Akt phosphorylation after EGF stimulation in MDA-MB-231 cells. (A-B) MDA-MB-231 cells were treated pitstop $(10 \mu \mathrm{M})$, dynasore $(80$ $\mu \mathrm{M})$, or vehicle control $(0.1 \%$ [vol/vol] DMSO) for 20 minutes. (C-D) MDA-MB-231 cells were transfected using siRNA against nontargeting siRNA (control) or clathrin (CHC). After drug treatment or siRNA gene silencing, MDA-MB-231 cells were stimulated with $(5 \mathrm{ng} / \mathrm{ml})$ or unstimulated (basal). (A \& C) Shown are representative immunoblot images of whole-cell lysates probed for anti-pSer473-Akt, anti-total Akt, anti-total actin and anti-total CHC antibodies. (B \& D) Shown are mean \pm SE of anti-pSer473-Akt values; $(\mathrm{n}<3) * p<0.05$ relative to control condition EGF-stimulated. 

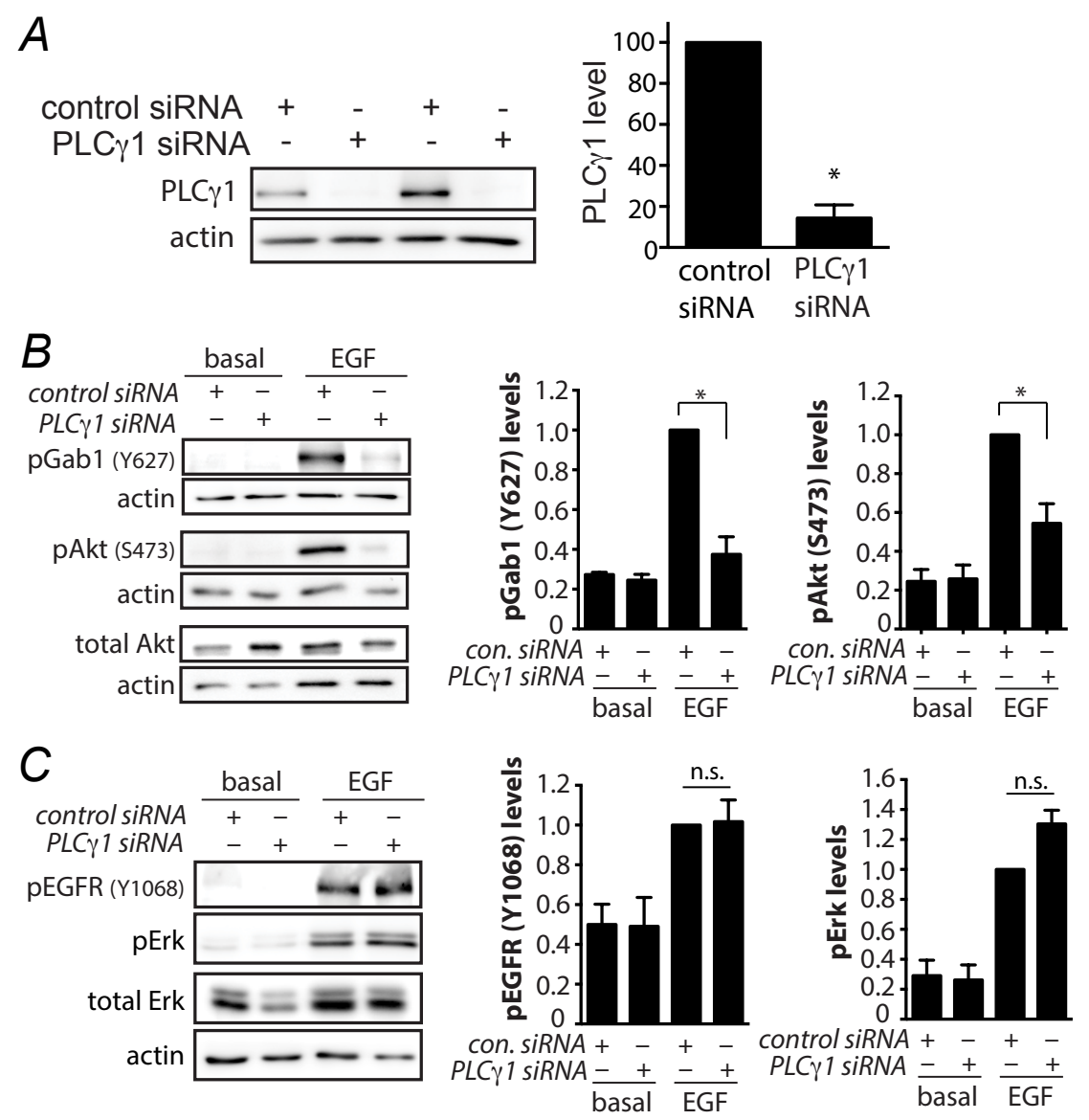

Figure 3. 3 PLC $\gamma 1$ perturbations affect CME dynamics inhibiting EGF stimulated Akt phosphorylation. (A-C) ARPE-19 cells were transfected using siRNA against nontargeting siRNA (control) and PLC $\gamma 1$. (B\&C) After siRNA transfection, ARPE-19 cells were stimulated EGF $(5 \mathrm{ng} / \mathrm{ml}$ ) for 5 minutes or unstimulated (basal). (A-C) Shown are representative immunoblot images of whole-cell lysates probed for anti-total- PLC $\gamma 1$, anti-pTyr627-Gab1, antipSer473-Akt, anti-total Akt, anti-pTyr1068-EGFR, anti-pThr202/Tyr204-Erk, anti-total-Erk and anti-total actin antibodies. (A-C) (A) Shown are mean \pm SE of anti-total- PLC $\gamma 1$ values; $(n=3)$; $*_{p}<0.05$ relative to control condition (control siRNA). (B-C) Shown are mean \pm SE of antipTyr307-Gab1, pSer473-Akt, pTyr1068-EGFR, and pThr202/Tyr204-Erk values; $\mathrm{n}=3$; * $p$ $<0.05$ relative to siRNA control condition EGF-stimulated. 


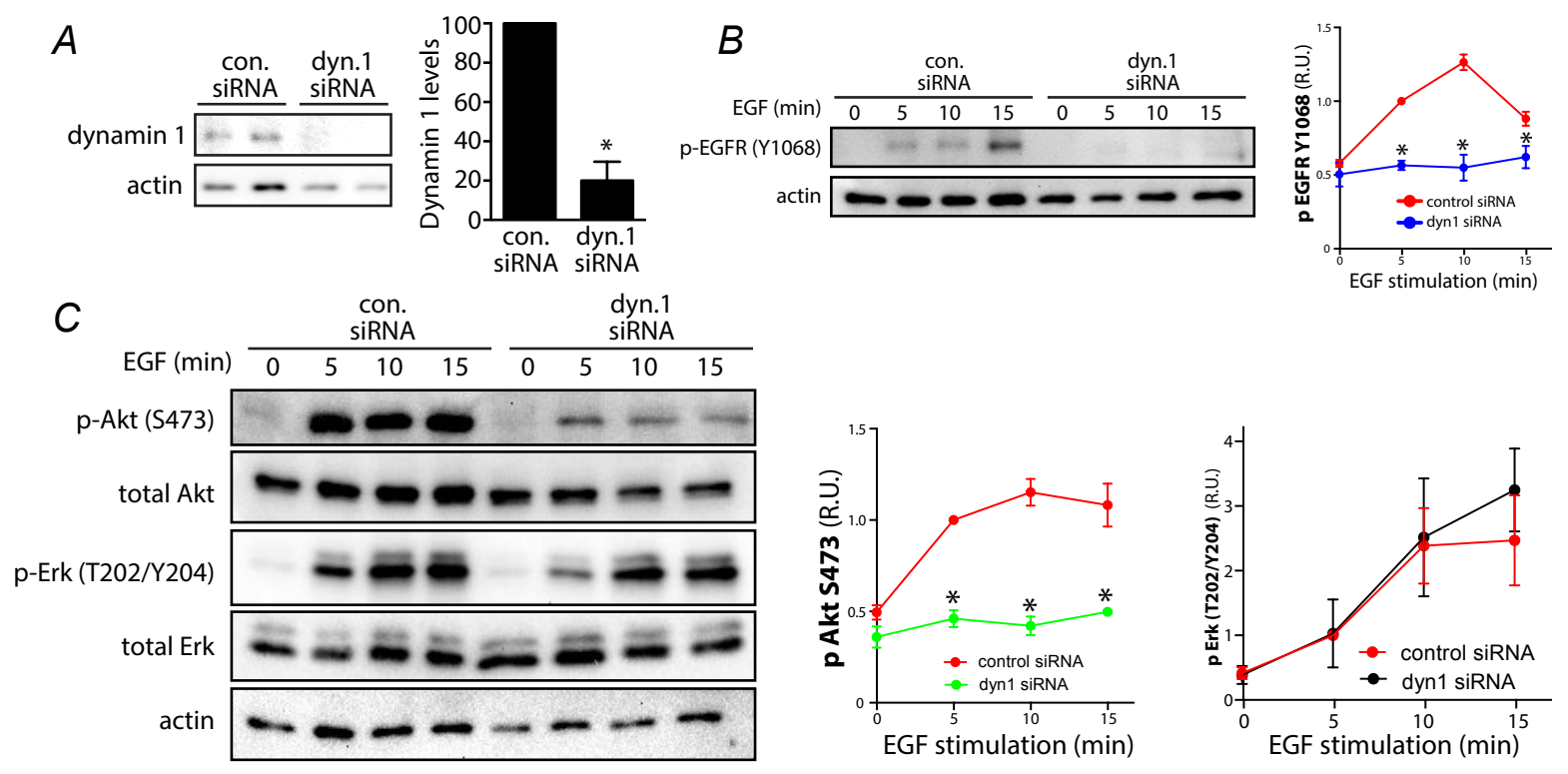

\section{Figure 3. 4 Dynamin1 knockdown impairs EGF stimulated EGFR and Akt}

phosphorylation. (A-C) ARPE-19 cells were transfected using siRNA against nontargeting siRNA (control) and dynamin1. (B\&C) After siRNA, transfection, ARPE-19 cells were stimulated with $(5 \mathrm{ng} / \mathrm{ml})$ for 15 minutes or unstimulated (basal). Shown are representative immunoblot images of whole-cell lysates probed for anti-total dynamin1, anti-pTyr1068EGFR, anti-pSer473-Akt, anti-total Akt, anti-pThr202/Tyr204-Erk, anti-total-Erk and antitotal actin antibodies. (A-C) (A) Shown are mean \pm SE of anti-total-dynamin 1 values; $(n=3)$; $*_{p}<0,05$ relative to control condition (control siRNA). (B-C) Shown are mean $\pm \mathrm{SE}$ of pTyr1068-EGFR, pSer473-Akt and pThr202/Tyr204-Erk values; $\mathrm{n}=3 ;{ }^{*} p<0.05$ relative to siRNA control condition EGF-stimulated. 
Chapter 4: GSK3 localization and activity 


\section{Background and Rationale}

The kinase activity of GSK3 (I will be examining primarily GSK $\beta$, but will examine similarities to GSK $3 \alpha$ ) is regulated by many signalling pathways including direct

phosphorylation by Akt, PKC, and p90RSK (Frame et al., 2001). After EGF stimulation, EGFR activates PI3K-Akt pathway, leading to direct phosphorylation of Serine 9 on GSK $\beta$ by Akt, subsequently inhibiting kinase activity (Frame et al., 2001). In addition to this S9 phosphorylation regulating GSK $\beta$ activity, control of subcellular localization may be a key mechanism to gate GSK $\beta$ activity, as many GSK $\beta$ substrates are selectively localized to different membrane compartments within the cells (Reis et al., 2015; Chiara et al., 2013; Wu and Pan, 2010; Schenck et al., 2008; Zeng et al., 2008; Hoshi et al., 1996).

GSK $\beta$ regulates different downstream substrates such as transcription factors, many of which are localized within the nucleus, including c-Myc (Gregory et al., 2003; Manoukian and Woodgett, 2002; Sears et al., 2000) and Snail (Zhou et al., 2004). c-Myc and Snail are phosphorylated by GSK $\beta$, promoting their proteosomal degradation (Zhou et al., 2004). Thus, in order for GSK $\beta$ to regulate c-Myc and Snail, there is a requirement for certain signals to modulate GSK $\beta$ to ensure localization to the nucleus.

mTORC1 is a master regulator as it integrates both mitogenic and metabolic signalling (Saxton and Sabatini, 2017). Mitogenic signalling such as PI3K-Akt activates mTORC1 activity via control of the TSC 1/2 complex, which has an outcome to enhance Rheb activity at the 
lysosome to activate mTORC1 (Saxton and Sabatini, 2017). Additionally, metabolic signalling largely regulates mTORC1 activity controlled by amino acid abundance within the cell sensed by V-ATPase and other amino acid sensors, which has an outcome to increase GTP binding of Rag GTPases, which in turn leads to enhanced mTORC1 recruitment to the lysosome where it can be activated by Rheb (Bar-Peled et al., 2012; Zoncu et al., 2011). Furthermore, mTORC1 indirectly senses energy changes in ATP levels through energy sensor AMPK (Gwinn et al., 2008; Shaw et al., 2004; Inoki et al., 2003). Thus, the activity of mTORC1 is regulated in a manner that integrates both mitogenic and metabolic signalling, such that both arms of signalling are required to ensure mTORC1 activity.

Currently, it is well established that the PI3K-Akt pathway plays a role in regulation of GSK $\beta$ as Akt directly phosphorylates (S9) and inhibits GSK $\beta$ activity. How this signalling pathway may also impact GSK $\beta$ localization remains poorly understood. It is possible that Aktmediated S9 phosphorylation of GSK $\beta$ may control GSK $\beta$ localization. Alternatively, it is also possible that PI3K-Akt signalling may indirectly control GSK $\beta$ localization, through engagement of Akt downstream substrates, such as mTORC1. Furthermore, it is not well understood how both mitogenic and metabolic signalling regulate mTORC1 activity towards GSK $\beta$ localization and activity, and how this may impact GSK $\beta$ control of cell physiology under distinct cellular and environmental conditions.

To understand how signals within the PI3K-Akt-mTORC1 signalling pathway control GSK $\beta$ activity and localization, I examined PI3K-Akt-mTORC1 signals and how these contribute to regulating the ability of GSK3 to control expression levels of c-Myc and Snail. As 
c-Myc and Snail are restricted to the nucleus, regulation of their expression mediated by GSK $\beta$ phosphorylation requires GSK $\beta$ localization to the nucleus. Next, I resolved how both mitogenic (PI3K-Akt) and metabolic (amino acids and ATP availability signalling) pathways that control mTORC1 activity, regulate GSK $\beta$ nucleocytoplasmic shuttling. Finally, I examined how phosphorylation of serine 9 impacts GSK $\beta$ localization. My working hypothesis for this chapter was that mTORC1 signalling regulates GSK $\beta$ nucleocytoplasmic shuttling and thus controls GSK $\beta$ activity towards nuclear substrates. For this chapter, the objectives were to:

1) Determine how mitogenic signalling affects GSK $\beta$ activity

2) Determine how mitogenic and metabolic signalling affects GSK $\beta$ localization

3) Determine the effect of phosphorylation of GSK $\beta$ localization

\section{$\underline{4.1 \text { mTORC } 1 \text { controls GSK3} 3 \beta \text { nuclear localization and c-Myc levels }}$}

mTORC1 I known to regulate c-Myc levels but whether there is post translational regulation, such as by control of c-Myc turnover by mTORC1, is not known. Treatment of RPE cells with $1 \mu \mathrm{g} / \mathrm{mL}$ rapamycin caused a time-dependent decrease in c-Myc levels, reaching $57 \pm$ $4.8 \%$ after 2 hours of rapamycin treatment $(n=6, p<0.05$, Fig. 4.1A). Importantly, co-treatment with $10 \mu \mathrm{M}$ of the GSK3 $\beta$ kinase inhibitor CHIR99021 blunted the decrease in c-Myc levels elicited by rapamycin treatment (Fig. 4.1A). Consistent with this result, rapamycin treatment also elicited a reduction in levels of the transcription factor Snail, an effect also blunted by cotreatment with CHIR99021 (Fig. 4.1B). 
I next used siRNA gene silencing of GSK3 $\beta$, which resulted in a $91 \pm 4.7 \%$ reduction of GSK3 $\beta$ protein levels $(n=3, p<0.05$, Fig. 4.2A). While RPE cells also express the paralog GSK3 $\alpha$, silencing of GSK3 $\beta$ was specific and did not impact expression of GSK3 $\alpha$ (Fig. 4.2B). Cells subjected to silencing of GSK3 $\beta$ exhibited no change in c-Myc expression level upon inhibition of sequential signals in the PI3K-Akt-mTORC1 axis, achieved by treatment with either LY294002, Akti-1/2, or rapamycin, respectively (Fig. 4.1C). In contrast, each inhibitor effectively reduced c-Myc levels in cells subjected to non-targeting (control) siRNA treatment (Fig. 4.1C). Taken together, these results indicate that PI3K-Akt signals converge on mTORC1 to enhance c-Myc levels in a manner that requires the regulation of GSK3 $\beta$.

To determine how PI3K-Akt-mTORC1 signals control c-Myc levels in a GSK3 $\beta$ dependent manner, we examined the localization and levels of endogenous GSK3 $\beta$ and c-Myc. Consistent with previous reports (Bechard and Dalton, 2009; Meares and Jope, 2007; Zmijewski and Jope, 2004; Bijur and Jope, 2001; Diehl et al., 1998), in cells grown in serum (e.g. with an active PI3K-Akt-mTORC1 axis), GSK3 $\beta$ primarily localizes within the cytosol and appears mostly excluded from the nucleus (Fig. 4.3A). We confirmed the specificity of detection of endogenous GSK3 $\beta$ by immunofluorescence microscopy following GSK3 $\beta$ silencing (Fig. 4.2C). In contrast, and as expected (Smith et al., 2004; Hann et al., 1983; Abrams et al., 1982), cMyc localizes virtually entirely within the nucleus under these conditions (Fig. 4.3A). Thus, under conditions in which mTORC1 is active, GSK3 $\beta$ and c-Myc are compartmentalized separately within the cytosol and nucleus, respectively. 
I next determined how PI3K-Akt-mTORC1 signalling regulates GSK3 $\beta$ localization. Treatment of RPE cells with either LY294002, Akti-1/2, or rapamycin to perturb PI3K, Akt or mTORC1, respectively resulted in robust and significant $(n=3, p<0.05)$ increase in nuclear GSK $3 \beta$, measured by the ratio of nuclear to cytosolic mean fluorescence intensities of GSK3 $\beta$ which I term the GSK3 $\beta$ nuclear localization index (Chapter 2: Fluorescence microscopy image analysis) (Fig. 4.3B). Importantly, the effect of rapamycin treatment on GSK3 $\beta$ nuclear translocation and Snail protein levels was also observed in MDA-MB-231 breast cancer cells (Fig. 4.2D-E), demonstrating that the mTORC1- dependent control of GSK3 $\beta$ is not unique to RPE cells. Similar observations were made when detecting GSK3 $\beta$ cytoplasmic and nuclear localization by subcellular fractionation (Fig. 4.3C). Furthermore, inhibition of the PI3K-AktmTORC1 axis also resulted in robust nuclear localization of GSK3 $\alpha$ (Fig. 4.3D), a paralog of GSK3 $\beta$ with highly similar kinase domains but unique terminal motifs (Cormier and Woodgett, 2017; Woodgett 1990). These results indicate that PI3K-Akt signals act via control of mTORC1 to regulate GSK3 $\beta$ nuclear localization, as well as that of GSK3 $\alpha$.

Ran is a GTPase that is required for the nuclear export cargo proteins (Kim et al., 2017; Wente and Rout, 2010; Kau et al., 2004). To test the importance of Ran in mTORC1-dependent GSK3 $\beta$ nuclear translocation, I examined the impact of Ran GTP-binding mutants on GSK3 $\beta$ localization. I expressed wild type (WT) Ran or one of two Ran mutants, Ran T24N and G19V, which are constitutively GDP- or GTP-bound, respectively (Carey et al., 1996). Ran T24N is a constitutively GDP-bound, should allow nuclear import but not nuclear export, which should promote nuclear import of cargo proteins irrespective of other signals (Carey et al., 1996). In contrast, Ran G19V, which is GTP-bound, should allow nuclear export but not nuclear import 
(Carey et al., 1996). Cells expressing WT Ran exhibited little nuclear GSK3 $\beta$ in the control condition, but a robust localization of GSK3 $\beta$ in the nucleus was observed upon treatment with rapamycin (Fig. 4.4, upper panels, and quantification, lower panel). In contrast, cells expressing Ran T24N exhibited nuclear GSK3 $\beta$ in both control and rapamycin-treated conditions (Fig. 4.4), consistent with Ran-GDP acting to facilitate nuclear import (Carey et al., 1996).

Further, cells expressing Ran G19V exhibited mostly cytosolic GSK3 $\beta$ in both control and rapamycin-treated conditions, consistent with this mutant blocking Ran-dependent nuclear import (Fig. 4.4). This makes sense since, Ran G19V is constitutively GTP-bound, which does promote nuclear export of cargo (Carey et al., 1996). These experiments confirm that the change in localization of GSK3 $\beta$ observed upon treatment with rapamycin indeed corresponds to nuclear translocation, mediated by canonical Ran-dependent nuclear import via the NPC. Further, these results indicate that GSK3 $\beta$ undergoes Ran-dependent nucleocytoplasmic shuttling and Randependent nuclear import that is regulated by mTORC1.

\subsection{Metabolic cues regulate GSK3 $\beta$ nuclear localization via mTORC1}

As mTORC1 is regulated by both mitogenic (PI3K-Akt) signals as well as metabolic cues, in this section I examined how metabolic signals contribute to the control of GSK3 $\beta$ nuclear localization. AMPK is activated via ATP insufficiency, and negatively regulates mTORC1 signalling through phosphorylation and activation of TSC2 (Shaw et al., 2004, Inoki et al., 2006). Consistent with the effects of mTORC1 inhibition by rapamycin, treatment with the AMPK activator A769662 resulted in robust GSK3 $\beta$ nuclear localization (Fig. 4.5A). 
Importantly, AMPK and mTORC1 exhibit reciprocal negative regulation (Inoki et al., 2012). As such, GSK3 $\beta$ nuclear localization could conceivably be the direct result of loss of mTORC1 activity, or an increase in AMPK activation, both of which would be expected to occur upon treatment with either rapamycin or A769662. To dissect a role for mTORC1 versus AMPK in control of GSK3 $\beta$ nuclear localization, we used the AMPK inhibitor compound C (Ross et al., 2012). Cells treated with compound $C$ exhibited a rapamycin-dependent increase in GSK3 $\beta$ nuclear localization comparable to that observed in cells treated with rapamycin but not compound C (Fig. 4.5A). This indicates that AMPK activity is dispensable for GSK3 $\beta$ nuclear localization induced by mTORC1 inhibition. As GSK3 $\beta$ forms a complex with AMPK (Suzuki et al., 2013), we also tested whether AMPK may have a kinase-independent, structural role in regulation of GSK3 $\beta$. However, silencing of AMPK did not impact GSK3 $\beta$ nuclear localization (Fig. 4.6). Collectively, these results indicate that while AMPK activation also triggers an accumulation of nuclear GSK3 $\beta$, this occurs as a result of AMPK-dependent inhibition of mTORC1 signals, and not as a result of direct action of AMPK on GSK3 $\beta$ nuclear localization.

mTORC1 is activated by abundance of amino acids in a manner that requires the VATPase (Zoncu et al., 2011). To determine how amino acid-dependent activation of mTORC1 impacted control of GSK3 $\beta$ localization, we treated cells with the V-ATPase inhibitor concanamycin A. Cells treated with concanamycin A exhibited a significant enhancement of nuclear GSK3 $\beta$ relative to control (Fig. 4.5B). Consistent with this result, amino acid deprivation achieved via incubation of cells in amino acid and glucose depleted media (EBSS) also mimicked the effect of rapamycin treatment in RPE (Fig. 4.5C) as well as MDA-MB-231 (Fig. 
4.2D) cells. These results indicate that amino acid sensing by mTORC1 contributes to the regulation of GSK3 $\beta$ nuclear localization.

mTORC1 inhibition also leads to induction of autophagy (Jung et al., 2009), and it was thus important to consider the possibility that GSK3$\beta$ was undergoing nuclear translocation in response to induction of autophagy or in response to autophagy-induction signalling. We therefore tested whether autophagy is required for GSK3 $\beta$ nuclear localization upon mTORC1 inhibition with rapamycin. To inhibit one of the earliest stages of signalling leading to autophagy induction, we treated cells siRNA targeting endogenous ULK (Saric et al., 2016), which resulted in a robust $77 \% \pm 6.2$ reduction of ULK expression $(n=3, p<0.05$, Fig. $4.7 A)$. Cells treated with siRNA to silence ULK1 exhibited cytosolic GSK3 $\beta$, which relocalized to the nucleus upon rapamycin treatment in a manner indistinguishable from cells treated with non-targeting siRNA (Fig. 4.5D). As autophagy induction has also been reported to lead to c-Myc degradation (Cianfanelli et a., 2015), we also tested the effect of ULK1 silencing on rapamycin-induced cMyc levels. Surprisingly, silencing of ULK1 on its own reduced c-Myc levels (Fig. 4.7D). Moreover, and in contrast to the findings of a previous study (Cianfanelli et a., 2015), impairment of autophagy induction by ULK1 silencing did not prevent the rapamycin-induced reduction in c-Myc levels (Fig. 4.7B). Thus, GSK3 $\beta$ nuclear translocation and c-Myc degradation observed upon mTORC1 inhibition are largely independent of autophagy induction. Instead, c-Myc degradation upon mTORC1 inhibition is mediated by regulation of GSK3 $\beta$ nuclear localization and function. 


\section{$\underline{4.3 \text { Control of GSK } 3 \beta \text { nuclear localization does not require GSK3 } \beta \text { S9 phosphorylation }}$}

Akt phosphorylates GSK3 $\beta$ on S9, which negatively regulates GSK3 $\beta$ kinase activity towards certain substrates. We next examined how GSK3$\beta$ phosphorylation may contribute to control of GSK3 $\beta$ nuclear localization by mTORC1. As expected, cells treated with LY294002 or Akti-1/2 exhibited significant reduction in GSK3 $\beta$ S9 phosphorylation by $80 \pm 0.8 \%$ and $60 \pm$ $6.8 \%$ respectively $(n=3, p<0.05$, Fig. $4.6 A)$. In contrast, cells treated with rapamycin exhibited no change in GSK3 $\beta$ S9 phosphorylation compared to control (Fig. 4.6A). These results uncouple S9 phosphorylation from control of GSK3 $\beta$ nuclear localization. To directly probe the contribution of GSK3 $\beta$ S9 phosphorylation to mTORC1-dependent GSK3 $\beta$ nuclear localization, we studied the subcellular localization of a S9A mutant of GSK3 $\beta$. I expressed either wild-type or S9A GSK3 $\beta$ in cells, and I was able to selectively detect the exogenous GSK3 $\beta$ via an HAtag. GSK3 $\beta$ S9A is a point mutation of GSK3 $\beta$ that impairs S9 phosphorylation from upstream regulators, preventing inhibition (Stambolic and Woodgett, 1994). Under basal conditions, GSK3 $\beta$ S9A remains cytosolic, while treatment with the Akt inhibitor Akti-1/2 resulted in nuclear localization of GSK3 $\beta$ S9A, as seen with GSK3 $\beta$ WT (Fig. 4.6B).

Using phos-tag acrylamide electrophoresis, a technique that exaggerates differences in apparent molecular weight of phosphorylated species of a protein (Kinoshita et al., 2006), we

observed two detectable species of GSK3 $\beta$, of which the higher molecular weight species likely corresponds to the S9 phosphorylated form given its sensitivity to PI3K and Akt inhibition (Fig. 4.9). In contrast and as expected, rapamycin had no effect on GSK3 $\beta$ detected by this method. Collectively, these results indicate that regulation of GSK3 $\beta$ S9 phosphorylation does not 
contribute to control of GSK3 $\beta$ nuclear localization by PI3K-Akt-mTORC1 signals. These phostag gel experiments also suggest that it is unlikely that additional signals.

In summary, signals that control mTORC1 activity including mitogenic (PI3K-Akt) and metabolic (AMPK and Amino acids availability), regulate GSK3 $\beta$ nuclear translocation and activity in RPE and MDA-MB-231 cells. GSK3 $\beta$ undergoes Ran GTPase dependent nuclear translocation and does not require serine 9 phosphorylation to translocate into the nucleus. As a result of mTORC1 dependent control of GSK3 3 nuclear translocation, GSK3 $\beta$ targets effector proteins c-Myc and Snail for degradation. 

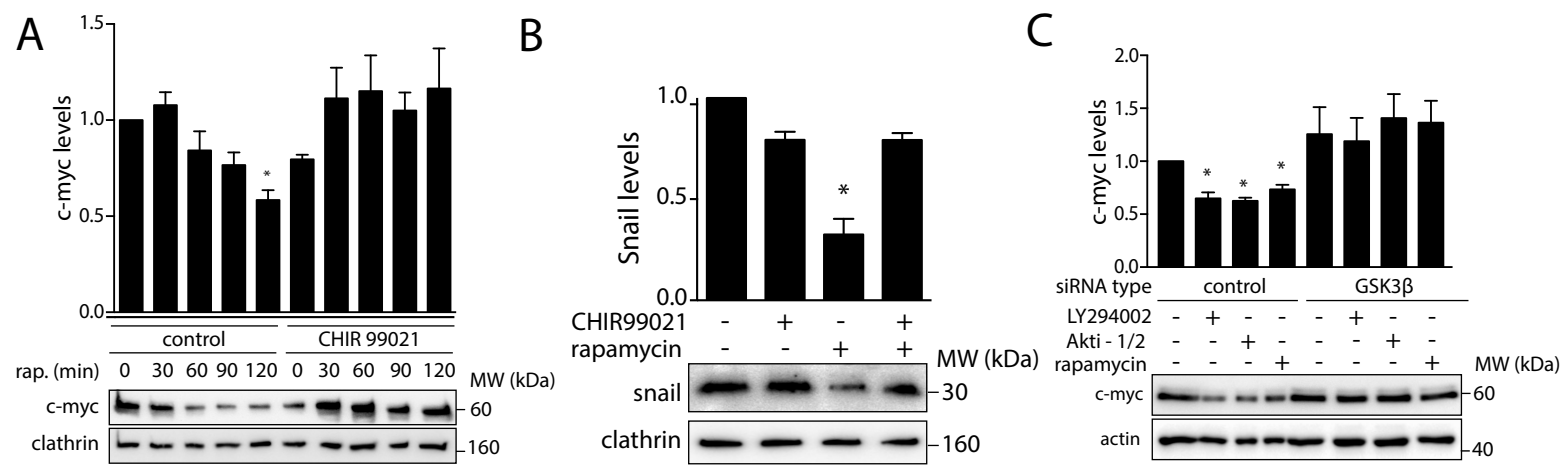

Figure 4. 1 mTORC1 inhibition decreases c-Myc and Snail levels in a GSK3ß-dependent manner. (A\&B), RPE cells were treated with $1 \mu \mathrm{M}$ rapamycin, in the presence or absence of 10 $\mu \mathrm{M}$ CHIR 99021 for the indicated times (A) or $1 \mathrm{~h}(\mathrm{~B})$. Shown are representative immunoblots of whole-cell lysates probed with anti-c-Myc (A), anti-Snail (B), or anti-clathrin heavy chain (load) antibodies. Also shown are mean c-Myc levels \pm S.E. (error bars) $\left(\mathrm{n}<6 ;{ }^{*}, \mathrm{p}<0.05\right)(\mathrm{A})$ or mean Snail levels $(\mathrm{n}=3 ; *, \mathrm{p}<0.05)(\mathrm{B})$ relative to that in the control conditions (absence of CHIR99021 and rapamycin). (C) RPE cells were transfected with siRNA targeting GSK3 $\beta$ or nontargeting siRNA (control) and then treated with either $10 \mu \mathrm{M} \mathrm{LY} 294002,5 \mu \mathrm{M}$, Akti-1/2, or 1 $\mu \mathrm{M}$ rapamycin for $1 \mathrm{~h}$. Shown are representative immunoblots of whole-cell lysates probed with anti-c Myc or anti-actin (load) antibodies as well as mean c-Myc levels $(n=4) . *, p<0.05$ relative to that in the control conditions (absence of LY294002, Akti1/2, and/or rapamycin). All Western blotting quantifications shown have been normalized to loading controls (clathrin or actin). 


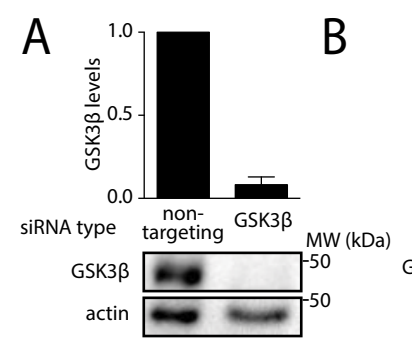

D MDA-MB-231 cells

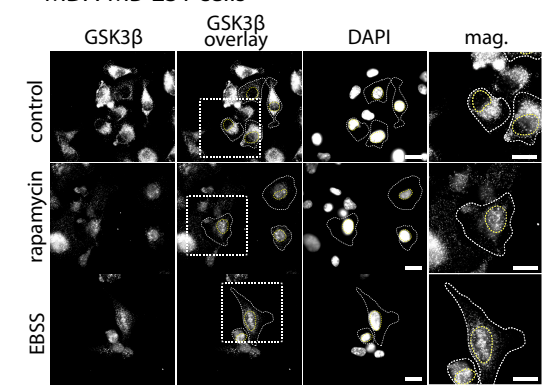

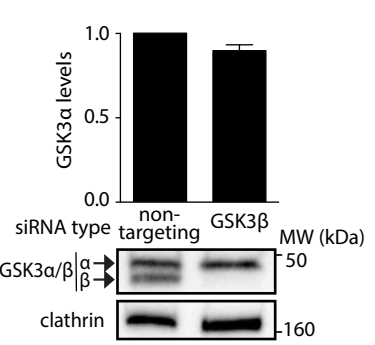
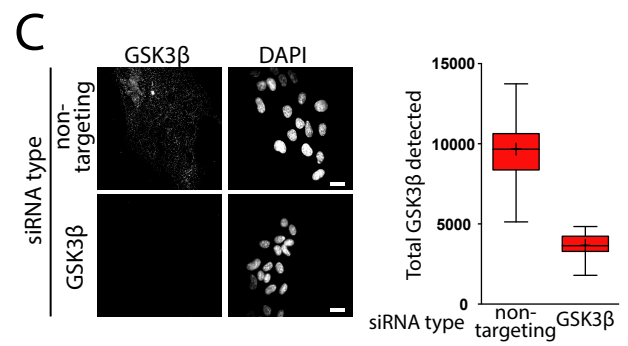

$E$

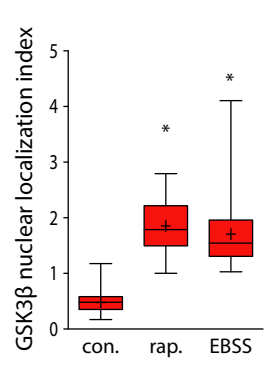

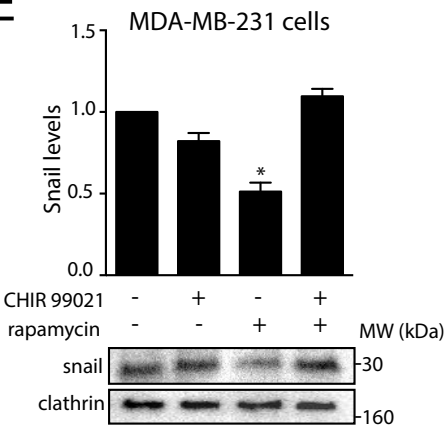

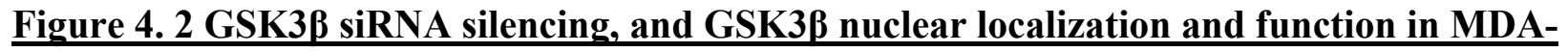
MB-231 cells. (A-C) RPE cells were transfected with siRNA targeting GSK3 $\beta$ or non-targeting siRNA (control), and then subjected to immunoblotting with antibodies that detect GSK3 $\beta$ expression (A) or GSK3 $\alpha$ and GSK3 $\beta$ expression (distinguishable by molecular weight, B), or immunostaining to detect endogenous GSK3 $\beta$, followed by imaging by widefield epifluorescence microscopy (C). These results indicate that siRNA gene silencing of GSK3 $\beta$ was very potent and specific (with no effect on GSK3 $\alpha$ expression). Further, the detection of endogenous GSK3 $\beta$ by immunofluorescence staining is highly specific. (D-E) MDA-MB-231 cells were treated with $1 \mu \mathrm{M}$ Rapamycin for $1 \mathrm{~h}$ or incubated in amino-acid free EBSS media for $2 \mathrm{~h}$. Shown in (D, left panel) are micrographs obtained following staining to detect endogenous GSK $3 \beta$ by widefield epifluorescence microscopy representative of 3 independent experiments, scale $=20 \mu \mathrm{m}$. Also shown in $(\mathrm{D}$, right panel) is the mean GSK3 $\beta$ nuclear localization index \pm $\mathrm{SE}\left(\mathrm{n}=3\right.$ independent experiments, $>30$ cells per condition per experiment); ${ }^{*}, \mathrm{p}<0.05$ relative to control conditions (no inhibitor treatment). Shown in (E) are representative immunoblots of whole-cell lysates probed with anti-Snail (B) or anti-clathrin heavy chain (load) antibodies. Also shown are mean Snail levels $\pm \mathrm{SE}, \mathrm{n}=3 ;^{*}, \mathrm{p}<0.05$, relative to that in the control conditions (no inhibitor treatment) 


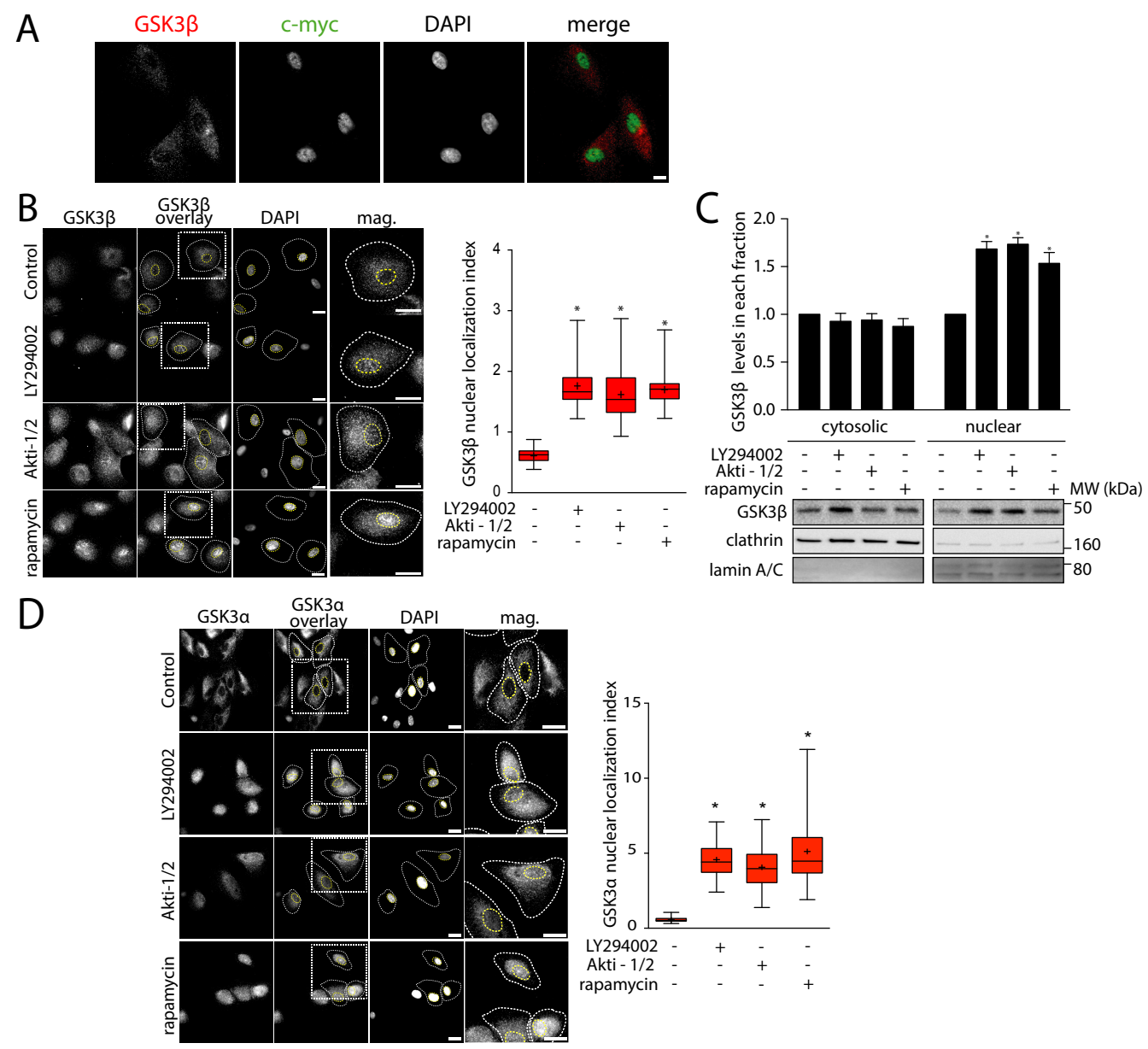

Figure 4. 3 Inhibition of PI3K/Akt/mTORC1 signals promotes GSK3ß nuclear localization. (A) representative images obtained by wide-field epifluorescence microscopy of control RPE cells (no inhibitor treatment) stained to detect endogenous GSK3 $\beta$ or c-Myc, with DAPI stain to identify the nucleus. Scale bar, $20 \mu \mathrm{m}$. (B-D), RPE cells were treated with either $10 \mu \mathrm{M}$ LY294002, $5 \mu \mathrm{M}$ Akti-1/2, or $1 \mu \mathrm{M}$ rapamycin for $1 \mathrm{~h}$. Following this treatment, cells were then fixed and stained to detect endogenous GSK3 $\beta$ (B) or GSK3 $\alpha$ (D). Shown for each (left panels) are micrographs obtained by wide-field epifluorescence microscopy representative of three independent experiments. Scale bar, $20 \mu \mathrm{m}$. Also shown for each condition as GSK3 $\beta$ (or $\alpha$ ) overlay are sample cellular and nuclear outlines, and a box corresponding to a magnified image of a single cell. Also shown (right panel) is the mean GSK3 $\beta$ or GSK3 $\alpha$ nuclear localization index \pm S.E. (error bars) $(\mathrm{n}=3,>30$ cells/condition/experiment); $*, \mathrm{p}<0.05$ relative to control conditions (absence of LY294002, Akti1/2, and rapamycin). Shown in C are Western blots of cytosolic and nuclear fractions and mean nuclear GSK3 $\beta$ values \pm S.E. $(n=3) .{ }^{*}, p<0.05$. 

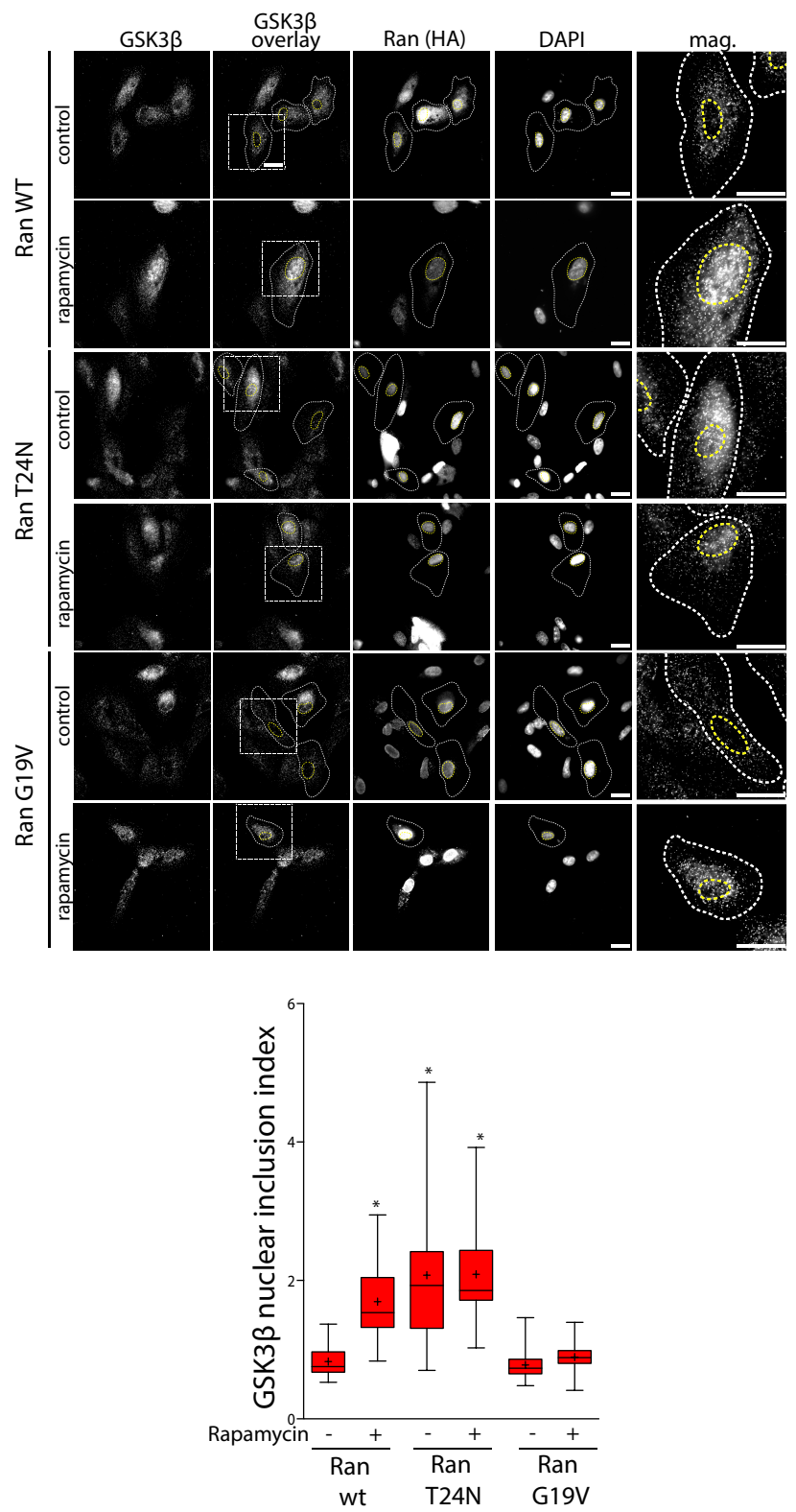

Figure 4. 4 Rapamycin-induced GSK3ß nuclear localization is Ran-dependent. RPE cells were transfected with plasmids encoding HA-tagged WT, T24N, or G19V Ran and then treated with $1 \mu \mathrm{M}$ rapamycin for $1 \mathrm{~h}$, followed by detection of endogenous GSK3 $\beta$ and exogenous HAtagged Ran proteins. Shown (top) are micrographs obtained by wide-field epifluorescence microscopy representative of three independent experiments. Scale bar, $20 \mu \mathrm{m}$. Also shown for each condition as GSK3 $\beta$ overlay are sample cellular and nuclear outlines and a box corresponding to a magnified image of a single cell. Also shown (bottom) is the mean GSK3 $\beta$ nuclear localization index \pm S.E. (error bars) $\left(\mathrm{n}=3,>30\right.$ cells/condition/experiment); ${ }^{*}, \mathrm{p}<0.05$ relative to control conditions (no rapamycin treatment). 


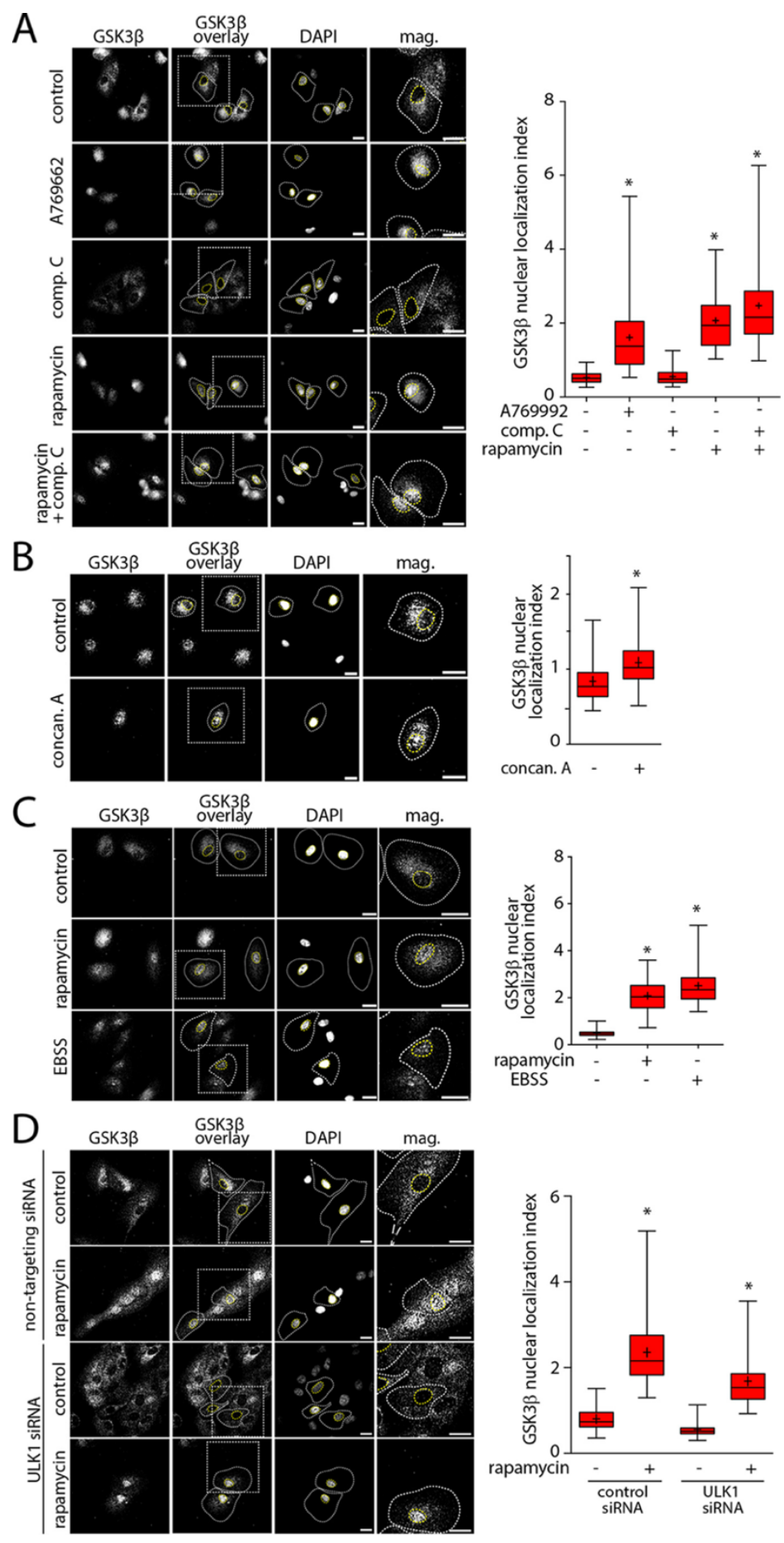

Figure 4. 5 mTORC1 integrates multiple signals to control GSK3ß nuclear localization. RPE cells were treated with either $100 \mu \mathrm{M}$ A769662, $5 \mu \mathrm{M}$ compound $\mathrm{C}$, or $1 \mu \mathrm{M}$ rapamycin, alone or in combination for $1 \mathrm{~h}(\mathrm{~A})$; with $1 \mu \mathrm{M}$ concanamycin for $1 \mathrm{~h}(\mathrm{~B})$; or with amino acid-free EBSS medium for $2 \mathrm{~h}(\mathrm{C})$. D, RPE cells were transfected with siRNA targeting ULK1 or nontargeting siRNA (control). Following knockdown, RPE cells were treated with $1 \mu \mathrm{M}$ rapamycin for $1 \mathrm{~h}$ and then were fixed and stained to detect endogenous GSK3 $\beta$. Shown for each of these (left) are micrographs obtained by wide-field epifluorescence microscopy representative of three independent experiments. Scale bar, $20 \mu \mathrm{m}$. Also shown for each condition as GSK3 $\beta$ overlay are sample cellular and nuclear outlines and a box corresponding to a magnified image of a single cell. Also shown (right) is the mean GSK $3 \beta$ nuclear localization index \pm S.E. (error bars) $(n=3$ independent experiments, $>30$ cells/condition/experiment). ${ }^{*}, \mathrm{p}<0.05$ relative to the non inhibitor-treated condition (and in the control siRNA sample for D) of each experiment. 

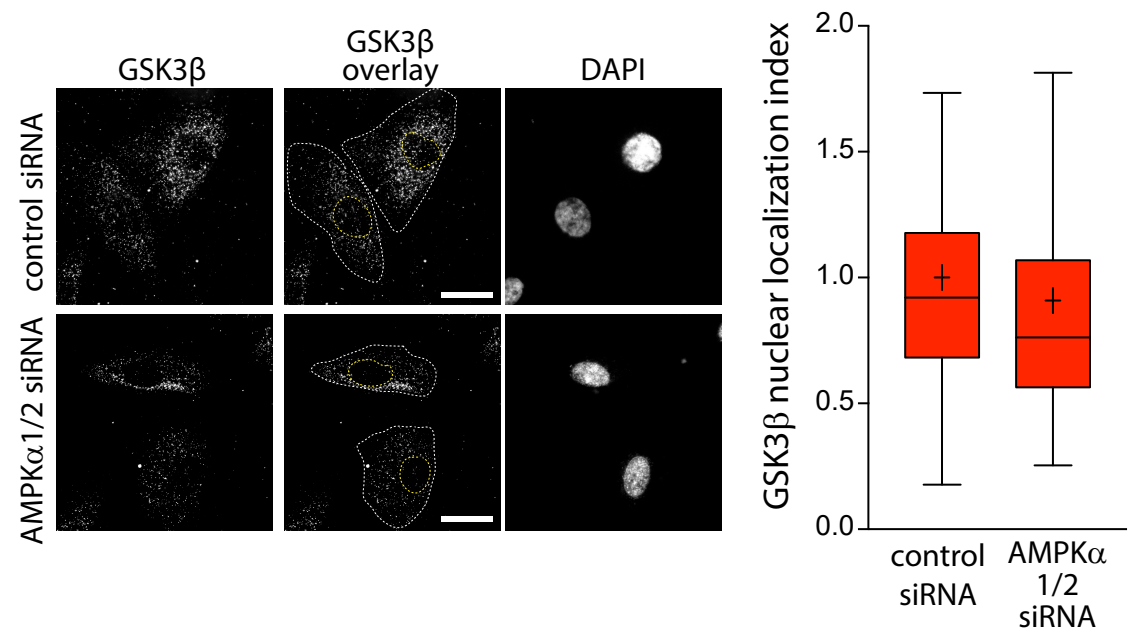

Figure 4. 6 AMPK silencing does not impact GSK3ß nuclear localization. RPE cells were transfected with siRNA targeting both isoforms of the $\alpha$ catalytic subunit of AMPK $(\alpha 1 / \alpha 2)$ or nontargeting siRNA (control), as previous described in (Ross et al., 2015), then fixed and stained to detect endogenous GSK3 $\beta$. Shown (left panel) are micrographs obtained by widefield epifluorescence microscopy representative of 3 independent experiments, scale $=20 \mu \mathrm{m}$. Also shown for each condition as 'GSK3 $\beta$ overlay' are sample cellular and nuclear outlines. Also shown (right panel) is the mean GSK $3 \beta$ nuclear localization index $\pm \mathrm{SE}(\mathrm{n}=3$ independent experiments, $>30$ cells per condition per experiment). 

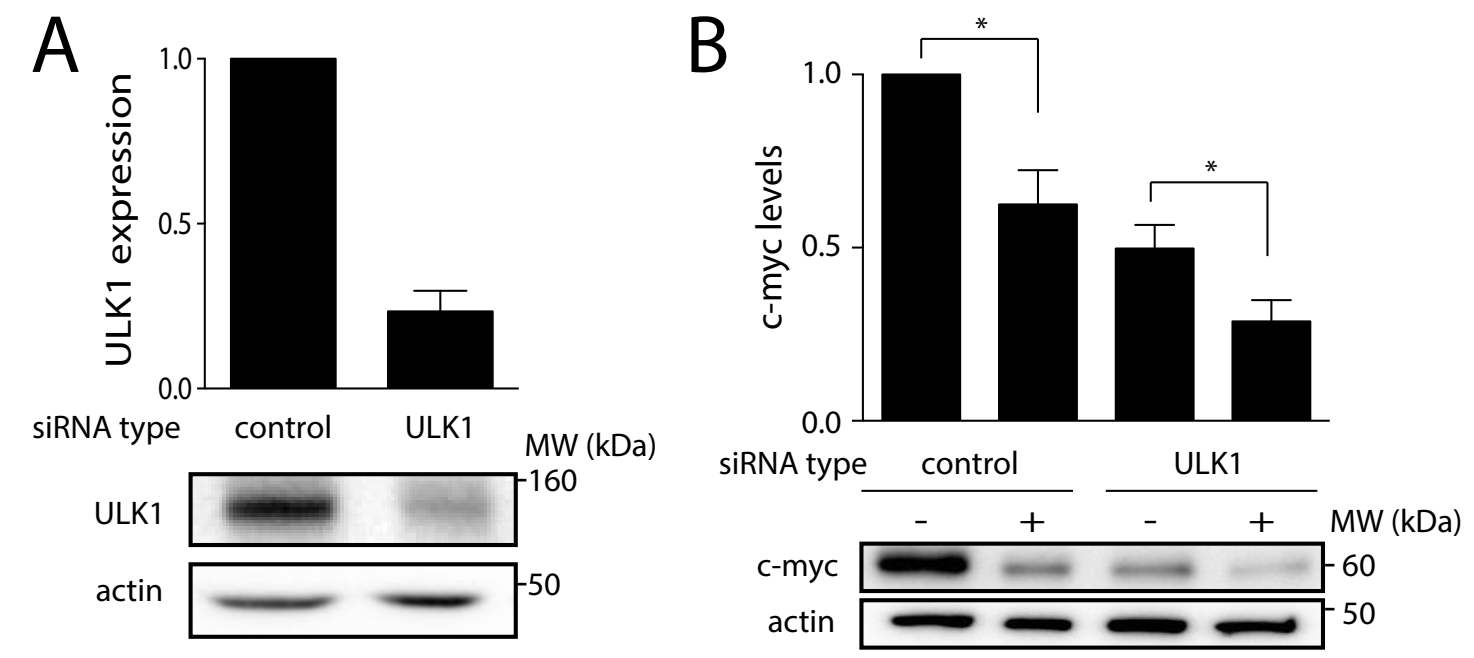

Figure 4. 7 Inhibition of autophagy induction by ULK1 siRNA gene silencing does not impact rapamycin-induced GSK3ß nuclear localization or reduction in c-Myc levels. RPE cells were transfected with siRNA targeting ULK1 or non-targeting siRNA (control). (A) Following knockdown, whole cell lysates were subjected to immunoblotting with antibodies specific for ULK1 or actin (load). Shown are representative immunoblots and mean ULK1 protein expression. (B) Following knockdown, RPE cells were treated with $1 \mu \mathrm{M}$ rapamycin for $1 \mathrm{~h}$. Shown are representative immunoblots of whole cell lysates probed with anti-c-Myc or anti clathrin heavy chain (load) antibodies. Also shown are mean c-Myc levels $\pm \mathrm{SE}, \mathrm{n}=3$, $*, p<0.05$. While ULK1 silencing on its own impacts c-Myc expression, ULK1 silencing does not impact the reduction in c-Myc levels induced by rapamycin treatment. 


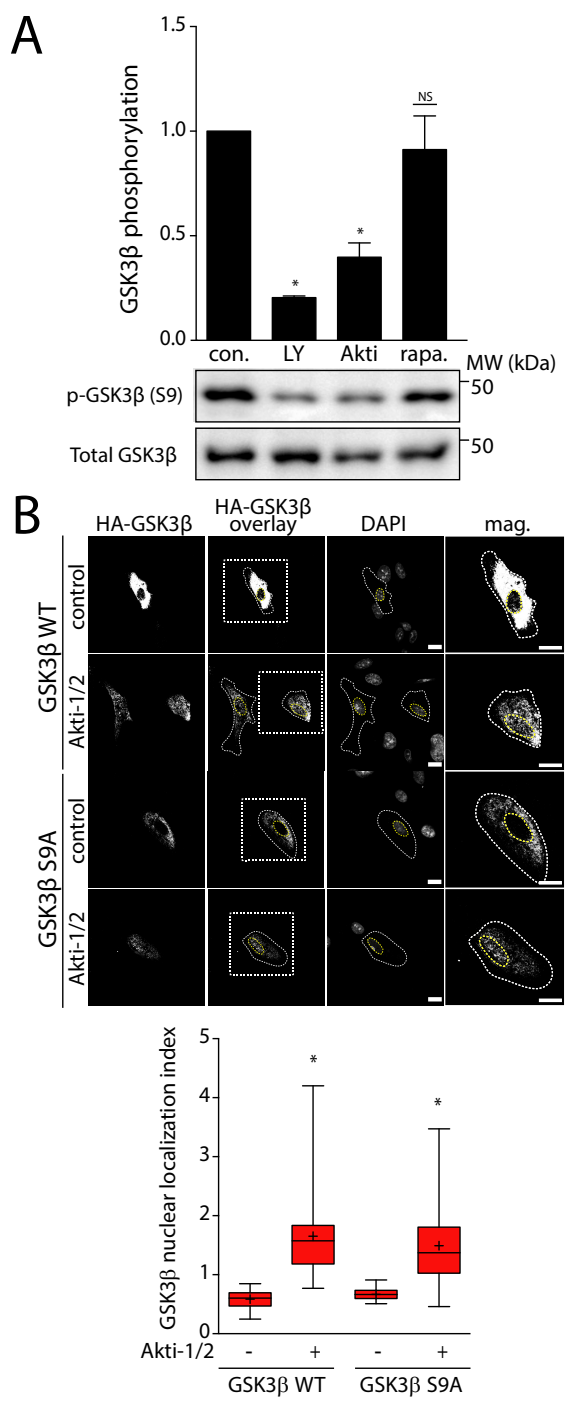

Figure 4. 8 GSK3ß Ser-9 phosphorylation is not required for GSK3ß nuclear localization induced by inhibition of PI3K-Akt-mTORC1 signals. (A) RPE cells were treated with either 10 $\mu \mathrm{M} L Y 294002,5 \mu \mathrm{M}$ Akti-1/2, or $1 \mu \mathrm{M}$ rapamycin for $1 \mathrm{~h}$. Shown are representative immunoblots of whole-cell lysates probed with anti-pSer-9 GSK3 $\beta$ or anti-total GSK3 $\beta$ antibodies. Also shown are mean anti-pSer-9 GSK3 $\beta$ levels (normalized to total GSK3 $\beta) \pm$ S.E. (error bars) $(n=3) ; *, p<0.05$ relative to that in the control conditions (absence of LY294002, Akti1/2, and rapamycin). (B) RPE cells were transfected with plasmids encoding HA-tagged WT or S9A GSK3 $\beta$ and then treated with 5 $\mu \mathrm{M}$ Akti-1/2 for $1 \mathrm{~h}$, followed by detection of exogenous HA-GSK3 $\beta$ proteins. Shown (top) are micrographs obtained by wide-field epifluorescence microscopy representative of three independent experiments. Scale bar, $20 \mu \mathrm{m}$. Also shown for each condition as HA-GSK3 $\beta$ overlay are sample cellular and nuclear outlines and a box corresponding to a magnified image of a single cell. Also shown (bottom) is the mean HA-GSK3 $\beta$ nuclear localization index \pm S.E. $(n=3,>30$ cells/condition/experiment); ${ }^{*}, \mathrm{p}<0.05$ relative to control conditions (absence of Akti1/2 treatment). All Western blotting quantifications shown have been normalized to loading controls. 


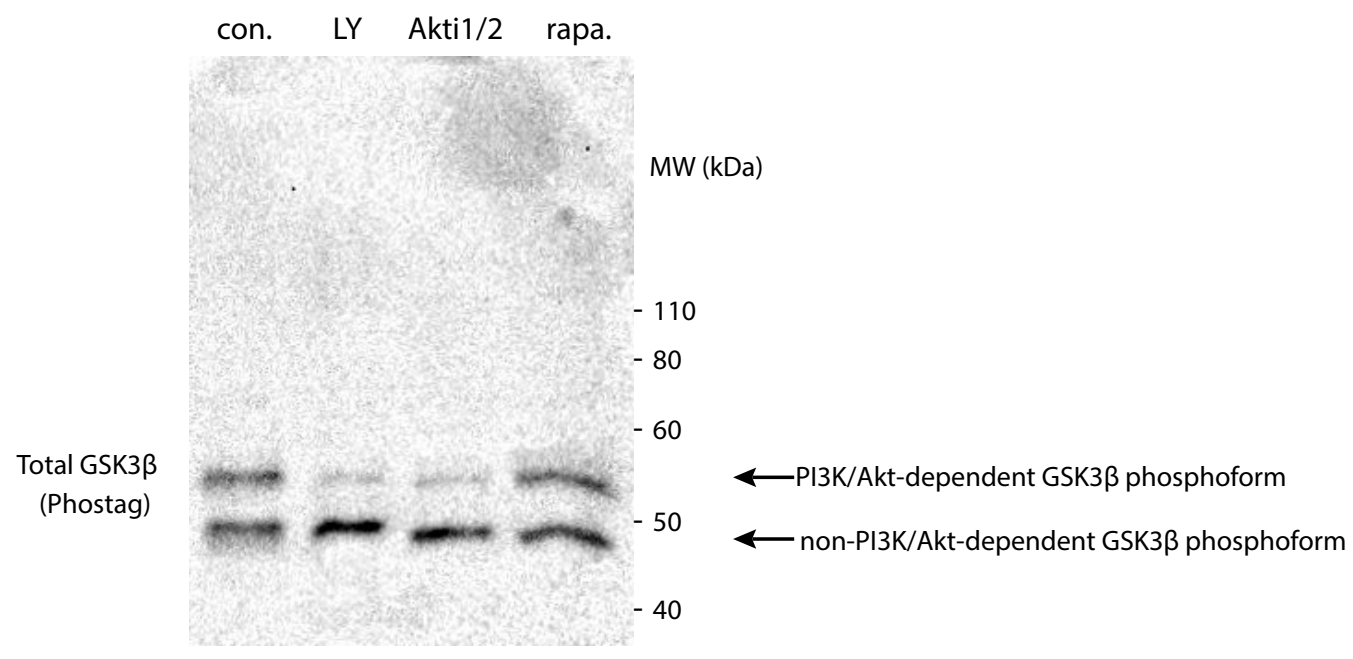

Figure 4. 9 Detection of GSK3ß phosphorylation. RPE cells were treated with either $10 \mu \mathrm{M}$ LY294002, $5 \mu \mathrm{M}$ Akti-1/2, or $1 \mu \mathrm{M}$ rapamycin for $1 \mathrm{~h}$, then subjected to western blotting using anti-GSK3 $\beta$ antibodies, following resolution of distinct phosphorylated species (phosphoforms) by phos-tag SDS-PAGE. Discernable are the pS9 and non-pS9 phosphoforms of GSK3 $\beta$, by the higher apparent molecular weight of the latter, which is reduced upon treatment with LY294002 or Atki-1/2. Rapamycin treatment does not result in any changes in GSK3 $\beta$ phosphorylation discernable by this method. 
Chapter 5: Lysosomes as signalling platforms for control of GSK3 nuclear translocation 


\section{Background and Rationale}

GSK3 $\beta$ is localized to many membrane compartments within the cell including, plasma membrane, early endosome, mitochondria, and nucleus (Reis et al., 2015; Wu and Pan, 2010; Schenck et al., 2008; Zeng et al., 2008; Hoshi et al., 1996). By localizing to each different compartment, GSK3 $\beta$ has the capacity to interact with a unique pool of compartment-specific substrates regulating different signalling and cellular activity in each locale. For example, GSK3 $\beta$ localizes to the plasma membrane through interactions with Axin, allowing GSK3 $\beta$ to play a major role in regulation of Wnt signalling by control of $\beta$-catenin phosphorylation and degradation (Wu and Pan, 2010; Zeng et al., 2008). GSK3 $\beta$ also localizes to APPL1 early endosomes, which allows GSK3 $\beta$ to regulate Dynamin1-dependent endocytosis (Reis et al., 2015; Schenck et al., 2008).

In addition to the more established membrane compartments to which GSK3 $\beta$ exhibits localization, there is evidence of GSK3$\beta$ regulating components of the lysosome, which suggests that GSK3 $\beta$ may also localize to the lysosome, either in part or under specific contexts. Raptor is an mTORC1 component that is phosphorylated by GSK3 $\beta$, thus enhancing mTORC1 activity (Stretton et al., 2015). In addition and showing the complexity of signalling regulation of mTORC1, GSK3 $\beta$ also negatively regulates mTORC1 by activating TSC 2 through phosphorylation of S1337 and S1341 (Ka et al., 2014; Inoki et al., 2006), and both mTORC1 and TSC2 have been reported to localize to the lysosome (Liu et al., 2016; Azoulay-Alfaguter et al., 2015). By regulating different components of the lysosome, GSK3 $\beta$ may also potentially localized to the lysosome, but whether this is the case and the mechanism of GSK3 $\beta$ lysosomal 
localization has yet to be elucidated. In this chapter, I examine the localization of GSK3 $\beta$ to the lysosome as well as to several other membrane compartments (endosomes) to which GSK3 $\beta$ is known to localized, to determine if GSK3 $\beta$ can concomitantly exhibit partial localization to several distinct endosomal compartments.

Membrane traffic is an important component of cellular physiology, as it is required for signalling, organelle and cargo organization, and aspects of metabolism. Rab7 is a GTPase responsible for Late Endosome-Lysosome fusion (Poteryaevet al., 2010), an important event for lysosome organization and signalling. Perturbations in Rab7 GEF hVps39 (important component for Rab 7 activation), prevented early endosome to late endosome conversion and also inhibited mTORC1/S6K1 activation (Flinn et al., 2010). This suggests that Rab7, and thus membrane traffic flow from the early endosome to the lysosome is an important regulator of mTORC1 signalling. This in turn also suggests that GSK3 $\beta$ may be regulated by membrane traffic and Rab7, which I also examine here.

mTORC1 indirectly senses amino acids through regulation of the GATOR complex by the levels of specific amino acids in the cytoplasm, notably leucine and arginine. The GATOR complex is a lysosome tether protein that senses amino acid availability (Shen et al., 2019; Wolfson et al., 2017; Bar-Peled et al., 2013). Under amino abundant conditions GATOR complex is inhibited allowing for Rag proteins to activate mTORC1 (Shen et al., 2019; Wolfson et al., 2017; Bar-Peled et al., 2013). The major component of GATOR1 is DEPDC5 and BioID data from my collaborators (In collaboration Hesketh, G and Gingras, AC), show strong interaction between GSK3 $\alpha / \beta$ to DEPDC5. In this chapter, I examine the importance of 
DEPDC5 on GSK3 $\beta$ membrane localization and mTORC1 dependent nucleocytoplasmic shuttling.

Currently it is not well understood how membrane traffic and the lysosome may serve to organize signalling by mTORC1 to control GSK3 $\beta$ localization and its activity towards downstream targets. Furthermore, is it also not well understood how metabolic sensing through the GATOR complex, or perhaps even direct binding of GSK3 $\beta$ to DEPDC 5 , may control GSK3 $\beta$ localization.

To understand how membrane traffic and the lysosome control GSK3 $\beta$ localization and function. I will first characterize the localization and distribution of GSK3 $\beta$ to different specific endocytic membrane compartments. Next, I will characterize how membrane traffic and the lysosome contributes to GSK3 $\beta$ nucleocytoplasmic shuttle and its activity towards nuclear transcription factor, c-Myc, by studying the effect of Rab7 perturbation on these parameters. Finally, I will look to understand how GATOR complex components and its metabolic sensing ability contribute to GSK3 $\beta$ localization to different membrane components within the cell.

My working hypothesis for this chapter was that the organization signalling from mTORC1 to GSK3 $\beta$ requires the lysosome is an important signalling hub, since the lysosomes contain important metabolic sensory components that regulates GSK3 $\beta$ membrane localization and nucleocytoplasmic shuttling. The specific objectives for this chapter included: 
1) Determine if GSK3 $\beta$ exhibits localization to the lysosome in addition to localization to other endocytic membrane compartments

2) Determine how membrane traffic impacts GSK3 $\beta$ localization

3) Determine how membrane traffic impacts GSK3 $\beta$ function

4) Determine how lysosomal protein GATOR1 contributes to GSK3 $\beta$ membrane localization and mTORC1 dependent nucleocytoplasmic shuttling of GSK3 $\beta$

\section{$5.1 \mathrm{GSK} 3 \beta$ is localized to several distinct membrane compartments within the cytoplasm,} including the lysosome

Active mTORC1 is recruited to the surface of the lysosome (Sancak et al., 2008). Together with our observations that mTORC1 controls GSK3 $\beta$ nuclear localization, this suggests that (i) mTORC1 control of GSK3 $\beta$ may occur at lysosomes and (ii) control of GSK3 $\beta$ nuclear localization by mTORC1 may require lysosomal membrane traffic. To determine if a pool of GSK3 $\beta$ is indeed localized to lysosomes concomitantly to GSK3 $\beta$ recruitment to other endomembrane compartments, I systematically examined the localization of endogenous GSK3 $\beta$ relative to APPL1 and EEA1 early endosomes, and to lysosomes demarked by LAMP1. I observed punctate distribution of endogenous GSK3 $\beta$ within the cytoplasm, with some visible overlap with each of APPL1, EEA1 and LAMP1 (Fig. 5.1A-C, left panels).

To determine if the overlap observed between GSK3 $\beta$ and each marker was specific, I used quantification by Manders' coefficient to compare overlap between real pairs of image channels, as well as between pairs of images with scrambled channel spatial position (one pair of 
the images undergoes a spatial randomization, where it is rotated at $180^{\circ}$; previously described by Bone et al., 2016). This revealed specific GSK3 $\beta$ recruitment to each membrane compartment, since there was clearly statistically significantly different localization detected by Manders' coefficient between GSK3 $\beta$ and each endosomal compartment in real image pairs compared to the "scrambled" image pairs (Fig. 5.1A-C). Limitation with Manders' analysis is the possibility of acquired images containing background signal, nonspecific antibody labelling, or autofluorescence, thus another quantification that also measures colocalization that I used was Pearson's coefficient (Dunn et al., 2011). For real paired images and scrambled images, Pearson's coefficient obtained similar results to the Manders' coefficient (Fig. 5.2A), thus concluding that there is specific localization to the lysosome (or endosome) and that there is a significant difference in the Pearson's value in real images and the scrambled images. This indicates that GSK3 $\beta$ indeed exhibits mTORC1 regulates GSK3 $\beta$ partial yet specific localization to several distinct endomembrane compartments, including APPL1 and EEA1 early endosomes, and late endosomes/lysosomes demarked by LAMP1.

GSK3 $\beta$ has been described as undergoing recruitment into MVBs and degradation in lysosomes in response to Wnt signalling (Taelman et al., 2010). To further examine how GSK3 $\beta$ may localize to lysosomes, I employed structured illumination microscopy (SIM). Using this method, I was able to resolve the limiting membrane of lysosomes demarked by LAMP1 fluorescence staining (Fig. 5.1D). Importantly and consistent with the images that were obtained by spinning disc confocal microscopy (Fig. 5.1C), using SIM, GSK3 $\beta$ fluorescence staining was readily observed in punctate structures, some of which were associated with lysosomes. Using automated segmentation of the lysosomes in SIM images, GSK3 $\beta$ puncta were preferentially 
detected on the limiting membrane versus the interior of lysosomes (SIM images were obtained in collaboration with Yip C, Kim P, and Vissa, A (U of T and SickKids) (Fig. 5.1D). These results indicate that a subset of GSK3 $\beta$ in the cytoplasm exhibits association with the lysosome, either restricted to sub-domains of the lysosomal surface (Kaushik et al., 2006) or in structures associated with the lysosome, such as within membrane contact sites (Chu et al., 2015. Furthermore, lysosomal recruitment of GSK3 $\beta$ is not in the lumen of the lysosome, making it unlikely that much of lysosome-associated GSK3 $\beta$ is undergoing degradation under these conditions.

5.2 Control of GSK3 3 nuclear localization and c-Myc levels requires Rab7-mediated lysosomal $\underline{\text { membrane traffic }}$

Given the localization mTORC1 (Zoncu et al., 2009) and partial localization of GSK3 $\beta$ (Fig. 5.1C-D) to or near the lysosome, I next sought to determine the role of late endosome/lysosome membrane traffic to mTORC1-dependent control of GSK3 $\beta$ nuclear localization. To do so, I made use of expression of dominant-interfering Rab7 mutant that is constitutively GDP-bound (T22N), which disrupts membrane traffic at the late endosome/lysosome (Azoulay-Alfaguter et al., 2011). Cells expressing Rab7 T22N exhibited a significant increase in nuclear GSK3 $\beta$, even in the absence of rapamycin treatment, while cells expressing Rab7 WT exhibited a largely cytoplasmic GSK3 $\beta$, similar to cells not transfected to express Rab7 constructs (Fig. 5.3A). Furthermore, cells expressing Rab7 T22N exhibited a depletion of GSK3 $\beta$ from lysosomes, observed by overlap of endogenous GSK3 $\beta$ and LAMP1 and quantified by Manders' coefficient (Fig. 5.2C). 
The localization of GSK3 $\beta$ to the nucleus upon disruption of Rab7 could result from effects of a broad disruption in endocytic membrane traffic (since GSK3 $\beta$ localizes to many endocytic compartments), or to a specific disruption of the lysosomes. To distinguish these possibilities, I examined the effect of perturbation of APPL1, which prevents formation of APPL1 endosomes. In contrast to the nuclear accumulation of GSK3 $\beta$ in cells expressing Rab7 T22N, silencing of APPL1 (Fig. 5.2B) to disrupt early endosome membrane traffic did not impact GSK3 $\beta$ nuclear localization (Fig. 5.3B). These results indicate that membrane traffic at the late endosome/lysosome may be selectively important to organize mTORC1 signals leading to control of GSK3 $\beta$ nuclear localization.

In order to determine the functional consequence of Rab7-dependent control of GSK3 $\beta$ nuclear localization, I examined the effect of expression of Rab7 T22N on GSK3 $\beta$-dependent cMyc levels. Cells expressing Rab7 T22N exhibited a stark reduction in c-Myc levels relative to cells expressing Rab7 WT (Fig. 5.3C). Importantly, treatment of cells expressing Rab7 T22N with the GSK3 $\beta$ inhibitor CHIR99021 restored c-Myc levels to that observed in cells expressing Rab7 WT (Fig. 5.3C). Taken together, these results indicate that control of GSK3 $\beta$ nuclear localization requires Rab7-dependent late endosome/lysosomal membrane traffic, reflecting perhaps the role of lysosomes as platforms for mTORC1 signalling required to negatively regulate GSK3 $\beta$ nuclear translocation. 


\section{$\underline{5.3 \text { Specific amino acids acutely promote GSK3 nuclear export }}$}

In amino acid starved cells, TFEB translocates into the nucleus (Bajaj et al., 2018), but when amino acids are replenished, TFEB is exported out of nucleus in an mTORC1-dependent manner (Napolitano et al., 2018). This suggests that a similar mechanism by which mTORC1 acutely controls the nucleocytoplasmic shuttling of GSK3 $\beta$, such that signals such as the abundance of specific amino acid and mitogenic signalling that activates PI3K-Akt may acutely trigger GSK3 $\beta$ nuclear exit. Thus, I next looked to explore the mechanism and regulation of nuclear export, by specifically examining the role of mitogenic and metabolic signals. To do so, I starved RPE cells of amino acids and serum for $2 \mathrm{hrs,} \mathrm{promoting} \mathrm{GSK3} \beta$ nuclear translocation (Fig. 5.4). Subsequent to this, I treated these cells with various metabolic and mitogenic signals for 10-30 mins and examined GSK3 $\beta$ localization. RPE cells treated with arginine or leucine exhibited strong GSK3 3 nuclear export to the cytoplasm. In contrast, initially amino-acid starved RPE cells subsequently treated with media containing only non-essential amino acids exhibited GSK3 $\beta$ that remained largely nuclear (Fig. 5.4). In addition, treatment of cells that are initially amino acids starved with only EGF to activate PI3K-Akt signalling also resulted in GSK3 $\beta$ localization that was remained largely nuclear (Fig. 5.4). Interestingly, treating cells that were initially amino acid deprived cells, but subsequently co-treated with both EGF and non-essential amino acids resulted in strong nuclear export of GSK3 $\beta$ (Fig. 5.4). This suggest that metabolic signalling from amino acids and EGF mitogenic signalling are both are important for controlling mTORC1 mediated nuclear export of GSK3 $\beta$. 


\subsection{DEPDC5 controls lysosomal localization of GSK3 $\beta$ and mTORC1-dependent nucleocytoplasmic shuttling of GSK3}

The GATOR1 complex is localized at the lysosome and is the indirect amino acid sensor for mTORC1 (Shen et al., 2018). In the absence of amino acid, mTORC1 is inhibited, in part as a result of the GAP activity of DEPDC5 (main subunit of GATOR1) that promotes GTP hydrolysis of Rag GTPases, thus preventing mTORC1 recruitment to the lysosome (Shen et al., 2018). To identify the significance of GATOR1 complex to GSK3 localization, I initiated a collaboration with the lab of Dr. Anne Claude Gingras (TSRI) to study protein-protein interactions of lysosomal proteins using a technique called BioID (Roux et al., 2018). Using this method to study a number of interactions, the Gingras lab (In collaboration with Hesketh, G and Gingras, AC) identified both GSK $3 \alpha$ and $\beta$ are exhibit proximity to GATOR1 subunit, DEPDC5, reflective of very strong binding of GSK3 with DEPDC5 (Appendix Fig. A3). This result indicates that a pool of GSK $3 \alpha$ and $\beta$ may localize to the lysosome as a result of interaction with and tethering by the lysosomal GATOR1 subunit DEPDC5.

The novel interaction of GSK3 with the amino acid sensor complex GATOR1 and the ability of amino acids sensed (indirectly) by GATOR1 (leucine and arginine) under conditions in which mTORC1 activity remains low (e.g. in the absence of co-stimulation with mitogenic signals) suggests that DEPDC5-GSK3 interaction may regulate 1) lysosomal localization of GSK3 $\beta$ and 2) GSK3 $\beta$ nucleocytoplasmic shuttling directly. To understand how DEPDC5 contributes to lysosome localization of GSK3 $\beta$, I examined the effect of DEPDC5 KO on GSK3 distribution on lysosomal localization. I obtained HEK293 cells in which the gene encoding 
DEPDC5 had been inactivated (DEPDC5 KO cells) by CRISPR/Cas9 genome editing form the Gingras lab (In collaboration with Hesketh, G and Gingras, AC). Immunofluorescence microscopy as in (Fig. 5.1C \& D) to detect LAMP1 and GSK3 $\beta$, followed by Manders' coefficient analysis to detect GSK3 $\beta$ colocalization with LAMP1 revealed that DEPDC5 KO cells showed a significant reduction of GSK3 $\beta$ from lysosomes compared to control knock out cells that were subjected to CRISPR/Cas9 with a guide RNA that does not specifically target any gene sequence (Fig. 5.5A). Taken together with my previous results obtained by confocal and SIM imaging that showed that GSK3 $\beta$ localizes to LAMP1 positive lysosomes (Fig. 5.1 C \& D), these results further solidify GSK3$\beta$ as a resident lysosomal protein. In addition, these results indicate that DEPDC5 may acts a lysosomal tether to recruit populations of GSK3 $\beta$ to the lysosomal membrane.

I sought to examine the functional consequence of perturbation of the amino acid sensing function of GATOR1 for control of GSK3 $\beta$ nuclear relocalization. To do so, I examined the effect of amino acid depletion on mTORC1 dependent GSK3 $\beta$ relocalization, in combination with serum starvation. Consistent with my previous results, HEK293 (control KO) cells subjected to amino acid and serum starvation by incubation in EBSS showed a significant increase in GSK3 $\beta$ nuclear localization compared to basal conditions (Fig. 5.5B). In these cells, a subsequent 15 min leucine stimulation after amino acid and serum deprivation resulted in rapid translocation of GSK3 $\beta$ out of the nucleus (Fig. 5.5B). Importantly, DEPDC5 KO cells starved of amino acids did not exhibit nuclear localization of GSK3 $\beta$, as was observed in control KO HEK293 cells (Fig. 5.5B). This suggests two non- mutually exclusive possibilities: 1) that since DEPDC5 suppresses mTORC1 activation, that DEPDC5 KO causes aberrant activation of 
mTORC1 that constitutively prevents the normal signalling that links loss of amino acids to nuclear export of GSK3 $\beta$ or 2) that loss of the lysosomal anchor/tether DEPDC5 perturbs GSK3 $\beta$ recruitment to the lysosome thus uncoupling amino acid sensing from control of GSK3 $\beta$ localization.

To determine if the first possibility above, that of aberrantly elevated mTORC1 in DEPDC5 KO cells constitutively restricting GSK3 $\beta$ from the nucleus even in the absence of amino acids, may be possible, I examined mTORC1 signalling and activity in DEPDC5 KO cells. DEPDC5 KO cells exhibit a robust and constitutive mTORC1 activation, as seen by the phosphorylation of the mTORC1 substrate pS6K (Appendix Fig. A4) (In collaboration with Hesketh, G and Gingras, AC). To determine if this aberrant mTORC1 activity contributed to abnormal control of nuclear localization of GSK3 $\beta$ in DEPDC5 KO cells, cells treated with $1 \mu \mathrm{M}$ rapamycin. In control KO HEK293 cells, rapamycin elicited a robust nuclear translocation of GSK3 $\beta$ (Fig. 5.5C), consistent with previous results I obtained in RPE and MDA-MB-231 cells (Fig. 4.2B \& 4.1.1D). Importantly, in DEPDC5 KO cells, rapamycin treatment also resulted in strong relocalization of GSK3 $\beta$ into the nucleus (Fig. 5.5C). These results indicate that loss of DEPDC5 indeed leads to aberrant high mTORC1 activity, which prevents control of GSK3 $\beta$ nuclear localization by normal cues such as amino acid abundance.

In summary, GSK3 $\beta$ localizes to different endomembrane compartments including EEA1 early endosomes, APPL1 early endosomes, and LAMP1 positive late endosome/lysosomes. Using SIM, GSK3 $\beta$ localizes to the limiting membrane of LAMP1 positive late endosome/lysosomes. Furthermore, GSK3 $\beta$ nuclear localization and activity is also control by membrane traffic via Rab7. GSK3 $\beta$ nuclear export is controlled by amino acid and growth factor 
replenishment. Finally, GATOR1 component DEPDC5, tethers populations of GSK3 $\beta$ to LAMP1 positive late endosome/lysosomes and controls amino acid dependent nuclear problem of GSK3 $\beta$. 


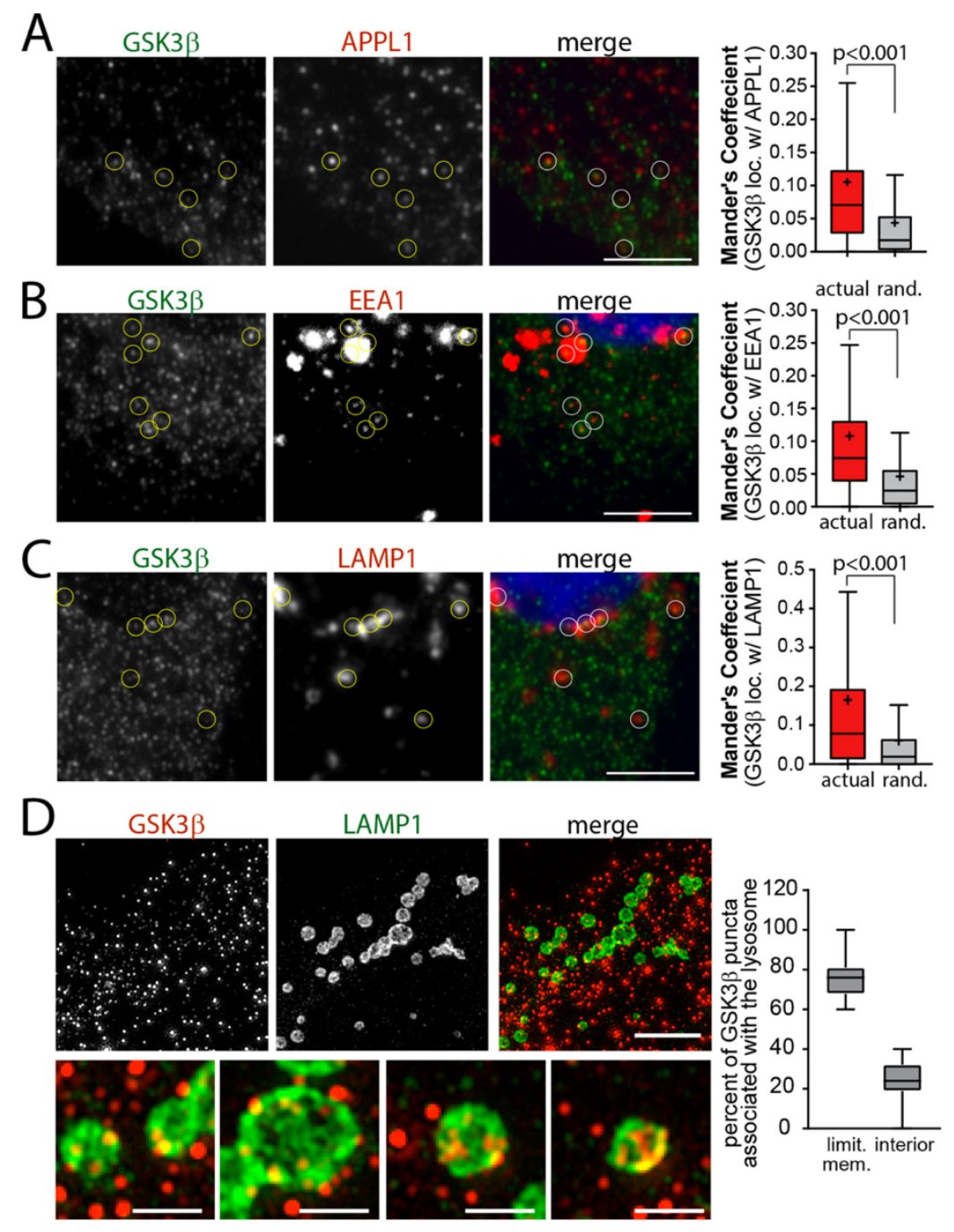

Figure 5. 1 GSK3ß exhibits partial localization to several distinct endomembrane compartments. (A-C), RPE cells were fixed and stained to detect endogenous GSK3 $\beta$, together with either endogenous APPL1 (A), EEA1 (B), or LAMP1 (C). Shown are representative images obtained by spinning-disc confocal microscopy, corresponding to a zsection through the middle of the cell. Scale bar, $20 \mu \mathrm{m}$ (left panels). Also shown (right panels) are the median (bar), interquartile range (boxes) and full range (bars) of Manders' coefficients to measure overlap of GSK3 $\beta$ signals with either APPL1 (A), EEA1 (B), or LAMP1 (C) $(n=3$, $>30$ cells/condition/experiment). For each image set, Manders' coefficients were calculated for actual images (actual), as well as images in which the spatial position of one of the channels had been randomized (rand.), to allow resolution of specific GSK3 $\beta$ localization to various endomembrane compartments from random overlap of signals in a field densely populated with fluorescent objects. (D) RPE cell samples prepared similarly as in C were subjected to SIM. Shown (left) are representative micrographs of (endogenous) GSK3 $\beta$ and LAMP1staining morphology. Scale bar, $5 \mu \mathrm{m}$ (top panels) or $1 \mu \mathrm{m}$ (bottom panel). Also shown (right panels) is the distribution of GSK3 $\beta$ puncta following automated segmentation of the lysosome in SIM images into limiting membrane and interior, shown are the median (bar), interquartile range (boxes), and full range (bars) of GSK3 $\beta$ puncta in each region for each lysosome (SIM images were obtained in collaboration with C. Yip, P. Kim and A. Vissa (U of T and SickKids)). 

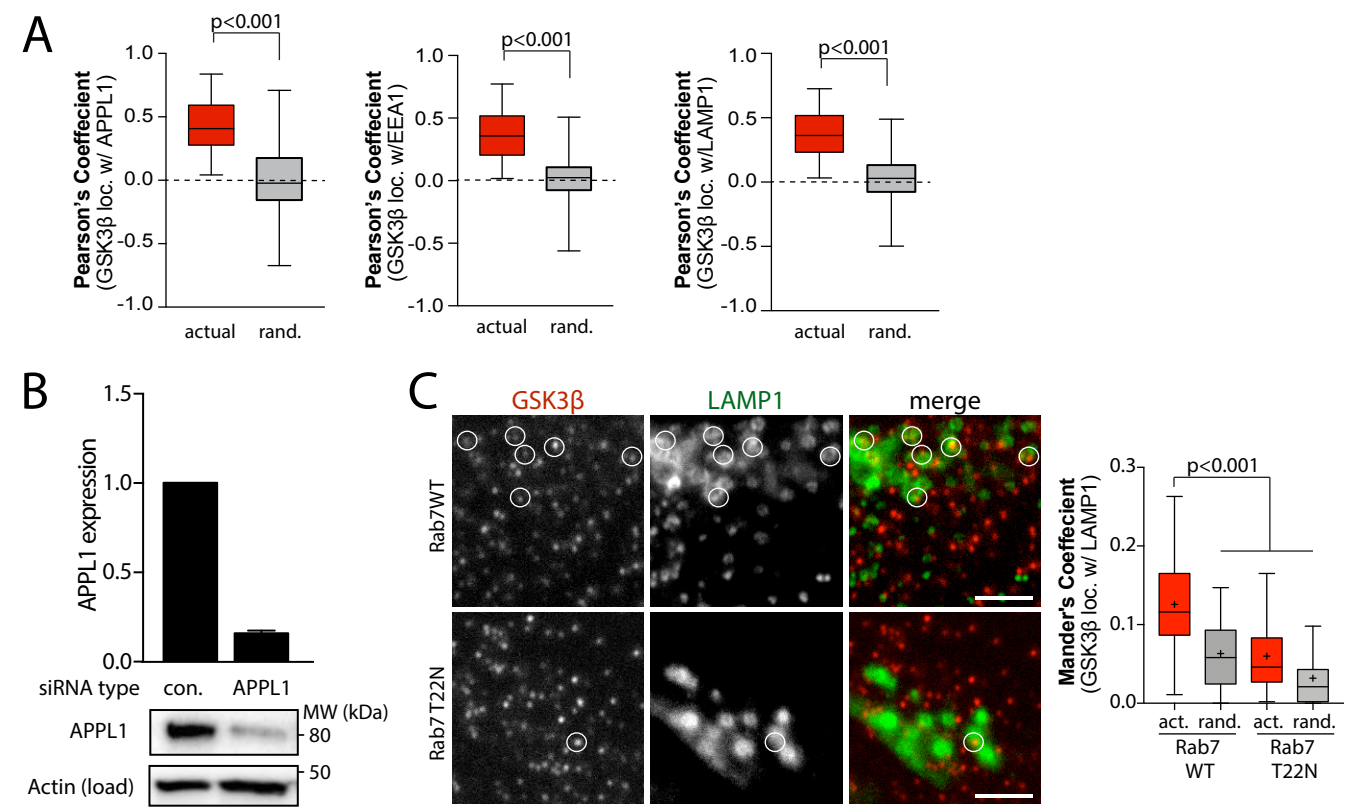

Figure 5. 2 Contribution of Rab7 and APPL1 to GSK3ß localization. (A) The spinning disc confocal images of endogenous GSK3 $\beta$ together with APPL1, EEA1 or LAMP1 shown in Fig. 6A-C were also subjected to co-localization analysis by Pearson's coefficient. Shown are the mean \pm SE of Pearson's coefficient to measure overlap of GSK3 $\beta$ signals with either APPL1 EEA1, or LAMP1, as indicated $(\mathrm{n}=3$ independent experiments, $>30$ cells per condition per experiment). For each image set, Pearson's coefficients were calculated for actual images (labelled 'actual'), as well as images in which the spatial position of one of the channels had been randomized (labelled 'rand.'). These results are consistent with those obtained by Manders' coefficient and indicate that GSK3 $\beta$ exhibits partial but specific localization to several endomembrane compartments, including the late endosome/lysosome demarked by LAMP1. (B) RPE cells were transfected with siRNA targeting APPL1 or non-targeting siRNA (control) as in Fig. 7B. Following knockdown, whole cell lysates were subjected to immunoblotting with antibodies that detect APPL1. Shown are representative immunoblots and the mean $\pm \operatorname{SE}(n=3)$ APPL1 expression (normalized to loading control). (C) RPE cells were transfected with plasmids encoding dsRed-tagged wild-type (WT) or T22N Rab7, then treated with $1 \mu \mathrm{M}$ rapamycin for 1 $\mathrm{h}$ (all as in Fig. 7), followed by detection of endogenous GSK3 $\beta$ and LAMP1. Shown are representative micrographs obtained by widefield epifluorescence microscopy, scale $=5 \mu \mathrm{m}$ (left panels). Also shown (right panels) are the means \pm SE of Manders' coefficients, to measure overlap of GSK $3 \beta$ signals with LAMP1 $(n=3$ independent experiments, $>30$ cells per condition per experiment). For each image set, Manders' coefficients were calculated for actual images (labelled 'actual'), as well as images in which the spatial position of one of the channels had been randomized (labelled 'rand.'). These results show that disruption of Rab7 function results in loss of GSK $3 \beta$ localization to the late endosome/lysosome demarked by LAMP1. 

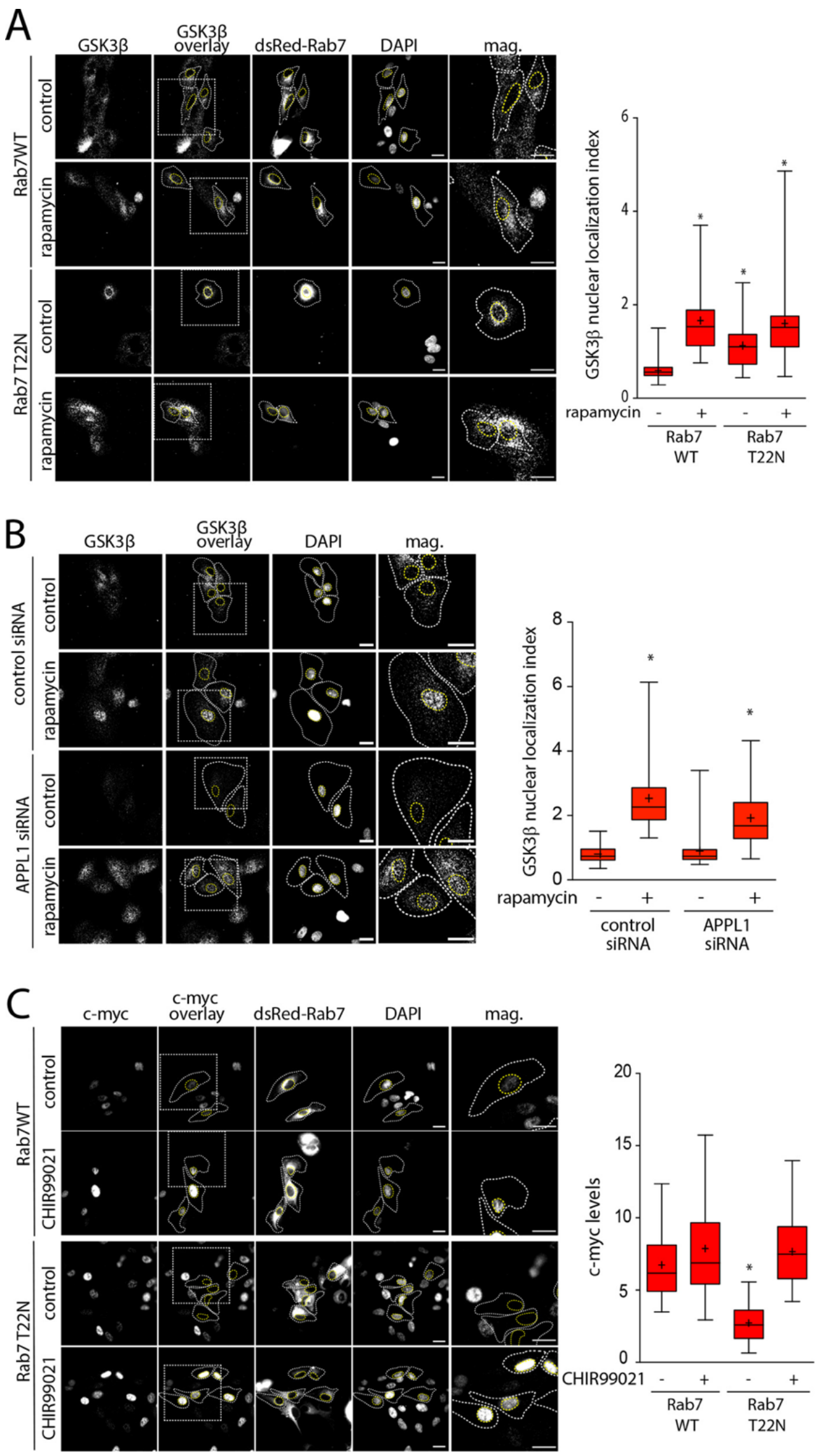


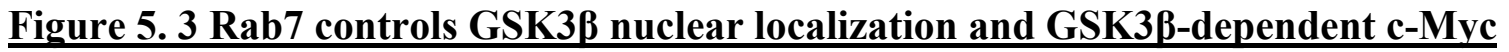
levels. RPE cells were transfected with plasmids encoding dsRed tagged WT or T22N Rab7 (A and C) or transfected with siRNA targeting APPL1 or nontargeting siRNA (control) (B). Some samples were then treated with $1 \mu \mathrm{M}$ rapamycin for $1 \mathrm{~h}$, followed by detection of endogenous GSK3 $\beta$ (A and B) or c-Myc (C). Shown (left panels) are micrographs obtained by wide-field epifluorescence microscopy representative of three independent experiments. Scale bar, $20 \mu \mathrm{m}$. Also shown for each condition as GSK3 $\beta$ overlay (A) or c-Myc overlay (B) are sample cellular and nuclear outlines and a box corresponding to a magnified image of a single cell. Also shown (right panels) is the mean \pm S.E. (error bars) of the GSK3 $\beta$ nuclear localization index $(\mathrm{A}$ and $\mathrm{B})(\mathrm{n}=3,>30$ cells/condition/experiment) or total cellular c-Myc level $(\mathrm{C})\left(\mathrm{n}=3,>30\right.$ cells/condition/ experiment); ${ }^{*}, \mathrm{p}<0.05$ relative to control conditions (no rapamycin or CHIR99021 treatment. 

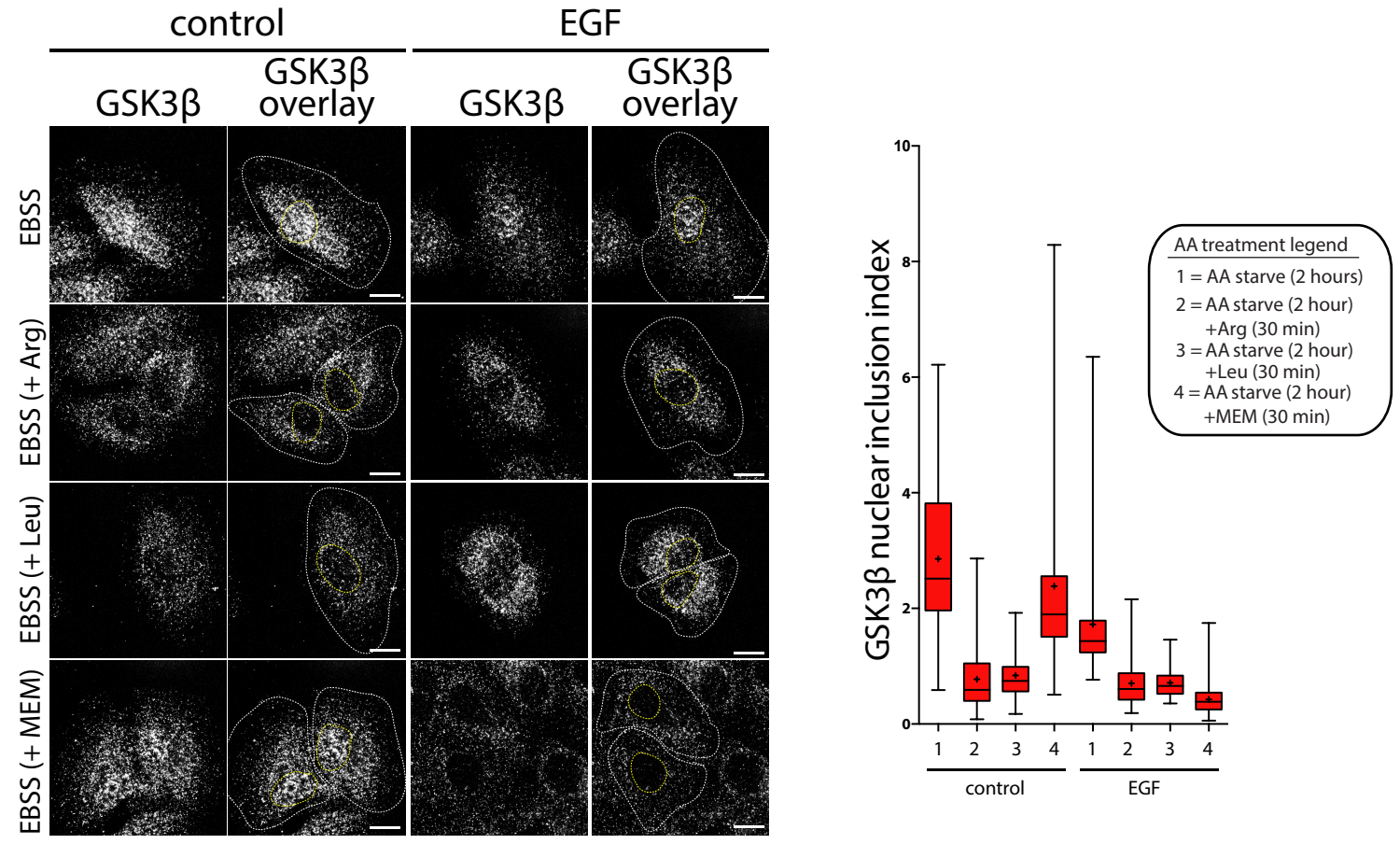

Figure 5. 4 Amino acid starved cells replenished with leucine, arginine, and non-essential amino acids + EGF export GSK3B from the nucleus. RPE cells were starved with EBSS for 2 hrs then treated with either $0.4 \mathrm{mM}$ Arginine, $0.4 \mathrm{mM}$ Leucine, 1:100 MEM non essential amino acids, $+/-5 \mathrm{ng} / \mathrm{ml}$ EGF for 30 minutes. RPE cells were then fixed and stained to detect endogenous GSK3 $\beta$. Shown for each of these (left) are micrographs obtained by wide-field epifluorescence microscopy. Scale bar, $20 \mu \mathrm{m}$. Also shown for each condition as GSK3 $\beta$ overlay are sample cellular and nuclear outlines of single cells. Also shown (right) is the mean GSK3 $\beta$ nuclear localization index \pm S.E. (error bars) $(n=1,>30$ cells/condition). 


\section{A}
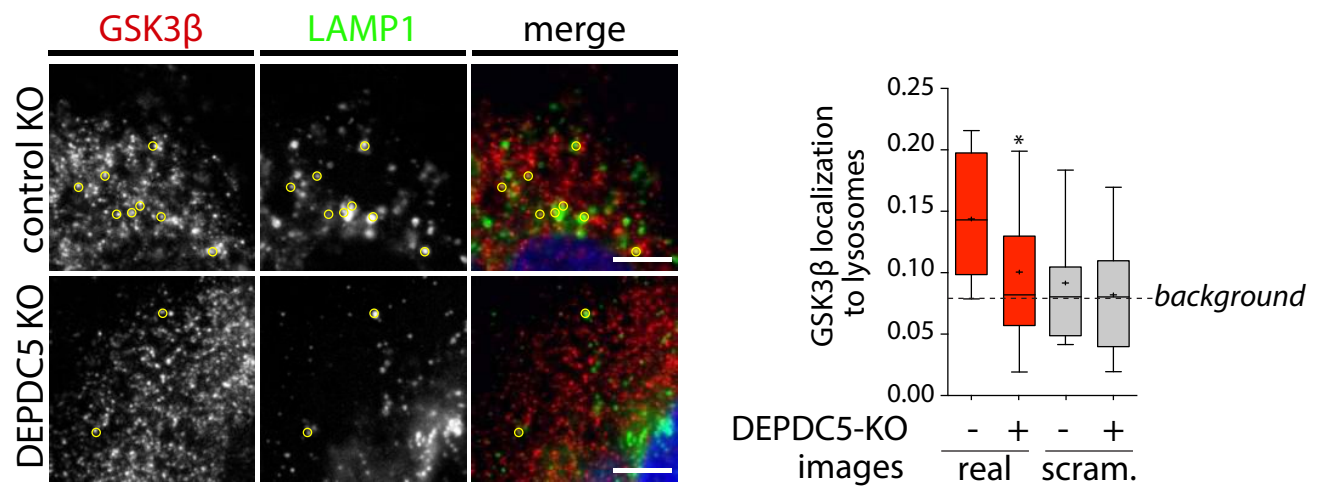

\section{B}
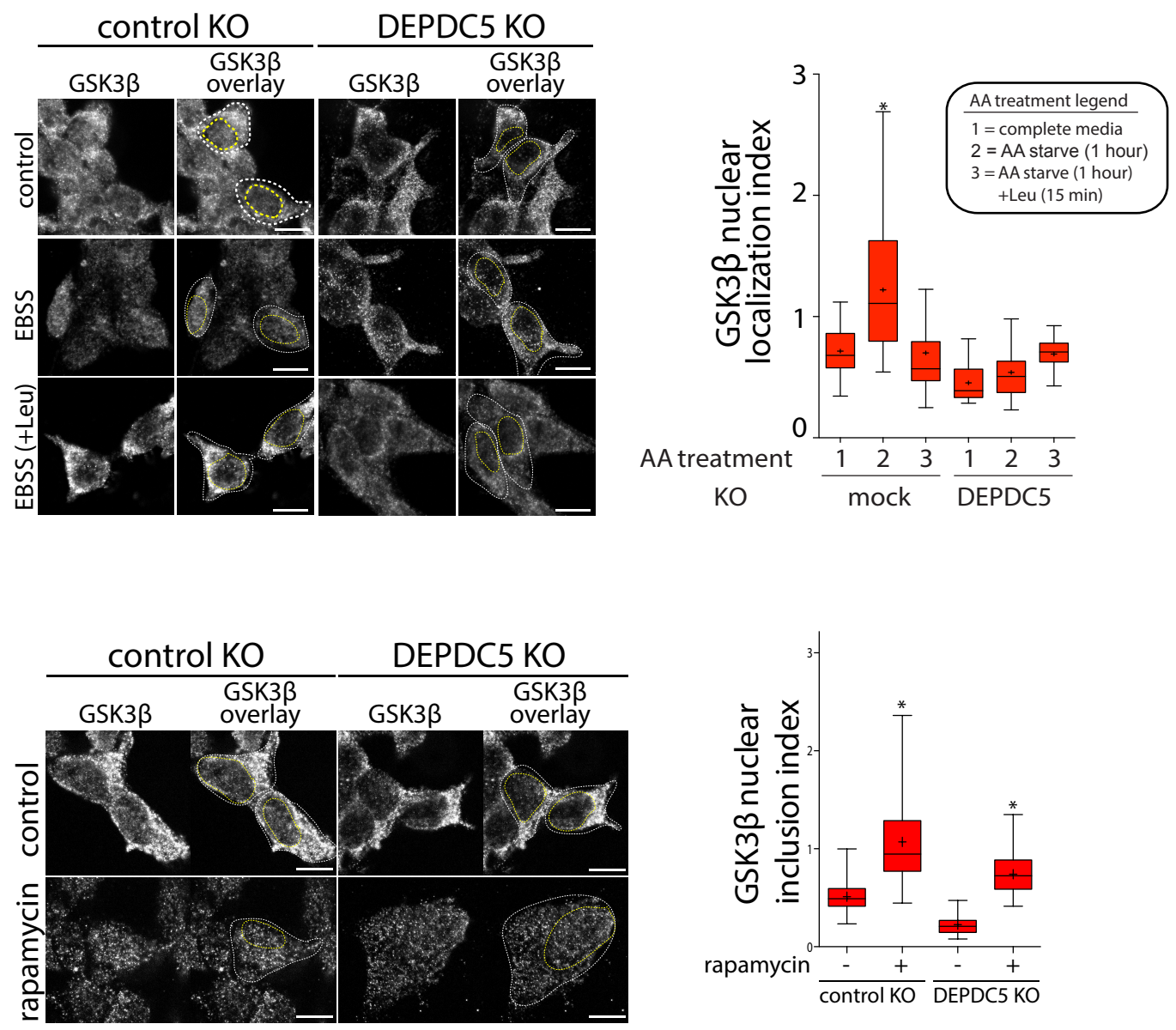


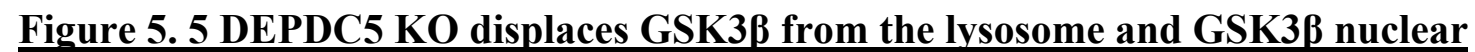

localization program is insensitive to amino acid deprivation. (A-C) HEK293 cells were knocked out of DEPDC5 using CRISPR/Cas9. (A) HEK293 cells were fixed and stained to detect endogenous GSK $3 \beta$ with LAMP1. Shown are representative images obtained by spinning-disc confocal microscopy, corresponding to a z-section through the middle of the cell. Scale bar, $5 \mu \mathrm{m}$. Also shown (right panels) are the median (bar), interquartile range (boxes) and full range (bars) of Manders' coefficients to measure overlap of GSK3 $\beta$ signals with LAMP1 (n $=3,>30$ cells/condition/experiment). For each image set, Manders' coefficients were calculated for actual images (actual), as well as images in which the spatial position of one of the channels had been randomized (rand.), to allow resolution of specific GSK3 $\beta$ localization to various endomembrane compartments from random overlap of signals in a field densely populated with fluorescent objects. (B \& C) representative images obtained by wide-field epifluorescence microscopy of control KO vs DEPDC5 KO HEK 293 cells stained to detect endogenous GSK3 $\beta$. Scale bar, $10 \mu \mathrm{m}$. Control KO vs DEPDC5 KO HEK 293 cells were treated with $1 \mathrm{~h}$ EBSS $+/-0.04 \mathrm{mM}$ leucine for 15 mins (B) or $1 \mu \mathrm{M}$ rapamycin for $1 \mathrm{~h}(\mathrm{C})$. Following treatments, cells were fixed and stained to detect endogenous GSK3 $\beta$. Shown for each (left panels) are micrographs obtained by wide-field epifluorescence microscopy representative of three independent experiments. Shown for each condition as GSK3 $\beta$ overlay are sample cellular and nuclear outlines of single cells. Also shown (right panel) is the mean GSK $3 \beta$ nuclear localization index \pm S.E. (error bars) $(\mathrm{n}=3,>30$ cells/condition/experiment); *, $\mathrm{p}<0.05$ relative to control conditions (absence of EBSS +/- leucine or rapamycin). 
Chapter 6: Discussion 
6.1 Chapter 3: Clathrin plasma membrane nanodomains are signalling platforms for EGF $\underline{\text { stimulated Akt activity }}$

In this part of my thesis, I uncovered that the CME component clathrin plays an important role in controlling EGF-stimulated Akt activation, in epithelial cells in culture derived from a healthy donor (RPE) and in triple-negative breast cancer cells (MDA-MB-231 cells). Importantly, while clathrin was required for EGF-stimulated Akt phosphorylation, this phenomenon does not require receptor endocytosis, of which the final stages are mediated by dynamin2. Using siRNA gene silencing to specifically target the gene of interest in RPE cells, clathrin knock down lead to a significant decrease in Akt activity, while dynamin 2 knock down had no effect (Fig. 3.1). These results obtained by perturbing clathrin suggest that early stages of formation of CCPs and likely CCPs themselves are required and important to regulate Akt activity, while the later stages of CME such as scission of CCPs from the cell surface to produce vesicles by dynamin 2 are not required for signalling to Akt. This novel role for clathrin in controlling EGFR signalling can be seen in different cell lines, as MDA-MB-231 cells showing a similar effect as in RPE cells. Specifically, either pharmacological inhibition of clathrin using pitstop2 or a complementary approach to silencing clathrin with siRNA led to a reduction in EGF-stimulated signalling to Akt phosphorylation (Fig. 3.2), suggesting that the effect of clathrin may control EGF stimulated Akt activity. Interestingly, this effect of clathrin to control signalling upon EGFR activation may be specific to activation of the Akt pathway, as the Erk pathway is not affected after clathrin and dynamin 2 perturbations both in RPE and MDA-MB231 cells (Fig. 3.2). 
The role of clathrin in controlling EGFR signalling may reflect analogous mechanisms by which clathrin regulates GPCR signalling. As shown by Elenko et al. (2003), Delta-Opioid receptor signalling (DOR) requires spatial segregation within CCPs with its signalling intermediate $\mathrm{G} \alpha$-interacting protein (Elenko et al., 2003). After agonist stimulation with [D-Pen2 ,D-Pen5 ]-enkephalin (DPDPE) to DOR, promotes dissociation Gai3 from the inhibitory subunit of heterotrimeric $\mathrm{G}$ proteins, resulting in G-protein signalling activation (Elenko et al., 2003). Blocking endocytosis of DOR through dynamin mutant K44A, prevents Gai3 release from inhibitory subunit of heterotrimeric G proteins (Elenko et al., 2003). Eichel et al. in 2016, also demonstrated that $\beta$-arrestin gets recruited into CCPs even without activating the receptor, which is required for MAPK signalling (Eichel et al., 2018; Eichel et al., 2016). Similar to our results, EGFR recruitment into CCPs is important for regulating Akt activity. Thus, clathrin generates a plasma membrane nanodomain, where clathrin acts as an signalling intermediate or platform to control Akt activity.

In previous reports, Sigismund et al. in 2008 had also shown that silencing of both clathrin and dynamin2 results in decreased activation of Akt and p42/44 MAPK (Erk). This previous study examined prolonged EGFR signalling, at times $>30$ minutes after EGF stimulation (Sigismund et al., 2008). In contrast, my experiments on EGFR signalling focused on acute time points for signalling $(5-10 \mathrm{~min})$ in order to allow selective analysis of receptorproximal signalling regulation. In contrast to their study, I found that Erk was not affected by clathrin perturbation, but that Akt phosphorylation was decreased upon clathrin perturbation, when measured within 10-min (acute) of EGF stimulation. Collectively, our findings suggest that 
clathrin plays more of a direct role at the plasma membrane during acute EGF stimulation for Akt activation, that does not require dynamin2.

Other studies from the Antonescu lab and other labs demonstrate contribution of clathrin in signalling. Garay et al. (2015) demonstrated that CCPs are enriched in phosphorylated Gab1, suggesting that signals cluster within CCPs to trigger specific phosphorylation of Gab1 (Garay et al., 2015). CCPs also harbor Target of Myb1 Like 1 Membrane Trafficking Protein (TOM1L1) and Fyn, which are required for EGF-stimulated Gab1 and Akt phosphorylation (Manuscript in preparation by Lucarelli, S). Furthermore, CCPs that harbor TOM1L1 or Fyn are longer lived, larger and are depleted in some factors such as epsin (Manuscript in preparation by Lucarelli, S). Hence, CCPs that are capable of signalling are different than regular CCPs. Rosselli-Murai et al. in 2018, also demonstrated that PTEN is enriched in some CCPs and controls the lifetime of CCPs, which may modulate signalling (Rosselli-Murai et al., 2018). Furthermore, Rosselli-Murai et al., in 2018, observed enrichment of phosphorylated Akt in some CCPs (Rosselli-Murai et al., 2018). Recently, Pascolutti et al. in 2019, also demonstrated that CCPs, through the control of AP2, regulate Akt activation but not Erk (Pascolutti et al., 2019). Overall, CCPs contribute to the control of signalling, different from endocytosis.

\subsection{Chapter 3: Isoform specific regulation by Dynamin1 controls EGF stimulate Akt} phosphorylation

I had shown previously that clathrin perturbations, but not that of dynamin2, inhibit EGF stimulated Akt phosphorylation (Fig. 3.1). In addition, siRNA gene silencing of clathrin and 
dynamin2 do not affect EGFR phosphorylation (Garay et al., 2015). PLC $\gamma 1$ regulates CME dynamics (Delos Santos et al., 2017), and I showed that PLC $\gamma 1$ is required for EGF-stimulated Akt phosphorylation (Fig. 3.3B). Evidence from a previous paper have demonstrated that PLC $\gamma 1$ may act as a guanyl exchange factor to regulate endocytosis through a different dynamin isoform, dynamin1 (Choi et al., 2004). Furthermore, dynamin1 also selectively regulates TRAIL death receptor endocytosis as well as the apoptosis signalling by this receptor (Reis et al., 2017). This suggested the possibility dynamin 1 activity, either activated by PLC $\gamma 1$ or by other mechanisms such as phosphorylation of GSK3 $\beta$ (Reis et al., 2015) may regulate EGFR endocytosis and signalling activity. My results using dynamin1 siRNA show that perturbation of dynamin1 impacted both EGFR phosphorylation as well as the downstream activation of Akt. Importantly, this effect of dynamin1 perturbation is distinct from the effect or perturbing clathrin, because 1) silencing dynamin1 but not clathrin decreased cell surface EGFR levels (Appendix Fig. A1B), 2) silencing dynamin1 but not clathrin decreased EGF-stimulated EGFR phosphorylation (Fig. 3.4B), and silencing clathrin but not dynamin1 impacted EGFR internalization (Delos Santos et al., 2017). Thus, dynamin1 may promote EGFR recycling, as Lakoduk et al. in 2019, demonstrated that dynamin1 promotes the recruitment of APPL1 to APPL1 endosomes, to promote recycling of some cargo (like EGFR) back to the plasma membrane (Lakoduk et al., 2019). Dynamin1 may regulate EGFR in a manner more consistent with regulation of EGFR recycling rather than control of signalling - specific clathrin nanodomains at the cell surface. 
6.3 Chapter 3: PLC $\gamma 1$ modulates CME dynamics, in turn regulating EGF stimulated Akt activity

After uncovering the importance clathrin as a scaffold for regulating EGFR activity at the plasma membrane, I also found that PLC $\gamma 1$ activity modulates CME dynamics (In collaboration with Dr. R.C. Delos Santos), ultimately controlling EGF-stimulated Akt activation. siRNA gene silencing of PLC $\gamma 1$ lead to impairment of EGF- stimulated Gab1 and Akt phosphorylation, but not EGF-stimulated EGFR phosphorylation (Fig. 3.3B\&C), or Erk phosphorylation (Fig. 3.3C). This suggests that PLC $\gamma 1$ provide a positive feedback mechanism that may selectively modulate the Gab1-PI3K-Akt pathway downstream of EGFR.

PLC $\gamma 1$ activity generates DAG and IP3 from PI(4,5) $\mathrm{P}_{2}$, which are important secondary messengers that bind to PKC and IP3 calcium channels, respectively. IP3 subsequently increases intracellular release of calcium (Nishizuka 1988; Berridge and Irvine, 1984). Calcium is important for activating calcineurin (Saheki and De Camilli, 2012; Cousin and Robinson, 2001; Cousin, 2000), which in turn regulates CME components some including dynamin1, amphiphysin, synaptojanin1, eps15, epsin, and AP180, allowing for these available proteins to increase CME (Saheki and De Camilli, 2012; Cousin and Robinson, 2001; Cousin, 2000; Chen et al., 1999; Slepnev et al., 1998; Bauerfeind et al., 1997; Liu et al., 1994). With these results, it is possible that PLC $\gamma 1$ acts as a primer to enhance the formation of CCPs or their ability to function as signalling platforms. Demonstrated by Delos Santos et al. in 2017, PKC, IP3-receptor, and PLC $\gamma 1$ inhibitions all had the same effect to impair EGFR endocytosis (but not that of TfR) (Delos Santos et al., 2017). Inhibitors of all three inhibited the assembly of clathrin (measured by fluorescence intensity of clathrin within CCPs) in CCPs that have EGFR but not those that have 
TfR (Delos Santos et al., 2017). Thus, PLC $\gamma 1-\mathrm{Ca}^{2+}-\mathrm{PKC}$ controls CCP assembly, which in turn promotes clathrin's role as a scaffold to promote Akt activation.

\subsection{Chapter 4: Mechanism of control of GSK3 $\beta$ nuclear localization by mTORC1}

I found that direct inhibition of any component of the PI3K-Akt-mTORC1 axis, or activation of AMPK to trigger mTORC1 inhibition, results in an increase in GSK3 $\beta$ nuclear localization. Moreover, perturbation of Rab7-dependent membrane traffic also resulted in an increase in GSK3$\beta$ nuclear localization, suggesting that in addition to mTORC1 signals, lysosomal traffic and/or organization is also required to control GSK3 $\beta$ nuclear import. Interestingly, I also observed that inhibition of PI3K-Akt-mTORC1 also increased nuclear localization of GSK $3 \alpha$. Hence, it is likely that mTORC1 signals similarly gate GSK $3 \alpha$ and GSK3 $\beta$ nuclear localization.

GSK3 $\alpha$ nuclear localization was proposed to be uniquely regulated by a calcium- and calpain-mechanism, dependent on its $\mathrm{N}$-terminus cleavage triggering nuclear localization (Azoulay-Alfaguter et al., 2011). However, there was not a detectable lower molecular weight of other GSK3 $\beta$ species in the western blot and nuclear localization by mTORC1 inhibition is reversible (not possible if triggered by cleavage of GSK3 $\beta$ by calpain, which is permanent due to the possible removal of important sequences that dictate localization (Bozóky et al., 2011)). Furthermore, both GSK3 $\alpha$ (Azoulay-Alfaguter et al., 2011) and $\beta$ (Bechard and Dalton, 2009) were reported to undergo nuclear translocation in response to serum withdrawal, consistent with our observations of a central role for mTORC1 in control of GSK3 $\alpha / \beta$ nuclear localization 
which I report here. Taken together, I propose that mTORC1 establishes a form of 'molecular licencing' for retention within the cytoplasm for GSK3 $\alpha$ and GSK3 $\beta$, resulting in nuclear exclusion under conditions of elevated mTORC1 activity. This molecular licencing could take the form of a post-translational modification of GSK3 $\alpha$ and/or GSK3 $\beta$, or of regulation of protein complex formation at specific subcellular locale(s), which I discuss further below.

GSK3 $\beta$ undergoes nucleocytoplasmic shuttling, due to nuclear import in balance with FRAT-1-mediated nuclear export (Franca-Koh et al., 2002). Nuclear import of some (but not all) proteins is controlled by a gradient of GTP -bound and GDP -bound Ran that spans the nuclear membrane (Strambio-De-Castillia et al., 2010). By expression of mutants of Ran (Fig. 4.4), I showed that the nucleocytoplasmic shuttling of GSK3 $\beta$ is Ran-dependent. Moreover, nuclear import of GSK $3 \beta$ resulting from mTORC1 inhibition by rapamycin was prevented in cells expressing Ran G19V mutant defective in GTP hydrolysis and thus defective in nuclear import. Hence, nuclear import of GSK3 $\beta$ regulated by mTORC1 is Ran-dependent.

To address if mTORC1-dependent control of GSK3 $\beta$ nucleocytoplasmic shuttling is mediated by a change in post-translational modification, I examined whether the phosphorylation of S9 on GSK3 $\beta$ could control its mTORC1 -regulated nuclear localization. Two observations strongly suggest that this is not the case: (i) inhibition of mTORC1 by rapamycin did not alter S9 phosphorylation of GSK3 $\beta$ (Fig. 4.6A), yet robustly impacted nuclear localization of GSK3 $\beta$ (Fig 4.3B) and (ii) a mutant of GSK3 $\beta$ that cannot be phosphorylated at this position (S9A) behaved similarly to wild -type with respect to mTORC1-dependent nuclear localization (Fig. 4.8B), similar to a previous report with this GSK3 $\beta$ mutant (Meares and Jope, 2007). 
GSK3 $\beta$ can also be phosphorylated on a number of other residues, including Y216, which may result from autophosphorylation at the time of GSK3 $\beta$ synthesis (Beurel et al., 2015). Further, GSK3 $\beta$ can be phosphorylated at T43 (Ding et al., 2005) and S389 (Thornton et al., 2008) by Erk and p38 MAPK, respectively, each of which lead to reduction in GSK3 $\beta$ activity. Notably, using a phos-tag gel electrophoresis approach, a technique that exacerbates the apparent molecular weight increase caused by phosphorylation, I was only able to resolve two bands for GSK3 $\beta$ that likely correspond to S9 phosphorylated and non - S9 phosphorylated forms (Fig. 4.9). It will be interesting to determine in future studies if and how phosphorylation at sites other than S9 are regulated by mTORC1 to control GSK3 $\beta$ nuclear localization.

Other than phosphorylation, other modifications reported for GSK3 $\beta$ include citrullination (Stadler et al., 2013) and calpain cleavage (Goñi-Oliver et al., 2007). Calpain cleavage was discussed above. Citrullination of R3 and R5 residues within GSK3 $\beta$ is important for nuclear localization (Stadler et al., 2013). However, we observed that mTORC1 controls both GSK3 $\alpha$ and GSK3 $\beta$ nuclear localization, and these two GSK3 paralogs differ at their N-terminus within the region of GSK3$\beta$ that are expected to undergo citrullination. Hence, it appears unlikely to expect that mTORC1 controls citrullination of GSK3 3 as a mechanism of control of its nucleocytoplasmic shuttling. While beyond the scope of this study, it will be interesting to note how future work may resolve whether mTORC1 -dependent regulation of post -translational modification of GSK3 $\beta$ underlies the regulation of its nuclear localization by mTORC1. Currently, I have no strong evidence that any known post-translational modification of GSK3 $\beta$ 
contributes to the regulation of GSK3$\beta$ nucleocytoplasmic shuttling by mTORC1, so perhaps it is an unknown mechanism(s) or control of other facets of GSK3 $\beta$.

mTORC1-dependent control of GSK3 $\beta$ nuclear localization may occur as a result of regulation of GSK3 $\beta$ interaction with other proteins in various endomembrane compartments. It is worth noting that the vast majority of cytoplasmic, but not nuclear GSK3 $\beta$, is associated with other protein(s), which was demonstrated by Meares and Jope, 2007, using membrane permeable ethylene glycol bis (succinimidylsuccinate) followed immunoblotting for GSK3 $\beta$ (Meares and Jope, 2007). Thus, it is possible that control of GSK3 $\beta$ nucleocytoplasmic shuttling involves regulation of protein -protein interactions that serve to occlude the bipartite NLS of GSK3 $\beta$ (residues 85 to 103) (Meares and Jope, 2007), thus limiting GSK3 $\beta$ nuclear localization when these interactions are present.

I also found that Rab7 is required to retain GSK3 $\beta$ in the cytoplasm under conditions when mTORC1 is otherwise active. Importantly, disruption of late endosome/lysosome membrane traffic by perturbations of Rab7 or other proteins does not impact mTORC1 activity (Flinn et al., 2010). This indicates that the ability of mTORC1 to limit the nuclear localization of GSK $3 \beta$ requires active traffic to the late endosome/lysosome. This in turn suggests that the protein interactions engaged by GSK3 $\beta$ that occlude its NLS and thus limit nuclear localization may occur on the lysosome, consistent with our observed localization of GSK3 $\beta$ to the lysosome. Indeed, GSK3 $\alpha$ and GSK3 $\beta$ have nearly identical kinase domains (in which the NLS is found), consistent with the ability of mTORC1 to gate nuclear access for both GSK3 paralogs. 
Furthermore, our observations that mTORC1 controls GSK3 $\beta$ nuclear localization add to previous reports that GSK3 $\beta$ activates mTORC1 signalling (Inoki et al., 2006), and suggests the existence of reciprocal regulation of $\mathrm{mTORC} 1$ and GSK3 $\beta$. Overall, I propose that mTORC1 signals limit the ability of GSK3$\beta$ to localize to the nucleus, and that this may result from mTORC1-dependent control of GSK3 $\beta$ interactions with other proteins in a manner that regulates occlusion of the NLS of GSK3 $\beta$ at the lysosome.

\subsection{Chapter 4: Regulation of GSK3 $\beta$ nuclear functions by mTORC1}

I identified that various metabolic and mitogenic signals gate nuclear access for GSK3 $\beta$. This in turn allows for GSK3 $\beta$-dependent regulation of nuclear substrates in response to mTORC1 signals. Previous studies reported that nuclear and cytoplasmic pools of GSK3 $\beta$ have distinct functions, such as nuclear GSK3 $\beta$ facilitating stem cell differentiation over self-renewal (Bechard and Dalton, 2009) or the cytosolic pool of GSK3 $\beta$ being sufficient to mediate GSK3 $\beta$ dependent cell survival to tumor necrosis factor $\alpha(\mathrm{TNF} \alpha)$ apoptotic signals (Mearse and Jope, 2007).

One of the nuclear substrates of GSK3 $\beta$ is c-Myc, a helix-loop-helix leucine zipper transcription factor that under typical conditions has a very short half-life (15 -30 mins) (Kalkat et al., 2017; Lüscher and Eisenman, 2015). As previously reported, nuclear localization of GSK3 $\beta$ is required for phosphorylation of c-Myc on T58, resulting in enhanced c-Myc degradation (Gregory et al., 2003). I show that rapamycin treatment, which promotes nuclear localization of GSK3 $\beta$, also results in an acute reduction in c-Myc accumulation (Fig. 5.1), most 
likely due to c-Myc degradation. A previous report suggested that rapamycin treatment elicits degradation of c-Myc by induction of autophagy, as result of regulation of AMBRA-dependent dephosphorylation of c-Myc at S62 (Cianfanelli et al., 2015). However, I show that the degradation of c-Myc induced by rapamycin is insensitive to impairment of autophagy induction elicited by siRNA gene silencing of ULK1 (Fig. 4.7). Moreover, I find that the rapamycininduced reduction in c-Myc levels is countered by either by chemical inhibition or silencing of GSK3 $\beta$ (Fig. 5.1A-B). Hence, my results indicate that mTORC1-dependent control of GSK3 $\beta$ nuclear localization regulates c-Myc in a manner that does not require induction of autophagy, and instead likely involves c-Myc degradation subsequent to phosphorylation by GSK3 $\beta$ (Gregory et al., 2003).

Based on the control of GSK3 $\beta$ nuclear localization by mTORC1 leading to control of cMyc, I propose the existence of a metabolic sensing signalling network that links nutrient availability with biomass production and proliferation. Indeed, c-Myc controls the expression of many genes, generally to promote ribosome production, biomass accumulation and enhanced cellular bioenergetics, such as through mitochondrial biosynthesis (Miller et al., 2012). Furthermore, c-Myc promotes epithelial -mesenchymal transition (Cho et al., 2010) and promote proliferation (Dang et al., 2006). c-Myc is involved in many cancers and has been thought to be very difficult to treat (Chen et al., 2018; Darnell 2002). In TNBC, c-Myc has been shown to be essential for driving mitochondrial biogenesis and drug resistance (Lee et al., 2017). In my work, the effect of inhibiting mTORC1 leading to degradation of c-Myc via GSK3 $\beta$ may be a possible route for the development of therapies to perturb c-Myc in cancer. Hence, signals activated during nutrient deficiency can impair the anabolic c-Myc-dependent promotion of biomass 
accumulation via this novel mTORC1 -GSK3 $\beta$ - c-Myc signalling axis involving control of GSK3 $\beta$ nuclear localization.

GSK3 $\beta$ may also regulate other nuclear substrates selectively during conditions of reduced mTORC1 signalling or other states in which GSK3 $\beta$ exhibits nuclear localization. Collectively, regulation of other GSK3 $\beta$ substrates such as Snail (leading to degradation, (Sekiya and Suzuki, 2011)) or c-jun (leading to impaired DNA binding, (Nikolakaki et al., 1993)) is consistent with the effect of GSK3 $\beta$-dependent degradation of c-Myc: reduced cell cycle progression, impairment of epithelial-mesenchymal transition and/or reduced biomass accumulation. While examination of mTORC1-dependent regulation of all known GSK3 $\beta$ nuclear targets is beyond the scope of this study, it is possible to examine targets by using RNAseq (a technique used to measure the mRNA levels of every single gene in a cell sample). In addition, it is perhaps tempting to speculate that metabolic and mitogenic signals broadly control the nuclear profile of GSK3 $\beta$ functions, coordinating energy-demanding accumulation of biomass, cell cycle progression and growth with nutrient availability, but this is beyond the scope of this work.

As cancer cells exhibit heterogeneity of metabolic cues and signals, it is possible that differences in metabolism between cancer cells that result in distinct GSK3 $\beta$ nuclear localization profiles may underlie in part the differences in response to drugs targeting GSK3 $\beta$ in cancer, although this remains to be examined. There is evidence for AMPK and mTORC1 signalling heterogeneity in cell populations. AMPK phosphorylation responds differently for myocytes and 
hepatocytes after AICAR or metformin treatment (Konagaya et al., 2017). Furthermore, Human Bone Osteosarcoma Epithelial Cells exposed to modest cell stress ( $3 \mathrm{mM}$ of glucose), showed cell to cell variance in AMPK activity (Tsou et al., 2011). Growth factor stimulation activated wide range of activities for mTORC1 at different locations within the cell including plasma membrane, nucleus, and lysosome (Zhou et al., 2015). AMPK and mTORC1 activity is heterogeneous, and since each of these control GSK3 $\beta$ nuclear localization, it may be possible for heterogeneity of GSK3 $\beta$ localization.

6.6 Chapter 5: Localization of GSK3 $\beta$ to multiple membrane compartments within the cytoplasm

Separate studies have reported that GSK3$\beta$ may localize to a number of distinct cellular compartments, including endomembranes, mitochondria and the nucleus (reviewed by Beurel et al., 2015). By a systematic, unbiased approach to study several different endocytic compartments, we find that endogenous GSK3 $\beta$ localizes to several distinct such endosomal compartments, including APPL1 endosomes, EEA1-positive early endosomes and LAMP1 positive late endosomes/lysosomes (Fig. 5.1). In each case, the overlap of GSK3 $\beta$ immunofluorescence signal and that of each compartment marker is clearly limited and partial, with substantial proportions of each signal not exhibiting overlap (Fig. 5.1A - C). However, systematic and unbiased analysis of colocalization performed by Manders' (Fig. 5.1A - C) or Pearson's (Fig. 5.2A) coefficient analysis indicates that GSK3 $\beta$ overlap with each compartment is specific and non-random. Previous studies found that GSK3 $\beta$ gets trapped in MVBs and degraded upon activation of Wnt signalling (Vinyoles et al., 2014; Taelman et al., 2010). Considering that GSK3 $\beta$ (observed by spinning disc, that does not offer possibility of resolving 
interior vs surface of MVBs/lysosome) could be internal (e.g. being degraded) or surface (involved in signalling using lysosomes as a platform), SIM was used, which was done in collaboration with Yip C, Kim P, and Vissa, A (U of T and SickKids). The specific recruitment of GSK3 3 to the limiting membrane of LAMP1 -positive late endosomes/lysosomes is supported by images obtained by SIM and unbiased analysis of the localization of GSK3 $\beta$ within the lysosome (Fig. 5.1D), as well as by the observation that perturbation of late endosome/lysosome membrane traffic by expression of a dominant interfering mutant of Rab7 abolishes the overlap of GSK3 $\beta$ with LAMP1 signals (Fig. 5.2C).

My observations are thus consistent with the notion that GSK3 $\beta$ is localized to a number of distinct cellular compartments, with a minor pool that in some cases is $<10 \%$ of total cellular GSK3 $\beta$ recruited to each such compartment at steady state. Specifically, our observations are consistent with previous studies showing GSK3 $\beta$ localization to APPL1 endosomes (Schenck et al., 2008). APPL1 is recruited to a subset of intracellular membrane compartments formed by clathrin-mediated endocytosis. The acquisition of APPL1 by internalized vesicles precedes the acquisition of markers of the EEA1 early endosome (Zoncu et al., 2009). This pool of GSK3 $\beta$ within APPL1 endosomes may be specifically targeted by phosphorylation on S9 by Akt, as silencing of APPL1 abolishes Akt-dependent GSK3 $\beta$ phosphorylation (Reis et al., 2015; Schenck et al., 2008). Notably, I uncovered that perturbation of APPL1 by silencing did not impact mTORC1-dependent control of GSK3 $\beta$ nuclear localization (Fig. 5.3B), suggesting that the APPL1-localized pool of GSK3 $\beta$ does not directly participate in the regulation of GSK3 $\beta$ nuclear localization by mTORC1. 
As mTORC1 localizes to the surface of late endosomes and lysosomes, the pool of GSK $3 \beta$ on these membranes may be under the direct regulation by mTORC1 to control GSK3 $\beta$ nucleocytoplasmic shuttling. Indeed, a previous report had observed some overlap of GSK3 $\beta$ and the lysosome (Li et al., 2016). However, GSK3 $\beta$ may also be sequestered within intraluminal vesicles of multivesicular bodies in response to Wnt signalling (Taelman et al., 2010), raising the possibility that the overlap that we observed by spinning disc confocal microscopy between LAMP1 -positive structures and GSK3 $\beta$ (Fig. 5.1C) could reflect GSK3 $\beta$ within intraluminal vesicles. However, quantification of SIM images suggests that GSK3 $\beta$ at lysosomes is preferentially associated with the limiting membrane of these organelles, and not the lumen (Fig. 5.1D). Moreover, perturbation of Rab7 disrupts the localization of GSK3 $\beta$ and LAMP1 (Fig. 5.2C), yet Rab7 disruption does not impact the sequestration of material into intraluminal vesicles (Vanlandingham and Ceresa, 2009). My results thus add systematic analysis and quantification to indicate that a pool of GSK3 $\beta$ is present on the limiting membrane of the lysosome. My results also suggests that this pool may be subject to regulation by mTORC1, resulting in control of GSK3 $\beta$ nuclear localization.

\subsection{Chapter 5: Amino acid sensing of GATOR1 subunit DEPDC5 controls GSK3 $\beta$ nuclear} localization

mTORC1 activity is regulated by the abundance of amino acids. Consistent with regulation of GSK3 $\beta$ nuclear localization by mTORC1, I uncovered that in cells deprived of amino acids, GSK3 $\beta$ translocates into the nucleus (Fig. 4.5C). This suggests a role for 
metabolism to dynamically control GSK3 $\beta$ localization through mTORC1. In addition, populations of GSK3 $\beta$ is localized to the lysosome as shown by both the confocal and SIM images (Fig. 5.1 C\&D), suggesting that the lysosome may be a signalling platform to receive signals from mTORC1 to control its lysosome localization.

Using several other strategies, I showed that amino acids play a significant role in controlling the dynamic nuclear localization of GSK3 $\beta$. Cells starved of amino acids exhibited strong nuclear localization of GSK3 $\beta$, while subsequent treatment with leucine or arginine alone, or the combination of nonessential amino acids with EGF, promoted the export of GSK3 $\beta$ out of the nucleus (Fig. 5.4). This suggests a strong role for amino acid sensing to control the nuclear export of GSK3 $\beta$.

Furthermore, I identified that DEPDC5, the key subunit of the important lysosomal amino acid sensor complex, GATOR1, is a candidate for strong interaction with GSK3 $\alpha$ and $\beta$ through BioID studies. I showed that DEPDC5 controls GSK3 $\beta$ dynamic localization to lysosomes, as cells with a DEPDC5 KO exhibited a decrease in GSK3 $\beta$ lysosomal residence (Fig. 5.5A), suggesting a role for DEPDC5 to tether GSK3 to the lysosomal surface. GATOR1 is negative regulator of mTORC1 activity, and is suppressed by GATOR2 upon sensing of specific amino acids, leading to activation of mTORC1 (as reviewed by Saxton and Sabatini, 2017). I uncovered that cells with knock out of DEPDC5 lose amino acid sensing ability, as GSK3 $\beta$ no longer translocates into the nucleus after DEPDC5 KO cells are deprived of amino acids (Fig. 5.5B). Furthermore, loss of DEPDC5 leads to strong upregulation of mTORC1 activity, as shown by S6K hyperphosphorylation (Appendix Fig. A4). mTORC1 may localize to other 
compartments in the cell (Betz and Hall, 2013) and this mechanism of DEPDC5 dependent tethering of GSK3 3 to the lysosome, may relieve the need to have GSK3 $\beta$ and mTORC1 to meet on endosomes. Thus, two parallel pathways may control GSK3 $\beta$ nuclear localization: 1) dependent on mTORC1 and 2) DEPDC5 binding and tethering to the lysosome.

Amino acid availability also influences the dynamic localization of other transcription factors such as TFEB. Under mTORC1 inhibited conditions (e.g. nutrient deprivation), TFEB is translocated into the nucleus, therefore leads to the upregulation of lysosomal biogenesis (Bajaj et al., 2018; Puertollano et al., 2018). Recently, according to Napolitano et al. in 2018, the effect of amino acid replenishment leading to export of TFEB out of the nucleus is due to regulation of TFEB by mTORC1 phosphorylation (Napolitano et al., 2018). Many reports have observed mTORC1 localization in the nucleus (Audet-Walsh et al., 2017; Betz and Hall, 2013; Yadav et al., 2013; Shi et al., 2010; Tsang et al., 2010; Rosner et al., 2007). However, many reports observe only detectable subunits (mTOR and Raptor) in the nucleus (Audet-Walsh et al., 2017; Betz and Hall, 2013; Shi et al., 2010; Tsang et al., 2010), but not intact mTORC1 (Rosner and Hengstschläger, 2008). Furthermore, Yadav et al. in 2013 demonstrated that both mTOR and Rheb GTPase localize in the nucleus together (Yadav et al., 2013). Thus, it is possible that amino acid replenishment by leucine to DEPDC5 and EGF stimulation, may be mediating nuclear export of GSK3 $\beta$ by controlling nuclear localization of mTORC1 (Fig. 5.4 and 5.5B).

Non-essential amino acids may positively regulate mTORC1 activity as well as the more well-understood leucine and arginine. Jewell et al., in 2015 demonstrated that cells in the 
presence of non-essential amino acid glutamine, enhances mTORC1 activity but with the requirement of functional V-ATPase (Jewell et al., 2015). Furthermore, they show that simultaneous signalling from both mitogenic (insulin) and metabolic (amino acids) pathways, synergistically up regulate mTORC1 activity (Jewell et al., 2015). Similarly, stimulating cells simultaneously with non-essential amino acids (metabolic signals) and EGF (mitogenic signals), may synergistically activate nuclear mTORC1, to export GSK3 $\beta$ out of the nucleus (Fig. 5.4C). 


\section{$\underline{6.8 \text { Future Directions }}$}

My research thus far has demonstrated that mitogenic signalling to EGFR requires clathrin as a scaffold to act as nanodomains for signalling towards Akt, while dynamin1 acts to regulate EGFR recycling (Chapter 3). Furthermore, mitogenic and metabolic signalling controls mTORC1 activity regulating function and subcellular nucleocytoplasmic shuttling of GSK3 (Chapter 4). Finally, my research also shows that lysosomal organization through membrane traffic and DEPDC5 protein tether is important to control GSK3 nucleocytoplasmic shuttling and lysosomal localization, respectably (Chapter 5).

mTORC1 is localized and activated at lysosomes (Saxton and Sabatini, 2017; Efeyan et al., 2012; Sancak et al., 2010). I have uncovered that populations of GSK3 $\beta$ are localized to the surface of the lysosome (Fig. 5.1C\&D) and perturbation of the lysosome using mutant Rab7 leads to translocation of GSK $3 \beta$ into the nucleus, and subsequent degradation of transcription factors c-Myc (Fig. 5.3C). Thus, the mechanisms underlying how the lysosome contributes to GSK3 membrane recruitment are important to understand how these contribute to regulation of GSK3 nuclear localization and transcriptional reprogramming.

DEPDC5 is a GATOR1 subunit that contributes to the amino acid sensor and lysosomal surface protein that regulates mTORC1 activity (Shen et al., 2019; Chantranupong et al., 2016; Saxton et al., 2016; Chantranupong et al., 2014; Parmigiani et al., 2014). In collaboration with the Gingras lab, a proximity biotinylation assay (BioID) identified DEPDC5 as a strong 
interacting partner with both GSK3 $\alpha$ and $\beta$. Furthermore, I found that DEPDC5 KO leads to decreased GSK3 3 localization to the lysosomal surface (Fig. 5.5A) and insensitivity of GSK3 $\beta$ nuclear localization to amino acid deprivation (Fig. 5.5B). Thus, I have shown a part of potential molecular mechanism of how DEPDC5 recruits GSK3 to the lysosome, allowing for mTORC1 to control GSK3 $\alpha / \beta$ nuclear translocation.

From my work emerges some important new questions: 1) How does DEPDC5 recruit GSK3 to the lysosome, 2) How does regulation of GSK3 nuclear translocation control cancer metabolism, growth, and invasion, and 3) How might regulation of GSK3 $\beta$ nuclear localization be exploited for the development of novel therapies aimed at c-Myc-dependent cancers or to target metabolic vulnerabilities in cancer. I hypothesize that a specific domain of DEPDC5 is required to tether GSK3 to the lysosome, an interaction which is important for either 1) mTORC1 dependent control of GSK3 nucleocytoplasmic shuttling, or 2) a putative direct regulation of nuclear export of GSK3 $\beta$ by leucine and arginine, perhaps via Castor/Sestrin sensing through GATOR2 into GATOR1, or both. These signals thus modulate GSK3 nuclear localization to control GSK3 function in cancer cells for invasion, stemness, proliferation, and response to pharmacological treatments in triple negative breast cancer (TNBC). Specifically, I hypothesize that TNBC shift towards a cytosolic GSK3, allowing transcription factors like cMyc or Snail to control metabolic reprograming, cancer invasion, stemness, and drug sensitivity. To test this hypothesis, the objectives for future directions will be as follows:

Determine how DEPDC5 recruits GSK3 $\beta$ to the surface of the lysosome. Proximity biotinylation results suggest that DEPDC5 strongly interacts with GSK3 but does not mention 
how GSK $3 \alpha / \beta$ may specifically bind to DEPDC5. Since loss of DEPDC5 leads to robust and aberrant activation of $\mathrm{mTORC} 1$ through its GAP domain, identification of the specific regions of DEPDC5 that interact with GSK3 $\beta$ may allow resolution of these two activities of DEPDC5. In turn, this would allow understanding of how control of GSK3$\beta$ to the surface of the lysosome impacts GSK3 $\beta$ nucleocytoplasmic conditions under conditions of normal mTORC1 regulation. Thus, the mechanism of how DEPDC5 binds to GSK3 $\beta$ must be elucidated.

Identify the molecular mechanism of how DEPDC5 interacts with GSK3. In order to understand how DEPDC5 and GSK3 interact, site-direct mutagenesis will be used to generate mutants of DEPDC5 that have either deletions of entire domains of DEPDC5 or mutations of specific residues of DEPDC5. The structure of DEPDC5 contains important domains including: N-terminal domain (NTD), SABA domain, Shen domain, DEP domain, LassoA-B regions, and C-terminal domain (CTD) (Shen et al., 2018). NTD, SABA, Shen, DEP and CTD coordinate interactions to being to GATOR1 complex, while LassoA-B regions currently have unknown function (Shen et al., 2018). I look to mutate/truncate domains of DEPDC5, generating $\triangle N T D$, $\Delta \mathrm{SABA}, \Delta \mathrm{Shen}, \Delta \mathrm{DEP}, \Delta \mathrm{LassoA}, \Delta \mathrm{LassoB}$, and $\Delta \mathrm{CTD}$. Once these mutants and truncations have been generated, these can be used for expression in cells, which can be tested for the ability of each to mediate GSK $3 \alpha / \beta$ for interaction. To test the interaction, a proximity biotinylation assay will be used, as each mutant of DEPDC5 will be fused to a BirA* domain (allowing for BioID), followed by expression in HEK293 cells that with knockout of endogenous DEPDC5 (DEPDC5 KO, as per Fig 5.5 A-C) (Fig. 6.1). Each sample will be subject to isolation of biotinylated proteins by streptavidin pull-down, followed by and immunoblotting to detect GSK3 $\alpha / \beta$. As shown by the architecture of DEPDC5 (Shen et al., 2018) (Fig. 6.1), different 
mutants/truncations will be generated for DEPDC5, which may impair interacting and binding to GSK3, thus will be confirmed using co-immunoprecipitation. These experiments will elucidate how the different motifs or domains will be required for DEPDC5 to bind to GSK $3 \alpha / \beta$. I will also test mTORC1 activity in each and I expect to identify at least a mutant/truncation that lose GSK $3 \alpha / \beta$ interaction but retain suppression of mTORC1 activity.

To follow up these experiments, I next propose characterizing how each different mutants/truncations of DEPDC5 that impairs GSK3 interaction affects 1) GSK3 $\alpha / \beta$ localization to the lysosome, 2) mTORC1-dependent GSK3 nucleocytoplasmic shuttling, and 3) leucine and arginine-dependent regulation of GSK3 3 nuclear export. To do so, a similar knockout-and-recue approach will be used which involves experiments with DEPDC5 KO HEK293 cells (knockout), engineered to stably express the different mutants/truncated DEPDC5 (see above, rescue), followed by assessment of localization of GSK3. I will also test the regulation of GSK3 $\alpha / \beta$ nucleocytoplasmic shuttling by mTORC1 and possibly separate GSK $3 \alpha / \beta$ export by leucine/arginine, which may be mTORC1 independent, but is likely to be DEPDC5-dependent. These experiments will demonstrate the importance of DEPDC5 as a lysosomal tether to present GSK3 to mTORC1 for alterations in nuclear translocation, and how this coordinates signalling by mTORC1 or direct control by DEPDC5 to gate nuclear GSK3 $\beta$.

Characterize DEPDC5-GSK3 interaction for functional output of nuclear GSK3. Next, to understand the functional outcome of DEPDC5-GSK3 interaction, experiments will use knockout and rescue experiments to impair DEPDC-GSK3 interaction in TNBC MDA-MB-231 cells. Using CRISPR/Cas9 genome editing, DEPDC5 will be knocked out in MDA-MB-231 cells 
and transfected with DEPDC5 mutants that lack interaction with GSK3 $\beta$ but retain suppression of mTORC1, identified above (rescue) (Fig. 6.1). These cells will be used for a number of assays that seek to assess the impact of these perturbations on cancer cell physiology. First, I propose to asses how perturbations of DEPDC5 by this knockout-rescue approach alter expression of c-Myc an Snail. I expect that these experiments will show that impaired interaction between GSK3 and DEPDC5 (under conditions where mTORC1 remains active) will promote GSK3 nuclear localization and functionally decrease both c-Myc and Snail expression. Thus, these future directions may provide a potential mechanism for controlling GSK3 recruitment to the lysosomal, in which DEPDC5 acts as a lysosomal tether but also acting as a "presenter" towards mTORC1 for regulation of GSK3 nuclear localization and function.

Determine how GSK3 nuclear translocation controls cancer metabolism, growth, and invasion. The results presented in Figure 5.3C suggest that mTORC1 interfaces with control of transcription, in part via mTORC1-dependent control of GSK3 nuclear translocation to regulate c-Myc expression. Thus, it is important to understand how the nuclear actions of GSK3 function to control metabolism, cancer cell invasion, and stemness during tumor initiation and metabolic reprogramming in TNBC as well as other cancers, which I propose to do as follows:

Determine how nucleocytoplasmic translocation impacts GSK3 control of cancer cell proliferation, growth, and stemness. In order to understand how the regulation of localization of GSK3 impacts cancer growth proliferation, and stemness, another series of knockout and rescue experiments will be conducted in MDA-MB-231 cells in which GSK3 $\beta$ has been knocked out (which I have established, see Fig. 6.2). These cells will be transfected with GSK3 $\beta$ constructs 
engineered to contain either nuclear localization or nuclear export sequences (Meares and Jope, 2007) (Fig. 6.2). I expect that these cells will exclusively have either nuclear or cytoplasmic GSK3 $\beta$, allowing robust extermination to determine how this localization controls cancer cell physiology.

First, these engineered GSK3 $\beta$ cell lines will be assessed for proliferation/survival using XTT (cell proliferation/survival) assays, BrdU cell cycle progression (proliferation), and caspase cleavage (survival, apoptosis). I also propose to study how each GSK3 $\beta$ engineered cell line exhibits changes in cell migration and invasion, using transwell or scratch wound closure assays (Kramer et al., 2013). Each of these experiments using these engineered GSK3 $\beta$ cell lines will be conducted under different nutrient conditions that span a range of mTORC1 activation states, allowing resolution of how unique nutrient conditions impact cell physiology via control of GSK3 $\beta$ nuclear localization vs other metabolic signals/cues.

Importantly, GSK3 $\beta$ translocation to the nucleus controls c-Myc expression, and c-Myc is a mediator for establishing cancer stem cells (CSC) (Klauber-DeMore et al., 2018; Lee et al., 2017; Yang et al., 2017). Using these engineered GSK3 $\beta$ cell lines, I propose to determine the importance of inclusion or restriction of GSK3 3 to/from the nucleus on CSC formation. This will be monitored by mammosphere formation assessed by flow cytometry (scored via CD44 $/ \mathrm{CD} 24$ and $\mathrm{ALDH}^{+}$cell populations) (O'Connor et al., 2019). I expect that cells expressing only GSK3 $\beta$ restricted to the nucleus will exhibit defects in breast cancer stem cell maintenance as well as impaired growth and proliferation. Thus, these experiments will reveal mechanisms of how GSK3 dynamic nuclear translocation plays an important role in cancer cell physiology. 
Determine how GSK3 nuclear translocation leads to alterations in cancer metabolic reprogramming. Using GSK3 $\beta$ (KO) cells or cells with GSK3 $\beta$ KO also engineered to express GSK3 $\beta$ constructs for either nuclear excluded or nuclear restriction, I propose to use RNA-seq to systematically study how each condition regulates expression of all genes (Hwang et al., 2018). As shown from our findings (Fig. 4.1 A-C), nuclear GSK3 $\beta$ promotes degradation of c-Myc and Snail, but also regulates many other transcription factors. c-Myc controls 100's of targets depending on the cell context, including genes found in oxidative phosphorylation and in glycolysis, processes often dysregulated in many cancers (Stine et al., 2015; Miller et la., 2012). In these experiments, I expect that cells that exclude GSK3 $\beta$ from the nucleus may enhance transcription of genes that promote oxidative phosphorylation and glycolysis, while cells restricting GSK3 $\beta$ to the nucleus should repress transcriptional activity for oxidative phosphorylation and glycolysis. Follow up and complementary experiments to confirm RNA-seq information include LC-MS/MS for quantitative analysis of different metabolites found in glycolysis (Krebs cycle and amino acid metabolism), as well as measurement of mitochondrial membrane potential (through TMRE (Ghosh et al., 2016)), production of reactive oxygen species (Ghosh et al., 2016), and oxygen consumption and lactate production (Hulea et al., 2018). Information from this line of investigation will reveal the importance of compartmentalization from cytosol to nucleus of GSK3 $\beta$ to control metabolic reprogramming in glycolysis and oxidative phosphorylation within TNBC cells.

Determine how GSK3 localization affects cancer drug and treatment response. TNBCs and many cancers reprogram metabolism, or exhibit metabolic adaptation and versatility, which 
in turn impairs the response to different inhibitors and treatments, including specific chemotherapeutic agents (e.g. doxorubicin (Lee et al., 2017)), tyrosine kinase inhibitors (TKIs, e.g. lapatinib (Hulea et al., 2018)), or metabolic inhibitors (e.g. biguanides (Hulea et al., 2018). It is not fully understood how mTORC1 control of GSK3 or the reciprocal regulation (AzoulayAlfaguter et al., 2015; Stretton et al., 2015; Ka et al., 2014) can contribute to cancer cell metabolic adaptation and thus mediate resistance to specific cancer drugs, which I propose to study next, as follows:

Determine how GSK3 nuclear compartmentalization controls TNBC cells treated with different cancer drugs. I propose to use MDA-MB-231 cells harboring knock out GSK3 $\beta$ and engineered to express GSK3$\beta$ constructs that either restrict or exclude GSK3 $\beta$ of the nucleus, to examine the effects of metabolic inhibitors (e.g. phenformin), chemotherapy (e.g. cisplatin, paclitaxel, doxorubicin), and TKIs on cell growth and survival using assays such as XTT (cell proliferation/survival) assays, BrdU (proliferation), migration and invasion assays using transwell or scratch wound closure assays (Kramer et al., 2013). Since GSK3 $\beta$ nuclear localization leads to c-Myc degradation, and increased c-Myc in TNBC is required for the development of resistance to chemotherapy drugs (Lee et al., 2017), I expect that these experiments will reveal that cells that express only GSK3 $\beta$ restricted to the nucleus will exhibit enhanced sensitivity to various cancer drugs, while nuclear exclusion of GSK3 $\beta$ will enhance cancer cell survival. Hence, these experiments will establish control of GSK3 $\beta$ nuclear localization, as occurs via mTORC1 and/or DEPDC5 signalling arms, as an important signalling axis to promote cancer cell adaption in response to drug inhibitor or treatment, thus leading to the development of drug resistance. 


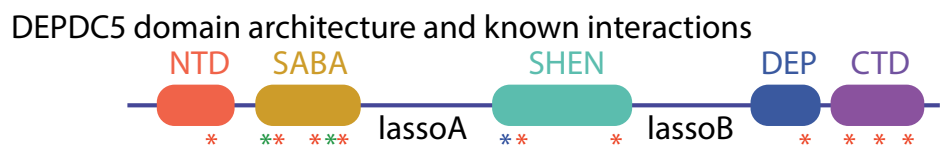

* intramol.interactions * GATOR1 complex interactions * Rag interactions

Potential truncations to probe for GSK3 $\alpha / \beta$ interactions:

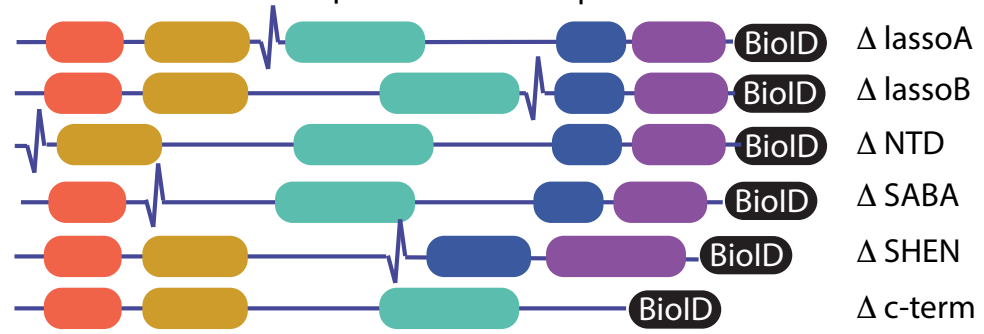

Figure 6. 1 DEPDC5 protein structure and potential mutations/truncations to identify GSK3 $\alpha / \boldsymbol{\beta}$ interactions. Shown (top) is the protein domain structure of GATOR1 subunit, DEPDC5 (Shen et al., 2018). Schematic also includes DEPDC5 sites of interactions towards other substrates: DEPDC5 domains (intramolecular), Rag GTPase (DEPDC5 GAP for Rags), and with NPRL2/3 (GATOR1 complex). Future directions aims to generate truncations in DEPDC 5 and probe for important interactions with GSK $3 \alpha / \beta$ using biotinylation assay, BioID. LassoA-B regions within DEPDC5 current do not have known interactions. NTD, SABA, Shen, DEP and CTD form interactions to generate the GATOR1 complex, regulation of mTORC1, and lysosomal binding. 

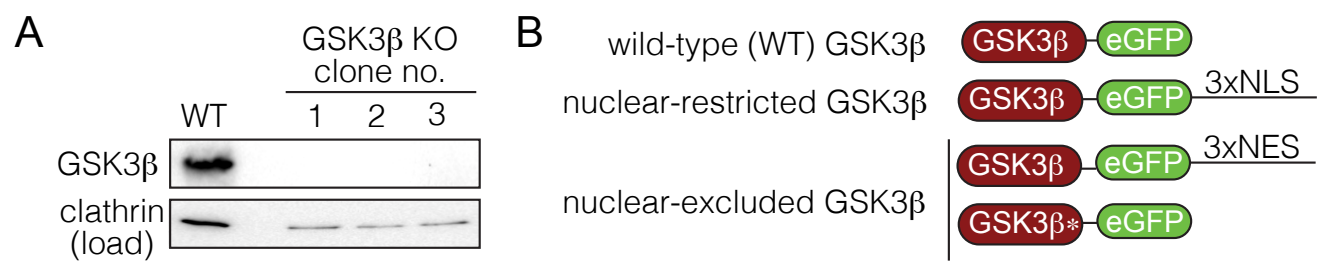

Figure 6. 2 CRISPR/Cas9 knockout of GSK3ß in MDA-MB-231 breast cancer cells and rescue assay. (A) Shown is a representative immunoblots of MDA-MB231 breast cancer cells that are knocked out of GSK3 $\beta$ using CRISPR/Cas9 from Dharmacon. (B) Shown are potential "rescue" GSK3 $\beta$ constructs that will be used for knockout/rescue experiments using MDA-MB-231 GSK3 $\beta$ knockout cells. Different fusion GSK $3 \beta$ constructs will be generated containing nuclear localization sequence (NLS) or nuclear export sequences (NES), which have been described previously by Stadler et al., 2013, Bechard et al., 2009, and Meares et al., 2007. Studies have yet to understand the importance of nuclear localization of GSK $3 \beta$ in terms of cancer cell metabolism. Attached to constructs will be a eGFP tag. 
Conclusion 
In conclusion, my doctoral research uncovered that mitogenic signalling (EGFR-PI3KAkt) is controlled by clathrin signalling domains at the plasma membrane, likely by acting as molecular scaffolds for specific signalling intermediates. I also found that EGFR signalling to PLC $\gamma 1$ also contributes to mitogenic signalling by modulating CME dynamics. Moreover, I discovered that regulation of mitogenic signalling by clathrin and PLC $\gamma 1$ specifically control PI3K-Akt pathway but not Erk signalling, in multiple cell lines such as RPE and MDA-MB-231 cells. Thus, these results uncover a new role for clathrin and clathrin-coated pits, different from scaffolds to mediate plasma membrane internalization events, for the direct regulation of mitogenic signalling through EGFR-PI3K-Akt at the plasma membrane.

I also showed that GSK3 $\beta$ function and localization is regulated by mTORC1, a master regulator of cell physiology. Both mitogenic (EGFR-PI3K-Akt) and metabolic (amino acid availability and energy sensing) signalling regulate mTORC1 activity, which in turn promotes GSK3 $\beta$ retention in the cytoplasm. As such, when mTORC1 is inhibited, GSK3 $\beta$ exhibits nuclear localization and activation of its nuclear programming, leading to the degradation of transcription factors c-Myc and Snail. The mechanism by which GSK3 $\beta$ nuclear localization is regulated by mTORC1 does not depend on regulation of GSK3 $\beta$ phosphorylation. Thus, my results reveal a new mechanism that involves both mitogenic and metabolic signalling that integrate onto mTORC1, controlling GSK3 $\beta$ subcellular localization and nuclear function.

Finally, I uncovered that the lysosome regulates the activity and membrane compartmentalization of GSK3 $\beta$. Rab7 not only regulates the organization of the lysosome, but similarly to $\mathrm{mTORC} 1$ is required for normal control of the nucleocytoplasmic translocation and 
nuclear function of GSK3 $\beta$. In addition, I have uncovered that the lysosomal component DEPDC5 (part of the GATOR1 complex) may tethers GSK3 $\beta$ to the surface of the lysosome and may also sense specific amino acids that in turn control GSK3 $\beta$ nuclear export in a manner that might be independent of (or in parallel of) mTORC1 control of GSK3 $\beta$. Thus, these results reveal a new model for localization of GSK3 $\beta$ at the lysosome and how lysosomal localization of GSK3 $\beta$ dictates the control of GSK3 $\beta$ nuclear localization amino acid availability by DEPDC5.

Overall, the information from this research reveals a new unique signalling axis that integrates mitogenic (EGFR-PI3K-Akt) and metabolic (amino acids and energy) signalling through mTORC1 and/or DEPDC5 to control the function of GSK3 $\beta$. These parallel pathways control the function of GSK3 $\beta$ by controlling its nuclear program through regulation of its transcription factor targets c-Myc and Snail (and perhaps others). This regulation is important, as both transcription factors are dysregulated in cancers, which contributes to reprogramming of cancer cell metabolism and/or EMT, which in turn may allow cancer cells to develop resistance to chemotherapies and drug treatments (Lee et al., 2017; Nawneet et al., 2009). Thus, clinical research and studies may look to use mTORC1-GSK3 $\beta$ signalling axis as an alternative target to strategically regulate cancer genes c-Myc and Snail, presenting a new therapeutic and treatment route for cancer. 
Appendix 

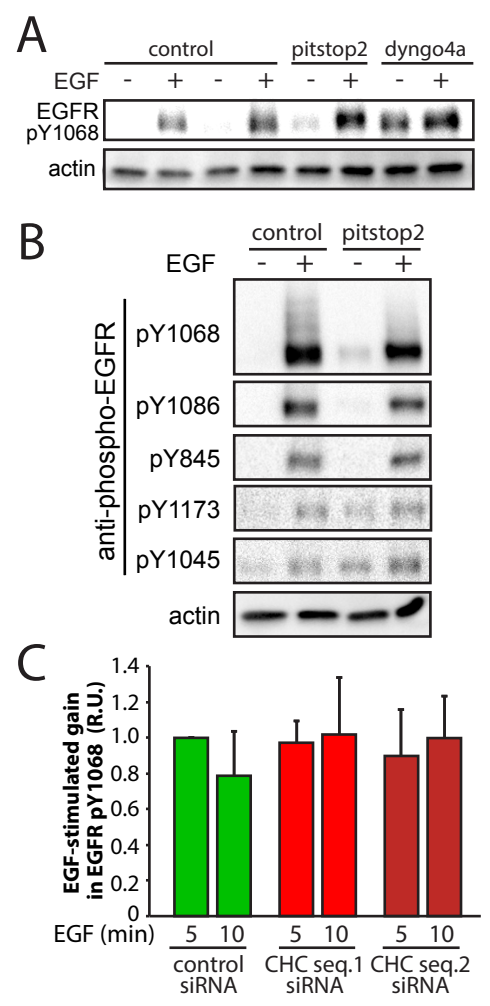

Figure A. 1 Clathrin perturbations does not impair EGFR autophosphorylation. (A\&B) ARPE-19 cells were treated with pitstop2 $(10 \mu \mathrm{M})$, dynago4a $(30 \mu \mathrm{M})$ or vehicle control $(0.1 \%$ [vol/vol] DMSO) for 30 minutes. (C) ARPE-19 cells transfected with siRNA against nontargeting siRNA (control), clathrin siRNA sequence 1 (CHC seq. 1 siRNA), or clathrin siRNA sequence 2 (CHC seq. 2 siRNA). (A-C) After drug treatment or siRNA transfection, ARPE-19 cells were stimulated with EGF $(5 \mathrm{ng} / \mathrm{ml})$ for 5 minutes or unstimulated (basal). $(A \& B)$ Shown are representative immunoblots images of whole-cell lysates probed for antipTyr1068-EGFR, anti-pTyr1086-EGFR, anti-pTyr845-EGFR, anti-pTyr1173-EGFR, antipTyr1045-EGFR, and anti-total actin antibodies. (C) Shown are mean \pm SE of anti-pTyr1068EGFR values; $n=3$. 

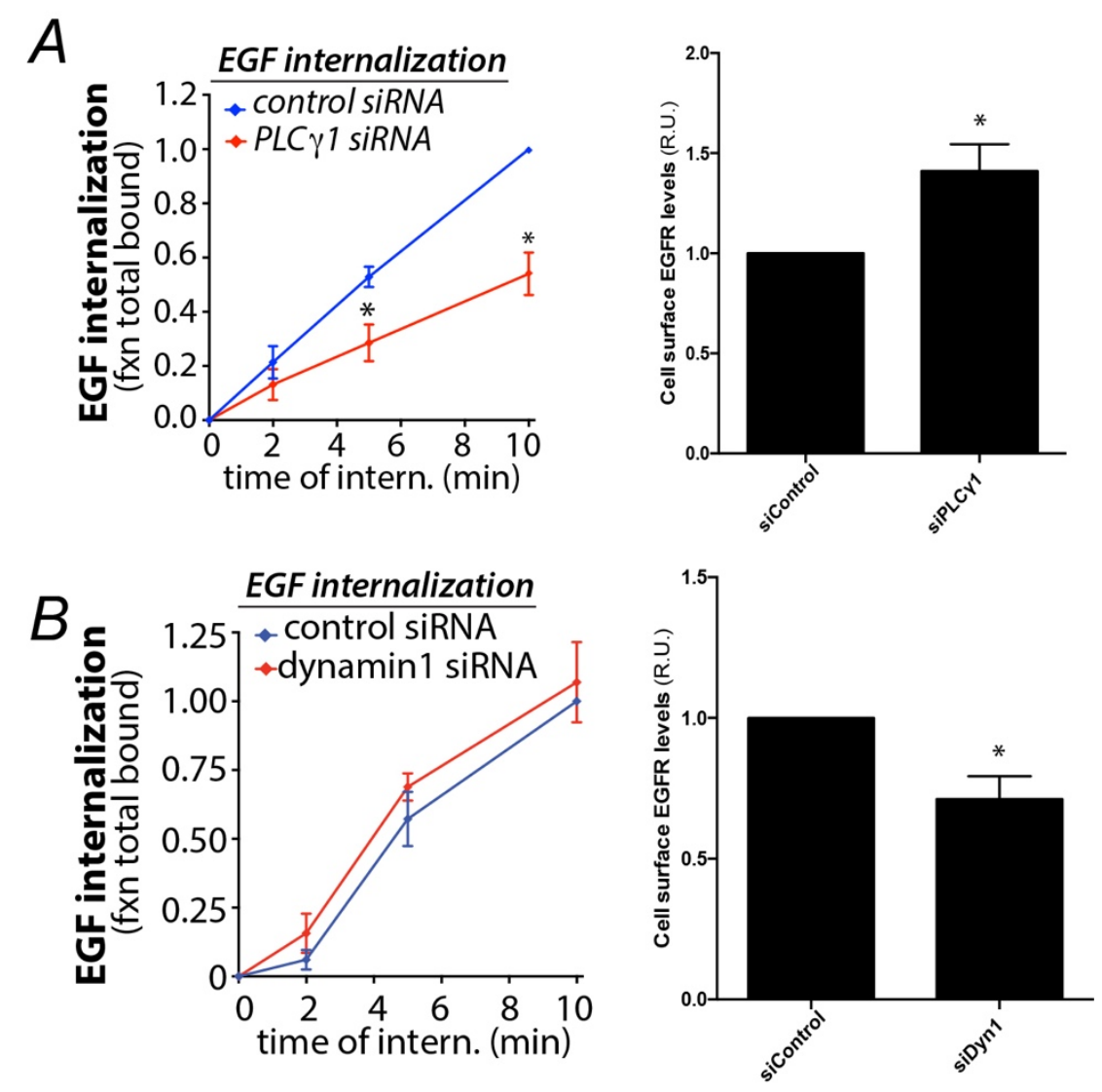

Figure A. 2 PLC $\gamma 1$ siRNA perturbs EGFR internalization but increases cell surface EGFR, while dynamin1 siRNA does not alter internalization but decreases cell surface EGFR. Shown are experiments done in collaborations with Dr. R.C. Delos Santos demonstrating alterations in internalization and EGFR cell surface levels for PLC $\gamma 1$ and dynamin1 siRNA. (A\&B) ARPE-19 cells were transfected using siRNA against nontargeting siRNA (control), PLC $\gamma 1$, and dynamin1. (A\&B left panels) ARPE-19 cells were treated with EGF then followed by measurement of EGF internalization. Shown are the mean \pm SE for $n>3$ independent experiments; *, $p<0.05$. (A\&B right panels) ARPE-19 cell were measured for cell surface levels of EGFR after siRNA transfection. Shown are mean \pm SE for $\mathrm{n}>5$ independent experiments; *, $\mathrm{p}<0.05$. 


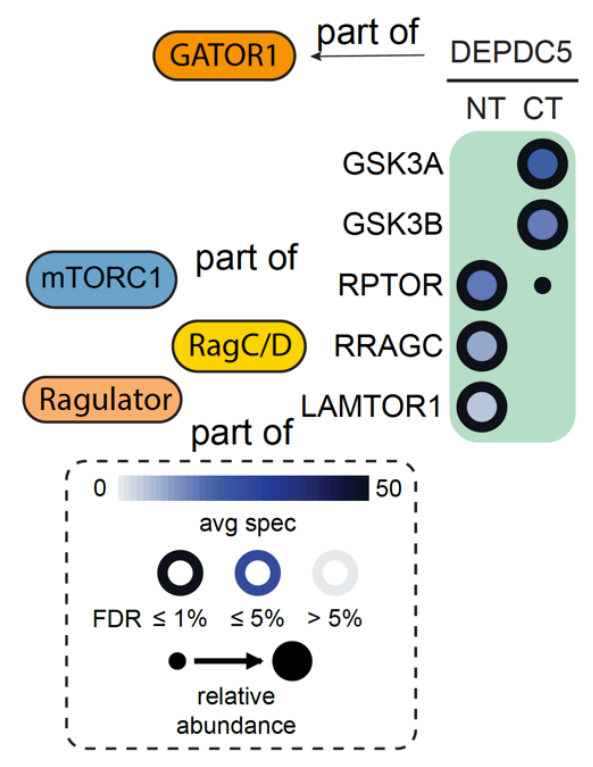

Figure A. 3 Proximity biotinylation (BioID assay) shows interaction between DEPDC5 and GSK3 $\alpha$ and $\boldsymbol{\beta}$. DEPDC5 was tagged with BirA on either N- or C- terminal, which are stably expressed in HEK293 Flp-In T-Rex cells. Bait expression $(1 \mu \mathrm{g} / \mathrm{mL}$ tet.) and biotinylation of proteins ( $50 \mu \mathrm{M}$ biotin) were simultaneously induced. Cells were lysed after 24 $\mathrm{h}$ and biotinylated prey proteins were purified using streptavidin-sepharose. Peptides were released from prey proteins by tryptic digestion and then subjected to mass spectrometry for analysis. High confidence DEPDC5 proximity interactors were determined using SAINT (Teo et al., 2014) and selected preys were visualized with a dot plot using prohits-viz.lunenfeld.ca (Knight et la., 2017). FDR: false discovery rate. Thus, DEPDC5 and GSK3 $\alpha$ and $\beta$ are high interacting partners with high confidence. Hesketh $G$, Gingras AC, unpublished. 


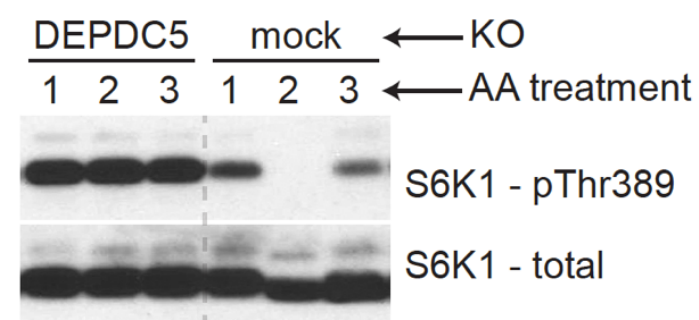

Figure A. 4 DEPDC5 KO hyperactivates mTORC1 activity. HEK293 cells were subjected to CRISPR/Cas9 genome editing to knockout (KO) DEPDC5. DEPDC5 KO cells contains hyperactivated mTORC1 activity, as observed by pS6K1, which is an mTORC1 substrate. Hesketh G, Gingras AC, unpublished. 
References

A., F.-M., \& E., S. (2011). Ras in cancer and developmental diseases. Genes and Cancer, 2(3), 344-358. Retrieved from http://ovidsp.ovid.com/ovidweb.cgi?T=JS\&PAGE=reference\&D=emed10\&NEWS=N\&AN $=2011374475$

A., F. S., \& Sergio, G. (2014). Phagocytosis: receptors, signal integration, and the cytoskeleton. Immunological Reviews, 262(1), 193-215. https://doi.org/10.1111/imr.12212

Abrams, H. D., Rohrschneider, L. R., \& Eisenman, R. N. (1982). Nuclear location of the putative transforming protein of avian myelocytomatosis virus. Cell, 29(2), 427-439. https://doi.org/10.1016/0092-8674(82)90159-3

Almena, M., \& Mérida, I. (2011). Shaping up the membrane: Diacylglycerol coordinates spatial orientation of signaling. Trends in Biochemical Sciences, 36(11), 593-603. https://doi.org/10.1016/j.tibs.2011.06.005

Angliker, N., \& Rüegg, M. A. (2013). In vivo evidence for mTORC2-mediated actin cytoskeleton rearrangement in neurons. BioArchitecture, 3(4), 113-118. https://doi.org/10.4161/bioa.26497

Antonny, B., Burd, C., De Camilli, P., Chen, E., Daumke, O., Faelber, K., ... Schmid, S. (2016). Membrane fission by dynamin: what we know and what we need to know. The EMBO Journal, 35(21), 2270-2284. https://doi.org/10.15252/embj.201694613

Apodaca, G., \& Brown, W. J. (2014). Membrane traffic research: challenges for the next decade. Frontiers in Cell and Developmental Biology, 2. https://doi.org/10.3389/fcell.2014.00052

Araujo, D. M., \& Cotman, C. W. (1992). $\beta$-Amyloid stimulates glial cells in vitro to produce growth factors that accumulate in senile plaques in Alzheimer's disease. Brain Research, 569(1), 141-145. https://doi.org/10.1016/0006-8993(92)90380-R

Avruch, J. (2007). MAP kinase pathways: The first twenty years. Biochimica et Biophysica Acta - Molecular Cell Research, 1773(8), 1150-1160. https://doi.org/10.1016/j.bbamcr.2006.11.006

Azoulay-Alfaguter, I., Elya, R., Avrahami, L., Katz, A., \& Eldar-Finkelman, H. (2015). Combined regulation of mTORC1 and lysosomal acidification by GSK-3 suppresses autophagy and contributes to cancer cell growth. Oncogene, 34(35), 4613-4623. https://doi.org/10.1038/onc.2014.390

Azoulay-Alfaguter, I., Yaffe, Y., Licht-Murava, A., Urbanska, M., Jaworsk, J., Pietrokovski, S., ... Eldar-Finkelman, H. (2011). Distinct molecular regulation of glycogen synthase kinase- 
$3 \alpha$ isozyme controlled by its N-terminal region functional role in CALCIUM/CALPAIN signaling. Journal of Biological Chemistry, 286(15), 13470-13480.

https://doi.org/10.1074/jbc.M110.127969

Bajaj, L., Lotfi, P., Pal, R., Ronza, A. di, Sharma, J., \& Sardiello, M. (2018). Lysosome biogenesis in health and disease. Journal of Neurochemistry.

https://doi.org/10.1111/jnc. 14564

Balderhaar, H. J. k., \& Ungermann, C. (2013). CORVET and HOPS tethering complexes coordinators of endosome and lysosome fusion. Journal of Cell Science, 126(6), 13071316. https://doi.org/10.1242/jcs. 107805

Bar-Peled, L., Chantranupong, L., Cherniack, A. D., Chen, W. W., Ottina, K. A., Grabiner, B. C., ... Sabatini, D. M. (2013). A Tumor Suppressor Complex with GAP Activity for the Rag GTPases That Signal Amino Acid Sufficiency to mTORC1. Science, 340(6136), 11001106. https://doi.org/10.1126/science. 1232044

Bar-Peled, L., Schweitzer, L. D., Zoncu, R., \& Sabatini, D. M. (2012). Ragulator is a GEF for the rag GTPases that signal amino acid levels to mTORC1. Cell, 150(6), 1196-1208. https://doi.org/10.1016/j.cell.2012.07.032

Batlle, E., \& Clevers, H. (2017). Cancer stem cells revisited. Nature Medicine, 23(10), 1124 1134. https://doi.org/10.1038/nm.4409

Baudino, T. A., McKay, C., Pendeville-Samain, H., Nilsson, J. A., Maclean, K. H., White, E. L., ... Cleveland, J. L. (2002). c-Myc is essential for vasculogenesis and angiogenesis during development and tumor progression. Genes and Development, 16(19), 2530-2543. https://doi.org/10.1101/gad.1024602

Bauerfeind, R., Takei, K., \& De Camilli, P. (1997). Amphiphysin I is associated with coated endocytic intermediates and undergoes stimulation-dependent dephosphorylation in nerve terminals. Journal of Biological Chemistry, 272(49), 30984-30992.

https://doi.org/10.1074/jbc.272.49.30984

Bechard, M., \& Dalton, S. (2009). Subcellular Localization of Glycogen Synthase Kinase 3 Controls Embryonic Stem Cell Self-Renewal. Molecular and Cellular Biology, 29(8), 2092-2104. https://doi.org/10.1128/mcb.01405-08

Benzeno, S., \& Diehl, J. A. (2004). C-terminal sequences direct cyclin D1-CRM1 binding. Journal of Biological Chemistry, 279(53), 56061-56066. https://doi.org/10.1074/jbc.M411910200

Berridge, M. J., \& Irvine, R. F. (1984). Inositol trisphosphate, a novel second messenger in cellular signal transduction. Nature, 312(5992), 315-321. https://doi.org/10.1038/312315a0 
Betz, C., \& Hall, M. N. (2013). Where is mTOR and what is it doing there? The Journal of Cell Biology, 203(4), 563-574. https://doi.org/10.1083/jcb.201306041

Beurel, E., Grieco, S. F., \& Jope, R. S. (2015). Glycogen synthase kinase-3 (GSK3): Regulation, actions, and diseases. Pharmacology \& Therapeutics, 148(9), 114-131.

https://doi.org/10.1016/j.pharmthera.2014.11.016

Bhagwat, A. S., \& Vakoc, C. R. (2015). Targeting Transcription Factors in Cancer. Trends in Cancer, 1(1), 53-65. https://doi.org/10.1016/j.trecan.2015.07.001

Bijur, G. N., \& Jope, R. S. (2001). Proapoptotic Stimuli Induce Nuclear Accumulation of Glycogen Synthase Kinase-3ß. Journal of Biological Chemistry, 276(40), 37436-37442. https://doi.org/10.1074/jbc.M105725200

Binder, Z. A., Thorne, A. H., Bakas, S., Wileyto, E. P., Bilello, M., Akbari, H., ... O’Rourke, D. M. (2018). Epidermal Growth Factor Receptor Extracellular Domain Mutations in Glioblastoma Present Opportunities for Clinical Imaging and Therapeutic Development. Cancer Cell, 34(1), 163-177.e7. https://doi.org/10.1016/j.ccell.2018.06.006

Birkenmeier, K., Dröse, S., Wittig, I., Winkelmann, R., Käfer, V., Döring, C., ... Hansmann, M. L. (2016). Hodgkin and Reed-Sternberg cells of classical Hodgkin lymphoma are highly dependent on oxidative phosphorylation. International Journal of Cancer, 138(9), 22312246. https://doi.org/10.1002/ijc.29934

Blackadar, C. B. (2016). Historical review of the causes of cancer. World Journal of Clinical Oncology, 7(1), 54. https://doi.org/10.5306/wjco.v7.i1.54

BOLTE, S., \& CORDELIÈRES, F. P. (2006). A guided tour into subcellular colocalization analysis in light microscopy. Journal of Microscopy, 224(3), 213-232.

https://doi.org/10.1111/j.1365-2818.2006.01706.x

Bone, L. N., Dayam, R. M., Lee, M., Kono, N., Fairn, G. D., Arai, H., ... Antonescu, C. N. (2017). The acyltransferase LYCAT controls specific phosphoinositides and related membrane traffic. Molecular Biology of the Cell, 28(1), 161-172. https://doi.org/10.1091/mbc.e16-09-0668

Borisov, N., Aksamitiene, E., Kiyatkin, A., Legewie, S., Berkhout, J., Maiwald, T., ... Kholodenko, B. N. (2009). Systems-level interactions between insulin-EGF networks amplify mitogenic signaling. Molecular Systems Biology, 5. https://doi.org/10.1038/msb.2009.19

Boroughs, L. K., \& DeBerardinis, R. J. (2015). Metabolic pathways promoting cancer cell survival and growth. Nature Cell Biology, 17(4), 351-359. https://doi.org/10.1038/ncb3124

Boucrot, E., Saffarian, S., Zhang, R., \& Kirchhausen, T. (2010). Roles of AP-2 in clathrinmediated endocytosis. PLoS ONE, 5(5). https://doi.org/10.1371/journal.pone.0010597 
Bozóky, Z., Róna, G., Klement, É., Medzihradszky, K. F., Merényi, G., Vértessy, B. G., \& Friedrich, P. (2011). Calpain-catalyzed proteolysis of human dutpase specifically removes the nuclear localization signal peptide. PLoS ONE, 6(5). https://doi.org/10.1371/journal.pone.0019546

Brabletz, T., Jung, A., Dag, S., Hlubek, F., \& Kirchner, T. (1999). $\beta$-Catenin Regulates the Expression of the Matrix Metalloproteinase-7 in Human Colorectal Cancer. The American Journal of Pathology, 155(4), 1033-1038. https://doi.org/10.1016/S0002-9440(10)65204-2

Brown, E. J., Albers, M. W., Bum Shin, T., Ichikawa, K., Keith, C. T., Lane, W. S., \& Schreiber, S. L. (1994). A mammalian protein targeted by G1-arresting rapamycin-receptor complex. Nature, 369(6483), 756-758. https://doi.org/10.1038/369756a0

Buday, L., \& Tompa, P. (2010). Functional classification of scaffold proteins and related molecules. FEBS Journal, 277(21), 4348-4355. https://doi.org/10.1111/j.17424658.2010.07864.x

C., W., K., G., D., G., X., K., K., J., Y., L., .. Y., L. (2011). Identification of transaldolase as a novel serum biomarker for hepatocellular carcinoma metastasis using xenografted mouse model and clinic samples. Cancer Letters, 313(2), 154-166. Retrieved from http://ovidsp.ovid.com/ovidweb.cgi?T $=\mathrm{JS} \& \mathrm{PAGE}=$ reference $\& \mathrm{D}=\mathrm{emed} 10 \& \mathrm{NEWS}=\mathrm{N} \& A \mathrm{~N}$ $=2011664562$

Cao, Q., Lu, X., \& Feng, Y. J. (2006). Glycogen synthase kinase-3 $\beta$ positively regulates the proliferation of human ovarian cancer cells. Cell Research, 16(7), 671-677. https://doi.org/10.1038/sj.cr.7310078

Carey, K. L., Richards, S. A., Lounsbury, K. M., \& Macara, I. G. (1996). Evidence using a green fluorescent protein-glucocorticoid receptor chimera that the RAN/TC4 GTPase mediates an essential function independent of nuclear protein import. Journal of Cell Biology, 133(5), 985-996. https://doi.org/10.1083/jcb.133.5.985

Cargnello, M., \& Roux, P. P. (2011). Activation and Function of the MAPKs and Their Substrates, the MAPK-Activated Protein Kinases. Microbiology and Molecular Biology Reviews, 75(1), 50-83. https://doi.org/10.1128/mmbr.00031-10

Castellano, B. M., Thelen, A. M., Moldavski, O., Feltes, M., van der Welle, R. E. N., MydockMcGrane, L., ... Zoncu, R. (2017). Lysosomal cholesterol activates mTORC1 via an SLC38A9-Niemann-Pick C1 signaling complex. Science, 355(6331), 1306-1311. https://doi.org/10.1126/science.aag1417

Cautain, B., Hill, R., de Pedro, N., \& Link, W. (2015). Components and regulation of nuclear transport processes. FEBS Journal, 282(3), 445-462. https://doi.org/10.1111/febs.13163 
Chan, C. H., Lee, S. W., Li, C. F., Wang, J., Yang, W. L., Wu, C. Y., ... Lin, H. K. (2010). Deciphering the transcriptional complex critical for RhoA gene expression and cancer metastasis. Nature Cell Biology, 12(5), 457-467. https://doi.org/10.1038/ncb2047

Chantranupong, L., Scaria, S. M., Saxton, R. A., Gygi, M. P., Shen, K., Wyant, G. A., ... Sabatini, D. M. (2016). The CASTOR Proteins Are Arginine Sensors for the mTORC1 Pathway. Cell, 165(1), 153-164. https://doi.org/10.1016/j.cell.2016.02.035

Chantranupong, L., Wolfson, R. L., Orozco, J. M., Saxton, R. A., Scaria, S. M., Bar-Peled, L., ... Sabatini, D. M. (2014). The Sestrins Interact with GATOR2 to Negatively Regulate the Amino-Acid-Sensing Pathway Upstream of mTORC1. Cell Reports, 9(1), 1-8. https://doi.org/10.1016/j.celrep.2014.09.014

Chen, H., Slepnev, V. I., Di Fiore, P. P., \& De Camilli, P. (1999). The interaction of Epsin and Eps15 with the clathrin adaptor AP-2 is inhibited by mitotic phosphorylation and enhanced by stimulation-dependent dephosphorylation in nerve terminals. Journal of Biological Chemistry, 274(6), 3257-3260. https://doi.org/10.1074/jbc.274.6.3257

Chen, H., Liu, H., \& Qing, G. (2018). Targeting oncogenic Myc as a strategy for cancer treatment. Signal Transduction and Targeted Therapy, 3(1), 5. https://doi.org/10.1038/s41392-018-0008-7

Chen, K., Ding, X., Sun, Q., Chen, S., Zhong, Q., \& Fan, W. (2008). Identification of Barkor as a mammalian autophagy-specific factor for Beclin 1 and class III phosphatidylinositol 3kinase. Proceedings of the National Academy of Sciences, 105(49), 19211-19216. https://doi.org/10.1073/pnas.0810452105

Chen, P. H., Bendris, N., Hsiao, Y. J., Reis, C. R., Mettlen, M., Chen, H. Y., ... Schmid, S. L. (2017). Crosstalk between CLCb/Dyn1-Mediated Adaptive Clathrin-Mediated Endocytosis and Epidermal Growth Factor Receptor Signaling Increases Metastasis. Developmental Cell, 40(3), 278-288.e5. https://doi.org/10.1016/j.devcel.2017.01.007

Cho, K. Bin, Cho, M. K., Lee, W. Y., \& Kang, K. W. (2010). Overexpression of c-Myc induces epithelial mesenchymal transition in mammary epithelial cells. Cancer Letters, 293(2), 230 239. https://doi.org/10.1016/j.canlet.2010.01.013

Cho, W. (2001). Membrane Targeting by C1 and C2 Domains. Journal of Biological Chemistry, 276(35), 32407-32410. https://doi.org/10.1074/jbc.R100007200

Choi, J. H., Park, J. B., Bae, S. S., Yun, S., Kim, H. S., Hong, W.-P., ... Suh, P.-G. (2004). Phospholipase C-gammal is a guanine nucleotide exchange factor for dynamin-1 and enhances dynamin-1-dependent epidermal growth factor receptor endocytosis. Journal of Cell Science, 117(Pt 17), 3785-3795. https://doi.org/10.1242/jcs.01220

Choudhury, A., Dominguez, M., Puri, V., Sharma, D. K., Narita, K., Wheatley, C. L., ... Pagano, R. E. (2002). Rab proteins mediate Golgi transport of caveola-internalized 
glycosphingolipids and correct lipid trafficking in Niemann-Pick C cells. Journal of Clinical Investigation, 109(12), 1541-1550. https://doi.org/10.1172/JCI0215420

Chu, B., Liao, Y., Qi, W., Xie, C., Du, X., Wang, J., ... Song, B.-L. (2015). Cholesterol Transport through Lysosome-Peroxisome Membrane Contacts. Cell, 161(2), 291-306. https://doi.org/10.1016/j.cell.2015.02.019

Chung, I., Akita, R., Vandlen, R., Toomre, D., Schlessinger, J., \& Mellman, I. (2010). Spatial control of EGF receptor activation by reversible dimerization on living cells. Nature, 464(7289), 783-787. https://doi.org/10.1038/nature08827

Chung, J., Kuo, C. J., Crabtree, G. R., \& Blenis, J. (1992). Rapamycin-FKBP specifically blocks growth-dependent activation of and signaling by the $70 \mathrm{kd} \mathrm{S6}$ protein kinases. Cell, 69(7), 1227-1236. https://doi.org/10.1016/0092-8674(92)90643-Q

Cianfanelli, V., Fuoco, C., Lorente, M., Salazar, M., Quondamatteo, F., Gherardini, P. F., ... Cecconi, F. (2015). Erratum: Corrigendum: AMBRA1 links autophagy to cell proliferation and tumorigenesis by promoting c-Myc dephosphorylation and degradation. Nature Cell Biology, 17(5), 706-706. https://doi.org/10.1038/ncb3171

Ciardiello, F., \& Tortora, G. (2008). EGFR Antagonists in Cancer Treatment. New England Journal of Medicine, 358(11), 1160-1174. https://doi.org/10.1056/NEJMra0707704

Clapham, D. E. (2007). Calcium Signaling. Cell, 131(6), 1047-1058. https://doi.org/10.1016/j.cell.2007.11.028

Clayton, E. L., Sue, N., Smillie, K. J., O'Leary, T., Bache, N., Cheung, G., ... Cousin, M. a. (2010). Dynamin I phosphorylation by GSK3 controls activity-dependent bulk endocytosis of synaptic vesicles. Nature Neuroscience, 13(7), 845-851. https://doi.org/10.1038/nn.2571

Cocucci, E., Aguet, F., Boulant, S., \& Kirchhausen, T. (2012). The first five seconds in the life of a clathrin-coated pit. Cell, 150(3), 495-507. https://doi.org/10.1016/j.cell.2012.05.047

Cohen, P., \& Frame, S. (2001). The renaissance of GSK3. Nature Reviews Molecular Cell Biology, 2(10), 769-776. https://doi.org/10.1038/35096075

COLE, A., FRAME, S., \& COHEN, P. (2004). Further evidence that the tyrosine phosphorylation of glycogen synthase kinase-3 (GSK3) in mammalian cells is an autophosphorylation event. Biochemical Journal, 377(1), 249-255. https://doi.org/10.1042/bj20031259

Conley-LaComb, M. K., Saliganan, A., Kandagatla, P., Chen, Y. Q., Cher, M. L., \& Chinni, S. R. (2013). PTEN loss mediated Akt activation promotes prostate tumor growth and metastasis via CXCL12/CXCR4 signaling. Molecular Cancer, 12(1). https://doi.org/10.1186/1476-4598-12-85 
Conte, A., \& Sigismund, S. (2016). Chapter Six - The Ubiquitin Network in the Control of EGFR Endocytosis and Signaling. Progress in Molecular Biology and Translational Science, 141, 225-276. https://doi.org/10.1016/bs.pmbts.2016.03.002

Cormier, K. W., \& Woodgett, J. R. (2017). Recent advances in understanding the cellular roles of GSK-3. F1000Research, 6, 167. https://doi.org/10.12688/f1000research.10557.1

Cousin, M. A., \& Robinson, P. J. (2001). The dephosphins: Dephosphorylation by calcineurin triggers synaptic vesicle endocytosis. Trends in Neurosciences, 24(11), 659-665. https://doi.org/10.1016/S0166-2236(00)01930-5

Cremona, O., \& De Camilli, P. (1997). Synaptic vesicle endocytosis. Current Opinion in Neurobiology, 7(3), 323-330. https://doi.org/10.1016/S0959-4388(97)80059-1

Cross, D. A. E., Alessi, D. R., Cohen, P., Andjelkovich, M., \& Hemmings, B. A. (1995). Inhibition of glycogen synthase kinase- 3 by insulin mediated by protein kinase B. Nature, 378(6559), 785-789. https://doi.org/10.1038/378785a0

Dajani, R., Fraser, E., Roe, S. M., Young, N., Good, V., Dale, T. C., \& Pearl, L. H. (2001). Crystal structure of glycogen synthase kinase $3 \beta$ : Structural basis for phosphate-primed substrate specificity and autoinhibition. Cell, 105(6), 721-732. https://doi.org/10.1016/S0092-8674(01)00374-9

Dang, C. V. (2008). The Interplay Between MYC and HIF in the Warburg Effect, 35-53. https://doi.org/10.1007/2789_2008_088

Dang, C. V., O’Donnell, K. A., Zeller, K. I., Nguyen, T., Osthus, R. C., \& Li, F. (2006). The cMyc target gene network. Seminars in Cancer Biology, 16(4), 253-264. https://doi.org/10.1016/j.semcancer.2006.07.014

Dansen, T. B., \& Burgering, B. M. T. (2008). Unravelling the tumor-suppressive functions of FOXO proteins. Trends in Cell Biology, 18(9), 421-429. https://doi.org/10.1016/j.tcb.2008.07.004

Darnell, J. E. (2002). Transcription factors as targets for cancer therapy. Nature Reviews Cancer, 2(10), 740-749. https://doi.org/10.1038/nrc906

De Palma, M., Biziato, D., \& Petrova, T. V. (2017). Microenvironmental regulation of tumour angiogenesis. Nature Reviews. Cancer, 17(8), 457-474. https://doi.org/10.1038/nrc.2017.51

De Strooper, B., Vassar, R., \& Golde, T. (2010). The secretases: Enzymes with therapeutic potential in Alzheimer disease. Nature Reviews Neurology, 6(2), 99-107. https://doi.org/10.1038/nrneurol.2009.218 
DeFronzo, R. A., Ferrannini, E., Groop, L., Henry, R. R., Herman, W. H., Holst, J. J., ... Weiss, R. (2015). Type 2 diabetes mellitus. Nature Reviews Disease Primers, 1(1), 15019. https://doi.org/10.1038/nrdp.2015.19

Delos Santos, R. C., Bautista, S., Lucarelli, S., Bone, L. N., Dayam, R. M., Abousawan, J., ... Antonescu, C. N. (2017). Selective regulation of clathrin-mediated epidermal growth factor receptor signaling and endocytosis by phospholipase $\mathrm{C}$ and calcium. Molecular Biology of the Cell, 28(21), 2802-2818. https://doi.org/10.1091/mbc.e16-12-0871

Dews, M., Fox, J. L., Hultine, S., Sundaram, P., Wang, W., Liu, Y. Y., ... Thomas-Tikhonenko, A. (2010). The Myc-miR-17 92 Axis Blunts TGF Signaling and Production of Multiple TGF -Dependent Antiangiogenic Factors. Cancer Research, 70(20), 8233-8246. https://doi.org/10.1158/0008-5472.CAN-10-2412

Dhillon, A. S., Hagan, S., Rath, O., \& Kolch, W. (2007). MAP kinase signalling pathways in cancer. Oncogene, 26(22), 3279-3290. https://doi.org/10.1038/sj.onc.1210421

Diaz, R., Mayorga, L., \& Stahl, P. (1988). In vitro fusion of endosomes following receptormediated endocytosis. Journal of Biological Chemistry, 263(13), 6093-6100.

Dibble, C. C., \& Cantley, L. C. (2015). Regulation of mTORC1 by PI3K signaling. Trends in Cell Biology, 25(9), 545-555. https://doi.org/10.1016/j.tcb.2015.06.002

Diehl, J. A., Cheng, M., Roussel, M. F., \& Sherr, C. J. (1998). Glycogen synthase kinase-3beta regulates cyclin D1 proteolysis and subcellular localization. Genes \& Development, 12(22), 3499-3511. https://doi.org/10.1101/gad.12.22.3499

Diggins, N. L., \& Webb, D. J. (2017). APPL1 is a multifunctional endosomal signaling adaptor protein. Biochemical Society Transactions, 45(3), 771-779.

https://doi.org/10.1042/bst20160191

Ding, Q., Xia, W., Liu, J. C., Yang, J. Y., Lee, D. F., Xia, J., ... Hung, M. C. (2005). Erk associates with and primes GSK-3 $\beta$ for its inactivation resulting in upregulation of $\beta$ catenin. Molecular Cell, 19(2), 159-170. https://doi.org/10.1016/j.molcel.2005.06.009

Ding, Q., Xia, W., Liu, J.-C., Yang, J.-Y., Lee, D.-F., Xia, J., ... Hung, M.-C. (2005). Erk Associates with and Primes GSK-3 $\beta$ for Its Inactivation Resulting in Upregulation of $\beta$ Catenin. Molecular Cell, 19(2), 159-170. https://doi.org/10.1016/j.molcel.2005.06.009

Dingwall, C., \& Laskey, R. A. (1991). Nuclear targeting sequences - a consensus? Trends in Biochemical Sciences, 16(C), 478-481. https://doi.org/10.1016/0968-0004(91)90184-W

Doherty, G. J., \& McMahon, H. T. (2009). Mechanisms of Endocytosis. Annual Review of Biochemistry, 78(1), 857-902. https://doi.org/10.1146/annurev.biochem.78.081307.110540 
Douglas, H., \& Robert, A. W. (2011). Hallmarks of Cancer: The Next Generation. Cell, 144(1), 646-674. https://doi.org/10.1016/j.cell.2011.02.013

Du, Z., \& Lovly, C. M. (2018). Mechanisms of receptor tyrosine kinase activation in cancer. Molecular Cancer, 17(1). https://doi.org/10.1186/s12943-018-0782-4

É., A.-W., C.R., D., T., Y., F.Z., Z., M., Y., G., K., .. V., G. (2017). Nuclear mTOR acts as a transcriptional integrator of the androgen signaling pathway in prostate cancer. Genes and Development, 31(12), 1228-1242. https://doi.org/10.1101/gad.299958.117

E., G., \& T.E., M. (2009). The Akt kinases: Isoform specificity in metabolism and cancer. Cell Cycle, 8(16), 2502-2508. Retrieved from http://www.landesbioscience.com/journals/cc/article/GonzalezCC816.pdf\%5Cnhttp://ovidsp.ovid.com/ovidweb.cgi?T=JS\&PAGE=reference\&D $=$ emed9\&NE $\mathrm{WS}=\mathrm{N} \& \mathrm{AN}=2009457281$

Eddy, R. J., Weidmann, M. D., Sharma, V. P., \& Condeelis, J. S. (2017). Tumor Cell Invadopodia: Invasive Protrusions that Orchestrate Metastasis. Trends in Cell Biology, 27(8), 595-607. https://doi.org/10.1016/j.tcb.2017.03.003

Efeyan, A., Zoncu, R., \& Sabatini, D. M. (2012). Amino acids and mTORC1: from lysosomes to disease. Trends in Molecular Medicine, 18(9), 524-533.

https://doi.org/10.1016/j.molmed.2012.05.007

Eichel, K., Jullié, D., \& Von Zastrow, M. (2016). $\beta$-Arrestin drives MAP kinase signalling from clathrin-coated structures after GPCR dissociation. Nature Cell Biology, 18(3), 303-310. https://doi.org/10.1038/ncb3307

Eichel, K., Jullié, D., Barsi-Rhyne, B., Latorraca, N. R., Masureel, M., Sibarita, J. B., ... Von Zastrow, M. (2018). Catalytic activation of $\beta$-Arrestin by GPCRs. Nature, 557(7705), 381386. https://doi.org/10.1038/s41586-018-0079-1

Eldar-Finkelman, H., Schreyer, S. A., Shinohara, M. M., LeBoeuf, R. C., \& Krebs, E. G. (1999). Increased glycogen synthase kinase- 3 activity in diabetes- and obesity-prone C57BL/6J mice. Diabetes, 48(8), 1662-1666. https://doi.org/10.2337/diabetes.48.8.1662

Elenko, E., Fischer, T., Niesman, I., Harding, T., McQuistan, T., Von Zastrow, M., \& Farquhar, M. G. (2003). Spatial regulation of Galphai protein signaling in clathrin-coated membrane microdomains containing GAIP. Molecular Pharmacology, 64(1), 11-20. https://doi.org/10.1124/mol.64.1.11

EMBI, N., RYLATT, D. B., \& COHEN, P. (2005). Glycogen Synthase Kinase-3 from Rabbit Skeletal Muscle. European Journal of Biochemistry, 107(2), 519-527. https://doi.org/10.1111/j.1432-1033.1980.tb06059.x 
ENG, C. P., SEHGAL, S. N., \& VEZINA, C. (1984). Activity of rapamycin (AY-22,989) against transplanted tumors. The Journal of Antibiotics, 37(10), 1231-1237.

https://doi.org/10.7164/antibiotics.37.1231

Epstein, T., Xu, L., Gillies, R. J., \& Gatenby, R. A. (2014). Separation of metabolic supply and demand: aerobic glycolysis as a normal physiological response to fluctuating energetic demands in the membrane. Cancer \& Metabolism, 2(1), 7. https://doi.org/10.1186/20493002-2-7

Fagerlund, R., Kinnunen, L., Köhler, M., Julkunen, I., \& Melén, K. (2005). NF-кB Is Transported into the Nucleus by Importin $\alpha 3$ and Importin $\alpha 4$. Journal of Biological Chemistry, 280(16), 15942-15951. https://doi.org/10.1074/jbc.M500814200

Fairn, G. D., \& Grinstein, S. (2012). How nascent phagosomes mature to become phagolysosomes. Trends in Immunology, 33(8), 397-405.

https://doi.org/10.1016/j.it.2012.03.003

Fekri, F., Delos Santos, R. C., Karshafian, R., \& Antonescu, C. N. (2016). Ultrasound microbubble treatment enhances clathrin-mediated endocytosis and fluid-phase uptake through distinct mechanisms. PLoS ONE, 11(6).

https://doi.org/10.1371/journal.pone.0156754

Ferreira, A. P. A., \& Boucrot, E. (2018). Mechanisms of Carrier Formation during ClathrinIndependent Endocytosis. Trends in Cell Biology, 28(3), 188-200.

https://doi.org/10.1016/j.tcb.2017.11.004

Fletcher, J. A., Tsao, M.-S., Mc Henry, K. T., True, L. D., Okamoto, A., Baselga, J., ... Gao, Q. (2010). The landscape of somatic copy-number alteration across human cancers. Nature, 463(7283), 899-905. https://doi.org/10.1038/nature08822

Flinn, R. J., Goswami, S., Parker, P. J., Backer, J. M., \& Yan, Y. (2010). The Late Endosome is Essential for mTORC1 Signaling. Molecular Biology of the Cell, 21(5), 833-841. https://doi.org/10.1091/mbc.e09-09-0756

Flinn, R. J., Yan, Y., Goswami, S., Parker, P. J., \& Backer, J. M. (2010). The Late Endosome is Essential for mTORC1 Signaling. Molecular Biology of the Cell, 21(5), 833-841. https://doi.org/10.1091/mbc.e09-09-0756

Forrester, S. J., Kawai, T., Elliott, K. J., Brien, S. O., Harris, R. C., \& Eguchi, S. (2016). EGFR transactivation: mechanisms, pathophysiology and potential therapies in cardiovascular system. Annu Rev Pharmacol Toxicol, 56, 627-653. https://doi.org/10.1146/annurevpharmtox-070115-095427.EGFR

Frame, S., Cohen, P., \& Biondi, R. M. (2001). A common phosphate binding site explains the unique substrate specificity of GSK3 and its inactivation by phosphorylation. Molecular Cell, 7(6), 1321-1327. https://doi.org/10.1016/S1097-2765(01)00253-2 
Franca-Koh, J., Yeo, M., Fraser, E., Young, N., \& Dale, T. C. (2002). The regulation of glycogen synthase kinase-3 nuclear export by Frat/GBP. Journal of Biological Chemistry, 277(46), 43844-43848. https://doi.org/10.1074/jbc.M207265200

Franca-Koh, J., Yeo, M., Fraser, E., Young, N., \& Dale, T. C. (2002). The regulation of glycogen synthase kinase-3 nuclear export by Frat/GBP. Journal of Biological Chemistry, 277(46), 43844-43848. https://doi.org/10.1074/jbc.M207265200

Franovic, A., Gunaratnam, L., Smith, K., Robert, I., Patten, D., \& Lee, S. (2007). Translational up-regulation of the EGFR by tumor hypoxia provides a nonmutational explanation for its overexpression in human cancer. Proceedings of the National Academy of Sciences, 104(32), 13092-13097. https://doi.org/10.1073/pnas.0702387104

Freed, D. M., Bessman, N. J., Kiyatkin, A., Salazar-Cavazos, E., Byrne, P. O., Moore, J. O., ... Lemmon, M. A. (2017). EGFR Ligands Differentially Stabilize Receptor Dimers to Specify Signaling Kinetics. Cell, 171(3), 683-695.e18. https://doi.org/10.1016/j.cell.2017.09.017

Frias, M. A., Thoreen, C. C., Jaffe, J. D., Schroder, W., Sculley, T., Carr, S. A., \& Sabatini, D. M. (2006). mSin1 Is Necessary for Akt/PKB Phosphorylation, and Its Isoforms Define Three Distinct mTORC2s. Current Biology, 16(18), 1865-1870. https://doi.org/10.1016/j.cub.2006.08.001

Fruman, D. A., Chiu, H., Hopkins, B. D., Bagrodia, S., Cantley, L. C., \& Abraham, R. T. (2017). The PI3K Pathway in Human Disease. Cell, 170(4), 605-635. https://doi.org/10.1016/j.cell.2017.07.029

Fulda, S., Gorman, A. M., Hori, O., \& Samali, A. (2010). Cellular stress responses: Cell survival and cell death. International Journal of Cell Biology. https://doi.org/10.1155/2010/214074

Furnari, F. B., Fenton, T., Bachoo, R. M., Mukasa, A., Stommel, J. M., Stegh, A., ... Cavenee, W. K. (2007). Malignant astrocytic glioma: Genetics, biology, and paths to treatment. Genes and Development, 21(21), 2683-2710. https://doi.org/10.1101/gad.1596707

Garay, C., Judge, G., Lucarelli, S., Bautista, S., Pandey, R., Singh, T., \& Antonescu, C. N. (2015). Epidermal growth factor-stimulated Akt phosphorylation requires clathrin or ErbB2 but not receptor endocytosis. Molecular Biology of the Cell, 26(19), 3504-3519. https://doi.org/10.1091/mbc.E14-09-1412

Garlanda, C., Dinarello, C. A., \& Mantovani, A. (2013). The Interleukin-1 Family: Back to the Future. Immunity, 39(6), 1003-1018. https://doi.org/10.1016/j.immuni.2013.11.010

Gharwan, H., \& Groninger, H. (2016). Kinase inhibitors and monoclonal antibodies in oncology: clinical implications. Nature Reviews Clinical Oncology, 13(4), 209-227. https://doi.org/10.1038/nrclinonc.2015.213 
Ghosh, K., De, S., Das, S., Mukherjee, S., \& Sengupta Bandyopadhyay, S. (2016). Withaferin A Induces ROS-Mediated Paraptosis in Human Breast Cancer Cell-Lines MCF-7 and MDAMB-231. PLOS ONE, 11(12), e0168488. https://doi.org/10.1371/journal.pone.0168488

Glick, D., Barth, S., \& Macleod, K. F. (2010). Autophagy: Cellular and molecular mechanisms. Journal of Pathology, 221(1), 3-12. https://doi.org/10.1002/path.2697

Goc, A., Al-Husein, B. A.-H., Katsanevas, K., Steinbach, A., Lou, U., Sabbineni, H., ... Somanath, P. R. (2014). Targeting Src-mediated Tyr216 phosphorylation and activation of GSK-3 in prostate cancer cells inhibit prostate cancer progression in vitro and in vivo. Oncotarget, 5(3). https://doi.org/10.18632/oncotarget.1770

Goh, L. K., \& Sorkin, A. (2013). Endocytosis of receptor tyrosine kinases. Cold Spring Harbor Perspectives in Medicine, 3(4). https://doi.org/10.1101/cshperspect.a017459

Goncalves, M. D., Hopkins, B. D., \& Cantley, L. C. (2018). Phosphatidylinositol 3-Kinase, Growth Disorders, and Cancer. New England Journal of Medicine, 379(21), 2052-2062. https://doi.org/10.1056/nejmra1704560

Goñi-Oliver, P., Lucas, J. J., Avila, J., \& Hernández, F. (2007). N-terminal cleavage of GSK-3 by calpain: A new form of GSK-3 regulation. Journal of Biological Chemistry, 282(31), 22406-22413. https://doi.org/10.1074/jbc.M702793200

Gorvel, J. P., Chavrier, P., Zerial, M., \& Gruenberg, J. (1991). Rab5 Controls Early Endosome Fusion in Vitro. Cell, 64(5), 915-925. https://doi.org/10.1016/0092-8674(91)90316-Q

Goueli, B. S., \& Janknecht, R. (2003). Upregulation of the Catalytic Telomerase Subunit by the Transcription Factor ER81 and Oncogenic HER2/Neu, Ras, or Raf. Molecular and Cellular Biology, 24(1), 25-35. https://doi.org/10.1128/mcb.24.1.25-35.2004

Gowans, G. J., Hawley, S. A., Ross, F. A., \& Hardie, D. G. (2013). AMP is a true physiological regulator of amp-activated protein kinase by both allosteric activation and enhancing net phosphorylation. Cell Metabolism, 18(4), 556-566.

https://doi.org/10.1016/j.cmet.2013.08.019

Grandal, M. V., Zandi, R., Pedersen, M. W., Willumsen, B. M., van Deurs, B., \& Poulsen, H. S. (2007). EGFRvIII escapes down-regulation due to impaired internalization and sorting to lysosomes. Carcinogenesis, 28(7), 1408-1417. https://doi.org/10.1093/carcin/bgm058

Gregory, M. A., Qi, Y., \& Hann, S. R. (2003). Phosphorylation by Glycogen Synthase Kinase-3 Controls c-Myc Proteolysis and Subnuclear Localization. Journal of Biological Chemistry, 278(51), 51606-51612. https://doi.org/10.1074/jbc.M310722200

Gregory, M. A., Qi, Y., \& Hann, S. R. (2003). Phosphorylation by Glycogen Synthase Kinase-3 Controls c-Myc Proteolysis and Subnuclear Localization. Journal of Biological Chemistry, 278(51), 51606-51612. https://doi.org/10.1074/jbc.M310722200 
Grossman, E., Medalia, O., \& Zwerger, M. (2012). Functional Architecture of the Nuclear Pore Complex. Annual Review of Biophysics, 41(1), 557-584. https://doi.org/10.1146/annurevbiophys-050511-102328

Gu, X., Orozco, J. M., Saxton, R. A., Condon, K. J., Liu, G. Y., Krawczyk, P. A., ... Sabatini, D. M. (2017). SAMTOR is an S-adenosylmethionine sensor for the mTORC1 pathway. Science, 358(6364), 813-818. https://doi.org/10.1126/science.aao3265

Gwinn, D. M., Shackelford, D. B., Egan, D. F., Mihaylova, M. M., Mery, A., Vasquez, D. S., ... Shaw, R. J. (2008). AMPK Phosphorylation of Raptor Mediates a Metabolic Checkpoint. Molecular Cell, 30(2), 214-226. https://doi.org/10.1016/j.molcel.2008.03.003

H., W., H., N., C., W., Y., L., K., W., J., Z., .. S., Y. (2016). The autophagy-lysosomal system in subarachnoid haemorrhage. Journal of Cellular and Molecular Medicine, 20(9), 17701778. https://doi.org/10.1111/jcmm.12855

Haar, E. Vander, Lee, S., Bandhakavi, S., Griffin, T. J., \& Kim, D.-H. (2007). Insulin signalling to mTOR mediated by the Akt/PKB substrate PRAS40. Nature Cell Biology, 9(3), 316-323. https://doi.org/10.1038/ncb1547

Han, W., Zhang, T., Yu, H., Foulke, J. G., \& Tang, C. K. (2006). Hypophosphorylation of residue Y1045 leads to defective downregulation of EGFRvIII. Cancer Biology and Therapy, 5(10), 1361-1368. https://doi.org/10.4161/cbt.5.10.3226

Hanahan, D., \& Weinberg, R. A. (2000). The Hallmarks of Cancer. Cell, 100(1), 57-70. https://doi.org/10.1016/S0092-8674(00)81683-9

Hann, S. R., Abrams, H. D., Rohrschneider, L. R., \& Eisenman, R. N. (1983). Proteins encoded by v-myc and c-Myc oncogenes: Identification and localization in acute leukemia virus transformants and bursal lymphoma cell lines. Cell, 34(3), 789-798. https://doi.org/10.1016/0092-8674(83)90535-4

Hanover, J. A., Krause, M. W., \& Love, D. C. (2010). The hexosamine signaling pathway: OGlcNAc cycling in feast or famine. Biochimica et Biophysica Acta - General Subjects, 1800(2), 80-95. https://doi.org/10.1016/j.bbagen.2009.07.017

Hao, N., Budnik, B. A., Gunawardena, J., \& O’Shea, E. K. (2013). Tunable signal processing through modular control of transcription factor translocation. Science, 339(6118), 460-464. https://doi.org/10.1126/science.1227299

Hara, K., Maruki, Y., Long, X., Yoshino, K. ichi, Oshiro, N., Hidayat, S., ... Yonezawa, K. (2002). Raptor, a binding partner of target of rapamycin (TOR), mediates TOR action. Cell, 110(2), 177-189. https://doi.org/10.1016/S0092-8674(02)00833-4 
Harayama, T., \& Riezman, H. (2018). Understanding the diversity of membrane lipid composition. Nature Reviews Molecular Cell Biology, 19(5), 281-296. https://doi.org/10.1038/nrm.2017.138

Harburger, D. S., \& Calderwood, D. A. (2009). Integrin signalling at a glance. Journal of Cell Science, 122(2), 159-163. https://doi.org/10.1242/jcs.018093

Harder, J., Franchi, L., Muñoz-Planillo, R., Park, J.-H., Reimer, T., \& Núñez, G. (2009). Activation of the Nlrp3 inflammasome by Streptococcus pyogenes requires streptolysin $\mathrm{O}$ and NF-kappa B activation but proceeds independently of TLR signaling and P2X7 receptor. Journal of Immunology (Baltimore, Md. : 1950), 183(9), 5823-5829. https://doi.org/10.4049/jimmunol.0900444

Hardie, D. G., Carling, D., \& Gamblin, S. J. (2011). AMP-activated protein kinase: Also regulated by ADP? Trends in Biochemical Sciences, 36(9), 470-477. https://doi.org/10.1016/j.tibs.2011.06.004

Harskamp, L. R., Gansevoort, R. T., Van Goor, H., \& Meijer, E. (2016). The epidermal growth factor receptor pathway in chronic kidney diseases. Nature Reviews Nephrology, 12(8), 496-506. https://doi.org/10.1038/nrneph.2016.91

Hassanpour, S. H., \& Dehghani, M. (2017). Review of cancer from perspective of molecular. Journal of Cancer Research and Practice, 4(4), 127-129. https://doi.org/10.1016/j.jcrpr.2017.07.001

He, T. C., Sparks, A. B., Rago, C., Hermeking, H., Zawel, L., Da Costa, L. T., ... Kinzler, K. W. (1998). Identification of c-Myc as a target of the APC pathway. Science, 281(5382), 15091512. https://doi.org/10.1126/science.281.5382.1509

Heiden MGV, Cantley LC, \& Thompson CB. (2010). Understanding the Warburg Effect: The Metabolic Requirements of Cell Proliferation. Science, 324(5930), 1029-1033. https://doi.org/10.1126/science.1160809.Understanding

Hergovich, A., Lisztwan, J., Thoma, C. R., Wirbelauer, C., Barry, R. E., \& Krek, W. (2006). Priming-Dependent Phosphorylation and Regulation of the Tumor Suppressor pVHL by Glycogen Synthase Kinase 3. Molecular and Cellular Biology, 26(15), 5784-5796. https://doi.org/10.1128/mcb.00232-06

Hernandez, F., Lucas, J. J., \& Avila, J. (2012). GSK3 and tau: Two convergence points in Alzheimer's disease. Advances in Alzheimer's Disease, 3, 141-144. https://doi.org/10.3233/978-1-61499-154-0-141

Herzig, S., \& Shaw, R. J. (2018). AMPK: Guardian of metabolism and mitochondrial homeostasis. Nature Reviews Molecular Cell Biology, 19(2), 121-135. https://doi.org/10.1038/nrm.2017.95 
Hilger, D., Masureel, M., \& Kobilka, B. K. (2018). Structure and dynamics of GPCR signaling complexes. Nature Structural and Molecular Biology, 25(1), 4-12.

https://doi.org/10.1038/s41594-017-0011-7

Hirsch, F. R., Jänne, P. A., Eberhardt, W. E., Cappuzzo, F., Thatcher, N., Pirker, R., ... Mok, T. S. (2013). Epidermal growth factor receptor inhibition in lung cancer: Status 2012. Journal of Thoracic Oncology, 8(3), 373-384. https://doi.org/10.1097/JTO.0b013e31827ed0ff

Højlund, K., Birk, J. B., Klein, D. K., Levin, K., Rose, A. J., Hansen, B. F., ... Wojtaszewski, J. F. P. (2009). Dysregulation of glycogen synthase COOH- and NH2-terminal phosphorylation by insulin in obesity and type 2 diabetes mellitus. Journal of Clinical Endocrinology and Metabolism, 94(11), 4547-4556. https://doi.org/10.1210/jc.2009-0897

Højlund, K., Stæhr, P., Hansen, B. F., Green, K. A., Hardie, D. G., Richter, E. A., ... Wojtaszewski, J. F. P. (2003). Increased phosphorylation of skeletal muscle glycogen synthase at NH2-terminal sites during physiological hyperinsulinemia in type 2 diabetes. Diabetes, 52(6), 1393-1402. https://doi.org/10.2337/diabetes.52.6.1393

Holgado-Madruga, M., Emlet, D. R., Moscatello, D. K., Godwin, A. K., \& Wong, A. J. (1996). A Grb2-associated docking protein in EGF- and insulin-receptor signalling. Nature, 379(6565), 560-564. https://doi.org/10.1038/379560a0

Holz, M. K., Ballif, B. A., Gygi, S. P., \& Blenis, J. (2005). mTOR and S6K1 mediate assembly of the translation preinitiation complex through dynamic protein interchange and ordered phosphorylation events. Cell, 123(4), 569-580. https://doi.org/10.1016/j.cell.2005.10.024

Honegger, A. M., Kris, R. M., Ullrich, A., \& Schlessinger, J. (2006). Evidence that autophosphorylation of solubilized receptors for epidermal growth factor is mediated by intermolecular cross-phosphorylation. Proceedings of the National Academy of Sciences, 86(3), 925-929. https://doi.org/10.1073/pnas.86.3.925

Hoshi, M., Takashima, A., Noguchi, K., Murayama, M., Sato, M., Kondo, S., ... Imahori, K. (1996). Regulation of mitochondrial pyruvate dehydrogenase activity by tau protein kinase I/glycogen synthase kinase 3 beta in brain. Proceedings of the National Academy of Sciences, 93(7), 2719-2723. https://doi.org/10.1073/pnas.93.7.2719

Hua, R., Cheng, D., Coyaud, É., Freeman, S., Di Pietro, E., Wang, Y., ... Kim, P. K. (2017). VAPs and ACBD5 tether peroxisomes to the ER for peroxisome maintenance and lipid homeostasis. Journal of Cell Biology, 216(2), 367-377. https://doi.org/10.1083/jcb.201608128

Hughes, K., Nikolakaki, E., Plyte, S. E., Totty, N. F., \& Woodgett, J. R. (1993). Modulation of the glycogen synthase kinase-3 family by tyrosine phosphorylation. The EMBO Journal, 12(2), 803-808. https://doi.org/10.1002/j.1460-2075.1993.tb05715.x 
Hulea, L., Gravel, S.-P., Morita, M., Cargnello, M., Uchenunu, O., Im, Y. K., ... Topisirovic, I. (2018). Translational and HIF-1 $\alpha$-Dependent Metabolic Reprogramming Underpin Metabolic Plasticity and Responses to Kinase Inhibitors and Biguanides. Cell Metabolism, 28(6), 817-832.e8. https://doi.org/10.1016/j.cmet.2018.09.001

Hwang, B., Lee, J. H., \& Bang, D. (2018). Single-cell RNA sequencing technologies and bioinformatics pipelines. Experimental \& Molecular Medicine, 50(8), 96. https://doi.org/10.1038/s12276-018-0071-8

Ikeda, S., Kishida, S., Yamamoto, H., Murai, H., Koyama, S., \& Kikuchi, A. (1998). Axin, a negative regulator of the Wnt signaling pathway, forms a complex with GSK-3 $\beta$ and $\beta$ catenin and promotes GSK-3 $\beta$-dependent phosphorylation of $\beta$-catenin. EMBO Journal, 17(5), 1371-1384. https://doi.org/10.1093/emboj/17.5.1371

Inoki, K., Kim, J., \& Guan, K.-L. (2012). AMPK and mTOR in Cellular Energy Homeostasis and Drug Targets. Annual Review of Pharmacology and Toxicology, 52(1), 381-400. https://doi.org/10.1146/annurev-pharmtox-010611-134537

Inoki, K., Li, Y., Xu, T., \& Guan, K. L. (2003). Rheb GTpase is a direct target of TSC2 GAP activity and regulates mTOR signaling. Genes and Development, 17(15), 1829-1834. https://doi.org/10.1101/gad.1110003

Inoki, K., Ouyang, H., Zhu, T., Lindvall, C., Wang, Y., Zhang, X., ... Guan, K.-L. (2006). TSC2 Integrates Wnt and Energy Signals via a Coordinated Phosphorylation by AMPK and GSK3 to Regulate Cell Growth. Cell, 126(5), 955-968. https://doi.org/10.1016/j.cell.2006.06.055

Inoki, K., Zhu, T., \& Guan, K. L. (2003). TSC2 Mediates Cellular Energy Response to Control Cell Growth and Survival. Cell, 115(5), 577-590. https://doi.org/10.1016/S00928674(03)00929-2

Itkonen, H. M., Minner, S., Guldvik, I. J., Sandmann, M. J., Tsourlakis, M. C., Berge, V., ... Mills, I. G. (2013). O-GlcNAc transferase integrates metabolic pathways to regulate the stability of c-Myc in human prostate cancer cells. Cancer Research, 73(16), 5277-5287. https://doi.org/10.1158/0008-5472.CAN-13-0549

J., K., M., K., B., V., \& K.L., G. (2011). AMPK and mTOR regulate autophagy through direct phosphorylation of Ulk1. Nature Cell Biology, 13(2), 132-141. https://doi.org/10.1038/ncb2152

Jaber, N., Dou, Z., Chen, J.-S., Catanzaro, J., Jiang, Y.-P., Ballou, L. M., ... Zong, W.-X. (2012). Class III PI3K Vps34 plays an essential role in autophagy and in heart and liver function. Proceedings of the National Academy of Sciences, 109(6), 2003-2008. https://doi.org/10.1073/pnas.1112848109 
Jacinto, E., Facchinetti, V., Liu, D., Soto, N., Wei, S., Jung, S. Y., ... Su, B. (2006). SIN1/MIP1 Maintains rictor-mTOR Complex Integrity and Regulates Akt Phosphorylation and Substrate Specificity. Cell, 127(1), 125-137. https://doi.org/10.1016/j.cell.2006.08.033

Jacinto, E., Loewith, R., Schmidt, A., Lin, S., Rüegg, M. A., Hall, A., \& Hall, M. N. (2004). Mammalian TOR complex 2 controls the actin cytoskeleton and is rapamycin insensitive. Nature Cell Biology, 6(11), 1122-1128. https://doi.org/10.1038/ncb1183

Jeggo, P. A., Pearl, L. H., \& Carr, A. M. (2016). DNA repair, genome stability and cancer: A historical perspective. Nature Reviews Cancer, 16(1), 35-42. https://doi.org/10.1038/nrc.2015.4

Jewell, J. L., Kim, Y. C., Russell, R. C., Yu, F.-X., Park, H. W., Plouffe, S. W., .. Guan, K.-L. (2015). Differential regulation of mTORC1 by leucine and glutamine. Science, 347(6218), 194-198. https://doi.org/10.1126/science. 1259472

Joerger, A. C., \& Fersht, A. R. (2016). The p53 Pathway: Origins, Inactivation in Cancer, and Emerging Therapeutic Approaches. Annual Review of Biochemistry, 85(1), 375-404. https://doi.org/10.1146/annurev-biochem-060815-014710

Johnson, A. B., \& Lewis, A. (2002). Extracellular Control of Cell Division, Cell Growth, and Apoptosis. Molecular Biology of the Cell. Garland Science. https://doi.org/10.1046/j.13652850.1998.00130.x

JOHNSON, M. (2006). Molecular mechanisms of $\beta 2$-adrenergic receptor function, response, and regulation. Journal of Allergy and Clinical Immunology, 117(1), 18-24. https://doi.org/10.1016/j.jaci.2005.11.012

Jope, R. S., \& Johnson, G. V. W. (2004). The glamour and gloom of glycogen synthase kinase-3. Trends in Biochemical Sciences, 29(2), 95-102. https://doi.org/10.1016/j.tibs.2003.12.004

Jovic, M., Sharma, M., Rahajeng, J., \& Caplan, S. (2010). The early endosome: A busy sorting station for proteins at the crossroads. Histology and Histopathology, 25(1), 99-112. https://doi.org/10.1016/j.bbi.2008.05.010

Joyner, M. J., \& Casey, D. P. (2015). Regulation of increased blood flow (hyperemia) to muscles during exercise: a hierarchy of competing physiological needs. Physiological Reviews, 95(2), 549-601. https://doi.org/10.1152/physrev.00035.2013

Jung, C. H., Jun, C. B., Ro, S.-H., Kim, Y.-M., Otto, N. M., Cao, J., ... Kim, D.-H. (2009). ULK-Atg13-FIP200 complexes mediate mTOR signaling to the autophagy machinery. Molecular Biology of the Cell, 20(7), 1992-2003. https://doi.org/10.1091/mbc.e08-12-1249

Jung, J., Genau, H. M., \& Behrends, C. (2015). Amino Acid-Dependent mTORC1 Regulation by the Lysosomal Membrane Protein SLC38A9. Molecular and Cellular Biology, 35(14), 2479-2494. https://doi.org/10.1128/mcb.00125-15 
Junttila, M. R., \& Evan, G. I. (2009). P53 a Jack of all trades but master of none. Nature Reviews Cancer, 9(11), 821-829. https://doi.org/10.1038/nrc2728

K., D., J.L., Y., S., M., P., R., A.I., L., A.L., S., .. B.D., M. (2010). Activation of a metabolic gene regulatory network downstream of mTOR complex 1. Molecular Cell, 39(2), 171-183. Retrieved from http://ovidsp.ovid.com/ovidweb.cgi?T $=\mathrm{JS} \& \mathrm{PAGE}=$ reference $\& \mathrm{D}=\mathrm{emed}$ \& $\mathrm{NEWS}=\mathrm{N} \& \mathrm{AN}=$ 2010435936

Ka, M., Condorelli, G., Woodgett, J. R., \& Kim, W.-Y. (2014). mTOR regulates brain morphogenesis by mediating GSK3 signaling. Development, 141(21), 4076-4086. https://doi.org/10.1242/dev.108282

Kabachinski, G., \& Schwartz, T. U. (2015). The nuclear pore complex - structure and function at a glance. Journal of Cell Science, 128(3), 423-429. https://doi.org/10.1242/jcs.083246

Kabeya, Y., Mizushima, N., Ueno, T., Yamamoto, A., Kirisako, T., Noda, T., ... Yoshimori, T. (2000). LC3, a mammalian homologue of yeast Apg8p, is localized in autophagosome membranes after processing. The EMBO Journal, 19(21), 5720-5728. https://doi.org/10.1093/emboj/19.21.5720

Kadamur, G., \& Ross, E. M. (2013). Mammalian Phospholipase C. Annual Review of Physiology, 75(1), 127-154. https://doi.org/10.1146/annurev-physiol-030212-183750

Kaidanovich-Beilin, O., \& Woodgett, J. R. (2011). GSK-3: Functional Insights from Cell Biology and Animal Models. Frontiers in Molecular Neuroscience, 4. https://doi.org/10.3389/fnmol.2011.00040

Kajita, M., McClinic, K. N., \& Wade, P. A. (2004). Aberrant Expression of the Transcription Factors Snail and Slug Alters the Response to Genotoxic Stress. Molecular and Cellular Biology, 24(17), 7559-7566. https://doi.org/10.1128/mcb.24.17.7559-7566.2004

Kaksonen, M., \& Roux, A. (2018). Mechanisms of clathrin-mediated endocytosis. Nature Reviews Molecular Cell Biology, 19(5), 313-326. https://doi.org/10.1038/nrm.2017.132

Kalimutho, M., Parsons, K., Mittal, D., López, J. A., Srihari, S., \& Khanna, K. K. (2015). Targeted Therapies for Triple-Negative Breast Cancer: Combating a Stubborn Disease. Trends in Pharmacological Sciences, 36(12), 822-846. https://doi.org/10.1016/j.tips.2015.08.009

Kalkat, M., De Melo, J., Hickman, K. A., Lourenco, C., Redel, C., Resetca, D., ... Penn, L. Z. (2017). MYC deregulation in primary human cancers. Genes, 8(6), 2-30. https://doi.org/10.3390/genes8060151

Kalluri, R., \& Weinberg, R. A. (2009). The basics of epithelial-mesenchymal transition. Journal of Clinical Investigation, 119(6), 1420-1428. https://doi.org/10.1172/JCI39104 
Katritch, V., Cherezov, V., \& Stevens, R. C. (2013). Structure-Function of the G ProteinCoupled Receptor Superfamily. Annual Review of Pharmacology and Toxicology, 53(1), 531-556. https://doi.org/10.1146/annurev-pharmtox-032112-135923

Katsarou, A., Gudbjörnsdottir, S., Rawshani, A., Dabelea, D., Bonifacio, E., Anderson, B. J., ... Lernmark, Å. (2017). Type 1 diabetes mellitus. Nature Reviews Disease Primers, 3(1), 17016. https://doi.org/10.1038/nrdp.2017.16

Kau, T. R., Way, J. C., \& Silver, P. A. (2004). Nuclear transport and cancer: From mechanism to intervention. Nature Reviews Cancer, 4(2), 106-117. https://doi.org/10.1038/nrc1274

Kaushik, S., Massey, A. C., \& Cuervo, A. M. (2006). Lysosome membrane lipid microdomains: novel regulators of chaperone-mediated autophagy. The EMBO Journal, 25(17), 39213933. https://doi.org/10.1038/sj.emboj.7601283

Kawasaki, T., \& Kawai, T. (2014). Toll-like receptor signaling pathways. Frontiers in Immunology, 5(5625), 461. https://doi.org/10.3389/fimmu.2014.00461

Kazerounian, S., Yee, K. O., \& Lawler, J. (2008). Thrombospondins in cancer. Cell Mol Life Sci., 65(5), 700-712. https://doi.org/10.1016/j.pestbp.2011.02.012.Investigations

Kenneth, W. D., Malgorzata, M. K., \& John, H. M. (2011). A practical guide to evaluating colocalization in biological microscopy. American Journal of Physiology-Cell Physiology, 300, C723-C742. https://doi.org/10.1152/ajpcell.00462.2010.

Kidd, M. (1963). Paired helical filaments in electron microscopy of Alzheimer's Disease. Nature, 197(4863), 192-193. https://doi.org/10.1038/197192b0

Kim, D. H., Sarbassov, D. D., Ali, S. M., King, J. E., Latek, R. R., Erdjument-Bromage, H., ... Sabatini, D. M. (2002). mTOR interacts with raptor to form a nutrient-sensitive complex that signals to the cell growth machinery. Cell, 110(2), 163-175.

https://doi.org/10.1016/S0092-8674(02)00808-5

Kim, D.-H., Sarbassov, D. D., Ali, S. M., Latek, R. R., Guntur, K. V. P., Erdjument-Bromage, H., ... Sabatini, D. M. (2003). GbetaL, a positive regulator of the rapamycin-sensitive pathway required for the nutrient-sensitive interaction between raptor and mTOR. Molecular Cell, 11(4), 895-904. Retrieved from http://www.ncbi.nlm.nih.gov/pubmed/12718876

Kim, J. -w., Gao, P., Liu, Y.-C., Semenza, G. L., \& Dang, C. V. (2007). Hypoxia-Inducible Factor 1 and Dysregulated c-Myc Cooperatively Induce Vascular Endothelial Growth Factor and Metabolic Switches Hexokinase 2 and Pyruvate Dehydrogenase Kinase 1. Molecular and Cellular Biology, 27(21), 7381-7393. https://doi.org/10.1128/mcb.00440-07 
Kim, P. J., Plescia, J., Clevers, H., Fearon, E. R., \& Altieri, D. C. (2003). Survivin and molecular pathogenesis of colorectal cancer. Lancet, 362(9379), 205-209.

https://doi.org/10.1016/S0140-6736(03)13910-4

Kim, Y. H., Han, M.-E., \& Oh, S.-O. (2017). The molecular mechanism for nuclear transport and its application. Anatomy \& Cell Biology, 50(2), 77.

https://doi.org/10.5115/acb.2017.50.2.77

Kinoshita, E., Kinoshita-Kikuta, E., Takiyama, K., \& Koike, T. (2005). Phosphate-binding Tag, a New Tool to Visualize Phosphorylated Proteins. Molecular \& Cellular Proteomics, 5(4), 749-757. https://doi.org/10.1074/mcp.t500024-mcp200

Kiyatkin, A., Aksamitiene, E., Markevich, N. I., Borisov, N. M., Hoek, J. B., \& Kholodenko, B. N. (2006). Scaffolding protein Grb2-associated binder 1 sustains epidermal growth factorinduced mitogenic and survival signaling by multiple positive feedback loops. Journal of Biological Chemistry, 281(29), 19925-19938. https://doi.org/10.1074/jbc.M600482200

Kjaerulff, O., Brodin, L., \& Jung, A. (2011). The Structure and Function of Endophilin Proteins. Cell Biochemistry and Biophysics, 60(3), 137-154. https://doi.org/10.1007/s12013-0109137-5

Klauber-DeMore, N., Schulte, B. A., \& Wang, G. Y. (2018). Targeting MYC for triple-negative breast cancer treatment. Oncoscience, 5(5-6), 120-121.

https://doi.org/10.18632/oncoscience.414

Knies-Bamforth, U. E., Fox, S. B., Poulsom, R., Evan, G. I., \& Harris, A. L. (2004). c-Myc interacts with hypoxia to induce angiogenesis in vivo by a vascular endothelial growth factor-dependent mechanism. Cancer Research, 64(18), 6563-6570. https://doi.org/10.1158/0008-5472.CAN-03-3176

Knight, J. D. R., Choi, H., Gupta, G. D., Pelletier, L., Raught, B., Nesvizhskii, A. I., \& Gingras, A.-C. (2017). ProHits-viz: a suite of web tools for visualizing interaction proteomics data. Nature Methods, 14(7), 645-646. https://doi.org/10.1038/nmeth.4330

Knorr, R. L., Mizushima, N., \& Dimova, R. (2017). Fusion and scission of membranes: Ubiquitous topological transformations in cells. Traffic, 18(11), 758-761. https://doi.org/10.1111/tra.12509

Kobilka, B. K. (2007). G protein coupled receptor structure and activation. Biochimica et Biophysica Acta - Biomembranes, 1768(4), 794-807. https://doi.org/10.1016/j.bbamem.2006.10.021

Konagaya, Y., Terai, K., Hirao, Y., Takakura, K., Imajo, M., Kamioka, Y., ... Matsuda, M. (2017). A Highly Sensitive FRET Biosensor for AMPK Exhibits Heterogeneous AMPK Responses among Cells and Organs. Cell Reports, 21(9), 2628-2638. https://doi.org/10.1016/j.celrep.2017.10.113 
Kudo-Saito, C., Shirako, H., Takeuchi, T., \& Kawakami, Y. (2009). Cancer Metastasis Is Accelerated through Immunosuppression during Snail-Induced EMT of Cancer Cells. Cancer Cell, 15(3), 195-206. https://doi.org/10.1016/j.ccr.2009.01.023

Lakoduk, A. M., Roudot, P., Mettlen, M., Grossman, H. M., Schmid, S. L., \& Chen, P.-H. (2019). Mutant p53 amplifies a dynamin-1/APPL1 endosome feedback loop that regulates recycling and migration. The Journal of Cell Biology, jcb.201810183.

https://doi.org/10.1083/jcb.201810183

Lambert, S. A., Jolma, A., Campitelli, L. F., Das, P. K., Yin, Y., Albu, M., ... Weirauch, M. T. (2018). The Human Transcription Factors. Cell, 172(4), 650-665. https://doi.org/10.1016/j.cell.2018.01.029

Lamming, D. W., Ye, L., Katajisto, P., Goncalves, M. D., Saitoh, M., Stevens, D. M., ... Baur, J. A. (2012). Rapamycin-Induced Insulin Resistance Is Mediated by mTORC2 Loss and Uncoupled from Longevity. Science, 335(6076), 1638-1643. https://doi.org/10.1126/science. 1215135

Langemeyer, L., Fröhlich, F., \& Ungermann, C. (2018). Rab GTPase Function in Endosome and Lysosome Biogenesis. Trends in Cell Biology, 28(11), 957-970. https://doi.org/10.1016/j.tcb.2018.06.007

Larsson, C. (2006). Protein kinase $\mathrm{C}$ and the regulation of the actin cytoskeleton. Cellular Signalling, 18(3), 276-284. https://doi.org/10.1016/j.cellsig.2005.07.010

Lavin, D. P., White, M. F., \& Brazil, D. P. (2016). IRS proteins and diabetic complications. Diabetologia, 59(11), 2280-2291. https://doi.org/10.1007/s00125-016-4072-7

Laviola, L., Belsanti, G., Davalli, A. M., Napoli, R., Perrini, S., Weir, G. C., ... Giorgino, F. (2001). Effects of Streptozocin Diabetes and Diabetes Treatment by Islet Transplantation on In Vivo Insulin Signaling in Rat Heart. Diabetes, 50(12), 2709-2720. https://doi.org/10.2337/diabetes.50.12.2709

Lawrence, J. C., \& Roach, P. J. (1997). New insights into the role and mechanism of glycogen synthase activation by insulin. Diabetes, 46(4), 541-547. https://doi.org/10.2337/diab.46.4.541

Lee, K., Giltnane, J. M., Balko, J. M., Schwarz, L. J., Guerrero-Zotano, A. L., Hutchinson, K. E., ... Arteaga, C. L. (2017). MYC and MCL1 Cooperatively Promote Chemotherapy-Resistant Breast Cancer Stem Cells via Regulation of Mitochondrial Oxidative Phosphorylation. Cell Metabolism, 26(4), 633-647.e7. https://doi.org/10.1016/j.cmet.2017.09.009

Lee, T. I., \& Young, R. A. (2013). Transcriptional regulation and its misregulation in disease. Cell, 152(6), 1237-1251. https://doi.org/10.1016/j.cell.2013.02.014 
Lemmon, M. A., \& Schlessinger, J. (2010). Cell signaling by receptor tyrosine kinases. Cell, 141(7), 1117-1134. https://doi.org/10.1016/j.cell.2010.06.011

Lemmon, M. A., Schlessinger, J., \& Ferguson, K. M. (2014). The EGFR family: Not so prototypical receptor tyrosine kinases. Cold Spring Harbor Perspectives in Biology, 6(4). https://doi.org/10.1101/cshperspect.a020768

Leng, S., Zhang, W., Zheng, Y., Liberman, Z., Rhodes, C. J., Eldar-Finkelman, H., \& Sun, X. J. (2010). Glycogen synthase kinase $3 \beta$ mediates high glucose-induced ubiquitination and proteasome degradation of insulin receptor substrate 1. Journal of Endocrinology, 206(2), 171-181. https://doi.org/10.1677/JOE-09-0456

Lesort, M., Jope, R. S., \& Johnson, G. V. W. (1999). Insulin transiently increases tau phosphorylation: Involvement of glycogen synthase kinase- $3 \beta$ and Fyn tyrosine kinase. Journal of Neurochemistry, 72(2), 576-584. https://doi.org/10.1046/j.14714159.1999.0720576.x

Levin, R., Grinstein, S., \& Canton, J. (2016). The life cycle of phagosomes: formation, maturation, and resolution. Immunological Reviews, 273(1), 156-179. https://doi.org/10.1111/imr.12439

Lewis, T. S., Shapiro, P. S., \& Ahn, N. G. (2008). Signal Transduction through MAP Kinase Cascades, 49-139. https://doi.org/10.1016/s0065-230x(08)60765-4

Li, C. H., \& Tam, P. K. S. (1998). An iterative algorithm for minimum cross entropy thresholding. Pattern Recognition Letters, 19(8), 771-776. https://doi.org/10.1016/S01678655(98)00057-9

Li, Y., Xu, M., Ding, X., Yan, C., Song, Z., Chen, L., ... Yang, C. (2016). Protein kinase C controls lysosome biogenesis independently of mTORC1. Nature Cell Biology, 18(10), 1065-1077. https://doi.org/10.1038/ncb3407

Liberti, M. V., \& Locasale, J. W. (2016). The Warburg Effect: How Does it Benefit Cancer Cells? Trends in Biochemical Sciences, 41(3), 211-218. https://doi.org/10.1016/j.tibs.2015.12.001

Lim, J. P., \& Gleeson, P. A. (2011). Macropinocytosis: An endocytic pathway for internalising large gulps. Immunology and Cell Biology, 89(8), 836-843.

https://doi.org/10.1038/icb.2011.20

Lim, W. A., \& Pawson, T. (2010). Phosphotyrosine Signaling: Evolving a New Cellular Communication System. Cell, 142(5), 661-667. https://doi.org/10.1016/j.cell.2010.08.023

Liu, A. P., Aguet, F., Danuser, G., \& Schmid, S. L. (2010). Local clustering of transferrin receptors promotes clathrin-coated pit initiation. Journal of Cell Biology, 191(7), 13811393. https://doi.org/10.1083/jcb.201008117 
Liu, D. P., Song, H., \& Xu, Y. (2010). A common gain of function of p53 cancer mutants in inducing genetic instability. Oncogene, 29(7), 949-956.

https://doi.org/10.1038/onc.2009.376

Liu, F., Yang, X., Geng, M., \& Huang, M. (2018). Targeting ERK, an Achilles' Heel of the MAPK pathway, in cancer therapy. Acta Pharmaceutica Sinica B, 8(4), 552-562. https://doi.org/10.1016/j.apsb.2018.01.008

Liu, J. P., Sim, A. T. R., \& Robinson, P. J. (1994). Calcineurin inhibition of dynamin I GTPase activity coupled to nerve terminal depolarization. Science, 265(5174), 970-973. https://doi.org/10.1126/science.8052858

Liu, P., Gan, W., Chin, Y. R., Ogura, K., Guo, J., Zhang, J., .. Wei, W. (2015). PtdIns(3,4,5)P3Dependent Activation of the mTORC2 Kinase Complex. Cancer Discovery, 5(11), 1194 1209. https://doi.org/10.1158/2159-8290.CD-15-0460

Liu, S.-L., Wang, Z.-G., Hu, Y., Xin, Y., Singaram, I., Gorai, S., ... Cho, W. (2018).

Quantitative Lipid Imaging Reveals a New Signaling Function of Phosphatidylinositol-3,4Bisphophate: Isoform- and Site-Specific Activation of Akt. Molecular Cell, 71(6), 10921104.e5. https://doi.org/10.1016/j.molcel.2018.07.035

Liu, T., Yuan, X., \& Xu, D. (2016). Cancer-specific telomerase reverse transcriptase (Tert) promoter mutations: Biological and clinical implications. Genes, 7(7). https://doi.org/10.3390/genes7070038

Locasale, J. W., Grassian, A. R., Melman, T., Lyssiotis, C. A., Mattaini, K. R., Bass, A. J., ... Vander Heiden, M. G. (2011). Phosphoglycerate dehydrogenase diverts glycolytic flux and contributes to oncogenesis. Nature Genetics, 43(9), 869-874. https://doi.org/10.1038/ng.890

Locasale, J. W., \& Cantley, L. C. (2011). Metabolic flux and the regulation of mammalian cell growth. Cell Metabolism, 14(4), 443-451. https://doi.org/10.1016/j.cmet.2011.07.014

Lock, L. S., Royal, I., Naujokas, M. A., \& Park, M. (2000). Identification of an atypical Grb2 carboxyl-terminal SH3 domain binding site in Gab docking proteins reveals Grb2dependent and -independent recruitment of Gab1 to receptor tyorosine kinases. Journal of Biological Chemistry, 275(40), 31536-31545. https://doi.org/10.1074/jbc.M003597200

Long, X., Lin, Y., Ortiz-Vega, S., Yonezawa, K., \& Avruch, J. (2005). Rheb binds and regulates the mTOR kinase. Current Biology, 15(8), 702-713.

https://doi.org/10.1016/j.cub.2005.02.053

Lowenstein, E. J., Daly, R. J., Batzer, A. G., Li, W., Margolis, B., Lammers, R., ... Schlessinger, J. (1992). The SH2 and SH3 domain-containing protein GRB2 links receptor tyrosine kinases to ras signaling. Cell, 70(3), 431-442. https://doi.org/10.1016/0092-8674(92)90167$\mathrm{B}$ 
Lunt, S. Y., \& Vander Heiden, M. G. (2011). Aerobic Glycolysis: Meeting the Metabolic Requirements of Cell Proliferation. Annual Review of Cell and Developmental Biology, 27(1), 441-464. https://doi.org/10.1146/annurev-cellbio-092910-154237

Luo, M., \& Fu, L. W. (2014). Redundant kinase activation and resistance of EGFR-tyrosine kinase inhibitors. American Journal of Cancer Research, 4(6), 608-628.

Lüscher, B., \& Eisenman, R. N. (2015). C-Myc and C-Myb Protein Degradation: Effect of Metabolic Inhibitors and Heat Shock. Molecular and Cellular Biology, 8(6), 2504-2512. https://doi.org/10.1128/mcb.8.6.2504

Luzio, J. P., Pryor, P. R., \& Bright, N. A. (2007). Lysosomes: fusion and function. Nature Reviews. Molecular Cell Biology, 8(8), 622-632. https://doi.org/10.1038/nrm2217

Ly, P. T. T., Wu, Y., Zou, H., Wang, R., Zhou, W., Kinoshita, A., ... Song, W. (2013). Inhibition of GSK3 $\beta$-mediated BACE1 expression reduces Alzheimer-associated phenotypes. Journal of Clinical Investigation, 123(1), 224-235. https://doi.org/10.1172/JCI64516

M.A., B. (2005). Telomeres and human disease: Ageing, cancer and beyond. Nature Reviews Genetics, 6(8), 611-622. https://doi.org/10.1038/nrg1656 LK http://linksource.ebsco.com/linking.aspx?sid=EMBASE\&issn=14710056\&id=doi:10.1038 $\% 2$ Fnrg $1656 \&$ atitle $=$ Telomeres + and + human + disease $\% 3 \mathrm{~A}+$ Ageing $\% 2 \mathrm{C}+$ cancer + and + beyo nd\&stitle $=$ Nat. + Rev. + Gen.\&title $=$ Nature + Reviews + Genetics \&volume $=6 \&$ issue $=8 \&$ spage $=$ 611\&epage $=622$ \&aulast $=$ Blasco\&aufirst $=$ Maria + A.\&auinit $=$ M.A.\&aufull $=$ Blasco + M.A.\&c oden $=$ NRGAA\&isbn=\&pages $=611-622 \&$ date $=2005 \&$ auinit $1=$ M\&auinitm $=$ A

Ma, J., \& Cao, X. (2006). Regulation of Stat 3 nuclear import by importin $\alpha 5$ and importin $\alpha 7$ via two different functional sequence elements. Cellular Signalling, 18(8), 1117-1126. https://doi.org/10.1016/j.cellsig.2005.06.016

MacAulay, K., \& Woodgett, J. R. (2008). Targeting glycogen synthase kinase-3 (GSK-3) in the treatment of Type 2 diabetes. Expert Opinion on Therapeutic Targets, 12(10), 1265-1274. https://doi.org/10.1517/14728222.12.10.1265

Maehama, T., \& Dixon, J. E. (1998). The tumor suppressor, PTEN/MMAC1, dephosphorylates the lipid second messenger, phosphatidylinositol 3,4,5-trisphosphate. Journal of Biological Chemistry, 273(22), 13375-13378. https://doi.org/10.1074/jbc.273.22.13375

Maik-Rachline, G., Hacohen-Lev-Ran, A., \& Seger, R. (2019). Nuclear ERK: Mechanism of Translocation, Substrates, and Role in Cancer. International Journal of Molecular Sciences, 20(5), 1194. https://doi.org/10.3390/ijms20051194

Maire, C. L., \& Ligon, K. L. (2014). Molecular pathologic diagnosis of epidermal growth factor receptor. Neuro-Oncology, 16(suppl 8), viii1-viii6. https://doi.org/10.1093/neuonc/nou294 
Mancinelli, R., Carpino, G., Petrungaro, S., Mammola, C. L., Tomaipitinca, L., Filippini, A., ... Giampietri, C. (2017). Multifaceted roles of GSK-3 in cancer and autophagy-related diseases. Oxidative Medicine and Cellular Longevity, 2017. https://doi.org/10.1155/2017/4629495

Manning B.D., \& Cantley L.C. (2007). AKT/PKB Signaling: Navigating Downstream. Cell, 129, $1261-1274$.

Manning, B. D., \& Toker, A. (2017). AKT/PKB Signaling: Navigating the Network. Cell, 169(3), 381-405. https://doi.org/10.1016/j.cell.2017.04.001

Manoukian, A. S., \& Woodgett, J. R. (2002). Role of glycogen synthase kinase-3 in cancer: Regulation by Wnts and other signaling pathways. Advances in Cancer Research, 84, 203229. https://doi.org/10.1016/S0065-230X(02)84007-6

Marr, D., \& Hildreth, E. (1980). Theory of edge detection. Proceedings of the Royal Society of London Series B, Containing Papers of a Biological Character Royal Society (Great Britain), 207(1167), 187-217. https://doi.org/10.1098/rspb.1980.0020

Marshall, J. S., Warrington, R., Watson, W., \& Kim, H. L. (2018). An introduction to immunology and immunopathology. Allergy, Asthma \& Clinical Immunology, 14(S2), 49. https://doi.org/10.1186/s13223-018-0278-1

Martel, R. R., Klicius, J., \& Galet, S. (1977). Inhibition of the immune response by rapamycin, a new antifungal antibiotic. Canadian Journal of Physiology and Pharmacology, 55(1), 48-51. https://doi.org/10.1139/y77-007

Martina, J. A., Chen, Y., Gucek, M., \& Puertollano, R. (2012). MTORC1 functions as a transcriptional regulator of autophagy by preventing nuclear transport of TFEB. Autophagy, 8(6), 903-914. https://doi.org/10.4161/auto.19653

Matthew G. Vander Heiden, Lewis C. Cantley, \& Craig B. Thompson. (2009). Understanding the Warburg Effect: The Metabolic Requirements of Cell Proliferation. Science, 324(may), 1029-1033. https://doi.org/10.1126/science.1160809.Understanding

Mattoon, D. R., Lamothe, B., Lax, I., \& Schlessinger, J. (2004). The docking protein Gab1 is the primary mediator of EGF-stimulated activation of the PI-3K/Akt cell survival pathway. BMC Biology, 2. https://doi.org/10.1186/1741-7007-2-24

McCubrey, J. A., Davis, N. M., Abrams, S. L., Montalto, G., Cervello, M., Basecke, J., ... Steelman, L. S. (2014). Diverse roles of GSK-3: Tumor promoter-tumor suppressor, target in cancer therapy. Advances in Biological Regulation, 54, 176-196. https://doi.org/10.1016/j.jbior.2013.09.013

McLane, L. M., \& Corbett, A. H. (2009). Nuclear localization signals and human disease. IUBMB Life, 61(7), 697-706. https://doi.org/10.1002/iub.194 
McLaughlin, S., Wang, J., Gambhir, A., \& Murray, D. (2002). PIP 2 and Proteins: Interactions, Organization, and Information Flow. Annual Review of Biophysics and Biomolecular Structure, 31(1), 151-175. https://doi.org/10.1146/annurev.biophys.31.082901.134259

McMahon, H. T., \& Boucrot, E. (2011). Molecular mechanism and physiological functions of clathrin-mediated endocytosis. Nature Reviews Molecular Cell Biology, 12(8), 517-533. https://doi.org/10.1038/nrm3151

McNeela, E. A., Burke, Á., Neill, D. R., Baxter, C., Fernandes, V. E., Ferreira, D., ... Lavelle, E. C. (2010). Pneumolysin activates the NLRP3 inflammasome and promotes proinflammatory cytokines independently of TLR4. PLoS Pathogens, 6(11). https://doi.org/10.1371/journal.ppat.1001191

Meares, G. P., \& Jope, R. S. (2007). Resolution of the Nuclear Localization Mechanism of Glycogen Synthase Kinase-3. Journal of Biological Chemistry, 282(23), 16989-17001. https://doi.org/10.1074/jbc.M700610200

Medina, M., \& Avila, J. (2013). New insights into the role of glycogen synthase kinase-3 in Alzheimer's disease. Expert Opinion on Therapeutic Targets, 18(1), 69-77. https://doi.org/10.1517/14728222.2013.843670

Medina, M., \& Wandosell, F. (2011). Deconstructing GSK-3: The Fine Regulation of Its Activity. International Journal of Alzheimer's Disease, 2011, 1-12. https://doi.org/10.4061/2011/479249

Meşe, G., Richard, G., \& White, T. W. (2007). Gap Junctions: Basic Structure and Function. Journal of Investigative Dermatology, 127(11), 2516-2524. https://doi.org/10.1038/sj.jid.5700770

Mettlen, M., Stoeber, M., Loerke, D., Antonescu, C. N., Danuser, G., \& Schmid, S. L. (2009). Endocytic Accessory Proteins Are Functionally Distinguished by Their Differential Effects on the Maturation of Clathrin-coated Pits. Molecular Biology of the Cell, 20(14), 32513260. https://doi.org/10.1091/mbc.E09-03-0256

Mettlen, M., Chen, P., Srinivasan, S., Danuser, G., \& Schmid, S. L. (2018). Regulation of Clathrin-Mediated Endocytosis. Annual Review of Biochemistry, 87(1), annurev-biochem062917-012644. https://doi.org/10.1146/annurev-biochem-062917-012644

Mettlen, M., Stoeber, M., Loerke, D., Antonescu, C. N., Danuser, G., \& Schmid, S. L. (2009). Endocytic Accessory Proteins Are Functionally Distinguished by Their Differential Effects on the Maturation of Clathrin-coated Pits. Molecular Biology of the Cell, 20(14), 32513260. https://doi.org/10.1091/mbc.e09-03-0256

Miaczynska, M. (2013). Effects of membrane trafficking on signaling by receptor tyrosine kinases. Cold Spring Harbor Perspectives in Biology, 5(11). https://doi.org/10.1101/cshperspect.a009035 
Miaczynska, M., \& Stenmark, H. (2008). Mechanisms and functions of endocytosis. Journal of Cell Biology, 180(1), 7-11. https://doi.org/10.1083/jcb.200711073

Midha, A., Dearden, S., \& McCormack, R. (2015). EGFR mutation incidence in non-small-cell lung cancer of adenocarcinoma histology: a systematic review and global map by ethnicity (mutMapII). American Journal of Cancer Research, 5(9), 2892-2911. Retrieved from http://www.ncbi.nlm.nih.gov/pubmed/26609494\%0Ahttp://www.pubmedcentral.nih.gov/art iclerender.fcgi?artid=PMC4633915

Miller, D. M., Thomas, S. D., Islam, A., Muench, D., \& Sedoris, K. (2012). c-Myc and cancer metabolism. Clinical Cancer Research, 18(20), 5546-5553. https://doi.org/10.1158/10780432.CCR-12-0977

Mitin, N., Rossman, K. L., \& Der, C. J. (2005). Signaling interplay in ras superfamily function. Current Biology, 15(14). https://doi.org/10.1016/j.cub.2005.07.010

Mittal, V. (2018). Epithelial Mesenchymal Transition in Tumor Metastasis. Annual Review of Pathology: Mechanisms of Disease, 13(1), 395-412. https://doi.org/10.1146/annurevpathol-020117-043854

Mizushima, N., Kuma, A., Kobayashi, Y., Yamamoto, A., Matsubae, M., Takao, T., Natsume T., Ohsumi, Y., Yoshimori, T. (2003). Mouse Apg16L, a novel WD-repeat protein, targets to the autophagic isolation membrane with the Apg12-Apg5 conjugate. Journal of Cell Science, 116(9), 1679-1688. https://doi.org/10.1242/jcs.00381

Mizushima, N. (2007). Autophagy: Process and function. Genes and Development, 21(22), 2861-2873. https://doi.org/10.1101/gad.1599207

Morgillo, F., Della Corte, C. M., Fasano, M., \& Ciardiello, F. (2016). Mechanisms of resistance to EGFR-targeted drugs: Lung cancer. ESMO Open, 1(3). https://doi.org/10.1136/esmoopen-2016-000060

Morrison, D. K. (2012). MAP kinase pathways. Cold Spring Harbor Perspectives in Biology, 4(11), 479-500. https://doi.org/10.1101/cshperspect.a011254

Muller, P. A. J., \& Vousden, K. H. (2013). p53 mutations in cancer. Nature Cell Biology, 15(1), 2-8. https://doi.org/10.1038/ncb2641

Murray, D. H., Jahnel, M., Lauer, J., Avellaneda, M. J., Brouilly, N., Cezanne, A., ... Zerial, M. (2016). An endosomal tether undergoes an entropic collapse to bring vesicles together. Nature, 537(7618), 107-111. https://doi.org/10.1038/nature19326

Mutch, L. J., Howden, J. D., Jenner, E. P. L., Poulter, N. S., \& Rappoport, J. Z. (2014). Polarised Clathrin-Mediated Endocytosis of EGFR During Chemotactic Invasion. Traffic, 15(6), 648664. https://doi.org/10.1111/tra.12165 
Muz, B., de la Puente, P., Azab, F., \& Azab, A. K. (2015). The role of hypoxia in cancer progression, angiogenesis, metastasis, and resistance to therapy. Hypoxia, 83. https://doi.org/10.2147/hp.s93413

N., K., A., W., C., U., M., R., G., K., \& M., H. (2013). In vitro cell migration and invasion assays. Mutation Research - Reviews in Mutation Research, 752(1), 10-24. Retrieved from http://ovidsp.ovid.com/ovidweb.cgi?T $=\mathrm{JS} \& \mathrm{PAGE}=$ reference $\& \mathrm{D}=\mathrm{emed} 11 \& \mathrm{NEWS}=\mathrm{N} \& A N$ $=2013053213$

Nakamura, Y., \& Fukami, K. (2017). Regulation and physiological functions of mammalian phospholipase C. Journal of Biochemistry, 161(4), 315-321. https://doi.org/10.1093/jb/mvw094

Napolitano, G., Esposito, A., Choi, H., Matarese, M., Benedetti, V., Di Malta, C., ... Ballabio, A. (2018). mTOR-dependent phosphorylation controls TFEB nuclear export. Nature Communications, 9(1), 3312. https://doi.org/10.1038/s41467-018-05862-6

Naslavsky, N., \& Caplan, S. (2018). The enigmatic endosome - sorting the ins and outs of endocytic trafficking. Journal of Cell Science, 131(13), jcs216499. https://doi.org/10.1242/jcs.216499

Nawneet, K. K., Swati, P. J., Alok, V. J., Avinash, D. G., Prasad, D. C., Rahul, Y. D., \& Sharmila, A. B. (2009). Snail and Slug Mediate Radioresistance and Chemoresistance by Antagonizing p53-Mediated Apoptosis and Acquiring a Stem-Like Phenotype in Ovarian Cancer Cells. Stem Cells, 27(9), 2059-2068. Retrieved from http://dx.doi.org/10.1002/stem.154

Nelson, E. E., \& Guyer, A. E. (2014). Changes in cell and tissue organization in cancer of the breast and colon. Current Opinion in Cell Biology, 1(26), 87-95. https://doi.org/doi:10.1146/annurev-pathol-020117-043854

Neuzillet, C., Tijeras-Raballand, A., De Mestier, L., Cros, J., Faivre, S., \& Raymond, E. (2014). MEK in cancer and cancer therapy. Pharmacology and Therapeutics, 141(2), 160-171. https://doi.org/10.1016/j.pharmthera.2013.10.001

Nikolakaki, E., Coffer, P. J., Hemelsoet, R., Woodgett, J. R., \& Defize, L. H. (1993). Glycogen synthase kinase 3 phosphorylates Jun family members in vitro and negatively regulates their transactivating potential in intact cells. Oncogene, 8, 833-840.

Nikoulina, S. E., Ciaraldi, T. P., Mudaliar, S., Mohideen, P., Carter, L., \& Henry, R. R. (2000). Potential role of glycogen synthase kinase-3 in skeletal muscle insulin resistance of type 2 diabetes. Diabetes, 49(2), 263-271. https://doi.org/10.2337/diabetes.49.2.263

Nishizuka, Y. (1988). The molecular heterogeneiry of protein kinase C and its implications for cellualr recognition. Nature, 334, 661-665. 
Nojima, H., Tokunaga, C., Eguchi, S., Oshiro, N., Hidayat, S., Yoshino, K. I., ... Yonezawa, K. (2003). The mammalian target of rapamycin (mTOR) partner, raptor, binds the mTOR substrates p70 S6 kinase and 4E-BP1 through their TOR signaling (TOS) motif. Journal of Biological Chemistry, 278(18), 15461-15464. https://doi.org/10.1074/jbc.C200665200

O. Warburg. (1956). On the origin of cancer cells. Science, 123(3191), 309-314.

O’Conor, C. J., Chen, T., González, I., Cao, D., \& Peng, Y. (2018). Cancer stem cells in triplenegative breast cancer: a potential target and prognostic marker. Biomarkers in Medicine, 12(7), 813-820. https://doi.org/10.2217/bmm-2017-0398

Olmeda, D., Moreno-Bueno, G., Flores, J. M., Fabra, A., Portillo, F., \& Cano, A. (2007). SNAI1 is required for tumor growth and lymph node metastasis of human breast carcinoma MDAMB-231 cells. Cancer Research, 67(24), 11721-11731. https://doi.org/10.1158/00085472.CAN-07-2318

Osna, N. A., Donohue, T. M., \& Kharbanda, K. K. (2017). Alcoholic Liver Disease: Pathogenesis and Current Management. Alcohol Research : Current Reviews, 38(2), 147161. https://doi.org/10.1002/1098-2337(200011)26:6<413::AID-AB1>3.3.CO;2-H

Otto, T., \& Sicinski, P. (2017). Cell cycle proteins as promising targets in cancer therapy. Nature Reviews Cancer, 17(2), 93-115. https://doi.org/10.1038/nrc.2016.138

Ougolkov, A. V., Fernandez-Zapico, M. E., Savoy, D. N., Urrutia, R. A., \& Billadeau, D. D. (2005). Glycogen Synthase Kinase-3 $\beta$ Participates in Nuclear Factor $\kappa B-M e d i a t e d$ Gene Transcription and Cell Survival in Pancreatic Cancer Cells. Cancer Research, 65(6), 20762081. https://doi.org/10.1158/0008-5472.CAN-04-3642

Pálfy, M., Reményi, A., \& Korcsmáros, T. (2012). Endosomal crosstalk: Meeting points for signaling pathways. Trends in Cell Biology, 22(9), 447-456.

https://doi.org/10.1016/j.tcb.2012.06.004

Pamarthy, S., Kulshrestha, A., Katara, G. K., \& Beaman, K. D. (2018). The curious case of vacuolar ATPase: Regulation of signaling pathways. Molecular Cancer, 17(1). https://doi.org/10.1186/s12943-018-0811-3

Pan, Q., Bao, L. W., Teknos, T. N., \& Merajver, S. D. (2006). Targeted disruption of protein kinase $\mathrm{C} \varepsilon$ reduces cell invasion and motility through inactivation of RhoA and RhoC GTPases in head and neck squamous cell carcinoma. Cancer Research, 66(19), 9379-9384. https://doi.org/10.1158/0008-5472.CAN-06-2646

Panic, B., Whyte, J. R., Munro, S., Strochlic, T. I., Tong, a H., Boone, C., ... Zoncu, R. (2011). mTORC1 senses lysosomal amino acids through an inside-out mechanism that requires the valuolar H+-ATPase. Science, 334(November), 678-683.

https://doi.org/10.1126/science.1207056 
Pardo, O. E., Wellbrock, C., Khanzada, U. K., Aubert, M., Arozarena, I., Davidson, S., ... Seck1, M. J. (2006). FGF-2 protects small cell lung cancer cells from apoptosis through a complex involving PKCe, B-Raf and S6K2. EMBO Journal, 25(13), 3078-3088. https://doi.org/10.1038/sj.emboj.7601198

PARKER, P. J., CAUDWELL, F. B., \& COHEN, P. (1983). Glycogen Synthase from Rabbit Skeletal Muscle; Effect of Insulin on the State of phosphorylation of the Seven Phosphoserine Residues in vivo. European Journal of Biochemistry, 130(1), 227-234. https://doi.org/10.1111/j.1432-1033.1983.tb07140.x

Parmigiani, A., Nourbakhsh, A., Ding, B., Wang, W., Kim, Y. C., Akopiants, K., ... Budanov, A. V. (2014). Sestrins Inhibit mTORC1 Kinase Activation through the GATOR Complex. Cell Reports, 9(4), 1281-1291. https://doi.org/10.1016/j.celrep.2014.10.019

Pathi, N., Viswanath, S., Pathak, A., Rathore, A., \& Prukayastha, A. (2016). Receptor tyrosine kinase signaling pathways: a review. International Journal of Advances in Medicine, 783789. https://doi.org/10.18203/2349-3933.ijam20163714

Pavlova, N. N., \& Thompson, C. B. (2016). The Emerging Hallmarks of Cancer Metabolism. Cell Metabolism, 23(1), 27-47. https://doi.org/10.1016/j.cmet.2015.12.006

Pearce, L. R., Huang, X., Boudeau, J., Pawłowski, R., Wullschleger, S., Deak, M., ... Alessi, D. R. (2007). Identification of Protor as a novel Rictor-binding component of mTOR complex2. The Biochemical Journal, 405(3), 513-522. https://doi.org/10.1042/BJ20070540

Peeters, M., Karthaus, M., Rivera, F., Terwey, J.-H., \& Douillard, J.-Y. (2015). Panitumumab in Metastatic Colorectal Cancer: The Importance of Tumour RAS Status. Drugs, 75(7), 731748. https://doi.org/10.1007/s40265-015-0386-X

Peinado, H. (2004). Snail and E47 repressors of E-cadherin induce distinct invasive and angiogenic properties in vivo. Journal of Cell Science, 117(13), 2827-2839. https://doi.org/10.1242/jcs.01145

Perona, R. (2006). Cell signalling: Growth factors and tyrosine kinase receptors. Clinical and Translational Oncology, 8(2), 77-82. https://doi.org/10.1007/s12094-006-0162-1

Peterson, T. R., Laplante, M., Thoreen, C. C., Sancak, Y., Kang, S. A., Kuehl, W. M., ... Sabatini, D. M. (2009). DEPTOR Is an mTOR Inhibitor Frequently Overexpressed in Multiple Myeloma Cells and Required for Their Survival. Cell, 137(5), 873-886. https://doi.org/10.1016/j.cell.2009.03.046

Petit, C. S., Roczniak-Ferguson, A., \& Ferguson, S. M. (2013). Recruitment of folliculin to lysosomes supports the amino acid-dependent activation of rag gtpases. Journal of Cell Biology, 202(7), 1107-1122. https://doi.org/10.1083/jcb.201307084 
Platt, F. M., D’Azzo, A., Davidson, B. L., Neufeld, E. F., \& Tifft, C. J. (2018). Lysosomal storage diseases. Nature Reviews Disease Primers, 4(1), 27. https://doi.org/10.1038/s41572018-0025-4

Polakis, P. (2002). Casein Kinase 1: A Wnt'er of Disconnect. Current Biology, 12(14), R499R501. https://doi.org/10.1016/S0960-9822(02)00969-7

Polo, S., Sigismund, S., Argenzio, E., Tosoni, D., Cavallaro, E., \& Di Fiore, P. P. (2008). Clathrin-Mediated Internalization Is Essential for Sustained EGFR Signaling but Dispensable for Degradation. Developmental Cell, 15(2), 209-219. https://doi.org/10.1016/j.devcel.2008.06.012

Porstmann, T., Santos, C. R., Griffiths, B., Cully, M., Wu, M., Leevers, S., ... Schulze, A. (2008). SREBP Activity Is Regulated by mTORC1 and Contributes to Akt-Dependent Cell Growth. Cell Metabolism, 8(3), 224-236. https://doi.org/10.1016/j.cmet.2008.07.007

Possemato, R., Marks, K. M., Shaul, Y. D., Pacold, M. E., Kim, D., Birsoy, K., ... Sabatini, D. M. (2011). Functional genomics reveal that the serine synthesis pathway is essential in breast cancer. Nature, 476(7360), 346-350. https://doi.org/10.1038/nature10350

Poteryaev, D., Datta, S., Ackema, K., Zerial, M., \& Spang, A. (2010). Identification of the switch in early-to-late endosome transition. Cell, 141(3), 497-508.

https://doi.org/10.1016/j.cell.2010.03.011

Qi, D., \& Young, L. H. (2015). AMPK: Energy sensor and survival mechanism in the ischemic heart. Trends in Endocrinology and Metabolism, 26(8), 422-429. https://doi.org/10.1016/j.tem.2015.05.010

Qing, G., Skuli, N., Mayes, P. A., Pawel, B., Martinez, D., Maris, J. M., \& Simon, M. C. (2010). Combinatorial regulation of neuroblastoma tumor progression by N-Myc and hypoxia inducible factor HIF-1 $\alpha$. Cancer Research, 70(24), 10351-10361. https://doi.org/10.1158/0008-5472.CAN-10-0740

Ra, S., \& Dm, S. (2017). mTOR Signaling in Growth , Metabolism , and Disease . Cell, 168(6), 960-976. https://doi.org/10.1016/j.cell.2017.02.004.mTOR

Rebsamen, P., Stasyk, de A., Galluccio, K., Snijder, F., Rudashevskaya, B., Scorzoni, F., ... Huber, S.-F. (2015). SLC38A9 is a component of the lysosomal amino acid sensing machinery that controls mTORC1. Nature, 519(7544), 477-481.

Reis, C. R., Chen, P.-H., Srinivasan, S., Aguet, F., Mettlen, M., \& Schmid, S. L. (2015). Crosstalk between Akt/GSK3 signaling and dynamin-1 regulates clathrin-mediated endocytosis. The EMBO Journal, 34(16), 2132-2146.

https://doi.org/10.15252/embj.201591518 
Reis, C. R., Chen, P.-H., Bendris, N., \& Schmid, S. L. (2017). TRAIL-death receptor endocytosis and apoptosis are selectively regulated by dynamin-1 activation. Proceedings of the National Academy of Sciences, 114(3), 504-509. https://doi.org/10.1073/pnas.1615072114

Robert, F., C., L. M., Lars-Gustav, L., \& B., S. H. (2003). The G-Protein-Coupled Receptors in the Human Genome Form Five Main Families . Phylogenetic Analysis , Paralogon Groups , and Fingerprints. Molecular Pharmacology, 63(6), 1256-1272.

https://doi.org/10.1124/mol.63.6.1256

Roczniak-Ferguson, A., Petit, C. S., Froehlich, F., Qian, S., Ky, J., Angarola, B., ... Ferguson, S. M. (2012). The Transcription Factor TFEB Links mTORC1 Signaling to Transcriptional Control of Lysosome Homeostasis. Science Signaling, 5(228), ra42-ra42. https://doi.org/10.1126/scisignal.2002790

Roepstorff, K., Grandal, M. V., Henriksen, L., Knudsen, S. L. J., Lerdrup, M., Grøvdal, L., ... Van Deurs, B. (2009). Differential effects of EGFR ligands on endocytic sorting of the receptor. Traffic, 10(8), 1115-1127. https://doi.org/10.1111/j.1600-0854.2009.00943.x

Romanov, J., Walczak, M., Ibiricu, I., Schüchner, S., Ogris, E., Kraft, C., \& Martens, S. (2012). Mechanism and functions of membrane binding by the Atg5-Atg12/Atg16 complex during autophagosome formation. EMBO Journal, 31(22), 4304-4317. https://doi.org/10.1038/emboj.2012.278

Rosales, C., \& Uribe-Querol, E. (2017). Phagocytosis: A Fundamental Process in Immunity. BioMed Research International, 2017. https://doi.org/10.1155/2017/9042851

Roskoski Jr, R. (2014). The ErbB/HER family of protein-tyrosine kinases and cancer. Pharmacological Research, 79, 34-74. https://doi.org/http://dx.doi.org/10.1016/j.phrs.2013.11.002

Rosner, M., Freilinger, A., \& Hengstschläger, M. (2007). Akt regulates nuclear/cytoplasmic localization of tuberin. Oncogene, 26(4), 521-531. https://doi.org/10.1038/sj.onc.1209812

Rosner, M., \& Hengstschläger, M. (2008). Cytoplasmic and nuclear distribution of the protein complexes mTORC1 and mTORC2: Rapamycin triggers dephosphorylation and delocalization of the mTORC 2 components rictor and sin1. Human Molecular Genetics, 17(19), 2934-2948. https://doi.org/10.1093/hmg/ddn192

Ross, E., Ata, R., Thavarajah, T., Medvedev, S., Bowden, P., Marshall, J. G., \& Antonescu, C. N. (2015). AMP-Activated Protein Kinase Regulates the Cell Surface Proteome and Integrin Membrane Traffic. PLOS ONE, 10(5), e0128013. https://doi.org/10.1371/journal.pone.0128013 
Rosselli-Murai, L. K., Yates, J. A., Yoshida, S., Bourg, J., Ho, K. K. Y., White, M., ... Liu, A. P. (2018). Loss of PTEN promotes formation of signaling-capable clathrin-coated pits. Journal of Cell Science, 131(8). https://doi.org/10.1242/jcs.208926

Roux, K. J., Kim, D. I., Burke, B., \& May, D. G. (2018). BioID: A Screen for Protein-Protein Interactions. Current Protocols in Protein Science, 91, 19.23.1-19.23.15. https://doi.org/10.1002/cpps.51

Roy, N., Bordoloi, D., Monisha, J., Padmavathi, G., Kotoky, J., Golla, R., \& Kunnumakkara, A. (2016). Specific Targeting of Akt Kinase Isoforms: Taking the Precise Path for Prevention and Treatment of Cancer. Current Drug Targets, 18(4), 421-435. https://doi.org/10.2174/1389450117666160307145236

RYLATT, D. B., AITKEN, A., BILHAM, T., CONDON, G. D., EMBI, N., \& COHEN, P. (1980). Glycogen Synthase from Rabbit Skeletal Muscle: Amino Acid Sequence at the Sites Phosphorylated by Glycogen Synthase Kinase-3, and Extension of the N-Terminal Sequence Containing the Site Phosphorylated by Phosphorylase Kinase. European Journal of Biochemistry, 107(2), 529-537. https://doi.org/10.1111/j.1432-1033.1980.tb06060.x

S., A., R.H., B., \& G., A. (2011). The Possible Involvement of Glycogen Synthase Kinase-3 (GSK-3) in Diabetes, Cancer and Central Nervous System Diseases. Current Pharmaceutical Design, 17(22), 2264-2277. https://doi.org/10.2174/138161211797052484

Sabatini, D. M., Erdjument-Bromage, H., Lui, M., Tempst, P., \& Snyder, S. H. (1994). RAFT1: A mammalian protein that binds to FKBP12 in a rapamycin-dependent fashion and is homologous to yeast TORs. Cell, 78(1), 35-43. https://doi.org/10.1016/00928674(94)90570-3

Sabers, C. J., Martin, M. M., Brunn, G. J., Williams, J. M., Dumont, F. J., Wiederrecht, G., \& Abraham, R. T. (1995). Isolation of a Protein Target of the FKBP12-Rapamycin Complex in Mammalian Cells. Journal of Biological Chemistry, 270(2), 815-822. https://doi.org/10.1074/jbc.270.2.815

Saheki, Y., \& De Camilli, P. (2012). Synaptic Vesicle Endocytosis. Cold Spring Harbor Perspectives in Biology, 4(9), a005645-a005645. https://doi.org/10.1101/cshperspect.a005645

Samanta, D., Gilkes, D. M., Chaturvedi, P., Xiang, L., \& Semenza, G. L. (2014). Hypoxiainducible factors are required for chemotherapy resistance of breast cancer stem cells. Proceedings of the National Academy of Sciences, 111(50), E5429-E5438. https://doi.org/10.1073/pnas.1421438111

Sancak, Y., Bar-Peled, L., Zoncu, R., Markhard, A. L., Nada, S., \& Sabatini, D. M. (2010). Ragulator-rag complex targets mTORC1 to the lysosomal surface and is necessary for its activation by amino acids. Cell, 141(2), 290-303. https://doi.org/10.1016/j.cell.2010.02.024 
Sancak, Y., Peterson, T. R., Shaul, Y. D., Lindquist, R. A., Thoreen, C. C., Bar-Peled, L., \& Sabatini, D. M. (2008). The rag GTPases bind raptor and mediate amino acid signaling to mTORC1. Science, 320(5882), 1496-1501. https://doi.org/10.1126/science.1157535

Sancak, Y., Thoreen, C. C., Peterson, T. R., Lindquist, R. A., Kang, S. A., Spooner, E., ... Sabatini, D. M. (2007). PRAS40 Is an Insulin-Regulated Inhibitor of the mTORC1 Protein Kinase. Molecular Cell, 25(6), 903-915. https://doi.org/10.1016/j.molcel.2007.03.003

Sandvig, K., Kavaliauskiene, S., \& Skotland, T. (2018). Clathrin-independent endocytosis: an increasing degree of complexity. Histochemistry and Cell Biology, 150(2), 107-118. https://doi.org/10.1007/s00418-018-1678-5

Sarbassov, D. D., Ali, S. M., Kim, D.-H., Guertin, D. A., Latek, R. R., Erdjument-Bromage, H., ... Sabatini, D. M. (2004). Rictor, a novel binding partner of mTOR, defines a rapamycininsensitive and raptor-independent pathway that regulates the cytoskeleton. Current Biology : CB, 14(14), 1296-1302. https://doi.org/10.1016/j.cub.2004.06.054

Sarbassov, D. D., Guertin, D. A., Ali, S. M., \& Sabatini, D. M. (2005). Phosphorylation and regulation of Akt/PKB by the rictor-mTOR complex. Science, 307(5712), 1098-1101. https://doi.org/10.1126/science. 1106148

Saric, A., Hipolito, V. E. B., Kay, J. G., Canton, J., Antonescu, C. N., \& Botelho, R. J. (2016). mTOR controls lysosome tubulation and antigen presentation in macrophages and dendritic cells. Molecular Biology of the Cell, 27(2), 321-333. https://doi.org/10.1091/mbc.e15-050272

Satoo, K., Noda, N. N., Kumeta, H., Fujioka, Y., Mizushima, N., Ohsumi, Y., \& Inagaki, F. (2009). The structure of Atg4B-LC3 complex reveals the mechanism of LC3 processing and delipidation during autophagy. EMBO Journal, 28(9), 1341-1350.

https://doi.org/10.1038/emboj.2009.80

Sawano, A., Takayama, S., Matsuda, M., \& Miyawaki, A. (2002). Lateral propagation of EGF signaling after local stimulation is dependent on receptor density. Developmental Cell, 3(2), 245-257. https://doi.org/10.1016/S1534-5807(02)00224-1

Saxton, R. A., Chantranupong, L., Knockenhauer, K. E., Schwartz, T. U., \& Sabatini, D. M. (2016). Mechanism of arginine sensing by CASTOR1 upstream of mTORC1. Nature, 536(7615), 229-233. https://doi.org/10.1038/nature19079

Schalm, S. S., Fingar, D. C., Sabatini, D. M., \& Blenis, J. (2003). TOS motif-mediated raptor binding regulates 4E-BP1 multisite phosphorylation and function. Current Biology, 13(10), 797-806. https://doi.org/10.1016/S0960-9822(03)00329-4

Schenck, A., Goto-Silva, L., Collinet, C., Rhinn, M., Giner, A., Habermann, B., ... Zerial, M. (2008). The Endosomal Protein Appl1 Mediates Akt Substrate Specificity and Cell Survival 
in Vertebrate Development. Cell, 133(3), 486-497.

https://doi.org/10.1016/j.cell.2008.02.044

Schmid, E. M., \& McMahon, H. T. (2007). Integrating molecular and network biology to decode endocytosis. Nature, 448(7156), 883-888. https://doi.org/10.1038/nature06031

Schmidt, M. H. H., Furnari, F. B., Cavenee, W. K., \& Bogler, O. (2003). Epidermal growth factor receptor signaling intensity determines intracellular protein interactions, ubiquitination, and internalization. Proceedings of the National Academy of Sciences, 100(11), 6505-6510. https://doi.org/10.1073/pnas.1031790100

Schultze, S. M., Jensen, J., Hemmings, B. A., Tschopp, O., \& Niessen, M. (2011). Promiscuous affairs of PKB/AKT isoforms in metabolism. Archives of Physiology and Biochemistry, 117(2), 70-77. https://doi.org/10.3109/13813455.2010.539236

Sears, R. (2000). Multiple Ras-dependent phosphorylation pathways regulate Myc protein stability. Genes \& Development, 14(19), 2501-2514. https://doi.org/10.1101/gad.836800

Sears, R. C. (2004). The life cycle of C-Myc: from synthesis to degradation. Cell Cycle (Georgetown, Tex.), 3(9), 1133-1137. Retrieved from http://www.ncbi.nlm.nih.gov/pubmed/15467447

Sears, R., Nuckolls, F., Haura, E., Taya, Y., Tamai, K., \& Nevins, J. R. (2000). Multiple Rasdependent phosphorylation pathways regulate Myc protein stability. Genes \& Development, 14(19), 2501-2514. https://doi.org/10.1101/gad.836800

Sekiya, F., Poulin, B., Kim, Y. J., \& Rhee, S. G. (2004). Mechanism of tyrosine phosphorylation and activation of phospholipase c- $\gamma 1$. Tyrosine 783 phosphorylation is not sufficient for lipase activation. Journal of Biological Chemistry, 279(31), 32181-32190.

https://doi.org/10.1074/jbc.M405116200

Sekiya, S., \& Suzuki, A. (2011). Glycogen synthase kinase 3 -dependent Snail degradation directs hepatocyte proliferation in normal liver regeneration. Proceedings of the National Academy of Sciences, 108(27), 11175-11180. https://doi.org/10.1073/pnas.1016122108

Sen, A., Madhivanan, K., Mukherjee, D., \& Claudio Aguilar, R. (2012). The epsin protein family: Coordinators of endocytosis and signaling. Biomolecular Concepts, 3(2), 117-126. https://doi.org/10.1515/bmc-2011-0060

Sen, R., \& Baltimore, D. (2013). The complexity of NF-אB signaling in inflammation and cancer. Molecular Cancer, 12(86). https://doi.org/10.1016/0092-8674(86)90346-6

Settembre, C., Zoncu, R., Medina, D. L., Vetrini, F., Erdin, S., Erdin, S., ... Ballabio, A. (2012). A lysosome-to-nucleus signalling mechanism senses and regulates the lysosome via mTOR and TFEB. EMBO Journal, 31(5), 1095-1108. https://doi.org/10.1038/emboj.2012.32 
Shen, K., Huang, R. K., Brignole, E. J., Condon, K. J., Valenstein, M. L., Chantranupong, L., ... Sabatini, D. M. (2018). Architecture of the human GATOR1 and GATOR1-Rag GTPases complexes. Nature, 556(7699), 64-69. https://doi.org/10.1038/nature26158

Shen, K., Valenstein, M. L., Gu, X., \& Sabatini, D. M. (2019). Arg78 of Nprl2 catalyzes GATOR1-stimulated GTP hydrolysis by the Rag GTPases. Journal of Biological Chemistry, jbc.AC119.007382. https://doi.org/10.1074/jbc.ac119.007382

Sherr, C. J., \& Roberts, J. M. (2004). Living with or without cyclins and cyclin-dependent kinases. Genes and Development, 18(22), 2699-2711. https://doi.org/10.1101/gad.1256504

Shi, C., Shakey, Q., Shor, B., Follettie, M., Toral-Barza, L., Wu, J., \& Yu, K. (2010).

Requirement of the mTOR Kinase for the Regulation of Mafl Phosphorylation and Control of RNA Polymerase III-dependent Transcription in Cancer Cells. Journal of Biological Chemistry, 285(20), 15380-15392. https://doi.org/10.1074/jbc.m109.071639

Shukuya, T., Takahashi, T., Kaira, R., Ono, A., Nakamura, Y., Tsuya, A., ... Yamamoto, N. (2011). Efficacy of gefitinib for non-adenocarcinoma non-small-cell lung cancer patients harboring epidermal growth factor receptor mutations: A pooled analysis of published reports. Cancer Science, 102(5), 1032-1037. https://doi.org/10.1111/j.13497006.2011.01887.x

Sigismund, S., Avanzato, D., \& Lanzetti, L. (2018). Emerging functions of the EGFR in cancer. Molecular Oncology, 12(1), 3-20. https://doi.org/10.1002/1878-0261.12155

Singh, A. B., \& Harris, R. C. (2005). Autocrine, paracrine and juxtacrine signaling by EGFR ligands. Cellular Signalling, 17(10), 1183-1193. https://doi.org/10.1016/j.cellsig.2005.03.026

Singh, D., Attri, B., Gill, R., \& Bariwal, J. (2016). Review On EGFR Inhibitors: Critical Updates. Mini-Reviews in Medicinal Chemistry, 16(999), 1-1. https://doi.org/10.2174/1389557516666160804162457

Slepnev, V. I., Ochoa, G. C., Butler, M. H., Grabs, D., \& De Camilli, P. (1998). Role of phosphorylation in regulation of the assembly of endocytic coat complexes. Science, 281(5378), 821-824. https://doi.org/10.1126/science.281.5378.821

Smillie, K. J., \& Cousin, M. A. (2011). The Role of GSK3 in Presynaptic Function. International Journal of Alzheimer's Disease, 2011, 1-8. https://doi.org/10.4061/2011/263673

Smith, K. P., Byron, M., O’Connell, B. C., Tam, R., Schorl, C., Guney, I., ... Lawrence, J. B. (2004). c-Myc localization within the nucleus: Evidence for association with the PML nuclear body. Journal of Cellular Biochemistry, 93(6), 1282-1296.

https://doi.org/10.1002/jcb.20273 
Smith, L., Bryan, S., De, P., Rahal, R., Shaw, A., Turner, D., ... Dixon, M. (2018). Canadian Cancer Statistics: A 2018 special report on cancer incidence by stage. Retrieved from http://www.cancer.ca/ /media/cancer.ca/CW/cancer information/cancer 101/Canadian cancer statistics/Canadian-Cancer-Statistics-2018-EN.pdf?la=en

Song, H., Hollstein, M., \& Xu, Y. (2007). p53 gain-of-function cancer mutants induce genetic instability by inactivating ATM. Nature Cell Biology, 9(5), 573-580. https://doi.org/10.1038/ncb1571

Sönnichsen, B., De Renzis, S., Nielsen, E., Rietdorf, J., \& Zerial, M. (2000). Distinct membrane domains on endosomes in the recycling pathway visualized by multicolor imaging of Rab4, Rab5, and Rab11. Journal of Cell Biology, 149(4), 901-913. https://doi.org/10.1083/jcb.149.4.901

Sorkin, A., \& Goh, L. K. (2009). Endocytosis and intracellular trafficking of ErbBs. Experimental Cell Research, 315(4), 683-696. https://doi.org/10.1016/j.yexcr.2008.07.029

Srinivasan, S., Burckhardt, C. J., Bhave, M., Chen, Z., Chen, P. H., Wang, X., ... Schmid, S. L. (2018). A noncanonical role for dynamin-1 in regulating early stages of clathrin-mediated endocytosis in non-neuronal cells. PLoS Biology, 16(4). https://doi.org/10.1371/journal.pbio.2005377

Stadler, S., Vincent, C. T., Fedorov, V. D., Patsialou, A., Cherrington, B. D., Wakshlag, J. J., ... Allis, C. D. (2013). Dysregulation of PAD4-mediated citrullination of nuclear GSK3 $\beta$ activates TGF- $\beta$ signaling and induces epithelialto-mesenchymal transition in breast cancer cells. Proceedings of the National Academy of Sciences, 110(40), 16283-16283. https://doi.org/10.1073/pnas.1316463110

Stambolic, V., \& Woodgett, J. R. (1994). Mitogen inactivation of glycogen synthase kinase-3 $\beta$ in intact cells via serine 9 phosphorylation. Biochemical Journal, 303(3), 701-704. https://doi.org/10.1042/bj3030701

Stambolic, V., \& Woodgett, J. R. (2006). Functional distinctions of protein kinase B/Akt isoforms defined by their influence on cell migration. Trends in Cell Biology, 16(9), 461466. https://doi.org/10.1016/j.tcb.2006.07.001

Statistics Canada. (2014). Canadian Cancer Statistics. Canadian Cancer Society, 2014, 1-132. https://doi.org/0835-2976

Stenmark, H., Simonsen, A., Lippe', R., Christoforidis, S., Gaullier, J.-M., Brech, A., ... Zerial, M. (1998). EEA1 links PI(3)K function to Rab5 regulation of endosome fusion. Nature, 394(6692), 494-498. https://doi.org/10.1038/28879

Strambio-De-Castillia, C., Niepel, M., \& Rout, M. P. (2010). The nuclear pore complex: Bridging nuclear transport and gene regulation. Nature Reviews Molecular Cell Biology, 11(7), 490-501. https://doi.org/10.1038/nrm2928 
Stretton, C., Hoffmann, T. M., Munson, M. J., Prescott, A., Taylor, P. M., Ganley, I. G., \& Hundal, H. S. (2015). GSK3-mediated raptor phosphorylation supports amino-aciddependent mTORC1-directed signalling. Biochemical Journal, 470(2), 207-221. https://doi.org/10.1042/bj20150404

Stüven, T., Hartmann, E., \& Görlich, D. (2003). Exportin 6: A novel nuclear export receptor that is specific for profilin-actin complexes. EMBO Journal, 22(21), 5928-5940. https://doi.org/10.1093/emboj/cdg565

Sugiyama, M. G., Fairn, G. D., \& Antonescu, C. N. (2019). Akt-ing Up Just About Everywhere: Compartment-Specific Akt Activation and Function in Receptor Tyrosine Kinase Signaling. Frontiers in Cell and Developmental Biology, 7. https://doi.org/10.3389/fcell.2019.00070

Sun, Q., Fan, W., \& Zhong, Q. (2009). Regulation of Beclin 1 in autophagy. Autophagy, 5(5), 713-716. Retrieved from

http://www.ncbi.nlm.nih.gov/pubmed/19372752\%0Ahttp://www.pubmedcentral.nih.gov/art iclerender.fcgi?artid=PMC2789700

Suzuki, T., Bridges, D., Nakada, D., Skiniotis, G., Morrison, S. J., Lin, J. D., ... Inoki, K. (2013). Inhibition of AMPK catabolic action by GSK3. Molecular Cell, 50(3), 407-419. https://doi.org/10.1016/j.molcel.2013.03.022

Swanson, J. A. (2008). Shaping cups into phagosomes and macropinosomes. Nature Reviews Molecular Cell Biology, 9(8), 639-649. https://doi.org/10.1038/nrm2447

Taelman, V. F., Plouhinec, J.-L., Vorwald, P. P., Sabatini, D. D., Gumper, I., Dobrowolski, R., ... Fuentealba, L. C. (2010). Wnt Signaling Requires Sequestration of Glycogen Synthase Kinase 3 inside Multivesicular Endosomes. Cell, 143(7), 1136-1148. https://doi.org/10.1016/j.cell.2010.11.034

Taelman, V. F., Dobrowolski, R., Plouhinec, J.-L., Fuentealba, L. C., Vorwald, P. P., Gumper, I., ... De Robertis, E. M. (2010). Wnt Signaling Requires Sequestration of Glycogen Synthase Kinase 3 inside Multivesicular Endosomes. Cell, 143(7), 1136-1148. https://doi.org/10.1016/j.cell.2010.11.034

Taki, M., Abiko, K., Baba, T., Hamanishi, J., Yamaguchi, K., Murakami, R., .. Matsumura, N. (2018). Snail promotes ovarian cancer progression by recruiting myeloid-derived suppressor cells via CXCR2 ligand upregulation. Nature Communications, 9(1). https://doi.org/10.1038/s41467-018-03966-7

Tan, M. L., Tan, H. K., \& Tengku Muhammad, T. S. (2015). Apoptosis and cancer. Cancer Immunology: A Translational Medicine Context, 209-242. https://doi.org/10.1007/978-3662-44006-3_12 
Tanida, I., Ueno, T., \& Kominami, E. (2004). LC3 conjugation system in mammalian autophagy. International Journal of Biochemistry and Cell Biology, 36(12), 2503-2518. https://doi.org/10.1016/j.biocel.2004.05.009

Taylor, M. J., Perrais, D., \& Merrifield, C. J. (2011). A high precision survey of the molecular dynamics of mammalian clathrin-mediated endocytosis. PLoS Biology, 9(3). https://doi.org/10.1371/journal.pbio.1000604

Tee, A. R., Manning, B. D., Roux, P. P., Cantley, L. C., \& Blenis, J. (2003). Tuberous Sclerosis Complex Gene Products, Tuberin and Hamartin, Control mTOR Signaling by Acting as a GTPase-Activating Protein Complex toward Rheb. Current Biology, 13(15), 1259-1268. https://doi.org/10.1016/S0960-9822(03)00506-2

Teo, G., Liu, G., Zhang, J., Nesvizhskii, A. I., Gingras, A.-C., \& Choi, H. (2014). SAINTexpress: improvements and additional features in Significance Analysis of INTeractome software. Journal of Proteomics, 100, 37-43. https://doi.org/10.1016/j.jprot.2013.10.023

Thaiss, C. A., Levy, M., Itav, S., \& Elinav, E. (2016). Integration of Innate Immune Signaling. Trends in Immunology, 37(2), 84-101. https://doi.org/10.1016/j.it.2015.12.003

Thedieck, K., Polak, P., Kim, M. L., Molle, K. D., Cohen, A., Jenö, P., ... Hall, M. N. (2007). PRAS40 and PRR5-Like Protein Are New mTOR Interactors that Regulate Apoptosis. PLoS ONE, 2(11), e1217. https://doi.org/10.1371/journal.pone.0001217

Thornton, T. M., Pedraza-Alva, G., Deng, B., Wood, C. D., Aronshtam, A., Clements, J. L., ... Rincon, M. (2008). Phosphorylation by p38 MAPK as an Alternative Pathway for GSK3 Inactivation. Science, 320(5876), 667-670. https://doi.org/10.1126/science.1156037

Thornton, T. M., Pedraza-Alva, G., Deng, B., Wood, C. D., Aronshtam, A., Clements, J. L., ... Rincon, M. (2008). Phosphorylation by p38 MAPK as an alternative pathway for GSK3 $\beta$ inactivation. Science, 320(5876), 667-670. https://doi.org/10.1126/science.1156037

Tokarev, A. A., Alfonso, A., \& Segev, N. (2009). Overview of Intracellular Compartments and Trafficking Pathways. Trafficking Inside Cells, 3-14. https://doi.org/10.1007/978-0-38793877-6_1

Traub, L. M., \& Bonifacino, J. S. (2013). Cargo recognition in clathrin-mediated endocytosis. Cold Spring Harbor Perspectives in Biology, 5(11). https://doi.org/10.1101/cshperspect.a016790

Tsai, F.-C., Kuo, G.-H., Chang, S.-W., \& Tsai, P.-J. (2015). Ca 2+ Signaling in Cytoskeletal Reorganization, Cell Migration, and Cancer Metastasis. BioMed Research International, 2015, 1-13. https://doi.org/10.1155/2015/409245 
Tsang, C. K., Liu, H., \& Zheng, X. F. S. (2010). mTOR binds to the promoters of RNA polymerase I- And III-transcribed genes. Cell Cycle, 9(5), 953-957.

https://doi.org/10.4161/cc.9.5.10876

Tsou, P., Zheng, B., Hsu, C. H., Sasaki, A. T., \& Cantley, L. C. (2011). A fluorescent reporter of AMPK activity and cellular energy stress. Cell Metabolism, 13(4), 476-486.

https://doi.org/10.1016/j.cmet.2011.03.006

Tsun, Z. Y., Bar-Peled, L., Chantranupong, L., Zoncu, R., Wang, T., Kim, C., ... Sabatini, D. M. (2013). The folliculin tumor suppressor is a GAP for the RagC/D GTPases that signal amino acid levels to mTORC1. Molecular Cell, 52(4), 495-505.

https://doi.org/10.1016/j.molcel.2013.09.016

Turgeon, M.-O., Perry, N. J. S., \& Poulogiannis, G. (2018). DNA Damage, Repair, and Cancer Metabolism. Frontiers in Oncology, 8. https://doi.org/10.3389/fonc.2018.00015

Valvezan, A. J., \& Manning, B. D. (2019). Molecular logic of mTORC1 signalling as a metabolic rheostat. Nature Metabolism 2019, 1. https://doi.org/10.1038/s42255-019-0038-7

Van Der Sluijs, P., Hull, M., Zahraoui, A., Tavitian, A., Goud, B., \& Mellman, I. (1991). The small GTP-binding protein rab4 is associated with early endosomes. Proceedings of the National Academy of Sciences of the United States of America, 88(14), 6313-6317. Retrieved from http://www.pubmedcentral.nih.gov/articlerender.fcgi?artid=52073\&tool=pmcentrez\&render type $=$ abstract

Vanhaesebroeck, B., Guillermet-Guibert, J., Graupera, M., \& Bilanges, B. (2010). The emerging mechanisms of isoform-specific PI3K signalling. Nature Reviews Molecular Cell Biology, 11(5), 329-341. https://doi.org/10.1038/nrm2882

Vanhaesebroeck, B., Stephens, L., \& Hawkins, P. (2012). PI3K signalling: the path to discovery and understanding. Nature Reviews Molecular Cell Biology, 13(3), 195-203. https://doi.org/10.1038/nrm3290

Vanlandingham, P. A., \& Ceresa, B. P. (2009). Rab7 regulates late endocytic trafficking downstream of multivesicular body biogenesis and cargo sequestration. Journal of Biological Chemistry, 284(18), 12110-12124. https://doi.org/10.1074/jbc.M809277200

Varki, A. (2017). Biological roles of glycans. Glycobiology, 27(1), 3-49. https://doi.org/10.1093/glycob/cww086

VÉZINA, C., KUDELSKI, A., \& SEHGAL, S. N. (1975). Rapamycin (AY-22,989), a new antifungal antibiotic. I. Taxonomy of the producing streptomycete and isolation of the active principle. The Journal of Antibiotics, 28(10), 721-726.

https://doi.org/10.7164/antibiotics.28.721 
Vinay, D. S., Keith, W. N., Ashraf, S. S., Nowsheen, S., Choi, B. K., Azmi, A. S., ... Aquilano, K. (2015). Immune evasion in cancer: Mechanistic basis and therapeutic strategies. Seminars in Cancer Biology, 35, S185-S198. https://doi.org/10.1016/j.semcancer.2015.03.004

Vinyoles, M., DelValle-Pérez, B., Curto, J., Viñas-Castells, R., Alba-Castellón, L., García de Herreros, A., \& Duñach, M. (2014). Multivesicular GSK3 Sequestration upon Wnt Signaling Is Controlled by p120-Catenin/Cadherin Interaction with LRP5/6. Molecular Cell, 53(3), 444-457. https://doi.org/10.1016/j.molcel.2013.12.010

Wagner, M. J., Stacey, M. M., Liu, B. A., \& Pawson, T. (2013). Molecular mechanisms of SH2and PTB-Domain-containing proteins in receptor tyrosine kinase signaling. Cold Spring Harbor Perspectives in Biology, 5(12). https://doi.org/10.1101/cshperspect.a008987

Waldmann, I., Wälde, S., \& Kehlenbach, R. H. (2007). Nuclear import of c-Jun is mediated by multiple transport receptors. Journal of Biological Chemistry, 282(38), 27685-27692. https://doi.org/10.1074/jbc.M703301200

Wang, L., Harris, T. E., Roth, R. A., \& Lawrence, J. C. (2007). PRAS40 regulates mTORC1 kinase activity by functioning as a direct inhibitor of substrate binding. Journal of Biological Chemistry, 282(27), 20036-20044. https://doi.org/10.1074/jbc.M702376200

Wang, S., Tsun, Z.-Y., Wolfson, R. L., Shen, K., Wyant, G. A., Plovanich, M. E., ... Sabatini, D. M. (2015). Lysosomal amino acid transporter SLC38A9 signals arginine sufficiency to mTORC1. Science, 347(6218), 188-194. https://doi.org/10.1126/science.1257132

Wang, T., Wolfson, S., Wyant, P., Yuan, J., Chantranupong, C., Wang, B.-P., ... Sabatini, S. (2015). Metabolism. Lysosomal amino acid transporter SLC38A9 signals arginine sufficiency to mTORC1. Science, 347(6218), 188-194.

Wang, Y., Shi, J., Chai, K., Ying, X., \& Zhou, B. P. (2013). The Role of Snail in EMT and Tumorigenesis. Current Cancer Drug Targets, 13(9), 963-972. Retrieved from http://www.ncbi.nlm.nih.gov/pubmed/24168186\%0Ahttp://www.pubmedcentral.nih.gov/art iclerender.fcgi?artid=PMC4004763

Warburg, O., Posener, K., \& Negelein, E. (1924). Ueber den Stoffwechsel der Tumoren. Biochemische Zeitschrift, 152, 309-344.

Warburg, O. (1956). On the Origin of Cancer Cells. Science, 123(3191), 309-314. https://doi.org/10.1126/science.123.3191.309

Warburg, O., Wind, F., \& Negelein, E. (1927). THE METABOLISM OF TUMORS IN THE BODY. The Journal of General Physiology, 8(6), 519-530.

https://doi.org/10.1085/jgp.8.6.519 
Ward, P. S., \& Thompson, C. B. (2012). Metabolic Reprogramming: A Cancer Hallmark Even Warburg Did Not Anticipate. Cancer Cell, 21(3), 297-308.

https://doi.org/10.1016/j.ccr.2012.02.014

Webb, J. L., Ravikumar, B., \& Rubinsztein, D. C. (2004). Microtubule disruption inhibits autophagosome-lysosome fusion: Implications for studying the roles of aggresomes in polyglutamine diseases. International Journal of Biochemistry and Cell Biology, 36(8), 1645-1654. https://doi.org/10.1016/j.biocel.2004.03.003

Wee, P., \& Wang, Z. (2017). Epidermal growth factor receptor cell proliferation signaling pathways. Cancers, 9(5). https://doi.org/10.3390/cancers9050052

Wei, W., \& Ji, S. (2018). Cellular senescence: Molecular mechanisms and pathogenicity. Journal of Cellular Physiology, 233(12), 9121-9135. https://doi.org/10.1002/jcp.26956

Welf, E. S., \& Haugh, J. M. (2011). Signaling pathways that control cell migration: models and analysis. Wiley Interdisciplinary Reviews: Systems Biology and Medicine, 3(2), 231-240. https://doi.org/10.1002/wsbm.110

Wente, S. R., \& Rout, M. P. (2010). The nuclear pore complex and nuclear transport. Cold Spring Harbor Perspectives in Biology, 2(10). https://doi.org/10.1101/cshperspect.a000562

Wiley, H. S. (1988). Anomalous binding of epidermal growth factor to A431 cells is due to the effect of high receptor densities and a saturable endocytic system. Journal of Cell Biology, 107(2), 801-810. https://doi.org/10.1083/jcb.107.2.801

Wilson, K. J., Gilmore, J. L., Foley, J., Lemmon, M. A., \& Riese, D. J. (2009). Functional selectivity of EGF family peptide growth factors: Implications for cancer. Pharmacology \& Therapeutics, 122(1), 1-8. https://doi.org/10.1016/j.pharmthera.2008.11.008

Wilson, K. J., Mill, C., Lambert, S., Buchman, J., Wilson, T. R., Hernandez-Gordillo, V., ... Riese, D. J. (2012). EGFR ligands exhibit functional differences in models of paracrine and autocrine signaling. Growth Factors, 30(2), 107-116. https://doi.org/10.3109/08977194.2011.649918

Witsch, E., Sela, M., \& Yarden, Y. (2010). Roles for Growth Factors in Cancer Progression. Physiology, 25(2), 85-101. https://doi.org/10.1152/physiol.00045.2009

Wolfer, A., \& Ramaswamy, S. (2011). MYC and metastasis. Cancer Research, 71(6), 20342037. https://doi.org/10.1158/0008-5472.CAN-10-3776

Wolfer, A., Wittner, B. S., Irimia, D., Flavin, R. J., Lupien, M., Gunawardane, R. N., ... Ramaswamy, S. (2010). MYC regulation of a "poor-prognosis" metastatic cancer cell state. Proceedings of the National Academy of Sciences, 107(8), 3698-3703. https://doi.org/10.1073/pnas.0914203107 
Wolfson, R. L., Chantranupong, L., Wyant, G. A., Gu, X., Orozco, J. M., Shen, K., ... Sabatini, D. M. (2017). KICSTOR recruits GATOR1 to the lysosome and is necessary for nutrients to regulate mTORC1. Nature, 543(7645), 438-442. https://doi.org/10.1038/nature21423

Woo, S.-Y., Kim, D.-H., Jun, C.-B., Kim, Y.-M., Haar, E. Vander, Lee, S., ... Kim, D.-H. (2007). PRR5, a Novel Component of mTOR Complex 2, Regulates Platelet-derived Growth Factor Receptor $\beta$ Expression and Signaling. Journal of Biological Chemistry, 282(35), 25604-25612. https://doi.org/10.1074/jbc.M704343200

Woodgett, J. R. (2018). Molecular cloning and expression of glycogen synthase kinase-3/factor A. The EMBO Journal, 9(8), 2431-2438. https://doi.org/10.1002/j.14602075.1990.tb07419.x

Wu, D., \& Pan, W. (2010). GSK3: a multifaceted kinase in Wnt signaling. Trends in Biochemical Sciences, 35(3), 161-168. https://doi.org/10.1016/j.tibs.2009.10.002

Xie, P. (2013). TRAF molecules in cell signaling and in human diseases. Journal of Molecular Signaling, 8, 7. https://doi.org/10.1186/1750-2187-8-7

Xu, X., Zur Hausen, A., Coy, J. F., \& Lochelt, M. (2009). Transketolase-like protein 1 (TKTL1) is required for rapid cell growth and full viability of human tumor cells. Int J Cancer, 124(6), 1330-1337. https://doi.org/10.1002/ijc.24078

Xu, Y., Lee, S. H., Kim, H. S., Kim, N. H., Piao, S., Park, S. H., ... Ha, N. C. (2010). Role of CK1 in GSK3B-mediated phosphorylation and degradation of Snail. Oncogene, 29(21), 3124-3133. https://doi.org/10.1038/onc.2010.77

Yadav, R. B., Burgos, P., Parker, A. W., Iadevaia, V., Proud, C. G., Allen, R. A., ... Botchway, S. W. (2013). mTOR direct interactions with Rheb-GTPase and raptor: Sub-cellular localization using fluorescence lifetime imaging. BMC Cell Biology, 14(1). https://doi.org/10.1186/1471-2121-14-3

Yamaguchi, H., Yoshida, S., Muroi, E., Yoshida, N., Kawamura, M., Kouchi, Z., ... Fukami, K. (2011). Phosphoinositide 3-kinase signaling pathway mediated by p110 $\alpha$ regulates invadopodia formation. Journal of Cell Biology, 193(7), 1275-1288. https://doi.org/10.1083/jcb.201009126

Yamaguchi, M., Noda, N. N., Yamamoto, H., Shima, T., Kumeta, H., Kobashigawa, Y., ... Inagaki, F. (2012). Structural insights into Atg10-mediated formation of the autophagyessential Atg12-Atg5 conjugate. Structure, 20(7), 1244-1254. https://doi.org/10.1016/j.str.2012.04.018

Yang, A., Qin, S., Schulte, B. A., Ethier, S. P., Tew, K. D., \& Wang, G. Y. (2017). MYC inhibition depletes cancer stem-like cells in triple negative breast cancer. Cancer Research, 77(23), 6641-6650. https://doi.org/10.1158/0008-5472.CAN-16-3452 
Yang, H., Rudge, D. G., Koos, J. D., Vaidialingam, B., Yang, H. J., \& Pavletich, N. P. (2013). MTOR kinase structure, mechanism and regulation. Nature, 497(7448), 217-223. https://doi.org/10.1038/nature12122

Yang, Q., Inoki, K., Ikenoue, T., \& Guan, K.-L. (2006). Identification of Sin1 as an essential TORC2 component required for complex formation and kinase activity. Genes \& Development, 20(20), 2820-2832. https://doi.org/10.1101/gad.1461206

Yang, Y.-P., Ma, H., Starchenko, A., Huh, W. J., Li, W., Hickman, F. E., ... Coffey, R. J. (2017). A Chimeric Egfr Protein Reporter Mouse Reveals Egfr Localization and Trafficking In Vivo. Cell Reports, 19(6), 1257-1267. https://doi.org/10.1016/j.celrep.2017.04.048

Yarden, Y., \& Pines, G. (2012). The ERBB network: at last, cancer therapy meets systems biology. Nature Reviews Cancer, 12(8), 553-563. https://doi.org/10.1038/nrc3309

Ying, H., Kimmelman, A. C., Lyssiotis, C. A., Hua, S., Chu, G. C., Fletcher-Sananikone, E., ... Depinho, R. A. (2012). Oncogenic kras maintains pancreatic tumors through regulation of anabolic glucose metabolism. Cell, 149(3), 656-670.

https://doi.org/10.1016/j.cell.2012.01.058

Yong, H.-Y., Hwang, J.-S., Son, H., Park, H.-I., Oh, E.-S., Kim, H.-H., .. Moon, A. (2011). Identification of H-Ras-Specific Motif for the Activation of Invasive Signaling Program in Human Breast Epithelial Cells. Neoplasia, 13(2), 98-IN1. https://doi.org/10.1593/neo.101088

Yoshida, G. J. (2015). Metabolic reprogramming: The emerging concept and associated therapeutic strategies. Journal of Experimental and Clinical Cancer Research, 34(1). https://doi.org/10.1186/s13046-015-0221-y

Yu, L., Chen, Y., \& Tooze, S. A. (2018). Autophagy pathway: Cellular and molecular mechanisms. Autophagy, 14(2), 207-215. https://doi.org/10.1080/15548627.2017.1378838

Yun, C. L., \& Zierath, J. R. (2006). AMP-activated protein kinase signaling in metabolic regulation. Journal of Clinical Investigation, 116(7), 1776-1783. https://doi.org/10.1172/JCI29044

Zachary E. Stine, Zandra E. Walton, Brian J. Altman, Annie L. Hsieh, and C. V. D. (2015). MYC, Metabolism, and Cancer. Cancer Discov., 5(10), 1024-1039. https://doi.org/10.1038/nbt.3121.ChIP-nexus

Zeng, X., Huang, H., Tamai, K., Zhang, X., Harada, Y., Yokota, C., .. He, X. (2007). Initiation of Wnt signaling: control of Wnt coreceptor Lrp6 phosphorylation/activation via frizzled, dishevelled and axin functions. Development, 135(2), 367-375. https://doi.org/10.1242/dev.013540 
Zeng, X., Tamai, K., Doble, B., Li, S., Huang, H., Habas, R., ... He, X. (2005). A dual-kinase mechanism for Wnt co-receptor phosphorylation and activation. Nature, 438(7069), 873877. https://doi.org/10.1038/nature04185

Zerial, M., Christoforidis, S., McBride, H. M., \& Burgoyne, R. D. (1999). The Rab5 effector EEA1 is a core component of endosome docking. Nature, 397(6720), 621-625. https://doi.org/10.1038/17618

Zhang W, L. H. (2002). MAPK signal pathways in the regulation of cell proliferation in mammalian cells. Cell Res., 12, 9-18. https://doi.org/10.1007/BF00568631

Zhang, X., Tang, N., Hadden, T. J., \& Rishi, A. K. (2011). Akt, FoxO and regulation of apoptosis. Biochimica et Biophysica Acta - Molecular Cell Research, 1813(11), 1978-1986. https://doi.org/10.1016/j.bbamcr.2011.03.010

Zhang, Y., Huang, N. qu, Yan, F., Jin, H., Zhou, S. yu, Shi, J. shan, \& Jin, F. (2018). Diabetes mellitus and Alzheimer's disease: GSK-3 $\beta$ as a potential link. Behavioural Brain Research, 339, 57-65. https://doi.org/10.1016/j.bbr.2017.11.015

Zhou, B. P., Deng, J., Xia, W., Xu, J., Li, Y. M., Gunduz, M., \& Hung, M. C. (2004). Dual regulation of Snail by GSK-3 $\beta$-mediated phosphorylation in control of epithelialmesenchymal transition. Nature Cell Biology, 6(10), 931-940.

https://oi.org/10.1038/ncb1173

Zhou, X., Clister, T. L., Lowry, P. R., Seldin, M. M., Wong, G. W., \& Zhang, J. (2015). Dynamic Visualization of mTORC1 Activity in Living Cells. Cell Reports, 10(10), 17671777. https://doi.org/10.1016/j.celrep.2015.02.031

Zhu, J., Blenis, J., \& Yuan, J. (2008). Activation of PI3K/Akt and MAPK pathways regulates Myc-mediated transcription by phosphorylating and promoting the degradation of Mad1. Proceedings of the National Academy of Sciences, 105(18), 6584-6589. https://doi.org/10.1073/pnas.0802785105

Zihni, C., Mills, C., Matter, K., \& Balda, M. S. (2016). Tight junctions: From simple barriers to multifunctional molecular gates. Nature Reviews Molecular Cell Biology, 17(9), 564-580. https://doi.org/10.1038/nrm.2016.80

Zmijewski, J. W., \& Jope, R. S. (2004). Nuclear accumulation of glycogen synthase kinase-3 during replicative senescence of human fibroblasts. Aging Cell, 3(5), 309-317. https://doi.org/10.1111/j.1474-9728.2004.00117.x

Zoncu, R., Bar-Peled, L., Efeyan, A., Wang, S., Sancak, Y., \& Sabatini, D. M. (2011). mTORC1 Senses Lysosomal Amino Acids Through an Inside-Out Mechanism That Requires the Vacuolar H+-ATPase. Science, 334(6056), 678-683. https://doi.org/10.1126/science.1207056 
Zoncu, R., Perera, R. M., Balkin, D. M., Pirruccello, M., Toomre, D., \& De Camilli, P. (2009). A Phosphoinositide Switch Controls the Maturation and Signaling Properties of APPL

Endosomes. Cell, 136(6), 1110-1121. https://doi.org/10.1016/j.cell.2009.01.032 